\title{
BPS black hole entropy and attractors in very special geometry. Cubic forms, gradient maps and their inversion
}

\author{
Bert van Geemen, ${ }^{a}$ Alessio Marrani ${ }^{b, c}$ and Francesco Russo ${ }^{d}$ \\ ${ }^{a}$ Dipartimento di Matematica, Università di Milano, \\ Via Saldini 50, I-20133 Milano, Italy \\ ${ }^{b}$ Centro Studi e Ricerche Enrico Fermi, \\ Via Panisperna 89A, I-00184, Roma, Italy \\ ${ }^{c}$ Dipartimento di Fisica e Astronomia Galileo Galilei, Università di Padova, \\ and INFN, sezione di Padova, Via Marzolo 8, I-35131 Padova, Italy \\ ${ }^{d}$ Dipartimento di Matematica e Informatica, Università di Catania, \\ Viale A. Doria 5, I-95125 Catania, Italy \\ E-mail: lambertus.vangeemen@unimi.it, jazzphyzz@gmail.com, \\ frusso@dmi.unict.it
}

ABSTRACT: We consider Bekenstein-Hawking entropy and attractors in extremal BPS black holes of $\mathcal{N}=2, D=4$ ungauged supergravity obtained as reduction of minimal, matter-coupled $D=5$ supergravity. They are generally expressed in terms of solutions to an inhomogeneous system of coupled quadratic equations, named BPS system, depending on the cubic prepotential as well as on the electric-magnetic fluxes in the extremal black hole background. Focussing on homogeneous non-symmetric scalar manifolds (whose classification is known in terms of $L(q, P, \dot{P})$ models), under certain assumptions on the Clifford matrices pertaining to the related cubic prepotential, we formulate and prove an invertibility condition for the gradient map of the corresponding cubic form (to have a birational inverse map which is given by homogeneous polynomials of degree four), and therefore for the solutions to the BPS system to be explicitly determined, in turn providing novel, explicit expressions for the BPS black hole entropy and the related attractors as solution of the BPS attractor equations. After a general treatment, we present a number of explicit examples with $\dot{P}=0$, such as $L(q, P), 1 \leqslant q \leqslant 3$ and $P \geqslant 1$, or $L(q, 1), 4 \leqslant q \leqslant 9$, and one model with $\dot{P}=1$, namely $L(4,1,1)$. We also briefly comment on Kleinian signatures and split algebras. In particular, we provide, for the first time, the explicit form of the BPS black hole entropy and of the related BPS attractors for the infinite class of $L(1, P) P \geqslant 2$ non-symmetric models of $\mathcal{N}=2, D=4$ supergravity.

KEYwords: Black Holes in String Theory, Supergravity Models, Differential and Algebraic Geometry

ArXIV EPrINT: 2009.10647 


\section{Contents}

1 Introduction 1

2 BPS black hole entropy and attractors in very special geometry 5

3 BPS systems and the gradient map $\nabla_{\mathcal{V}} \quad 8$

4 Homogeneous very special geometry $\quad 9$

4.1 Symmetric $d$-manifolds $\quad 9$

4.2 Non-symmetric $d$-manifolds $\quad 11$

4.3 Classification of homogeneous $d$-manifolds: $L(q, P, \dot{P}) \quad 12$

4.3.1 $L(q, 1)$ and "magic" enhancements 16

5 Basics on Euclidean Clifford algebras $\quad 19$

$\begin{array}{lll}5.1 & \text { Euclidean Clifford algebras } & 19\end{array}$

5.2 Representations of $C l(n, 0)$ and Clifford sets 20

5.3 The product decomposition for $n \equiv 1 \bmod 4 \quad 21$

5.4 Quadratic forms and $\Gamma$-matrices 22

5.5 Examples of the quadratic forms 23

5.6 Clifford sets of $\Gamma$-matrices 23

5.7 Quadratic identities between quadratic forms 24

5.8 Heisenberg groups 24

6 BPS entropy and attractors in $L(q, P)$ models $\quad 24$

6.1 The cubic form 26

$\begin{array}{ll}6.2 & \text { The invertibility condition } \\ 6.3 & \text { Remarks }\end{array}$

$\begin{array}{lll}6.3 & \text { Remarks } & 27\end{array}$

6.4 Explicit inversion of $\nabla \mathcal{V} \quad 27$

6.5 Solution of the BPS system 30

6.5.1 The symmetric case 34

6.6 BPS black hole entropy and attractors 34

6.7 Complete models: $r=0 \quad 36$

$\begin{array}{lll}7 & \text { Verifying the inverse map } & 37\end{array}$

7.1 Factorization of $R_{K}$ 's

$\begin{array}{ll}7.2 \text { The map } \alpha \circ \nabla \mathcal{V} & 38\end{array}$

$\begin{array}{lll}7.3 & \text { The map } \mu \circ \alpha \circ \nabla_{\mathcal{V}} & 38\end{array}$

8 Examples, I: $L(q, 1), q=1, \ldots, 8 \quad 43$

$8.1 L(8,1) \equiv J_{3}^{\mathbb{Q}} \quad 43$

8.2 Some submodels of $L(8,1) \quad 45$

$\begin{array}{lll}8.2 .1 & L(7,1) & 45\end{array}$

$\begin{array}{lll}8.2 .2 & L(6,1) & 46\end{array}$ 
$\begin{array}{lll}8.2 .3 & L(5,1) & 47\end{array}$

$\begin{array}{ll}8.2 .4 L(4,1) \equiv J_{3}^{\mathbb{H}} & 48\end{array}$

$\begin{array}{lll}8.2 .5 & L(3,1) & 50\end{array}$

$\begin{array}{ll}8.2 .6 & L(2,1) \equiv J_{3}^{\mathbb{C}}\end{array}$

$\begin{array}{ll}8.2 .7 & L(1,1) \equiv J_{3}^{\mathbb{R}}\end{array} 52$

9 Examples, II: $L(q, 2), q=1,2,3$

$\begin{array}{lll}9.1 & \text { Block decompositions } & 55\end{array}$

$\begin{array}{ll}9.2 P=2 & 55\end{array}$

$\begin{array}{lll}9.3 & L(3,2) & 56\end{array}$

$\begin{array}{lll}9.4 & L(2,2) & 57\end{array}$

$\begin{array}{lll}9.5 & L(1,2) & 59\end{array}$

10 Examples, III: $L(q, P), q=1,2,3, P \geqslant 3$

$\begin{array}{ll}10.1 \text { Block }(k, l) \text {-lifts } & 60\end{array}$

$\begin{array}{ll}10.2 & \text { Invertibility } \\ \end{array}$

$\begin{array}{ll}10.3 \text { Proof of the Lorentzian identity (10.4) } & 61\end{array}$

$\begin{array}{ll}10.4 L(3,3) & 63\end{array}$

$10.5 L(2,3) \quad 64$

$\begin{array}{lll}10.6 & L(1,3) & 65\end{array}$

$\begin{array}{ll}10.7 L(1, P) & 65\end{array}$

10.7.1 $P \geqslant 2 \quad 65$

10.7.2 On the geometry of $\left(\nabla_{\mathcal{V}_{L(1, P)}}\right)^{-1}, P \geqslant 1 \quad 70$

11 Non-uniqueness of $\Omega$ 's $\quad 71$

$\begin{array}{ll}11.1 L(1,4) \subset L(4,1) & 71\end{array}$

$\begin{array}{ll}11.2 L(1,8) \subset L(8,1) & 72\end{array}$

$\begin{array}{ll}11.3 \text { Descendant } \nrightarrow \text { submodel } & 73\end{array}$

12 Beyond $L(q, P)$ models: $L(4,1,1) \quad 75$

$\begin{array}{ll}13 \text { Kleinian signatures and split algebras } & 77\end{array}$

14 Beyond the invertibility condition: $L(9,1) \quad 78$

$\begin{array}{lr}15 \text { Final remarks and outlook } & 79\end{array}$

$\begin{array}{lr}\text { A Details on } L(1,2) & 81\end{array}$

$\begin{array}{ll}\text { B Details on } L(1,3) & 85\end{array}$

$\begin{array}{lll}\text { C A calculation in Lie theory } & 89\end{array}$ 


\section{Introduction}

In the last decades, the theoretical and phenomenological implications of the physics of black holes [73-75] had a profound and fertile impact on many branches of science, from astrophysics, cosmology, particle physics, to mathematical physics [100], quantum information theory [48-50], and, recently, number theory [15]. Remarkably, the singularity theorems proved by Penrose and Hawking [84] imply that the black holes are an unavoidable consequence of Einstein's theory of General Relativity, as well as of its modern generalizations such as supergravity [20-22, 44-46, 120, 121], superstrings and M-theory [23-28]. Classically, the gravitational force inside the event horizon of a black hole is so strong that nothing, not even light, can escape. However, in the 70s Hawking showed that quantum effects cause black holes to thermally radiate, and eventually evaporate [81].

While the frontiers of physics are progressing also the 21st century, it should not be forgotten that the physics of the 20th century is conceptually founded on two theories which are mutually incompatible. On one side, Quantum Mechanics governs the microscopic world of the basic constituents of matter, such as molecules, atoms, nuclei and beyond. On the other side, General Relativity describes gravity and the macroscopic, large-scale structures, ranging from planetary orbits to the Universe in its entirety. As the energy increases, Quantum Mechanics and General Relativity inevitably meet, giving rise to startling, even paradoxical, consequences.

A tantalizing aspect of the physics of a black hole is that its thermodynamical features seem to encode fundamental insights of a not yet formulated theory of Quantum Gravity, which should necessarily arise from the reconciliation of the two aforementioned apparently contradictory physical theories. In this framework, a crucial relevance owes to the BekensteinHawking entropy-area formula [82, 83]:

$$
S=\frac{k_{B}}{\ell_{P}^{2}} \frac{A_{H}}{4},
$$

where $^{1} k_{B}$ is the Boltzmann constant, $\ell_{P}^{2}=G \hbar / c^{3}$ is the squared Planck length, whereas $A_{H}$ denotes the area of the event horizon of the black hole itself. This formula relates a thermodynamical quantity (the entropy $S$ ) to a geometric quantity (the area $A_{H}$ ), and after much theoretical work it still puzzles the scientific community. In fact, a crucial issue that Quantum Gravity must necessarily address concerns on the origin of $S$ at a fundamental level. At the classical level, (1.1) yields that black hole entropy is determined by the area of the event horizon, which is a macroscopic and geometric quantity; however, black hole entropy must also enjoy a microscopic, statistical derivation, accounting for fundamental microscopic degrees of freedom.

Since superstring theory and M-theory are the most serious candidates for a theory of Quantum Gravity, they are expected to provide a microscopic, statistical explanation of the entropy-area law (1.1) [92-97]. Black holes are typical non-perturbative objects, since they describe a physical regime in which the gravitational field is very strong; thus, only a non-perturbative approach can successfully deal with them. Progress in this direction

\footnotetext{
${ }^{1}$ We will use the so-called natural units henceforth: $\hbar=c=G=k_{B}=1$.
} 
came after 1995 [138], through the recognition of the role of string dualities, which allow one to relate the strong coupling regime of one superstring model to the weak coupling regime of another. Remarkably, there is evidence that the string dualities are all encoded into the global symmetry group (the $U$-duality, also named electric-magnetic, group) of the low energy supergravity effective action [85].

Black holes, and in particular their extremal configurations [57, 58], are embedded in a natural way in supergravity theories, which, being invariant under local super-Poincaré transformations, include General Relativity, providing a consistent description of the graviton coupled to other fields in a supersymmetric framework. Extremal black holes have become objects of crucial relevance in the context of superstrings after 1995 [20-28, 92$97,111,112]$ : the classical solutions of supergravity that preserve a fraction of the original supersymmetries can be interpreted as non-perturbative states, necessary to complete the perturbative string spectrum and make it invariant under the many conjectured duality symmetries $[68,69,85,118,119,130-132,136,137]$. In such a framework, extremal black holes, as well as their parent $p$-branes in higher dimensions, are conceived as additional particle-like states that compose the spectrum of a fundamental quantum theory. Similar to monopoles in gauge theories, these non-perturbative quantum states originate from regular solutions of the classical field equations, i.e. the very same Einstein equations on which General Relativity relies. The crucial new ingredient, in this respect, is Supersymmetry, which requires a precise balance between vector fields and scalar fields in the bosonic spectrum of the theory. As such, the general framework we are going to deal with is provided by the so-called Einstein-Maxwell-scalar theories, whose global mathematical thorough treatment has been recently given in [91] (see also [12]).

Supergravity theories provide a low-energy effective theory description of superstring and M-theory, holding at the lowest order in the string loop expansion, when the space-time curvature is much smaller than the typical string scale (string tension). Consequently, the supergravity description of extremal black holes can be trusted only when the radius of the event horizon is much larger than the string scale, corresponding to the regime of large charges. We will not be dealing with further corrections, introduced by string theory, which give rise to higher derivative terms in the low energy effective action, such that the black hole entropy is expected to be corrected by subleading terms in the limit of small curvature:it is well known that these corrections determine a deviation from the area law for the entropy [35, 36, 139].

The cosmic censorship conjecture [88] is naturally realized by conceiving extremal black holes as solitonic solutions of $\mathcal{N}$-extended locally supersymmetric theory of Einstein gravity: in fact, denoting with $\mathcal{N}$ the number of spinor supercharges in $3+1$ space-time dimensions, when $\mathcal{N} \geqslant 2$ the so-called BPS (Bogomol'nyi-Prasad-Sommerfeld) bound [32, 33],

$$
M \geqslant|Q|,
$$

where $M$ and $Q$ respectively are the mass and the magnetic (or electric) charge of the black hole, is just a consequence of the supersymmetry algebra, implying that no naked singularities can occur. 
When the black hole solution is embedded into a $\mathcal{N}$-extended supergravity theory, the model is characterized by a certain $\mathcal{N}$-dependent number of scalar fields, collectively denoted by $\phi$. In this framework, the charge $Q$ is to be replaced by the maximum eigenvalue of the $\mathcal{N} \times \mathcal{N}$ central charge matrix appearing in the r.h.s. of the supersymmetry algebra (depending on the expectation value $\phi_{H}(p, q)$ of the scalar fields on the event horizon, where $p$ 's and $q$ 's respectively denote magnetic and electric charges of the black hole):

$$
M=M(p, q) \geqslant\left|Z_{\max }\left(\phi_{H}(p, q), p, q\right)\right|
$$

In the present paper, we will be dealing only with extremal black holes, in which the BPS bound (1.2)-(1.3) is saturated.

Extremal black holes enjoy the following peculiar and crucial feature: despite the fact that the dynamics depends on scalar fields, the event horizon of the black hole loses all information about the scalars, and this holds regardless of the supersymmetrypreserving features of the solution. This phenomenon is described by the so-called attractor mechanism [8-11, 18]: independently of their boundary conditions at spatial infinity, scalar fields flow to a fixed point given by a certain ratio of electric and magnetic charges, when approaching the event horizon. In this framework, the scalar fields are moduli, i.e. they are continuous parameters which can be freely specified at infinity, raising the dangerous possibility that the black hole entropy might depend on their values. Indeed, such a dependence presumably would lead to a violation of the second law of thermodynamics, since it would allow one to quasi-statically decrease the entropy by varying the moduli. Instead, the black hole entropy turns out to depend only on the values acquired by the scalar fields at the event horizon, which in turn only depend on the conserved charges $(p$ and $q$ ) associated to gauge invariance of the black hole solution itself: in this sense, the entropy of extremal black holes is a topological quantity, because it is fixed in terms of the quantized electric and magnetic charges, while it does not depend on moduli.

For extremal black holes in Maxwell-Einstein supergravity theories with $\mathcal{N}=2$ local supersymmetry in $3+1$ space-time dimensions [5], the saturation of the BPS bound (1.3),

$$
M(p, q)=\left|Z\left(\phi_{H}(p, q), p, q\right)\right|,
$$

yields, for the black hole entropy,

$$
S(p, q)=\frac{A_{H}(p, q)}{4}=\pi\left|Z\left(\phi_{H}(p, q), p, q\right)\right|^{2},
$$

where $Z$ is the $\mathcal{N}=2$ central charge function [34]. The attractor values of the scalar fields at the event horizon, here collectively denoted by $\phi_{H}(p, q)$, arise as solutions to the so-called BPS attractor equations:

$$
\left.\mathcal{D}_{\phi} Z(\phi, p, q)\right|_{\phi=\phi_{H}(p, q)}=0,
$$

where $\mathcal{D}_{\phi}$ denotes the Kähler-covariant differential operator acting on the scalar manifold (target space of moduli fields). The entropy $S$ generally enjoys a $U$-duality-invariant expression (homogeneous of degree two) in terms of electric and magnetic charges, only 
depending on the nature of the $U$-duality groups and on the appropriate representations of electric and magnetic charges [3].

Through the years, the attractor mechanism has been discovered to have a broader application [16, 17, 61, 105-109, 113, 114] beyond the BPS cases, being a peculiarity of all extremal black-holes, BPS or not. Even for these more general cases, because of the topological nature of the extremality condition, the entropy formula turns out to be still given by a $U$-duality invariant expression built out of electric and magnetic charges.

The present paper is devoted to the determination of the explicit expression of two purely charge-dependent quantities, characterizing the physics of BPS extremal black holes: the Bekenstein-Hawking entropy $S(p, q)$ and the attractor values, collectively denoted by $\phi_{H}(p, q)$, acquired by the scalar fields when approaching the (unique) event horizon (regardless of the boundary conditions of their evolution dynamics). We will consider ungauged Maxwell-Einstein supergravity theories with $\mathcal{N}=2$ extended local supersymmetry in $3+1$ space-time dimensions, in the case in which the special Kähler geometry of the vector multiplets' scalar manifold is determined by a cubic holomorphic prepotential (very special geometry). In fact, in such a framework only the models in which the scalar manifold is a symmetric coset have been thoroughly investigated: exploiting the relation to the theory of cubic (simple and semisimple) Jordan algebras and related Freudenthal triple systems, which hold in all cases but the so-called Luciani models (with quadratic prepotential), the explicit expressions of $S(p, q)$ and of $\phi_{H}(p, q)$ have been explicitly computed for extremal, both BPS and non-BPS, black holes (cfr. e.g. [62] and refs. therein).

On the other hand, very little is known in the case in which the (vector multiplets') scalar manifold is not symmetric. In very special geometry, the BPS entropy and the BPS attractor values of the scalar fields have been computed by Shmakova [122], up to the solution of an inhomogeneous system of quadratic algebraic equations, named BPS system. A noteworthy, countably infinite class of cubic non-symmetric models is provided by homogeneous non-symmetric models, which have been classified in [53] (in a mathematical context, see also the subsequent classification in [43]). These models are quite interesting from a physical point of view, because some of them naturally occur in the four dimensional effective supergravity description of brane dynamics, when their brane and bulk degrees of freedom get unified. To the best of our knowledge, only [51] dealt with such a class of models, but did not investigate the explicit determination of $S(p, q)$ and $\phi_{H}(p, q)$. In the present work, we will rely on the existing classification of homogeneous non-symmetric special manifolds, and we will formulate a (sufficient but not necessary) condition for the BPS system to be explicitly solved. Thus, within the validity of such a condition (which we will prove to actually hold for an infinite, countable number of models), we will explicitly determine the expression of the Bekenstein-Hawking (semi)classical black hole entropy $S(p, q)$ as well as of the purely charge-dependent attractor values $\phi_{H}(p, q)$ acquired by the scalar fields at the event horizon of asymptotically flat, spherically symmetric, static, dyonic, extremal BPS black holes.

The paper is organized as follows.

In sections 2-3 we introduce the Bekenstein-Hawking BPS black hole entropy and the BPS attractor values of scalar fields in $\mathcal{N}=2$ very special geometry, relating their 
explicit expressions to the solution of the corresponding BPS system, or equivalently to the inversion of the gradient map of the cubic form defining the corresponding cubic holomorphic prepotential. Then, in section 4 we specialize the treatment to homogeneous very special geometry, briefly recalling some basic facts on symmetric and non-symmetric spaces in sections 4.1 resp. 4.2. In section 4.3 we review the classification of homogeneous special $d$-spaces [53], and in section 4.3 .1 we briefly consider the class $L(q, 1)$. Section 5 , which is in turn split into eight subsections, is then devoted to the introduction and review of another important ingredient of our treatment: Euclidean Clifford algebras. Next, sections 6 and 7 contain the main results of the present paper: after enouncing an invertibility condition for the gradient map of the cubic form in section 6.2 (then proved in section 7), the BPS system is explicitly solved in sections 6.4-6.5, and the explicit expressions of the BPS entropy and of the BPS attractor values of scalar fields at the horizon are computed in section 6.6. Then, section 6.7 introduces the so-called complete models, whose known examples coincide with the symmetric $d$-spaces, recalled in section 6.5.1. Sections 8, 9 and 10 present a threefold wealth of models in which the invertibility condition of section 6.2 holds true, and in some cases, such as the models $L(1,2)$ and $L(1,3)$ in sections 9.5 resp. 10.6 (then generalized $^{2}$ as $L(1, P)$ with $P \geqslant 2$ in section 10.7$)$, the corresponding BPS system is explicitly solved (with details given in appendices $\mathrm{A}$ and $\mathrm{B}$ ), thus providing the fully fledged expressions of the BPS entropy and attractors. The non-uniqueness of the matrices $\Omega_{K}$ 's occurring in the invertibility condition of section 6.2 is discussed in section 11, and in section 11.3 the difference between descendant models and submodels is highlighted (with details given in appendix C). Moreover, section 12 discusses the unique model of the present paper which has a non-vanishing $\dot{P}$ : namely, the model $L(4,1,1)$. A brief discussion of cubic models determined by Kleinian (rather than Lorentzian) quadratic polynomials (thus giving rise to a non-special geometry) is provided in section 13. Finally, section 14 deals with the model $L(9,1)$, as an example of model in which the invertibility criterion of section 6.2 does not seem to be applicable, and thus other approaches are needed to prove or disprove the invertibility of the BPS system. Finally, some outlook and hints for further developments are provided in the concluding section 15 .

\section{BPS black hole entropy and attractors in very special geometry}

A large class of four-dimensional Maxwell-Einstein gravity theories with local $\mathcal{N}=2$ supersymmetry can be obtained by an $S^{1}$-compactification of five-dimensional minimal supergravity. In such a case, the Kähler-Hodge geometry of the vector multiplets' scalar manifolds in $D=4$ is named very special [1, 64, 123-129, 133], and it is determined by an holomorphic prepotential of the type ${ }^{3}$

$$
F(X):=\frac{1}{3 !} d_{i j k} \frac{X^{i} X^{j} X^{k}}{X^{0}}
$$

\footnotetext{
${ }^{2}$ In section 10.7.2 we will also briefly present a geometric point of view on the factorization of the inverse map of the gradient map, whose detailed investigation goes beyond the scope of this paper.

${ }^{3}$ Einstein summation convention on repeated indices is understood throughout. Lowercase Latin indices run $1, \ldots, N$ throughout; $N$ denotes the number of vector multiplets. The index 0 pertains to the $(D=4)$ graviphotonic sector.
} 
where $d_{i j k}$ is a completely symmetric real tensor, and the $X^{\Lambda}$ 's, $\Lambda=0, i$, are the contravariant symplectic sections of the Kähler-Hodge target space of scalar fields. The symplectic frame in which $F(X)(2.1)$ is specified is the one of the so-called " $4 D / 5 D$ special coordinates" (see for instance $[1,39]$ ): the manifest symmetry is the electric-magnetic $(U-)$ duality ${ }^{4}$ group of the parent theory in $D=5$ (which leaves $d_{i j k}$ invariant).

From the treatment given in [122], in the general case in which all electric and magnetic charges associated to the black hole solution are non-vanishing, the Bekenstein-Hawking entropy of static, spherically symmetric, BPS extremal dyonic black holes in ungauged $\mathcal{N}=2, D=4$ Maxwell-Einstein supergravity whose vector multiplets' scalar manifold displays a very special Kähler geometry, reads ${ }^{5}$

$$
\frac{S}{\pi}=\frac{1}{3\left|p^{0}\right|} \sqrt{\frac{4}{3}\left(\Delta_{i} x^{i}\right)^{2}-9\left[p^{0}(p \cdot q)-2 I_{3}(p)\right]^{2}},
$$

where $p^{0}, p^{i}, q_{0}$ and $q_{i}$ are the magnetic resp. electric black hole charges, and

$$
\begin{aligned}
\Delta_{i} & :=\frac{1}{2} d_{i j k} p^{j} p^{k}-p^{0} q_{i}=\frac{\partial I_{3}(p)}{\partial p^{i}}-p^{0} q_{i} ; \\
p \cdot q & :=p^{0} q_{0}+p^{i} q_{i} ; \\
I_{3}(p) & :=\frac{1}{3 !} d_{i j k} p^{i} p^{j} p^{k} .
\end{aligned}
$$

Note that, as it must be, $S(2.2)$ is homogeneous of degree 2 in the black hole charges. Furthermore, $q_{0}$ enters the expression (2.2) only through the quantity $p \cdot q(2.4)$.

The $x^{i}$ s appearing in $(2.2)$ are the solutions $x^{i}\left(\Delta_{j} ; d_{k l m}\right)$ of the system of algebraic quadratic equations

$$
\frac{1}{3 !} d_{i j k} x^{j} x^{k}=\Delta_{i}
$$

which we will henceforth name the BPS system ${ }^{6}$ pertaining to the model of $\mathcal{N}=2, D=4$ ungauged supergravity (coupled to vector multiplets ${ }^{7}$ ) under consideration. Since the $\Delta_{i}$ 's are homogeneous of degree two in the black hole charges $p^{i}, p^{0}$ and $q_{i},(2.6)$ implies that the $x^{i}$ s are homogeneous of degree one in the same variables:

$$
\frac{\partial x^{i}}{\partial p^{j}} p^{j}+\frac{\partial x^{i}}{\partial p^{0}} p^{0}+\frac{\partial x^{i}}{\partial q_{j}} q_{j}=x^{i}
$$

Furthermore, the $x^{i}$ 's contribute to the black hole entropy only through the square of the quantity $^{8}$

$$
\Delta_{i} x^{i}=\frac{1}{3 !} d_{i j k} x^{i} x^{j} x^{k}=: \mathcal{V}(x) .
$$

\footnotetext{
${ }^{4}$ In this paper, $U$-duality is referred to as the "continuous" symmetries of [41, 42]. Their discrete versions are the $U$-duality non-perturbative string theory symmetries introduced by Hull and Townsend [85].

${ }^{5}$ This formula fixes a typo in eq. (12) of [122]. For further, recent insight on the BPS entropy in very special geometry, see [12].

${ }^{6}$ Let us recall that the explicit solution of the BPS system is also relevant for the solution of the attractor equations in asymptotically AdS, dyonic, extremal $\frac{1}{4}$-BPS black holes of U(1) Fayet-Iliopoulos gauged Maxwell-Einstein $\mathcal{N}=2$ supergravity in four space-time dimensions [65, 79].

${ }^{7}$ The coupling to hypermultiplets can be disregarded, because their equations of motion decouple completely in the ungauged case, and so they do not contribute at all to the extremal black hole entropy.

${ }^{8}$ Recalling (2.5), such a definition implies that $I_{3}(p)=\mathcal{V}(p)$.
} 
The number of equations in the system (2.6) is equal to the number $N$ of complex scalar fields, which in the large volume limit of Calabi-Yau compactifications of type II superstrings correspond to the Calabi-Yau moduli fields. The l.h.s. of (2.6) is given by quadratic forms with coefficients $d_{(i) j k}$, while the r.h.s. is arbitrary and depends on the values of electric and magnetic charges of the extremal BPS black hole. In other words, it defines $N$ quadratic hypersurfaces (each of dimension $N-1$ ) in an $N$-dimensional space, and the intersection of these hypersurfaces is the set of solutions of the system (2.6). Therefore, the BPS system may also not admit any analytical (or in closed form) real solution at all.

Note that the condition

$$
\frac{4}{3}\left(\Delta_{i} x^{i}\right)^{2}-9\left[p^{0}(p \cdot q)-2 I_{3}(p)\right]^{2}>0
$$

is a consistency condition for the BPS entropy (2.2) to be well defined. We will see below what is the general (set of) BPS condition(s) in $\mathcal{N}=2$ ungauged supergravity with cubic prepotential (cfr. (2.14) below).

By switching the notation of scalar fields (at the horizon) from $\phi_{H}$ to $z_{H}^{i}$, and denoting with $\mathbf{i}$ the imaginary unit of $\mathbb{C}$, the explicit solutions to the BPS Attractor Equations read $[122]$

$$
\begin{aligned}
z_{H}^{i}\left(p^{0}, p^{k}, q_{0}, q_{k}\right) & =\frac{3}{2} \frac{x^{i}}{p^{0} \Delta_{j} x^{j}}\left[p^{0}(p \cdot q)-2 I_{3}(p)\right]+\frac{p^{i}}{p^{0}}-\mathbf{i} \frac{3}{2} \frac{x^{i}}{\left|\Delta_{j} x^{j}\right|} \frac{S}{\pi} \\
& =\frac{3}{2} \frac{x^{i}}{\Delta_{j} x^{j}}\left[\frac{p^{0}(p \cdot q)-2 I_{3}(p)}{p^{0}}-\mathbf{i} \frac{S}{\pi} \operatorname{sgn}\left(\Delta_{j} x^{j}\right)\right]+\frac{p^{i}}{p^{0}},
\end{aligned}
$$

where $S$ is given by (2.2), and the subscript " $H$ " denotes the evaluation at the (unique) event horizon of the extremal BPS black hole.

Finally, it is here worth recalling that in very special geometry, by construction, the quantity

$d_{i j k} \operatorname{Im}\left(z_{H}^{i}\right) \operatorname{Im}\left(z_{H}^{j}\right) \operatorname{Im}\left(z_{H}^{k}\right)$ must have a definite sign, say negative, and this imposes a further constraint on the sign of $x^{i}$ s:

$d_{i j k} \operatorname{Im}\left(z_{H}^{i}\right) \operatorname{Im}\left(z_{H}^{j}\right) \operatorname{Im}\left(z_{H}^{k}\right)<0 \Leftrightarrow-\frac{27}{8} \frac{S^{3}}{\pi^{3}} \frac{d_{i j k} x^{i} x^{j} x^{k}}{\left(\Delta_{l} x^{l}\right)^{3}} \operatorname{sgn}\left(\Delta_{m} x^{m}\right)=-\frac{27}{8} \frac{S^{3}}{\pi^{3}} \frac{d_{i j k} x^{i} x^{j} x^{k}}{\left|\Delta_{l} x^{l}\right|^{3}}<0$.

By exploiting (2.6), condition (2.11) can be rewritten as

$$
-\frac{27 \cdot 6}{8} \frac{S^{3}}{\pi^{3}} \frac{\operatorname{sgn}\left(\Delta_{j} x^{j}\right)}{\left(\Delta_{k} x^{k}\right)^{2}}<0 \Leftrightarrow \Delta_{j} x^{j}>0 \Leftrightarrow \mathcal{V}(x)>0,
$$

and thus (2.10) gets simplified to ${ }^{9}$

$$
z_{H}^{i}\left(p^{0}, p^{k}, q_{0}, q_{k}\right)=\frac{3}{2} \frac{x^{i}}{\Delta_{j} x^{j}}\left[\frac{p^{0}(p \cdot q)-2 I_{3}(p)}{p^{0}}-\mathbf{i} \frac{S}{\pi}\right]+\frac{p^{i}}{p^{0}},
$$

with $S$ given by (2.2). The expression (2.13) highlights the relation between the real and imaginary parts of $z_{H}^{i}$ (i.e., the attractor configurations of axions resp. dilatons) and the $x^{i}$ 's themselves.

\footnotetext{
${ }^{9}$ This formula matches eq. (24) of [122].
} 
All in all, the BPS formulæ (2.2) and (2.13) are well defined for

$$
\operatorname{BPS}:\left\{\begin{array}{l}
\mathcal{V}(x)>0 \Leftrightarrow \Delta_{i} x^{i}>0 \\
4\left(\Delta_{i} x^{i}\right)^{2}-27\left[p^{0}(p \cdot q)-2 I_{3}(p)\right]^{2}>0 .
\end{array}\right.
$$

\section{BPS systems and the gradient map $\nabla_{\mathcal{V}}$}

Given a rank-3 completely symmetric tensor $d_{i j k}=d_{(i j k)}$ in $N$ dimensions $(i, j, k=1, \ldots, N)$, we recall the cubic form defined in (2.8):

$$
\mathcal{V}(x):=\frac{1}{3 !} d_{i j k} x^{i} x^{j} x^{k} .
$$

From the Euler formula, since $\mathcal{V}(3.1)$ is a homogeneous polynomial of degree 3 in the $x$ 's, it follows that

$$
\frac{\partial \mathcal{V}(x)}{\partial x^{i}} x^{i}=3 \mathcal{V}(x)
$$

More subtly, since the $x^{i}$ 's are themselves homogeneous of degree 1 in the black hole charges $p^{i}, p^{0}$ and $q_{i}$, it holds that

$$
\begin{aligned}
& \frac{\partial \mathcal{V}\left(x\left(p^{j}, p^{0}, q_{j}\right)\right)}{\partial p^{i}} p^{i}+\frac{\partial \mathcal{V}\left(x\left(p^{j}, p^{0}, q_{j}\right)\right)}{\partial p^{0}} p^{0}+\frac{\partial \mathcal{V}\left(x\left(p^{j}, p^{0}, q_{j}\right)\right)}{\partial q_{i}} q_{i} \\
= & \frac{\partial \mathcal{V}\left(x\left(p^{j}, p^{0}, q_{j}\right)\right)}{\partial x^{k}} \frac{\partial x^{k}}{\partial p^{i}} p^{i}+\frac{\partial \mathcal{V}\left(x\left(p^{j}, p^{0}, q_{j}\right)\right)}{\partial x^{k}} \frac{\partial x^{k}}{\partial p^{0}} p^{0}+\frac{\partial \mathcal{V}\left(x\left(p^{j}, p^{0}, q_{j}\right)\right)}{\partial x^{k}} \frac{\partial x^{k}}{\partial q_{i}} q_{i} \\
= & \frac{1}{2} d_{k l m} x^{l} x^{m}\left(\frac{\partial x^{k}}{\partial p^{i}} p^{i}+\frac{\partial x^{k}}{\partial p^{0}} p^{0}+\frac{\partial x^{k}}{\partial q_{i}} q_{i}\right)=\frac{1}{2} d_{k l m} x^{k} x^{l} x^{m}=3 \mathcal{V}(x),
\end{aligned}
$$

where we used (2.7).

The BPS system (2.6) can then be defined as the non-homogeneous system of $N$ quadratic equations in $N$ unknowns $x^{i}$ 's $(i=1, \ldots, N)$

$$
\frac{\partial \mathcal{V}(x)}{\partial x^{i}}=3 \frac{\partial \Delta(p, q)}{\partial p^{i}}
$$

where

$$
\Delta(p, q):=\frac{1}{3 !} d_{i j k} p^{i} p^{j} p^{k}-p^{0} p^{i} q_{i}=I_{3}(p)-p^{0} p^{i} q_{i},
$$

is a real quantity depending, for a given $d_{i j k}$, on some real given (background) constants, namely the magnetic and electric charges $p^{i}, p^{0}$ and $q_{i}$ of the extremal black hole. By (3.5), (3.4) can be rewritten as

$$
\frac{1}{2} d_{i j k} x^{j} x^{k}=3 \Delta_{i}(p, q)
$$

where

$$
\Delta_{i}(p, q):=\frac{\partial \Delta(p, q)}{\partial p^{i}}=\frac{1}{2} d_{i j k} p^{j} p^{k}-p^{0} q_{i} .
$$


Thus, by introducing the gradient operators $\nabla_{x}:=\left\{\partial_{x^{i}}\right\}_{i=1, \ldots, N}$ and $\nabla_{p}:=\left\{\partial_{p^{i}}\right\}_{i=1, \ldots, N}$, the BPS system (2.6) (or, equivalently (3.4)) can be cast as follows: ${ }^{10}$

$$
\begin{aligned}
\nabla_{x} \mathcal{V}(x)= & 3 \nabla_{p} \Delta(p, q) ; \\
& \mathbb{1} \\
\partial_{x^{i}} \mathcal{V}(x)= & 3 \Delta_{i}(p, q), \quad \forall i .
\end{aligned}
$$

All quantities involved in these formulæ are real, and, for $N, d_{i j k}$ and $\Delta_{i}(p, q)$ given in input, real solutions $x^{i}=x^{i}\left(\Delta_{j} ; d_{k l m}\right)$ to the system (3.9) are searched in closed form, in such a way that, when plugged into (2.2), one can obtain a closed form expression for the Bekenstein-Hawking entropy (2.2) as well as for the attractor values of scalar fields (2.13) of extremal BPS black holes also in homogeneous non-symmetric very special geometry. Of course, as already mentioned, the system (3.9) might have no analytical real solution in the general case: it describes $N$ quadratic hypersurfaces (each of dimension $N-1$ ) in $\mathbb{R}^{N}$, and the intersection of these hypersurfaces is a solution of the system.

An important quantity is the Hessian matrix of the cubic form $\mathcal{V}(x)(3.1)$ :

$$
H_{i j}(x):=\frac{\partial^{2} \mathcal{V}(x)}{\partial x^{i} \partial x^{j}}=d_{i j k} x^{k}=H_{(i j)}(x),
$$

which can be regarded as the Jacobian matrix of the quadratic map represented by the gradient map $\nabla \mathcal{V}$ of $\mathcal{V}$ itself $^{11}$

$$
\begin{gathered}
\nabla_{\mathcal{V}}: \mathbb{R}^{N} \rightarrow\left(\mathbb{R}^{N}\right)^{*} \simeq \mathbb{R}^{N} \\
(\nabla \mathcal{V})_{i}(x):=\frac{\partial \mathcal{V}(x)}{\partial x^{i}}=\frac{1}{2} d_{i j k} x^{j} x^{k} .
\end{gathered}
$$

Since each coefficient $H_{i j}(x)$ of the symmetric matrix $H$ is a linear homogeneous polynomial in the $x$ 's, the determinant of the $N \times N$ matrix $H(x)$ is a homogeneous polynomial of degree $N$ in the $x$ 's, as evident from (3.10). Even if we will not exploit it in the subsequent treatment, the condition of non-vanishing $\operatorname{det} H(x)$ (i.e. $H(x)$, and thus $\nabla \mathcal{V}$, of maximal rank) can be used to establish whether the gradient map $\nabla_{\mathcal{V}}$ can be inverted; in fact, Dini's Theorem ensures that if $\operatorname{det} H(x) \neq 0$ then locally the map $\nabla \mathcal{V}$ is a diffeomorphism, and therefore $x$ is an isolated point in the fiber $\left(\nabla_{\mathcal{V}}\right)^{-1} \nabla_{\mathcal{V}}(x)$ of $\left(\nabla_{\mathcal{V}}\right)^{-1}$ over $\nabla_{\mathcal{V}}(x)$.

\section{Homogeneous very special geometry}

\subsection{Symmetric $d$-manifolds}

The tensors $d_{i j k}$ as in (2.1) giving rise to homogeneous very special Kähler spaces (which, for this reason, have been named $d$-manifolds) have been classified in [53] (see also $[55,56]$ ). A noteworthy subclass is represented by the symmetric $d$-manifolds [56], whose $d_{i j k}$ 's have

\footnotetext{
${ }^{10}$ We recall that the symplectic bundle of special geometry is flat $[2,38,133]$.

${ }^{11}$ Note that each component of the map $\nabla \mathcal{v}$ is an homogeneous polynomial of degree 2 in the $x$ 's, thus $(\nabla \mathcal{V})_{i}(x)=(\nabla \mathcal{V})_{i}(-x)$
} 
been reconsidered also e.g. in $[30,52,62]$. Symmetric $d$-manifolds are characterized by a purely numerical (constant) contravariant tensor $d^{i j k}$ such that the so-called "adjoint identity" of cubic Jordan algebras holds [47, 77]:

$$
d_{(i j \mid k} d_{l \mid m n)} d^{k l p}=\frac{4}{3} \delta_{(i}^{p} d_{j m n)} .
$$

Thus, when

$$
d^{r s t} \Delta_{r} \Delta_{s} \Delta_{t}>0
$$

a solution to the BPS system (2.6) is given by (see section 3.3.1 of [79])

$$
x^{i}= \pm \frac{3}{\sqrt{2}} \frac{d^{i j k} \Delta_{j} \Delta_{k}}{\sqrt{d^{l m n} \Delta_{l} \Delta_{m} \Delta_{n}}} .
$$

Indeed, by exploiting the adjoint identity (4.1), (4.3) yields to

$$
\frac{1}{3 !} d_{i j k} x^{j} x^{k}=\frac{1}{3 !} \frac{9}{2} \frac{d_{i j k} d^{j l m} d^{k n p} \Delta_{l} \Delta_{m} \Delta_{n} \Delta_{p}}{d^{r s t} \Delta_{r} \Delta_{s} \Delta_{t}} \stackrel{(4.1)}{=} \frac{\delta_{i}^{(l} d^{m n p)} \Delta_{l} \Delta_{m} \Delta_{n} \Delta_{p}}{d^{r s t} \Delta_{r} \Delta_{s} \Delta_{t}}=\Delta_{i}
$$

thus obtaining (2.6).

Furthermore, by using (4.1), one computes that

$$
\begin{aligned}
d^{r s t} \Delta_{r} \Delta_{s} \Delta_{t}= & d^{r s t}\left(\frac{1}{2} d_{r u v} p^{u} p^{v}-p^{0} q_{r}\right)\left(\frac{1}{2} d_{s m n} p^{m} p^{n}-p^{0} q_{s}\right)\left(\frac{1}{2} d_{t p q} p^{p} p^{q}-p^{0} q_{t}\right) \\
= & \frac{1}{8} d^{r s t} d_{r u v} d_{s m n} d_{t p q} p^{u} p^{v} p^{m} p^{n} p^{p} p^{q}-\frac{3}{4} p^{0} d^{r s t} d_{r u v} d_{s m n} p^{u} p^{v} p^{m} p^{n} q_{t} \\
& +\frac{3}{2}\left(p^{0}\right)^{2} d^{r s t} d_{r u v} p^{u} p^{v} q_{s} q_{t}-\left(p^{0}\right)^{3} d^{r s t} q_{r} q_{s} q_{t} \\
= & \frac{1}{6}\left(d_{s m n} p^{s} p^{m} p^{n}\right)^{2}-p^{0} p^{j} q_{j} d_{s m n} p^{s} p^{m} p^{n} \\
& +\frac{3}{2}\left(p^{0}\right)^{2} d^{r s t} d_{r u v} p^{u} p^{v} q_{s} q_{t}-\left(p^{0}\right)^{3} d^{s m n} q_{s} q_{m} q_{n} \\
= & 6\left[I_{3}^{2}(p)-p^{0} p^{j} q_{j} I_{3}(p)+\left(p^{0}\right)^{2}\left\{I_{3}(p), I_{3}(q)\right\}-\left(p^{0}\right)^{3} I_{3}(q)\right]
\end{aligned}
$$

Thus, the consistency condition (4.2) can be recast as follows:

$$
I_{3}^{2}(p)-p^{0} p^{j} q_{j} I_{3}(p)+\left(p^{0}\right)^{2}\left\{I_{3}(p), I_{3}(q)\right\}-\left(p^{0}\right)^{3} I_{3}(q)>0 .
$$

Moreover, the condition (2.12) can be rewritten as

$$
\Delta_{j} x^{j}>0 \Leftrightarrow \pm \frac{3}{\sqrt{2}} \frac{d^{i j k} \Delta_{i} \Delta_{j} \Delta_{k}}{\sqrt{d^{l m n} \Delta_{l} \Delta_{m} \Delta_{n}}}= \pm \frac{3}{\sqrt{2}} \sqrt{d^{l m n} \Delta_{l} \Delta_{m} \Delta_{n}}>0,
$$

implying that only the branch "+" of (4.3) is consistent; thus selecting it (for $\left.d^{l m n} \Delta_{l} \Delta_{m} \Delta_{n}>0\right)$,

$$
x^{i}=\frac{3}{\sqrt{2}} \frac{d^{i j k} \Delta_{j} \Delta_{k}}{\sqrt{d^{l m n} \Delta_{l} \Delta_{m} \Delta_{n}}},
$$


one obtains that the explicit solutions (2.10) to the BPS Attractor Equations read

$$
z_{H}^{i}\left(p^{0}, p^{k}, q_{0}, q_{k}\right)=\frac{3}{2} \frac{d^{i j k} \Delta_{j} \Delta_{k}}{d^{l m n} \Delta_{l} \Delta_{m} \Delta_{n}}\left[\frac{p^{0}(p \cdot q)-2 I_{3}(p)}{p^{0}}-\mathbf{i} \frac{S}{\pi}\right]+\frac{p^{i}}{p^{0}} .
$$

By substituting (4.3) into (2.2), one obtains the BPS entropy $S$ in terms of the quartic invariant polynomial $I_{4}$ (cfr. e.g. [39] and refs. therein), which can be proved to (finitely) generate the ring of invariant polynomials of the non-transitive action of the $4 D$ U-duality group over the black hole charges' representation space [87]. By defining

$$
\begin{aligned}
I_{3}(q) & :=\frac{1}{3 !} d^{i j k} q_{i} q_{j} q_{k} ; \\
\left\{I_{3}(p), I_{3}(q)\right\} & :=\frac{\partial I_{3}(p)}{\partial p^{i}} \frac{\partial I_{3}(q)}{\partial q_{i}}=\frac{1}{4} d^{i j k} d_{i l m} p^{l} p^{m} q_{j} q_{k},
\end{aligned}
$$

and recalling (2.4) and (2.5), the expression (2.2) the BPS entropy can be remarkably simplified into the following formula:

$$
\frac{S}{\pi}=\sqrt{-(p \cdot q)^{2}+4 q_{0} I_{3}(p)-4 p^{0} I_{3}(q)+4\left\{I_{3}(p), I_{3}(q)\right\}}=: \sqrt{I_{4}},
$$

and therefore (4.9) further simplifies to

$$
z_{H}^{i}\left(p^{0}, p^{k}, q_{0}, q_{k}\right)=\frac{3}{2} \frac{d^{i j k} \Delta_{j} \Delta_{k}}{d^{l m n} \Delta_{l} \Delta_{m} \Delta_{n}}\left[\frac{p^{0}(p \cdot q)-2 I_{3}(p)}{p^{0}}-\mathbf{i} \sqrt{I_{4}}\right]+\frac{p^{i}}{p^{0}} .
$$

It should be remarked that the condition $I_{4}>0$ may be weaker than the actual BPS condition; in fact, it satisfied by both BPS and non-BPS attractors (these latter with vanishing central charge). Indeed, in the symmetric $d$-spaces the strictly BPS conditions (2.14) can be specified as the following system of inequalities:

$$
\left\{\begin{array}{l}
I_{4}>0 \Leftrightarrow-(p \cdot q)^{2}+4 q_{0} I_{3}(p)-4 p^{0} I_{3}(q)+4\left\{I_{3}(p), I_{3}(q)\right\}>0 ; \\
d^{i j k} \Delta_{i} \Delta_{j} \Delta_{k}>0 \Leftrightarrow I_{3}^{2}(p)-p^{0} p^{j} q_{j} I_{3}(p)+\left(p^{0}\right)^{2}\left\{I_{3}(p), I_{3}(q)\right\}-\left(p^{0}\right)^{3} I_{3}(q)>0 .
\end{array}\right.
$$

\section{$4.2 \quad$ Non-symmetric $d$-manifolds}

In non-symmetric $d$-manifolds, the tensor $d^{i j k}$ still exists, ${ }^{12}$ but it generally depends on the rescaled imaginary parts (denoted below by $\hat{\lambda}^{i}$ ) of the scalar fields $z^{i}$; indeed, within the conventions of [39], the "dual" $d^{i j k}$ cubic tensor is generally defined as

$$
\begin{aligned}
d^{i j k} & :=a^{i l} a^{j m} a^{k n} d_{l m n} ; \\
a^{i j} & :=\frac{1}{2}\left(\hat{\lambda}^{i} \hat{\lambda}^{j}-2 \hat{\kappa}^{i j}\right),
\end{aligned}
$$

\footnotetext{
${ }^{12}$ As mentioned above, for symmetric $d$-spaces, it should hold that $\frac{\partial d^{i j k}}{\partial \hat{\lambda}^{l}}=0$, and this can be checked by exploiting the explicit expression of $d_{i j k}$ in such cases $[53,55,56,62]$.
} 
where the scalar fields read

$$
\begin{aligned}
z^{i} & =: x^{i}-i \mathcal{V}^{1 / 3} \hat{\lambda}^{i} ; \\
\frac{1}{3 !} d_{i j k} \hat{\lambda}^{i} \hat{\lambda}^{j} \hat{\lambda}^{k} & =: 1,
\end{aligned}
$$

and $\hat{\kappa}^{i j}$ is the inverse matrix of

$$
\hat{\kappa}_{j k}:=d_{j k l} \hat{\lambda}^{l}, \quad \hat{\kappa}^{i j} \hat{\kappa}_{j k}=: \delta_{k}^{i} .
$$

Thus, it generally holds that

$$
\frac{\partial d^{i j k}}{\partial \hat{\lambda}^{l}} \neq 0
$$

This can also be obtained by the generalization of the adjoint identity (4.1) in non-symmetric very special geometry, ${ }^{13}$ which reads [30]:

$$
d_{(i j \mid k} d_{l \mid m n)} d^{k l p}=\frac{4}{3} \delta_{(i}^{p} d_{j m n)}+E^{p}{ }_{i j m n},
$$

where the so-called "E-tensor" is defined as [29]

$$
\begin{aligned}
& E^{p}{ }_{i j m n}=a^{p k} E_{p \mid i j m n} ; \\
& E_{p \mid i j m n}=E_{p \mid(i j m n)}:=-\frac{1}{12}\left[\begin{array}{c}
\left(4 \hat{\kappa}_{(i} d_{j m n)}-3 \hat{\kappa}_{(i j} \hat{\kappa}_{m n)}\right) \hat{\kappa}_{p}+12 d_{p(i j} \hat{\kappa}_{m n)} \\
-16 \hat{\kappa}_{p(i} d_{j m n)}-12 d_{q(i j} d_{m n) r} d_{p s t} \hat{\kappa}^{q s} \hat{\kappa}^{r t}
\end{array}\right],
\end{aligned}
$$

where

$$
\hat{\kappa}_{i}:=\hat{\kappa}_{i j} \hat{\lambda}^{j}=d_{i j k} \hat{\lambda}^{j} \hat{\lambda}^{k}
$$

\subsection{Classification of homogeneous $d$-manifolds: $L(q, P, \dot{P})$}

In the present investigation, we will focus on the solution of the BPS system (2.6) in the case in which the $d$-manifolds (coupled to $\mathcal{N}=2, D=4$ supergravity) are homogeneous (and thus the $U$-duality group has a non-linear but transitive action on the target space of scalar fields) but ${ }^{14}$ non-symmetric. ${ }^{15}$ To this aim, we now recall some basic facts on the classification of homogeneous $d$-spaces.

In [53, 55, 56], homogeneous very special Kähler spaces arising as non-compact Riemannian scalar manifolds of vector multiplets in $\mathcal{N}=2, D=4$ supergravity have been classified. ${ }^{16}$ They are denoted as " $L$-spaces" (or " $L$-models"), specified by three sets of

\footnotetext{
${ }^{13}$ At least in the homogeneous non-symmetric case, such a generalization can be regarded as the "generalized adjoint identity" holding for the Hermitian part of the rank-3 Vinberg's T-algebras [135].

${ }^{14}$ Notice that all (known and classified) homogeneous non-symmetric manifolds are of $d$-type, even if, as far as we know, there is no proof that homogeneous non-symmetricity implies $d$-type.

${ }^{15}$ Within the black hole effective potential formalism, a discussion of the various classes of attractors and related black hole entropies in homogeneous scalar manifolds of $\mathcal{N}=2, D=4$ supergravity has been given in [51]. Therein, in (2.23)-(2.25) the explicit expression of the $E$-tensor (which is non-vanishing for homogeneous non-symmetric cases) has been computed.

${ }^{16}$ In [43] this classification has been rephrased in terms of normal $J$-algebras; for a recent survey, see also [12].
} 
integer parameters: $L(q, P, \dot{P})$. In such a classification, the index $i=1, \ldots, N$ is partitioned as ${ }^{17}$

$$
\begin{aligned}
\{i\} & =s,\{I\},\{\alpha\} ; \\
I & =0,1, \ldots, q+1 ; \\
\alpha & =1, \ldots, \mathcal{D}_{q+1} \cdot(P+\dot{P}) ; \\
q & \in \mathbb{N} \cup\{0,-1\}, P, \dot{P} \in \mathbb{N} \cup\{0\},
\end{aligned}
$$

where $\mathcal{D}_{q+1}$ is a certain function of $q$ valued in $\mathbb{N}$ (see e.g. table 1 of [53]):

$\begin{array}{cc}q & \mathcal{D}_{q+1} \\ -1 & 1 \\ 0 & 1 \\ 1 & 2 \\ 2 & 4 \\ 3 & 8 \\ 4 & 8 \\ 5 & 16 \\ 6 & 16 \\ 7 & 16 \\ \mathbf{n}+7 & 16 \cdot \mathcal{D}_{\mathbf{n}}\end{array}$

Note that we set

$$
N=q+3+\mathcal{D}_{q+1} \cdot(P+\dot{P})
$$

The electric-magnetic $(U$-)duality group $\mathcal{G}$ of the $L(q, P, \dot{P})$ models has the following graded structure $[53,55]$ :

$$
\begin{aligned}
\mathcal{G} & =\mathcal{G}_{0} \ltimes\left(\mathcal{G}_{1} \times \mathcal{G}_{2}\right), \\
\mathcal{G}_{0} & =\left(S O(q+2,2) \times S_{q}(P, \dot{P}) \times \mathrm{SO}(1,1)\right)_{0} ; \\
\mathcal{G}_{1} & =\left(\psi_{q+2,2}, \mathbf{F}_{q, P, \dot{P}}\right)_{1} ; \\
\mathcal{G}_{2} & =(\mathbf{1}, \mathbf{1})_{2},
\end{aligned}
$$

where the subscripts (outside round brackets) denote the weights w.r.t. the $\mathrm{SO}(1,1)$ factor in $\mathcal{G}_{0}$. Moreover, $S_{q}(P, \dot{P})$ is the compact, metric-preserving group in the centralizer of the real Euclidean Clifford algebra $C l(q+1,0)$ in the $\mathcal{D}_{q+1}(P+\dot{P})$-dimensional representation

\footnotetext{
${ }^{17}$ In the present paper, attention should be paid to the three different uses of ' $'$ : it indicates a scalar product involving naught and $i$-indices (as in (2.4)), or an algebraic multiplication (as in the third row of (4.25)), or a scalar product involving only $i$-indices (split into $s$, and $x$ - and $y$-indices), as in (6.30)-(6.33). We hope that such different meanings are easily inferred from the context.
} 
(see e.g. in table 3 of [56]),

$\begin{array}{cc}q & S_{q}(P, \dot{P}) \\ -1 & \mathrm{SO}(P) \\ 0 & \mathrm{SO}(P) \otimes \mathrm{SO}(\dot{P}) \\ 1 & \mathrm{SO}(P) \\ 2 & \mathrm{U}(P) \\ 3 & \mathrm{USp}(2 P) \\ 4 & \mathrm{USp}(2 P) \otimes \mathrm{USp}(2 \dot{P}) \\ 5 & \mathrm{USp}(2 P) \\ 6 & \mathrm{U}(P) \\ 7 & \mathrm{SO}(P) \\ \mathbf{n}+7 & \text { as for } q+1=\mathbf{n} .\end{array}$

Also, $\psi_{q+2,2}$ denotes the (semi)spinor representation of $\operatorname{Spin}(q+2,2)$, and $\mathbf{F}_{q, P, \dot{P}}$ stands for the fundamental representation of $S_{q}(P, \dot{P})$ itself.

The vector multiplets' scalar manifold is a non-symmetric projective special Kähler, non-compact Riemannian coset of the form

$$
\mathcal{M}_{4}:=\mathcal{G} / H
$$

where $H$ is the maximal compact subgroup of $\mathcal{G}_{0}$ itself,

$$
H=m c s\left(\mathcal{G}_{0}\right)=S O(q+2) \times S_{q}(P, \dot{P}) \times \mathrm{SO}(2) .
$$

By respectively denoting with $\mathfrak{g}$ and $\mathfrak{h}$ the Lie algebras of the groups $G$ (4.28)-(4.31) and $H$ (4.34), the scalar fields coordinatizing $\mathcal{M}_{4}$ group into $H$-representations, such that the Lie algebra of the coset $\mathcal{M}_{4}$ enjoys the following 5 -graded structure, ${ }^{18}$

$$
\begin{aligned}
\operatorname{Lie}\left(\mathcal{M}_{4}\right)= & \mathfrak{g} \ominus \mathfrak{h} \\
= & (\mathbf{q}+\mathbf{2}, \mathbf{1})_{0,-2} \oplus\left(\psi_{q+2}^{(\prime)}, \mathbf{F}_{q, P, \dot{P}}\right)_{1,-1} \oplus(\mathbf{1}, \mathbf{1})_{0,0} \oplus(\mathbf{1}, \mathbf{1})_{2,0} \oplus\left(\psi_{q+2}, \mathbf{F}_{q, P, \dot{P}}\right)_{1,1} \\
& \oplus(\mathbf{q}+\mathbf{2}, \mathbf{1})_{0,2},
\end{aligned}
$$

where the first subscript reports (as above) the weight w.r.t. the $\operatorname{SO}(1,1)$ factor in $\mathcal{G}_{0}$, whereas the second subscript denotes the charge w.r.t. the $\mathrm{SO}(2) \simeq \mathrm{U}(1)$ factor in $H(4.34)$. The total (real) dimension of $\mathcal{M}_{4}$ is then

$$
\operatorname{dim} \mathcal{M}_{4}=\operatorname{dim} \operatorname{Lie}\left(\mathcal{M}_{4}\right)=2+2(q+2)+2 \mathcal{D}_{q+1}(P+\dot{P})=2 \operatorname{dim} \mathcal{M}_{5}+2,
$$

where

$$
\operatorname{dim} \mathcal{M}_{5}=q+2+\mathcal{D}_{q+1}(P+\dot{P})
$$

is the (real) dimension of the vector multiplets' real scalar manifold of the parent theory in 5 space-time (Lorentzian) dimensions [53, 55].

\footnotetext{
${ }^{18}$ Note that the presence of the upperscript " (')" means that (4.35) and (4.38) hold up to possible spinor conjugation of (semi)spinors $\psi$.
} 
The 2 -form field strengths and their duals fit into the (generally reducible) $\mathcal{G}_{0}$-module

$$
\mathbf{R}_{4}:=(\mathbf{q}+\mathbf{4}, \mathbf{1})_{-1} \oplus\left(\psi_{q+2,2}^{(\prime)}, \mathbf{F}_{q, P, \dot{P}}\right)_{0} \oplus(\mathbf{q}+\mathbf{4}, \mathbf{1})_{1}
$$

which has thus a 3 -graded structure w.r.t. the $\mathrm{SO}(1,1)$ factor in $\mathcal{G}_{0}$. The $\mathcal{G}_{0}$-module (4.38), of real dimension

$$
\operatorname{dim} \mathbf{R}_{4}=2(q+4)+2 \mathcal{D}_{q+1}(P+\dot{P})
$$

is anti-self-conjugate (i.e., it is symplectic), and the corresponding ring of $\mathcal{G}$-invariant homogeneous polynomials is one-dimensional (cfr. the discussion in [12]). As far as we know, the expression (4.38) has never been presented in the literature thus far.

The non-vanishing components of $d_{i j k}=d_{(i j k)}$ are

$$
\frac{1}{3 !} d_{i j k}:\left\{\begin{array}{l}
\frac{1}{3 !} d_{s I J}:=\eta_{I J}=\operatorname{diag}(-1, \underbrace{1, \ldots, 1}_{q+1}) \\
\frac{1}{3 !} d_{I \alpha \beta}:=\left(\Gamma_{I}\right)_{\alpha \beta},
\end{array}\right.
$$

where, for $I=0,1, \ldots, q+1$, the square matrices $\Gamma_{I}$, of size $\mathcal{D}_{q+1} \cdot(P+\dot{P})$ and components $\left(\Gamma_{I}\right)_{\alpha \beta}$ are defined as follows: $\Gamma_{0}:=\mathbb{I}_{\mathcal{D}_{q+1} \cdot(P+\dot{P})}$, and $\left\{\Gamma_{I}\right\}_{I \neq 0}$ are $\Gamma$-matrices that provide a real representation of the Euclidean Clifford algebra $C l(q+1,0)$; see section 5 . All the other, unwritten, components of $d_{i j k}$ vanish. We should also recall that non-vanishing (and independent) values of $\dot{P}$ are possible only when $q=4 m$, with $m \in \mathbb{N} \cup\{0\}$. Indeed, for $q=0$ $\bmod 4$ the representations $\Gamma_{I}$ and $-\Gamma_{I}$ are not equivalent, and a reducible representation is given by $\Gamma=\eta \otimes \Gamma_{I}($ with $\eta=\operatorname{diag}(\underbrace{1, \ldots, 1}_{P \text { times }}, \underbrace{-1, \ldots,-1}_{\dot{P} \text { times }})$ ) and thus characterized by the multiplicity of each of these representations, namely $P$ and $\dot{P}$; of course, an overall sign change of all the gamma matrices can always be re-absorbed, and this is the reason why $L(4 m, P, \dot{P})=L(4 m, \dot{P}, P)$. If the representation consists of copies of only one version of the irreducible representations, then we denote it by $L(4 m, P)$.

In correspondence with the splitting (4.25) of the index set, we introduce new variables $s, x^{I}$ and $y^{\alpha}$, and define

$$
\xi:={ }^{T}\left(s, x^{I}, y^{\alpha}\right)
$$

Then, (3.1) and (3.5) can be written as

$$
\begin{aligned}
\mathcal{V}(x) & =s \eta_{I J} x^{I} x^{J}+x^{I}\left(\Gamma_{I}\right)_{\alpha \beta} y^{\alpha} y^{\beta}=s q(x)+x^{I} Q_{I}(y) \\
\Delta(p, q) & =\mathcal{V}(p)-p^{0}\left(p^{s} q_{s}+p^{I} q_{I}+p^{\alpha} q_{\alpha}\right) \\
& =p^{s} \eta_{I J} p^{I} p^{J}+p^{I}\left(\Gamma_{I}\right)_{\alpha \beta} p^{\alpha} p^{\beta}-p^{0}\left(p^{s} q_{s}+p^{I} q_{I}+p^{\alpha} q_{\alpha}\right),
\end{aligned}
$$


where $^{19}$

$$
\begin{aligned}
q(x) & :=\eta_{I J} x^{I} x^{J} ; \\
Q_{I}(y) & :=\left(\Gamma_{I}\right)_{\alpha \beta} y^{\alpha} y^{\beta} .
\end{aligned}
$$

The BPS system (2.6) (or, equivalently, (3.9)) is an inhomogeneous system of $N$ quadratic equations in $N$ unknowns $\left(s, x^{I}, y^{\alpha}\right)$, and it acquires the following form:

$$
\left\{\begin{array}{l}
q(x)=3 \Delta_{s} ; \\
2 s \eta_{I J} x^{J}+Q_{I}(y)=3 \Delta_{I} \\
2 x^{I}\left(\Gamma_{I}\right)_{\alpha \beta} y^{\beta}=3 \Delta_{\alpha} .
\end{array}\right.
$$

The open orbit of electric and magnetic charges supporting $\frac{1}{2}$-BPS "large" extremal black holes is an homogeneous non-symmetric manifold,

$$
\mathcal{O}_{\mathrm{BPS}}=\frac{\mathcal{G}}{H_{\mathrm{BPS}}}, \quad \text { with } H_{\mathrm{BPS}}=S O(q+2) \times S_{q}(P, \dot{P}),
$$

of real dimension

$$
\operatorname{dim} \mathcal{O}_{\mathrm{BPS}}=2 q+7+2 \mathcal{D}_{q+1}(P+\dot{P}) \stackrel{(4.39)}{=} \operatorname{dim} \mathbf{R}_{4}-1 .
$$

Since $\mathcal{O}_{\mathrm{BPS}}$ is a generic, open orbit of the non-transitive action of the Lie group $\mathcal{G}$ (4.28)-(4.31) on the representation space $\mathbf{R}_{4}$, the result (4.48) is consistent with the general counting formula:

$$
\operatorname{dim} \mathbf{R}_{4}=\operatorname{dim} \mathcal{O}_{\mathrm{BPS}}+1,
$$

because, as mentioned above, the ring of $\mathcal{G}$-invariant homogeneous polynomials on $\mathbf{R}_{4}$ is one-dimensional [12]. Moreover, the fact that the stabilizer $H_{\mathrm{BPS}}(4.47)$ of $\mathcal{O}_{\mathrm{BPS}}$ is compact implies that no moduli space of BPS attractors exists at all, as pointed out in [63] and firstly noticed in [61].

\subsection{1 $L(q, 1)$ and "magic" enhancements}

A noteworthy subclass of homogeneous $d$-manifolds is provided by the models $L(q, 1,0) \equiv$ $L(q, 1)$ [53], which have $P=1$ and $\dot{P}=0$. From (4.28)-(4.31), the electric-magnetic $(U$-)duality group $\mathcal{G}$ of the $L(q, 1)$ models has the following graded structure:

$$
\begin{aligned}
\mathcal{G} & =\mathcal{G}_{0} \ltimes\left(\mathcal{G}_{1} \times \mathcal{G}_{2}\right), \\
\mathcal{G}_{0} & =\left(S O(q+2,2) \times S_{q}(1,0) \times \mathrm{SO}(1,1)\right)_{0} ; \\
\mathcal{G}_{1} & =\left(\psi_{q+2,2}, \mathbf{F}_{q, 1,0}\right)_{1} ; \\
\mathcal{G}_{2} & =(\mathbf{1}, \mathbf{1})_{2},
\end{aligned}
$$

\footnotetext{
${ }^{19}$ The parameter $q \in \mathbb{N} \cup\{0,-1\}$ occurring in de Wit-Van Proeyen's classification [53] should not be confused with the quadratic form $q(x)(4.44)$.
} 
where the subscripts (outside round brackets) denote the weights w.r.t. the $\mathrm{SO}(1,1)$ factor in $\mathcal{G}_{0}$. Moreover, $S_{q}(1,0)$ is the compact, metric-preserving group in the centralizer of the real Euclidean Clifford algebra $C l(q+1,0)$ in the $\mathcal{D}_{q+1}$-dimensional representation,

$\begin{array}{cc}q & S_{q}(1,0) \\ -1 & \mathbb{I} \\ 0 & \mathbb{I} \\ 1 & \mathbb{I} \\ 2 & \mathrm{U}(1) \\ 3 & \mathrm{USp}(2) \\ 4 & \mathrm{USp}(2) \\ 5 & \mathrm{USp}(2) \\ 6 & \mathrm{U}(1) \\ 7 & \mathbb{I} \\ \mathbf{n}+7 \text { as for } q+1=\mathbf{n},\end{array}$

where $\mathbb{I}$ denotes the identity. It is interesting to note that for $q=1,2,4,8$, the Lie algebra of $S_{q}(1,0)=\mathbb{I}, \mathrm{U}(1), \mathrm{USp}(2), \mathbb{I}$ can be regarded as

$$
\operatorname{Lie}\left(S_{q}(1,0)\right)=\varnothing, \mathfrak{u}_{1}, \mathfrak{u s p}_{2}, \varnothing=\mathfrak{t r i}(\mathbb{A}) \ominus \mathfrak{s o}(\mathbb{A}), \text { with } \mathbb{A}=\mathbb{R}, \mathbb{C}, \mathbb{H}, \mathbb{O},
$$

respectively. "trit" and "so" respectively denote the triality and norm-preserving Lie algebras of the corresponding division algebra $\mathbb{A}$ (see e.g. [13]).

Furthermore, the underlying real vector space $\mathbf{V}$ has the following structure:

$$
\mathbf{V}=\mathbb{R} \oplus V \oplus \mathcal{S}, \quad \operatorname{dim} V=q+2, \quad \operatorname{dim} \mathcal{S}=\mathcal{D}_{q+1}
$$

where $\mathcal{D}_{q+1}$ is the minimal dimension for which there are Clifford matrices $\Gamma_{1}, \ldots, \Gamma_{q+1} \in$ $M_{\mathcal{D}_{q+1}}(\mathbb{R})$, and it is given in (4.26) (cfr. also table 1 of [56] or table 3 of [56]). The total dimensions of $\mathbf{V}$ is thus $1+(q+2)+\mathcal{D}_{q+1}$. By defining the standard quadratic form in $\mathbb{R}^{h}$ as

$$
q_{h}(x):=\left(x^{1}\right)^{2}+\ldots+\left(x^{h}\right)^{2}=\sum_{I=1}^{h}\left(x^{I}\right)^{2},
$$

there exists a Lorentzian (mostly plus) quadratic form on $V$ :

$$
q\left(x^{0}, x^{1}, \ldots, x^{q+1}\right):=-\left(x^{0}\right)^{2}+q_{q+1}(x):=-\left(x^{0}\right)^{2}+\left(x^{1}\right)^{2}+\ldots+\left(x^{q+1}\right)^{2} .
$$

On the other hand, the $\Gamma$-matrices ${ }^{20}$ of the Euclidean Clifford algebra $C l(q+1,0)$ will enter (only) as the matrices defining quadratic forms $Q_{I}$ on $\mathcal{S}$, and they will all be symmetric matrices [53]. In a different context, these models have been treated in [6].

In $L(q, 1)$ models, the 2 -form field strengths and their duals fit into the (generally reducible) 3 -graded $\left(S O(q+2,2) \times S_{q}(1,0) \times \mathrm{SO}(1,1)\right)$-module

$$
\mathbf{R}_{4}:=(\mathbf{q}+\mathbf{4}, \mathbf{1})_{-1} \oplus\left(\psi_{q+2,2}^{(\prime)}, \mathbf{F}_{q, 1,0}\right)_{0} \oplus(\mathbf{q}+\mathbf{4}, \mathbf{1})_{1} .
$$

\footnotetext{
${ }^{20}$ Notice that one can take "diagonal blocks" of $\Gamma$-matrices to produce new ones.
} 
The open orbit of electric and magnetic charges supporting $\frac{1}{2}$-BPS "large" extremal black holes reads

$$
\mathcal{O}_{\mathrm{BPS}}=\frac{\mathcal{G}}{H_{\mathrm{BPS}}}, \quad \text { with } H_{\mathrm{BPS}}=S O(q+2) \times S_{q}(1,0),
$$

with real dimension

$$
\operatorname{dim} \mathcal{O}_{\mathrm{BPS}}=2 q+7+2 \mathcal{D}_{q+1}
$$

Within the class $L(q, 1)$, there are special models corresponding to homogeneous symmetric $d$-manifolds: they occur when, for $\mathcal{D}_{q+1}=2^{g}=2,4,8,16$, with $g=1,2,3,4$, the maximal corresponding $q$, namely $q=2^{g-1}=1,2,4,8$ such that $\mathcal{D}_{q+1}=2 q$, is taken; the corresponding models, named "magic" [76-78], all pertain to $\mathcal{N}=2$ Maxwell-Einstein supergravity:

$$
\begin{array}{cc}
L(1,1), & L(2,1), \\
\operatorname{dim} \mathbf{V}=1+3+2=6, & \operatorname{dim} \mathbf{V}=1+4+4=9, \\
C l(2,0), & C l(3,0), \\
J_{3}^{\mathbb{R}} ; & J_{3}^{\mathbb{C}} ; \\
L(4,1), & L(8,1), \\
\operatorname{dim} \mathbf{V}=1+6+8=15, & \operatorname{dim} \mathbf{V}=1+10+16=27, \\
C l(5,0), & C l(9,0), \\
J_{3}^{\mathbb{H}} ; & J_{3}^{\mathbb{Q}},
\end{array}
$$

respectively. In these models, $\mathbf{V}$ has dimension $3 q+3$, and it can be realized as the simple cubic Jordan algebra $J_{3}^{\mathbb{A}}$ of Hermitian $3 \times 3$ matrices over the division algebra division algebra $\mathbb{A}=\mathbb{R}, \mathbb{C}, \mathbb{H}, \mathbb{O}$, with $\mathbb{O}$ denoting the algebra of octonions [86]. As we have already mentioned (cfr. section 4.1), the entropy of extremal black holes in these models is explicitly known, and it is given by the (unique) quartic invariant polynomial, $I_{4}$. The symmetries of "magic" Maxwell-Einstein supergravity theories in $D=3,4$ and 5 space-time dimensions have been discussed along the years from various perspectives, see e.g. [66, 70-72] (and refs. therein).

It is interesting to remark that the $J_{3}^{\mathbb{A}}$-based "magic" models $L(q, 1)$ with $q=1,2,4,8$ correspond to "sweet spots" within the class $L(q, 1)$; in fact, for $q=1,2,4,8$ an enhancement of the electric-magnetic $\left(U\right.$-)duality group $\mathcal{G}$ (4.50)-(4.53), as well as of its $\mathbf{R}_{4}$ module (4.59) and of the corresponding BPS orbit $\mathcal{O}_{\mathrm{BPS}}(4.60)$ ), takes place (for further detail, see e.g. the discussion on triality in [104], as well as [16] for enhanced BPS orbits). It should be noticed that the dimension of the scalar manifold as well as of the BPS "large" charge orbit is invariant under such an enhancement, because the same amount of (compact) generators is added at the numerator and at the denominator of the corresponding cosets. Explicitly, one has:

- $q=1: L(q, 1) \leftrightarrow J_{3}^{\mathbb{R}}$,

$$
\begin{array}{ccc}
\mathcal{G}: & (S O(3,2) \times \mathrm{SO}(1,1))_{0} \ltimes\left(\mathbf{4}_{1} \times \mathbf{1}_{2}\right) & \longrightarrow \mathrm{Sp}(6, \mathbb{R}) ; \\
\mathbf{R}_{4}: & \mathbf{5}_{-1} \oplus \mathbf{4}_{0} \oplus \mathbf{5}_{1} & \longrightarrow \mathbf{1 4}^{\prime} \equiv \wedge_{0}^{3} \\
\mathcal{O}_{\mathrm{BPS}}: & \frac{(S O(3,2) \times \mathrm{SO}(1,1))_{0} \times\left(\mathbf{4}_{1} \times \mathbf{1}_{2}\right)}{\mathrm{SO}(3) \times \mathrm{SO}(2)} & \longrightarrow \frac{\mathrm{Sp}(6, \mathbb{R})}{\mathrm{SU}(3)}
\end{array}
$$


- $q=2: L(q, 2) \leftrightarrow J_{3}^{\mathbb{C}}$,

$$
\begin{array}{cccc}
\mathcal{G}: & (S O(4,2) \times \mathrm{U}(1) \times \mathrm{SO}(1,1))_{0,0} \times\left(\mathbf{4}_{1,1} \times \mathbf{4}_{1,-1}^{\prime} \times \mathbf{1}_{2,0}\right) & \longrightarrow \mathrm{SU}(3,3) ; \\
\mathbf{R}_{4}: & \mathbf{6}_{-1,0} \oplus \mathbf{4}_{0,1} \oplus \mathbf{4}_{0,-1}^{\prime} \oplus \mathbf{6}_{1,0} & \longrightarrow \mathbf{2 0} \equiv \wedge^{3} ; \\
\mathcal{O}_{\mathrm{BPS}}: & \frac{(S O(4,2) \times \mathrm{U}(1) \times \mathrm{SO}(1,1))_{0,0} \times\left(\mathbf{4}_{1,1} \times \mathbf{4}_{1,-1}^{\prime} \times \mathbf{1}_{2,0}\right)}{\mathrm{SO}(4) \times \mathrm{SO}(2) \times \mathrm{U}(1)} & \longrightarrow \frac{\mathrm{SU}(3,3)}{\mathrm{SU}(3) \times \mathrm{SU}(3)}
\end{array}
$$

- $q=4: L(q, 4) \leftrightarrow J_{3}^{\mathbb{H}}$,

$$
\begin{array}{cccc}
\mathcal{G}: & (S O(6,2) \times \mathrm{USp}(2) \times \mathrm{SO}(1,1))_{0} \times\left(\left(\mathbf{8}_{s}, \mathbf{2}\right)_{1} \times(\mathbf{1}, \mathbf{1})_{2}\right) & \longrightarrow S O^{*}(12) ; \\
\mathbf{R}_{4}: & \left(\mathbf{8}_{v}, \mathbf{1}\right)_{-1} \oplus\left(\mathbf{8}_{c}, \mathbf{2}\right)_{0} \oplus\left(\mathbf{8}_{v}, \mathbf{1}\right)_{1} & \longrightarrow \mathbf{3 2}^{(\prime)} \\
\mathcal{O}_{\mathrm{BPS}}: & \frac{(S O(6,2) \times \mathrm{USp}(2) \times \mathrm{SO}(1,1))_{0} \times\left(\left(\mathbf{8}_{s}, \mathbf{2}\right)_{1} \times(\mathbf{1}, \mathbf{1})_{2}\right)}{\mathrm{SO}(6) \times \mathrm{SO}(2) \times \mathrm{USp}(2)} & & \\
& & & \frac{S O^{*}(12)}{\mathrm{SU}(6)}
\end{array}
$$

- $q=8: L(q, 8) \leftrightarrow J_{3}^{\mathbb{Q}}$,

$$
\begin{array}{cccc}
\mathcal{G}: & (S O(10,2) \times \mathrm{SO}(1,1))_{0} \ltimes\left(\mathbf{3 2}_{1} \times \mathbf{1}_{2}\right) & \longrightarrow E_{7(-25)} \\
\mathbf{R}_{4}: & \mathbf{1 2}_{-1} \oplus \mathbf{3 2}_{0} \oplus \mathbf{1 2}_{1} & \longrightarrow & \mathbf{5 6} ; \\
\mathcal{O}_{\mathrm{BPS}}: & \frac{(S O(10,2) \times \mathrm{SO}(1,1))_{0} \ltimes\left(\mathbf{3 2}_{1} \times \mathbf{1}_{2}\right)}{\mathrm{SO}(10) \times \mathrm{SO}(2)} & \longrightarrow \frac{E_{7(-25)}}{E_{6(-78)}}
\end{array}
$$

Finally, we remark that, while for $0 \leqslant q \leqslant 8$ the $L(q, 1)$ models are sub-models obtainable by suitable truncation of the exceptional "magic" Maxwell-Einstein $\mathcal{N}=2$, $D=4$ supergravity model $L(8,1)$ based on $J_{3}^{\mathbb{Q}}$, for $q \geqslant 9$ such models provide an infinite (countable) sequence of homogeneous non-symmetric models which are not truncations of any symmetric model.

\section{Basics on Euclidean Clifford algebras}

We recall the definition of a Clifford algebra and the basic results on (matrix) representations of such algebras. We then discuss $\Gamma$-matrices and Clifford sets of $\Gamma$-matrices, which define such representations. We recall the Pauli matrices and introduce quadratic forms defined by symmetric $\Gamma$-matrices that are tensor products of Pauli matrices. These quadratic forms are building blocks in the definition of the cubic forms in the $L(q, P, \dot{P})$-models.

\subsection{Euclidean Clifford algebras}

The Euclidean Clifford algebra $C l(n, 0)$ is the quotient of the tensor algebra on $V=\mathbb{R}^{n}$ by the relations $q_{n}(v)=v \otimes v$ for all $v \in V$, where $q_{n}(v)$ is the Euclidean quadratic form (4.57):

$$
C l(n, 0) \equiv C l\left(V, q_{n}\right):=\left(\oplus_{k=0}^{\infty} V^{\otimes k}\right) /<q_{n}(v)-v \otimes v>.
$$

We identify $V$ with the corresponding subspace in $C l(n, 0)$ and we write $x y$ for the product of $x$ and $y \in C l(n, 0)$. If $e_{1}, \ldots, e_{n}$ is an orthonormal basis of $V$ and we write $v=$ $x^{1} e_{1}+\ldots+x^{n} e_{n}$, then in $C l(n, 0)$ we have $q_{n}(v)=v^{2}$, so:

$$
\left(x^{1}\right)^{2}+\ldots+\left(x^{n}\right)^{2}=\left(x^{1} e_{1}+\ldots+x^{n} e_{n}\right)^{2},
$$


and expanding the right hand side we see that in $C l(n, 0)$ we have (here $I, J=1, \ldots, n$ )

$$
e_{I}^{2}=1, \quad e_{I} e_{J}+e_{J} e_{I}=0 \quad(I \neq J) .
$$

The Euclidean Clifford algebra $C l(n, 0)$ is a vector space of dimension $2^{n}$ with basis the $e_{I_{1}} e_{I_{2}} \ldots e_{I_{k}}$ with $I_{1}<I_{2}<\ldots<I_{k}$ and $k=1,2, \ldots, n$.

\subsection{Representations of $\mathrm{Cl}(n, 0)$ and Clifford sets}

The structure of the Euclidean Clifford algebra $C l(n, 0)$ is well-known, we just recall here the 'Bott periodicity': denoting by $M_{16}(\mathbb{R})=\mathbb{R}(16)$ the algebra of real $16 \times 16$ matrices (of dimension $16 \times 16=2^{8}$ ), it holds that

$$
C l(n+8,0) \cong C l(n, 0) \otimes M_{16}(\mathbb{R}) .
$$

For $n=1, \ldots, 8$, there are isomorphisms of algebras:

$$
C l(1,0) \cong \mathbb{R} \times \mathbb{R}, \quad C l(2,0) \cong M_{2}(\mathbb{R}), \quad C l(3,0) \cong M_{2}(\mathbb{C}), \quad C l(4,0) \cong M_{2}(\mathbb{H}),
$$

where $\mathbb{H}$ is the division algebra of quaternions,

$$
\begin{array}{lll}
C l(5,0) \cong M_{2}(\mathbb{H}) \times M_{2}(\mathbb{H}), & & C l(6,0) \cong M_{4}(\mathbb{H}) \\
C l(7,0) \cong M_{8}(\mathbb{C}), & & C l(8,0) \cong M_{16}(\mathbb{R})
\end{array}
$$

Bott periodicity then implies that

$$
C l(9,0) \cong M_{16}(\mathbb{R}) \times M_{16}(\mathbb{R}), \quad C l(10,0) \cong M_{2}(\mathbb{R}) \otimes M_{16}(\mathbb{R}) \cong M_{32}(\mathbb{R}) .
$$

Since $\mathbb{C}$ and $\mathbb{H}$ can be identified with subalgebras of $M_{2}(\mathbb{R})$ and $M_{4}(\mathbb{R})$ respectively, we obtain non-trivial homomorphisms (which are injective unless $n=1 \bmod 4$, and in that case they are projections on one of the two factors):

$$
C l(1,0) \rightarrow \mathbb{R}, \quad C l(2,0) \rightarrow M_{2}(\mathbb{R}), \quad C l(3,0) \rightarrow M_{4}(\mathbb{R}), \quad C l(4,0), C l(5,0) \rightarrow M_{8}(\mathbb{R}),
$$

and similarly

$$
C l(6,0), C l(7,0), C l(8,0), C l(9,0) \rightarrow M_{16}(\mathbb{R}) .
$$

Notice that these are homomorphisms $C l(q+1,0) \rightarrow M_{\mathcal{D}_{q+1}}(\mathbb{R})$ with $\mathcal{D}_{q+1}$ as in (4.26).

Under the homomorphisms (5.9) and (5.10), the elements $e_{I} \in C l(n, 0)$ map to so-called $\Gamma$-matrices $\Gamma_{I}$ which satisfy the Clifford relations $(I, J=1, \ldots, n)$ :

$$
\begin{aligned}
& \Gamma_{I}^{2}=\mathbb{I}, \quad \Gamma_{I} \Gamma_{J}+\Gamma_{J} \Gamma_{I}=0 \quad(I \neq J) ; \\
& \Uparrow \\
& \Gamma_{I} \Gamma_{J}+\Gamma_{J} \Gamma_{I}=2 \delta_{I J} \mathbb{I} .
\end{aligned}
$$

A set of $n$ matrices $\left\{\Gamma_{1}, \ldots, \Gamma_{n}\right\}$ of size $m$ will be called a Clifford set (of cardinality $n$ and size $m$ ). In (5.25) we will define specific $\Gamma$-matrices. 
Conversely, given a Clifford set $\left\{\Gamma_{1}, \ldots, \Gamma_{n}\right\} \subset M_{m}(\mathbb{R})$ the definition of the Clifford algebra shows that there is a unique homomorphism $C l(n, 0) \rightarrow M_{m}(\mathbb{R})$ sending $e_{I} \mapsto \Gamma_{I}$. Since the matrix algebras $M_{m}(\mathbb{K})$, with $\mathbb{K}=\mathbb{R}, \mathbb{C}, \mathbb{H}$ are simple (so any homomorphism $M_{m}(\mathbb{K}) \rightarrow M_{r}(\mathbb{R})$ is either injective or it is the zero homomorphism), it follows from the isomorphisms (5.5) (5.6) and (5.7) that a Clifford set with matrices of size 1, 2, 4, 8, 16 can have cardinality at most $1,2,3,5,9$, respectively.

Furthermore, if $\left\{\Gamma_{1}, \ldots, \Gamma_{n}\right\}$ is a Clifford set, one easily checks that

$$
\left(\sum_{I=1}^{n} x^{I} \Gamma_{I}\right)^{2}=\left(\sum_{I=1}^{n}\left(x^{I}\right)^{2}\right) \mathbb{I}_{2^{g}} .
$$

\subsection{The product decomposition for $n \equiv 1 \bmod 4$}

We recall the product decomposition of the Euclidean Clifford algebras $C l(n, 0)$ in case $n \equiv 1 \bmod 4$. The key point are the following elements in such a Clifford algebra, where we use the notation from section 5.1:

$$
c:=e_{1} e_{2} \ldots e_{n}, \quad c_{+}:=(1+c) / 2, \quad c_{-}:=(1-c) / 2 \quad(\in C l(n, 0)) .
$$

Since $e_{I}^{2}=1$ and $e_{I} e_{J}=-e_{J} e_{I}$ if $I \neq J$, one easily verifies that

$$
c^{2}=e_{1} e_{2} \ldots e_{n} e_{1} e_{2} \ldots e_{n}=(-1)^{n-1} e_{1} e_{2} e_{3} \ldots e_{n-1} e_{n}^{2} e_{1} e_{2} e_{3} \ldots e_{n-1}=(-1)^{n(n-1) / 2},
$$

and that for any $I$ we have

$$
c e_{I}=e_{1} e_{2} \ldots e_{n} c=(-1)^{n-1} e_{I} e_{1} e_{2} \ldots e_{n}=(-1)^{n-1} e_{I} c .
$$

In particular, if $n \equiv 1 \bmod 2$ then we have $c e_{I}=e_{I} c$ which implies that $c x=x c$ for all $x \in C l(n)$ since the $e_{I}$ generate the Clifford algebra. Notice that then also the $c_{ \pm}$are central elements, $x c_{ \pm}=c_{ \pm} x$ for all $x \in C l(n, 0)$. If moreover $n \equiv 1 \bmod 4$ then we also have that $c^{2}=1$.

Now we assume that $n \equiv 1 \bmod 4$. Then we find that

$$
c_{ \pm}^{2}=(1 \pm c)(1 \pm c) / 4=\left(1 \pm 2 c+c^{2}\right) / 4=(1 \pm c) / 2=c_{ \pm}
$$

and recall that the $c_{ \pm}$are central:

$$
c_{+} c_{-}=c_{-} c_{+}=\left(c^{2}-1\right) / 4=0,
$$

so the $c_{ \pm}$are central idempotents in $C l(n, 0)$. This causes the algebra to split into two components as follows. For $x \in C l(n, 0)$ let

$$
x=x(1+c) / 2+x(1-c) / 2=x_{+}+x_{-},
$$

and thus the vector space $C l(n, 0)$ splits as

$$
C l(n, 0)=C l(n, 0)_{+} \oplus C l(n, 0)_{-}, \quad C l(n, 0)_{ \pm}:=\left\{x c_{ \pm}=c_{ \pm} x: x \in C l(n, 0)\right\},
$$


the sum is direct since if $z=x c_{+}=y c_{-}$then $z c_{+}=y c_{-} c_{+}=0$ and $z_{-}=x c_{+} c_{-}=0$ hence $z=z c_{+}+z c_{-}=0$. The subspaces $C l(n, 0)_{ \pm}$are actually subalgebras: if $u, v \in C l(n, 0)_{ \pm}$ then also $u v \in C l(n, 0)_{ \pm}$since we can write $u=x c_{ \pm}, v=y c_{ \pm}$and thus

$$
u v=\left(x c_{ \pm}\right)\left(y c_{ \pm}\right)=x y c_{ \pm}^{2}=x y c_{ \pm} \quad \in C l(n, 0)_{ \pm} .
$$

In particular, given a matrix representation (an algebra homomorphism) $\phi: C l(n, 0) \rightarrow$ $M_{m}(\mathbb{R})$ defined by $\phi\left(e_{I}\right)=\Gamma_{I}$, the element $c$ maps to $\Gamma_{1} \Gamma_{2} \ldots \Gamma_{n}$ and $c^{2}=1$ maps to $\mathbb{I}_{m}$. If the representation is irreducible, then by Schur's lemma any element, like $\phi(c)$, that commutes with all $\phi(x), x \in C l(n, 0)$, is a scalar multiple of the identity and since $c^{2}=1$ it follows that $\phi(c)= \pm \mathbb{I}_{m}$. In case $c=\mathbb{I}_{m}$ one finds $\phi\left(c_{+}\right)=\mathbb{I}_{m}$ and $\phi\left(c_{-}\right)=0$, so that $C l(n, 0)_{-}=\operatorname{ker}(\phi)$, but in case $c=-\mathbb{I}_{m}$ one finds $\phi\left(c_{-}\right)=\mathbb{I}_{m}$ and $\phi\left(c_{+}\right)=0$, so that $C l(n, 0)_{+}=\operatorname{ker}(\phi)$.

Notice also that changing the sign of all $\Gamma_{I}, I=1, \ldots, n$, one obtains again a representation $\phi_{-}: C l(n, 0) \rightarrow M_{m}(\mathbb{R})$ (with $\phi_{-}\left(e_{I}\right)=-\Gamma_{I}$ ) but now, since $n$ is odd, we have $\phi_{-}(c)=-\phi(c)$. So, as is well-known, the representations $\phi$ and $\phi_{-}$are not equivalent. More generally, changing the sign of an odd number of the $\Gamma_{I}$ defines a representation which is not equivalent to $\phi$ since it changes the sign of $\phi(c)$.

\subsection{Quadratic forms and $\Gamma$-matrices}

Recall the definition of the four $2 \times 2$ (Pauli) matrices:

$$
\begin{aligned}
& \gamma_{00}:=\mathbb{I}_{2}=\left(\begin{array}{ll}
1 & 0 \\
0 & 1
\end{array}\right), \quad \gamma_{10}:=\sigma_{1}=\left(\begin{array}{ll}
0 & 1 \\
1 & 0
\end{array}\right), \\
& \gamma_{01}=\sigma_{3}=\left(\begin{array}{cc}
1 & 0 \\
0 & -1
\end{array}\right), \quad \gamma_{11}=i \sigma_{2}=\left(\begin{array}{rr}
0 & 1 \\
-1 & 0
\end{array}\right) .
\end{aligned}
$$

The notation is chosen such that (we recall that $i, j, k, l=0,1$ )

$$
\gamma_{i j} \gamma_{k l}= \pm \gamma_{(i+k)(j+l)}
$$

where the indices are summed modulo 2. Notice that

$$
\gamma_{i j}^{2}=(-1)^{i j} \mathbb{I}_{2}, \quad \gamma_{i j} \gamma_{k l}=(-1)^{i l+j k} \gamma_{k l} \gamma_{i j}, \quad{ }^{T} \gamma_{i j}=(-1)^{i j} \gamma_{i j}
$$

Recall that the tensor product of the square matrices $M=\left(M_{i j}\right)$ and $N=\left(N_{k l}\right)$, of size $m \times m$ and $n \times n$ respectively, is the matrix of size $n m \times n m$ given by the (block) matrix

$$
M \otimes N:=\left(\begin{array}{ccc}
M N_{11} & \ldots & M N_{1 n} \\
\vdots & \vdots & \vdots \\
M N_{n 1} & \ldots & M N_{n n}
\end{array}\right) .
$$

By a $\Gamma$-matrix (of size $2^{g} \times 2^{g}$, and characteristic $\left[\begin{array}{l}i_{1} \ldots i_{g} \\ j_{1} \ldots j_{g}\end{array}\right]$ ), in this paper we intend the following tensor product:

$$
\Gamma \equiv \Gamma\left[\begin{array}{c}
i_{1} \ldots i_{g} \\
j_{1} \ldots j_{g}
\end{array}\right]:=\gamma_{i_{1} j_{1}} \otimes \ldots \otimes \gamma_{i_{g} j_{g}}
$$


Such a $\Gamma$-matrix is symmetric iff the sum of the products $i_{k} j_{k}$ is zero modulo 2 :

$$
\begin{aligned}
{ }^{T} \Gamma & ={ }^{T}\left(\gamma_{i_{1} j_{1}} \otimes \ldots \otimes \gamma_{i_{g} j_{g}}\right)=\left({ }^{T} \gamma_{i_{1} j_{1}}\right) \otimes \ldots \otimes\left({ }^{T} \gamma_{i_{g} j_{g}}\right) \\
& =(-1)^{\sum_{a=1}^{g} i_{a} j_{a}}\left(\gamma_{i_{1} j_{1}} \otimes \ldots \otimes \gamma_{i_{g} j_{g}}\right)=(-1)^{\sum_{a=1}^{g} i_{a j}{ }^{i_{a}}} \Gamma .
\end{aligned}
$$

The quadratic form (with characteristic $\left[\begin{array}{l}i_{1} \ldots i_{g} \\ j_{1} \ldots j_{g}\end{array}\right]$ ) in $y^{1}, \ldots, y^{2^{g}}$ associated to the $\Gamma$-matrix (5.25) is defined as

$$
Q\left[\begin{array}{l}
i_{1} \ldots i_{g} \\
j_{1} \ldots j_{g}
\end{array}\right](y):={ }^{T} y\left(\gamma_{i_{1} j_{1}} \otimes \ldots \otimes \gamma_{i_{g} j_{g}}\right) y .
$$

If $\sum_{a=1}^{g} i_{a} j_{a}=0$ modulo 2 , the parity of the characteristic is said to be even, and then (5.26) shows that the $\Gamma$-matrix is symmetric. The (anti)symmetric $\Gamma$ matrices of size $2^{g}$ are a basis of the vector space of (skew)symmetric matrices of size $2^{g}$.

\subsection{Examples of the quadratic forms}

In case $g=1$ there are 3 symmetric $\Gamma$-matrices, $\gamma_{00}, \gamma_{01}, \gamma_{10}$ and the corresponding quadratic forms are respectively:

$$
Q\left[\begin{array}{l}
0 \\
0
\end{array}\right]:=\left(y^{1}\right)^{2}+\left(y^{2}\right)^{2}, \quad Q\left[\begin{array}{l}
0 \\
1
\end{array}\right]:=\left(y^{1}\right)^{2}-\left(y^{2}\right)^{2}, \quad Q\left[\begin{array}{l}
1 \\
0
\end{array}\right]:=2 y^{1} y^{2} .
$$

For $g=2$ there are 10 symmetric (and 6 antisymmetric) $\Gamma$ matrices, here are some examples of the corresponding quadratic forms with $\Gamma$-matrices $\sigma_{1} \otimes \sigma_{1}$ and $\sigma_{1} \otimes \sigma_{3}$ respectively:

$$
Q\left[\begin{array}{l}
11 \\
00
\end{array}\right]:=2\left(y^{1} y^{4}+y^{2} y^{3}\right), \quad Q\left[\begin{array}{l}
01 \\
10
\end{array}\right]:=2\left(y^{1} y^{2}-y^{3} y^{4}\right) .
$$

In case $g=3$ there are 36 symmetric and 28 antisymmetric $\Gamma$-matrices and for example the quadratic form corresponding to $\gamma_{10} \otimes \gamma_{11} \otimes \gamma_{11}$ is

$$
Q\left[\begin{array}{l}
111 \\
110
\end{array}\right]:=2\left(y^{1} y^{8}+y^{2} y^{7}-y^{3} y^{6}-y^{4} y^{5}\right) .
$$

\subsection{Clifford sets of $\Gamma$-matrices}

One easily verifies:

$$
\begin{aligned}
\Gamma\left[\begin{array}{c}
i_{1} \ldots i_{g} \\
j_{1} \ldots j_{g}
\end{array}\right] \Gamma\left[\begin{array}{l}
k_{1} \ldots k_{g} \\
l_{1} \ldots l_{g}
\end{array}\right] & =\left(\gamma_{i_{1} j_{1}} \otimes \ldots \otimes \gamma_{i_{g} j_{g}}\right)\left(\gamma_{k_{1} l_{1}} \otimes \ldots \otimes \gamma_{k_{g} l_{g}}\right) \\
& =(-1)^{\sum_{a=1}^{g} i_{a} l_{a}+j_{a} k_{a}}\left(\gamma_{k_{1} l_{1}} \otimes \ldots \otimes \gamma_{k_{g} l_{g}}\right)\left(\gamma_{i_{1} j_{1}} \otimes \ldots \otimes \gamma_{i_{g} j_{g}}\right) \\
& =(-1)^{\sum_{a=1}^{g} i_{a} l_{a}+j_{a} k_{a}} \Gamma\left[\begin{array}{l}
k_{1} \ldots k_{g} \\
l_{1} \ldots l_{g}
\end{array}\right] \Gamma\left[\begin{array}{l}
i_{1} \ldots i_{g} \\
j_{1} \ldots j_{g}
\end{array}\right] \\
\left(\Gamma\left[\begin{array}{l}
i_{1} \ldots i_{g} \\
j_{1} \ldots j_{g}
\end{array}\right]\right)^{2} & =\left(\gamma_{i_{1} j_{1}} \otimes \ldots \otimes \gamma_{i_{g} j_{g}}\right)^{2}=(-1)^{\sum_{a=1}^{g} i_{a} j_{a}} \mathbb{I}_{2^{g}} .
\end{aligned}
$$

Note that (5.26) and (5.32) show that if a $\Gamma$-matrix $\Gamma\left[\begin{array}{l}i \\ j\end{array}\right]$ is symmetric then $\Gamma\left[\begin{array}{l}i \\ j\end{array}\right]^{2}=\mathbb{I}_{2^{g}}$. Moreover, from (5.31) it follows that two distinct symmetric $\Gamma$-matrices $\Gamma\left[\begin{array}{l}i \\ j\end{array}\right]$ and $\Gamma\left[\begin{array}{l}k \\ l\end{array}\right]$ anti-commute iff the characteristic $\left[\begin{array}{c}i+k \\ j+l\end{array}\right]$ is odd, that is $\sum_{a=1}^{g}\left(i_{a}+k_{a}\right)\left(j_{a}+l_{a}\right) \equiv 1 \bmod 2$, (use that the symmetry implies $\sum_{a=1}^{g} i_{a} j_{a} \equiv \sum_{a=1}^{g} k_{a} l_{a} \equiv 0 \bmod 2$ ).

Thus a set of $\Gamma$-matrices $\left\{\Gamma\left[\begin{array}{l}i^{1} \\ j^{1}\end{array}\right], \ldots, \Gamma\left[i_{j^{n}}^{i n}\right]\right\}$ is a Clifford set iff all characteristics $\left[\begin{array}{l}i^{1} \\ j^{1}\end{array}\right], \ldots,\left[\begin{array}{l}i^{n} \\ j^{n}\end{array}\right]$ are even and the sum of any two distinct characteristics is odd. 


\subsection{Quadratic identities between quadratic forms}

Whereas the quadratic forms $Q\left[\begin{array}{l}i \\ j\end{array}\right]$ (with even characteristics of length $g$ ) are a basis of the vector space of all quadratic forms in $m=2^{g}$ variables, their squares, homogeneous polynomials of degree four, are not linear independent. The most basic example, known as Jacobi's identity, is:

$$
-Q\left[\begin{array}{l}
0 \\
0
\end{array}\right]^{2}+Q\left[\begin{array}{l}
0 \\
1
\end{array}\right]^{2}+Q\left[\begin{array}{l}
1 \\
0
\end{array}\right]^{2}=0,
$$

which is easily verified since, by definition,

$$
Q\left[\begin{array}{l}
0 \\
0
\end{array}\right]:=\left(y^{1}\right)^{2}+\left(y^{2}\right)^{2}, \quad Q\left[\begin{array}{l}
0 \\
1
\end{array}\right]:=\left(y^{1}\right)^{2}-\left(y^{2}\right)^{2}, \quad Q\left[\begin{array}{l}
0 \\
1
\end{array}\right]:=2 y^{1} y^{2},
$$

and thus we indeed have the identity

$$
\left(\left(y^{1}\right)^{2}+\left(y^{2}\right)^{2}\right)^{2}=\left(\left(y^{1}\right)^{2}-\left(y^{2}\right)^{2}\right)^{2}+\left(2 y^{1} y^{2}\right)^{2} .
$$

For $g=2,3,4$ there are similar identities between 4,6,10 respectively such quadrics, see (8.61), (8.37), (8.5) respectively. A remarkable fact, and crucial for the invertibility results in this paper, is that in all these four cases the Gamma-matrices of the quadrics are $\mathbb{I}_{m}$ and the remaining $\Gamma_{I}$ form a (maximal) Clifford set. Actually, these identities are classical identities between theta functions. The case $g=1$ was known to Jacobi, and the other cases were already known to Max Noether, see [110, p.332, p.334].

Unfortunately, for $g>4$ it seems that the squares of the quadrics defined by $\mathbb{I}_{m}$ and a maximal Clifford set are no longer linearly dependent.

\subsection{Heisenberg groups}

The $\Gamma$-matrices generate a finite (non-Abelian) subgroup of $\operatorname{GL}\left(2^{g}, \mathbb{C}\right)$, which is called a Heisenberg group (cfr. e.g. [40]) or a Clifford group. The subgroup has order $2 \cdot 2^{2 g}$ and each element is of the form $\pm \Gamma$ where $\Gamma$ is one of the $2^{2 g} \Gamma$-matrices. See [40, appendix A] for the quadratic forms $Q_{m}=Q\left[{ }_{\epsilon^{\prime}}^{\epsilon}\right]$. The quadratic relations among the $Q_{m}$ 's that we discussed can also be found in A.3 of [40], where they are discussed in the context of Hopf maps.

\section{BPS entropy and attractors in $L(q, P)$ models}

In the present paper we focus on BPS systems related to models $L(q, P, \dot{P})$ of homogeneous very special geometry [53]: we will thus only consider the case of a Lorentzian quadratic form in the $x^{i}$ and a related Clifford set of $\Gamma$-matrices. In particular (see section 4.3), we consider $V$ (cfr. (4.56)) to have Lorentzian (mostly plus) signature and

$$
\operatorname{dim} V=q+2
$$

such that (cfr. (4.27))

$$
N=\operatorname{dim} V+1+\mathcal{D}_{\operatorname{dim} V-1} \cdot(P+\dot{P})
$$


For later convenience, in order to highlight the Lorentzian (mostly plus) signature, we also shift the labeling of the $q+2 I$-indices from $1, \ldots, q+2$ to $0,1, \ldots, q+1$; moreover, the matrix $\Gamma_{0}$ will be nothing but the identity matrix of size $m=\mathcal{D}_{q+1} \cdot(P+\dot{P})$.

Physically, the $L(q, P, \dot{P})$ models of homogeneous very special geometry determine the scalar manifolds (i.e., the target spaces of scalar fields) in ungauged $\mathcal{N}=2$ Maxwell-Einstein supergravity theory coupled to vector multiplets in three, four or five Lorentzian space-time dimensions (the corresponding spaces are quaternionic, Kähler and real, respectively).

Since the closed form expression of the Bekenstein-Hawking entropy (2.2) as well as of the attractor values of scalar fields (2.13) of extremal BPS black holes are already known for symmetric spaces (see e.g. [62]), in the present paper we will focus on homogeneous non-symmetric spaces.

In the present section, we will start from a Clifford set, and we will define a certain cubic form; then, we will show that under certain conditions its gradient map is invertible; consequently, the corresponding BPS system (2.6) can be explicitly solved by (6.53), thus allowing for a novel, closed form expression of the Bekenstein-Hawking entropy (2.2) as well as of the attractor values of scalar fields (2.13) of extremal BPS black holes, respectively given by (6.66) and (6.73)-(6.75) below.

After such a general treatment, we will consider some classes of models, namely:

- $L(q, 1)$ with $q=1, \ldots, 8$ (see section 8 );

- $L(q, 2)$ with $q=1,2,3$ (see section 9 );

- $L(q, P), q=1,2,3, P \geqslant 3$ (see section 10), with explicit emphasis given to the models $L(1, P)$ with $P \geqslant 2$ given in section 10.7 ;

- $L(4,1,1)$ (see section 12); in the present paper, this is the unique model having a non-vanishing $\dot{P}$ (namely, with $P=\dot{P}=1$ ).

In particular, the models $L(1,2)$ and $L(1,3)$ will be analyzed in full detail in sections 9.5 and 10.6, respectively, and their analysis will be explicitly generalized to the class of models $L(1, P)$ with $P \geqslant 2$ in section 10.7. In this respect, such sections extend the treatment given $^{21}$ in section 4 of [122], by providing explicit expressions for the BPS black hole entropy as well as for the BPS attractors in such an infinite class of non-symmetric (homogeneous) models of $\mathcal{N}=2, D=4$ supergravity with cubic prepotential.

It is also here worth anticipating that similar results can be obtained also starting with quadratic forms with different signatures; for instance, we will briefly deal with a ten-dimensional quadratic form with Kleinian signature (i.e., with $\epsilon_{1}=\ldots=\epsilon_{5}=1=$ $\left.-\epsilon_{6}=\ldots=-\epsilon_{10}\right)$ in section 13.

\footnotetext{
${ }^{21}$ It would be interesting to investigate the geometric aspects of the examples of non-homogeneous very special geometry discussed in section 4 of [122] (cfr. refs. therein, as well); we leave this task for further future work.
} 


\subsection{The cubic form}

Let $\left\{\Gamma_{1}, \ldots, \Gamma_{q+1}\right\}$ be a Clifford set of symmetric $\Gamma$-matrices of size $m \times m$, with $m=\mathcal{D}_{q+1}$ as in (4.26) and section 5.6. Let $\Gamma_{0}:=\mathbb{I}_{m}$.

We define a Lorentzian quadratic form $q$ in $q+2$ variables $x^{0}, \ldots, x^{q+1}$ and $q+2$ quadratic forms $Q_{I}, I=0, \ldots, q+1$, in $m=\mathcal{D}_{q+1}$ variables $y^{1}, \ldots, y^{m}:$ (cf. eq. (4.58) above):

$$
\begin{aligned}
q(x) & :=-\left(x^{0}\right)^{2}+\left(x^{1}\right)^{2}+\ldots+\left(x^{q+1}\right)^{2} ; \\
Q_{I}(y) & :={ }^{T} y \Gamma_{I} y .
\end{aligned}
$$

Using the these quadratic forms, we define the cubic form in $1+(q+2)+m$ variables $s$, $x^{0}, \ldots, x^{q+1}, y^{1}, \ldots, y^{m}$ :

$$
\mathcal{V}(s, x, y):=s q(x)+x^{0} Q_{0}(y)+\ldots+x^{q+1} Q_{q+1}(y) .
$$

\subsection{The invertibility condition}

Given the Clifford set $\left\{\Gamma_{1}, \ldots, \Gamma_{q+1}\right\}$, in order to invert the gradient map of $\mathcal{V}$, we need the existence of further symmetric $m \times m$ matrices $\Omega_{1}, \ldots, \Omega_{r}$, which anti-commute with the matrices in the Clifford set:

$$
\Gamma_{I} \Omega_{K}=-\Omega_{K} \Gamma_{I} \quad(I=1, \ldots, q+1, \quad K=1, \ldots, r) .
$$

Moreover, if we denote the associated quadratic forms defined by these $\Omega_{K}$ by

$$
R_{K}(y):={ }^{T} y \Omega_{K} y, \quad K=1, \ldots, r,
$$

then the following Lorentzian quadratic identity should hold:

$$
-Q_{0}(y)^{2}+\sum_{I=1}^{q+1} Q_{I}(y)^{2}+\sum_{K=1}^{r} R_{K}(y)^{2}=0 .
$$

If auxiliary matrices $\Omega_{K}$ with all these properties exist, then the gradient map (with $I=0, \ldots, q+1$, and $\alpha=1, \ldots, m)$

$$
\begin{aligned}
& \nabla_{\mathcal{V}}: \mathbb{R}^{q+3+m} \longrightarrow \mathbb{R}^{q+3+m} ; \\
& \nabla_{\mathcal{V}}={ }^{T}\left(\mathcal{V}_{s}, \ldots, \mathcal{V}_{I}, \ldots, \mathcal{V}_{\alpha}, \ldots\right), \\
& \mathcal{V}_{s}:=\frac{\partial \mathcal{V}}{\partial s}, \quad \mathcal{V}_{I}:=\frac{\partial \mathcal{V}}{\partial x^{I}}, \quad \mathcal{V}_{\alpha}:=\frac{\partial \mathcal{V}}{\partial y^{\alpha}},
\end{aligned}
$$

is invertible, with (birational) inverse given by polynomials of degree 2 if $r=0$ and of degree 4 if $r>0$. An explicit expression of the birational inverse map of the gradient map $\nabla \mathcal{V}$ will be given in section 6.4. This results in a closed form expression of the solution to the BPS system (2.6), given in section 6.5. In turn, in section 6.6 this will allow for a closed form expression of Bekenstein-Hawking entropy (2.2) as well as of the attractor values of scalar fields (2.13) of extremal BPS black holes in the homogeneous non-symmetric very special geometry characterizing the corresponding model of ungauged $\mathcal{N}=2$ Maxwell-Einstein supergravity theory coupled to vector multiplets in four space-time dimensions. 


\subsection{Remarks}

Notice that only in the case that we do have a Lorentzian identity $-Q_{0}(y)^{2}+\sum_{I=1}^{q+1} Q_{I}(y)^{2}=$ 0 , there is no need for the extra $\Omega_{K}$ 's (and one can then take $r=0$ ).

As it will be seen in section 6.7, this is a quite special case, corresponding to some symmetric very special spaces, and to the very rich geometry related to simple cubic Jordan algebras $[115,116]$. In the present investigation, since we want to solve the BPS system (2.6) and study the expression of the BPS black hole entropy (2.2) in cases not treated in literature, we will be interested in some classes of homogeneous non-symmetric spaces which all have $r>0$.

It seems rather restrictive (and mysterious) to request the existence of the (Lorentzian) quadratic identity (6.8), but it is crucial for us in order to show the existence of a (birational) inverse map of the gradient map of the corresponding cubic form $\mathcal{V}$, and thus to provide a closed form expression of the solution to the associated BPS system (2.6).

It is here worth remarking that we will not impose any condition on products involving only $\Omega$-matrices; in particular, for $P \geqslant 3$, we will consider symmetric $m \times m$ matrices $\Omega_{j}$ 's that are not invertible (i.e. whose rank is less than $m$ ), so they cannot be $\Gamma$-matrices (see section 10).

We conclude with some remarks on the inverse of the gradient map $\nabla_{\mathcal{V}}$. This gradient map is given by the partial derivatives of $\mathcal{V}$ which are homogeneous polynomials of degree two in $\xi:=(s, x, y)$. Therefore $\nabla \mathcal{V}(\xi)=\nabla \mathcal{V}(-\xi)$ and this implies that the inverse image of the image of any non-zero point contains at least two points which differ by a sign.

The inverse map $\nabla \mathcal{V}^{-1}$ will be given by homogeneous polynomials of degree four in general, and thus the composition $\nabla \mathcal{V}^{-1} \circ \nabla \mathcal{V}$ has coordinate functions that are homogeneous of degree eight. Therefore it cannot be the identity map (since the identity map has coordinate functions that are homogeneous of degree one), but one has:

$$
\left((\nabla \mathcal{V})^{-1} \circ \nabla \mathcal{V}\right)(\xi)=f(\xi) \xi, \quad f(\xi)=4 q(\xi)^{2} \mathcal{V}(\xi)
$$

(cfr. (6.13)), with $f(\xi)$ homogeneous of degree $2 \cdot 2+3=7$. In particular, for the points $\xi$ with $f(\xi)=0$ the inverse of the gradient map does not provide useful information.

All this should not be surprising, in fact in the simple cubic Jordan algebra models the gradient map is given by $M \mapsto M^{\sharp}$ where $M^{\sharp}$ is the adjoint of a $3 \times 3$ matrix and one has $M^{\sharp}=(-M)^{\sharp}$; the inverse of the adjoint map is then $M^{\sharp} \mapsto\left(M^{\sharp}\right)^{\sharp}$ which is $M$, up to a homogeneous polynomial of degree three which is the determinant of $M:\left(M^{\sharp}\right)^{\sharp}=\operatorname{det}(M) M$ (and $\operatorname{det}(M)$ is basically the cubic form $\mathcal{V}$ of the corresponding $L(q, 1)$ model), see also section 8.2.7.

\subsection{Explicit inversion of $\nabla_{\mathcal{V}}$}

When $r>0$, the inverse of the gradient map $\nabla_{\mathcal{V}}$ is given as a composition of two maps, namely:

$$
\begin{aligned}
& \mathbb{R}_{(s, x, y)}^{q+3+m} \stackrel{\nabla_{\mathcal{V}}}{\longrightarrow} \mathbb{R}_{z}^{q+3+m} \stackrel{\alpha}{\longrightarrow} \mathbb{R}_{(t, u, v, w)}^{q+3+m+r} \stackrel{\mu}{\longrightarrow} \mathbb{R}_{(s, x, y)}^{q+3+m}, \\
& \text { such that } \mu \circ \alpha \circ \nabla_{\mathcal{V}}=4 q(x)^{2} \mathcal{V}(s, x, y) \mathbb{I}_{q+3+m} .
\end{aligned}
$$


The map $\alpha$, which has $q+3+m+r$ components that are homogeneous polynomials of degree 2 in the variables $z_{1}, \ldots, z_{q+3+m}$, is given by

$$
\alpha\left(z_{1}, \ldots, z_{q+3+m}\right):={ }^{T}\left(z_{1}^{2}, z_{1} z_{2}, \ldots, z_{1} z_{q+3+m}, R_{1}(z), \ldots, R_{r}(z)\right),
$$

where the $r$ quadratic forms $R_{K}(z)$ 's depend only on the last $m$ variables $z_{q+3+1}, \ldots, z_{q+3+m}$ :

$$
R_{K}(z):=R_{K}\left(z_{q+3+1}, \ldots, z_{q+3+m}\right) .
$$

The composition $\alpha \circ \nabla_{\mathcal{V}}$ will be explicitly computed in section 7.2 below, and it is given by

$$
\alpha \circ \nabla \mathcal{V}(s, x, y):=\alpha(\nabla \mathcal{V}(s, x, y))=q(x)^{T}\left(\nabla \mathcal{V}(s, x, y),-4 R_{1}(y), \ldots,-4 R_{r}(y)\right) .
$$

Next, the map $\mu$, which has $q+3+m$ components that are homogeneous polynomials of degree 2 in the variables $t, u_{0}, \ldots, u_{q+1}, v_{1}, \ldots, v_{m}, w_{1} \ldots, w_{r}$, is given by

$$
\mu(t, u, v, w):=\left(\begin{array}{c}
q(u)+\frac{1}{16} \sum_{K=1}^{r} w_{K}^{2} \\
-2 t u_{0}+\frac{1}{2} Q_{0}(v) \\
2 t u_{1}+\frac{1}{2} Q_{1}(v) \\
\vdots \\
2 t u_{q+1}+\frac{1}{2} Q_{q+1}(v) \\
\left(\sum_{I=0}^{q+1} u_{I} \Gamma_{I}\right) v-\frac{1}{4}\left(\sum_{K=1}^{r} w_{K} \Omega_{K}\right) v
\end{array}\right),
$$

where the definition of the last $m$ components involves all the $q+2$ symmetric $\Gamma$-matrices $\left\{\Gamma_{I}\right\}$ as well as the $r$ auxiliary symmetric matrices $\left\{\Omega_{K}\right\}$, required in the invertibility condition enounced in section 6.2 ; all these matrices have size $m \times m$.

Recalling the relabelling (4.41), we will verify in section 7 that the composition of maps $\mu \circ \alpha \circ \nabla_{\mathcal{V}}$ is given by (cfr. (6.5))

$$
\begin{gathered}
\left(\begin{array}{c}
(\mu \circ \alpha)^{s}\left(\nabla_{\mathcal{V}}(s, x, y)\right) \\
(\mu \circ \alpha)^{I}\left(\nabla_{\mathcal{V}}(s, x, y)\right) \\
(\mu \circ \alpha)^{\alpha}\left(\nabla_{\mathcal{V}}(s, x, y)\right)
\end{array}\right)=4 q^{2}(x) \mathcal{V}(s, x, y)\left(\begin{array}{c}
s \\
x^{I} \\
y^{\alpha}
\end{array}\right) \\
\Uparrow \\
\qquad \circ \alpha \circ \nabla_{\mathcal{V}}(\xi)=4 q^{2}(x) \mathcal{V}(\xi) \xi .
\end{gathered}
$$

As we will see below, in complete models (see section 6.7) $r=0$ by definition, and one can omit the map $\alpha$ (because it becomes proportional to the identity map), and then (6.19) reduces to

$$
\mu \circ \nabla \mathcal{V}(\xi)=4 \mathcal{V}(\xi) \xi
$$

The identity (6.19) implies that the composed map

$$
\mathbb{R}_{z}^{q+3+m} \stackrel{\mu \circ \alpha}{\longrightarrow} \mathbb{R}_{(s, x, y)}^{q+3+m}
$$


can be regarded as the inverse map of the gradient map $\nabla_{\mathcal{V}}$. In order to determine the general form of the map $\mu \circ \alpha$ (6.21), the map $\mu$ (6.17) must be evaluated on the image of the map $\alpha(6.14)$ :

$$
(\mu \circ \alpha)\left(z_{1}, \ldots, z_{q+3+m}\right):=\mu\left(\alpha\left(z_{1}, \ldots, z_{q+3+m}\right)\right) .
$$

The replacement of the variables $t, u_{0}, \ldots, u_{q+1}, v_{1}, \ldots, v_{m}, w_{1} \ldots, w_{r}$ with the corresponding components (homogeneous polynomials in the variables $z_{1}, \ldots, z_{q+3+m}$ ) in the image of $\alpha$ (6.14) reads as follows $(I=0,1, \ldots, q+1, \alpha=1, \ldots, m, K=1, \ldots, r)$ :

$$
\left\{\begin{array}{l}
t \rightarrow z_{1}^{2} \\
u_{I} \rightarrow z_{1} z_{I+2} \\
v_{\alpha} \rightarrow z_{1} z_{q+3+\alpha} \\
w_{K} \rightarrow R_{K}(z) \stackrel{(6.15)}{=} R_{K}\left(z_{q+3+1}, \ldots, z_{q+3+m}\right) .
\end{array}\right.
$$

By defining the $m \times 1$ vector

$$
\hat{z}:={ }^{T}\left(z_{q+4}, \ldots, z_{q+3+m}\right)
$$

one can easily compute:

$$
(\mu \circ \alpha)\left(z_{1}, \ldots, z_{q+3+m}\right):=\left(\begin{array}{c}
q\left(u_{I} \rightarrow z_{1} z_{I+2}\right)+\frac{1}{16} \sum_{K=1}^{r} R_{K}^{2}(\hat{z}) \\
-2 z_{1}^{3} z_{2}+\frac{1}{2} Q_{0}\left(v \rightarrow z_{1} \hat{z}\right) \\
2 z_{1}^{3} z_{3}+\frac{1}{2} Q_{1}\left(v \rightarrow z_{1} \hat{z}\right) \\
\vdots \\
2 z_{1}^{3} z_{q+3}+\frac{1}{2} Q_{q+1}\left(v \rightarrow z_{1} \hat{z}\right) \\
z_{1}^{2}\left(\sum_{I=0}^{q+1} z_{I+2} \Gamma_{I}\right) \hat{z}-\frac{1}{4} z_{1}\left(\sum_{K=1}^{r} R_{K}(\hat{z}) \Omega_{K}\right) \hat{z}
\end{array}\right),
$$

where the last line of the r.h.s. contains the product of the $m \times m$ matrices $\Gamma_{I}$ and $\Omega_{K}$ with the $m \times 1$ vector $\hat{z}$ (6.24). From the definitions (6.3) and (6.7), one computes

$$
\begin{aligned}
& q\left(u_{I} \rightarrow z_{1} z_{I+2}\right)=z_{1}^{2}\left(-z_{2}^{2}+z_{3}^{2}+\ldots+z_{q+3}^{2}\right) ; \\
& Q_{0}\left(v \rightarrow z_{1} \hat{z}\right)=z_{1}^{2} T_{\hat{z}} \Gamma_{0} \hat{z}=z_{1}^{2} \hat{z}_{m} \hat{z}=z_{1}^{2}\left(z_{q+4}^{2}+\ldots+z_{q+3+m}^{2}\right) ; \\
& Q_{1}\left(v \rightarrow z_{1} \hat{z}\right)=z_{1}^{2} T_{\hat{z}} \Gamma_{1} \hat{z} ; \\
& \vdots \\
& Q_{q+1}\left(v \rightarrow z_{1} \hat{z}\right)=z_{1}^{2}{ }^{T} \hat{z} \Gamma_{q+1} \hat{z},
\end{aligned}
$$


and therefore (6.25) can be further elaborated as follows:

$$
(\mu \circ \alpha)\left(z_{1}, \ldots, z_{q+3+m}\right):=\left(\begin{array}{c}
z_{1}^{2}\left(-z_{2}^{2}+z_{3}^{2}+\ldots+z_{q+3}^{2}\right)+\frac{1}{16} \sum_{K=1}^{r} R_{K}^{2}(\hat{z}) \\
-2 z_{1}^{3} z_{2}+\frac{1}{2} z_{1}^{2}\left(z_{q+4}^{2}+\ldots+z_{q+3+m}^{2}\right) \\
2 z_{1}^{3} z_{3}+\frac{1}{2} z_{1}^{2} T_{\hat{z}} \Gamma_{1} \hat{z} \\
\vdots \\
2 z_{1}^{3} z_{q+3}+\frac{1}{2} z_{1}^{2} T_{\hat{z} \Gamma_{q+1} \hat{z}} \\
z_{1}^{2}\left(\sum_{I=0}^{q+1} z_{I+2} \Gamma_{I}\right) \hat{z}-\frac{1}{4} z_{1}\left(\sum_{K=1}^{r} R_{K}(\hat{z}) \Omega_{K}\right) \hat{z}
\end{array}\right),
$$

where (by recalling (6.7) and (6.15))

$$
R_{K}(\hat{z})={ }^{T} \hat{z} \Omega_{K} \hat{z} .
$$

Each of the $q+3+m$ components of this composed map is given by an homogeneous polynomial of degree 4 in the $q+3+m$ variables $z_{1}, \ldots, z_{q+3+m}$. As it is evident, the explicit form of such polynomials depends on $q+2$ (symmetric) $\Gamma$-matrices $\Gamma_{I}$ (such that $\Gamma_{0}=\mathbb{I}_{m}$ ) as well as on the $r$ symmetric auxiliary matrices $\Omega_{K}$ and the corresponding quadratic forms $R_{K}$ defined in (6.7) and in (6.15). Note that both the $\Gamma_{I}$ 's and the $\Omega_{K}$ 's, as well as the corresponding quadratic forms $Q_{I}$ 's $(6.4)$ and $R_{K}$ 's $(6.7)$, occur in the invertibility condition enounced in section 6.2 (cfr. eqs. (6.6) and (6.8), respectively), which is assumed to hold throughout the treatment of this section, as well as of the subsequent sections $6.5-6.7$ and in the whole section 7 .

\subsection{Solution of the BPS system}

From (6.18)-(6.19), by replacing $\nabla_{\mathcal{V}}$ with $3 \partial_{p} \Delta=3^{T}\left(\Delta_{s}, \Delta_{I}, \Delta_{\alpha}\right)$, one obtains

$$
\mathcal{V} \xi=\frac{9}{4} \frac{1}{\Delta_{s}^{2}}(\mu \circ \alpha)\left(\partial_{p} \Delta\right) .
$$

By defining

$$
\begin{aligned}
\nabla \mathcal{V}(\xi) \cdot \xi:= & \frac{\partial \mathcal{V}}{\partial s} s+\frac{\partial \mathcal{V}}{\partial x^{J}} x^{J}+\frac{\partial \mathcal{V}}{\partial y^{\beta}} y^{\beta} \\
\left(\partial_{p} \Delta\right) \cdot \xi:= & \Delta_{s} s+\Delta_{J} x^{J}+\Delta_{\beta} y^{\beta} \\
\left(\partial_{p} \Delta\right) \cdot(\mu \circ \alpha)\left(\partial_{p} \Delta\right):= & \Delta_{s}(\mu \circ \alpha)^{s}\left(\partial_{p} \Delta\right) \\
& +\Delta_{J}(\mu \circ \alpha)^{J}\left(\partial_{p} \Delta\right)+\Delta_{\beta}(\mu \circ \alpha)^{\beta}\left(\partial_{p} \Delta\right),
\end{aligned}
$$

and recalling the Euler formula (3.2), one can replace $\nabla_{\mathcal{V}}(\xi)$ with $3 \partial_{p} \Delta$ and use (6.29) in order to obtain

$$
3 \mathcal{V}=\nabla_{\mathcal{V}}(\xi) \cdot \xi=3\left(\partial_{p} \Delta\right) \cdot \xi=\frac{27}{4} \frac{1}{\mathcal{V} \Delta_{s}^{2}}\left(\partial_{p} \Delta\right) \cdot(\mu \circ \alpha)\left(\partial_{p} \Delta\right)
$$


leading to

$$
\begin{gathered}
\mathcal{V}^{2}=\frac{9}{4} \frac{1}{\Delta_{s}^{2}}\left(\partial_{p} \Delta\right) \cdot(\mu \circ \alpha)\left(\partial_{p} \Delta\right) \\
|\mathcal{V}|=\frac{3}{2\left|\Delta_{s}\right|} \sqrt{\left(\partial_{p} \Delta\right) \cdot(\mu \circ \alpha)\left(\partial_{p} \Delta\right)},
\end{gathered}
$$

which is well defined for

$$
\left(\partial_{p} \Delta\right) \cdot(\mu \circ \alpha)\left(\partial_{p} \Delta\right)>0 .
$$

Then, from (6.29) and (6.35), it follows that

$$
\xi= \pm \frac{9}{4} \frac{1}{|\mathcal{V}| \Delta_{s}^{2}}(\mu \circ \alpha)\left(\partial_{p} \Delta\right)= \pm \frac{3}{2\left|\Delta_{s}\right|} \frac{(\mu \circ \alpha)\left(\partial_{p} \Delta\right)}{\sqrt{\left(\partial_{p} \Delta\right) \cdot(\mu \circ \alpha)\left(\partial_{p} \Delta\right)}}
$$

is the general solution of the BPS system (2.6). However, we must also recall the condition (2.12), which in this case reads

$$
\left(\partial_{p} \Delta\right) \cdot \xi>0 \Leftrightarrow \mathcal{V}(\xi)>0 \Leftrightarrow \pm \frac{3}{2\left|\Delta_{s}\right|} \sqrt{\left(\partial_{p} \Delta\right) \cdot(\mu \circ \alpha)\left(\partial_{p} \Delta\right)}>0,
$$

which thus implies that only the branch "+" of (6.37) is consistent.

Summarising, at least in those homogeneous $d$-spaces $[53,56]$ in which the invertibility condition enounced in section 6.2 is satisfied, there exists a quartic homogeneous polynomial map $\left(i=1, \ldots, q+3+m\right.$, where - cfr. below $\left.(6.2)-m=(P+\dot{P}) \mathcal{D}_{q+1}\right)$

$$
\begin{array}{r}
\mu \circ \alpha:\left(\mathbb{R}^{q+3+m}\right)^{*} \rightarrow \mathbb{R}^{q+3+m} ; \\
(\mu \circ \alpha)^{i}(z):=(\mu \circ \alpha)^{i j k l m} z_{j} z_{k} z_{l} z_{m},
\end{array}
$$

namely (cfr. the index splitting ${ }^{22}(4.41)$ as well as the result (6.27)):

$$
\begin{aligned}
& i=s:(\mu \circ \alpha)^{s j k l m} z_{j} z_{k} z_{l} z_{m}:=z_{s}^{2}\left(-z_{2}^{2}+z_{3}^{2}+\ldots+z_{q+3}^{2}\right)+\frac{1}{16} \sum_{K=1}^{r} R_{K}^{2}(\hat{z}) \\
& i=I:(\mu \circ \alpha)^{I j k l m} z_{j} z_{k} z_{l} z_{m}:=\delta_{s}^{(j \mid} \delta_{s}^{k}(\mu \circ \alpha)^{I \mid l m)} z_{j} z_{k} z_{l} z_{m}=z_{s}^{2}(\mu \circ \alpha)^{I l m} z_{l} z_{m} \\
& i=\alpha:(\mu \circ \alpha)^{\alpha j k l m} z_{j} z_{k} z_{l} z_{m}:=\delta_{s}^{(j \mid}(\mu \circ \alpha)^{\alpha \mid k l m)} z_{j} z_{k} z_{l} z_{m}=z_{s}(\mu \circ \alpha)^{\alpha k l m} z_{k} z_{l} z_{m}
\end{aligned}
$$

where

$$
\begin{aligned}
(\mu \circ \alpha)^{I l m} z_{l} z_{m} & :=2 z_{s} \eta_{I I} z_{I+2}+\frac{1}{2}{ }^{T} \hat{z} \Gamma_{I} \hat{z} \\
& =\left\{\begin{array}{l}
I=0:(\mu \circ \alpha)^{0 l m} z_{l} z_{m}:=-2 z_{s} z_{2}+\frac{1}{2} \sum_{\alpha=1}^{m} z_{q+3+\alpha}^{2} ; \\
I=1, \ldots, q+1:(\mu \circ \alpha)^{I l m} z_{l} z_{m}:=2 z_{s} z_{I+2}+\frac{1}{2} T^{T} \hat{z} \Gamma_{I} \hat{z} ;
\end{array}\right. \\
(\mu \circ \alpha)^{\alpha k l m} z_{k} z_{l} z_{m}: & : z_{s}\left(\sum_{I=0}^{q+1} z_{I+2} \Gamma_{I}\right) \hat{z}-\frac{1}{4}\left(\sum_{K=1}^{r} R_{K}(\hat{z}) \Omega_{K}\right) \hat{z},
\end{aligned}
$$

\footnotetext{
${ }^{22}$ The first value of the index $i$ has been denoted with $s$ in the splitting (4.41), whereas it has been denoted with 1 in the result (6.27). Throughout the following treatment, we will assume $z_{1} \equiv z_{s}$.
} 
where $\eta_{I I}$ (no sum on the repeated index $I$ ) denotes the non-vanishing (diagonal) components of the $(q+2)$-dimensional (mostly plus) Lorentzian metric $\eta_{I J}$ introduced in (4.40).

Therefore, one can compute the quintic homogeneous polynomial (6.32) in $\partial_{p^{i}} \Delta \equiv \Delta_{i}$ to read

$$
\begin{aligned}
( & \left.\partial_{p} \Delta\right) \cdot(\mu \circ \alpha)\left(\partial_{p} \Delta\right):=(\mu \circ \alpha)^{i j k l m} \Delta_{i} \Delta_{j} \Delta_{k} \Delta_{l} \Delta_{m} \\
= & \Delta_{s}(\mu \circ \alpha)^{s j k l m} \Delta_{j} \Delta_{k} \Delta_{l} \Delta_{m}+\Delta_{I}(\mu \circ \alpha)^{I j k l m} \Delta_{j} \Delta_{k} \Delta_{l} \Delta_{m}+\Delta_{\alpha}(\mu \circ \alpha)^{\alpha j k l m} \Delta_{j} \Delta_{k} \Delta_{l} \Delta_{m} \\
= & \Delta_{s}\left[(\mu \circ \alpha)^{s j k l m} \Delta_{j} \Delta_{k}+\Delta_{s}(\mu \circ \alpha)^{I l m} \Delta_{I}+(\mu \circ \alpha)^{\alpha k l m} \Delta_{\alpha} \Delta_{k}\right] \Delta_{l} \Delta_{m} \\
= & \Delta_{s}^{3}\left(-\Delta_{2}^{2}+\Delta_{3}^{2}+\ldots+\Delta_{q+3}^{2}\right)+\frac{\Delta_{s}}{16} \sum_{K=1}^{r} R_{K}^{2}(\hat{\Delta}) \\
& +\Delta_{s}^{2} \sum_{I=0}^{q+1} \Delta_{I}\left(2 \Delta_{s} \eta_{I I} \Delta_{I+2}+\frac{1}{2}{ }^{T} \hat{\Delta} \Gamma_{I} \hat{\Delta}\right) \\
& +\Delta_{s}^{2}\left(\sum_{I=0}^{q+1} \Delta_{I+2}{ }^{T} \hat{\Delta} \Gamma_{I} \hat{\Delta}\right)-\frac{\Delta_{s}}{4} \sum_{K=1}^{r} R_{K}(\hat{\Delta}){ }^{T} \hat{\Delta} \Omega_{K} \hat{\Delta} \\
= & \Delta_{s}^{3}\left(-\Delta_{2}^{2}+\Delta_{3}^{2}+\ldots+\Delta_{q+3}^{2}\right)+\frac{\Delta_{s}}{16} \sum_{K=1}^{r} R_{K}^{2}(\hat{\Delta}) \\
& +2 \Delta_{s}^{3} \sum_{I, J=0}^{q+1} \Delta_{I} \eta_{I J} \Delta_{J+2}+\frac{\Delta_{s}^{2}}{2} \sum_{I=0}^{q+1} \Delta_{I}{ }^{T} \hat{\Delta} \Gamma_{I} \hat{\Delta} \\
& +\Delta_{s}^{2} \sum_{I=0}^{q+1} \Delta_{I+2}^{T} \hat{\Delta} \Gamma_{I} \hat{\Delta}-\frac{\Delta_{s}}{4} \sum_{K=1}^{r} R_{K}^{2}(\hat{\Delta}) \\
= & \Delta_{s}^{3}\left[-\Delta_{2}\left(2 \Delta_{0}+\Delta_{2}\right)+\Delta_{3}\left(2 \Delta_{1}+\Delta_{3}\right)+\ldots+\Delta_{q+3}\left(2 \Delta_{q+1}+\Delta_{q+3}\right)\right] \\
& +\Delta_{s}^{2}\left(\frac{\Delta_{0}}{2}+\Delta_{2}\right)\left(\Delta_{q+4}^{2}+\ldots+\Delta_{q+3+m}^{2}\right) \\
& -\frac{3}{16} \Delta_{s} \sum_{K=1}^{r} R_{K}^{2}(\hat{\Delta})+\Delta_{s}^{2} \sum_{I=1}^{q+1}\left(\frac{\Delta_{I}}{2}+\Delta_{I+2}\right) Q_{I}(\hat{\Delta}) \\
= & \Delta_{s}^{3} \sum_{I=0}^{q+1} \eta_{I I} \Delta_{I+2}\left(2 \Delta_{I}+\Delta_{I+2}\right)+\Delta_{s}^{2}\left(\frac{\Delta_{0}}{2}+\Delta_{2}\right) \sum_{\alpha=1}^{m} \hat{\Delta}_{\alpha}^{2} \\
& 3 \sum_{K=1}^{r} R_{K}^{2}(\hat{\Delta})+\Delta_{s}^{2} \sum_{I=1}^{q+1}\left(\frac{\Delta_{I}}{2}+\Delta_{I+2}\right) Q_{I}(\hat{\Delta}), \\
& \\
& \\
&
\end{aligned}
$$

where in the last steps we have explicited all sums, and

$$
\begin{aligned}
\hat{\Delta} & :={ }^{T}\left(\hat{\Delta}_{1}, \ldots, \hat{\Delta}_{m}\right) \equiv^{T}\left(\Delta_{q+4}, \ldots, \Delta_{q+3+m}\right) ; \\
R_{K}(\hat{\Delta}) & :={ }^{T} \hat{\Delta} \Omega_{K} \hat{\Delta}=\sum_{\alpha, \beta=1}^{m}\left(\Omega_{K}\right)_{\alpha \beta} \hat{\Delta}_{\alpha} \hat{\Delta}_{\beta} ; \\
Q_{I}(\hat{\Delta}) & :={ }^{T} \hat{\Delta} \Gamma_{I} \hat{\Delta}=\sum_{\alpha, \beta=1}^{m}\left(\Gamma_{I}\right)_{\alpha \beta} \hat{\Delta}_{\alpha} \hat{\Delta}_{\beta} .
\end{aligned}
$$


We should remark that the following identifications of labels have been understood throughout:

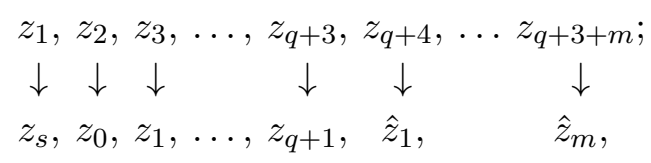

where $z_{0}, z_{1}, z_{2}, \ldots, z_{q+1}=z_{I}$, and $\hat{z}_{1}, \ldots, \hat{z}_{m}=\hat{z}_{\alpha} \equiv z_{\alpha}$.

Consequently, (6.46) yields that if

$$
\begin{gathered}
\left(\partial_{p} \Delta\right) \cdot(\mu \circ \alpha)\left(\partial_{p} \Delta\right)>0 \\
\mathbb{\Downarrow} \\
\Delta_{s} \sum_{I=0}^{q+1} \eta_{I I} \Delta_{I+2}\left(2 \Delta_{I}+\Delta_{I+2}\right)+\left(\frac{\Delta_{0}}{2}+\Delta_{2}\right) \sum_{\alpha=1}^{m} \hat{\Delta}_{\alpha}^{2}-\frac{3}{16 \Delta_{s}} \sum_{K=1}^{r} R_{K}^{2}(\hat{\Delta}) \\
+\sum_{I=1}^{q+1}\left(\frac{\Delta_{I}}{2}+\Delta_{I+2}\right) Q_{I}(\hat{\Delta})>0
\end{gathered}
$$

the solution to the system (2.6) reads, in vector notation,

$$
\xi=\frac{3}{2\left|\Delta_{s}\right|} \frac{(\mu \circ \alpha)\left(\partial_{p} \Delta\right)}{\sqrt{\left(\partial_{p} \Delta\right) \cdot(\mu \circ \alpha)\left(\partial_{p} \Delta\right)}},
$$

or, more explicitly (recall (4.41))

$$
\begin{aligned}
s & =\frac{3}{2\left|\Delta_{s}\right|} \frac{\left[\Delta_{s}^{2}\left(-\Delta_{2}^{2}+\Delta_{3}^{2}+\ldots+\Delta_{q+3}^{2}\right)+\frac{1}{16} \sum_{K=1}^{r} R_{K}^{2}(\hat{\Delta})\right]}{\sqrt{\left(\partial_{p} \Delta\right) \cdot(\mu \circ \alpha)\left(\partial_{p} \Delta\right)}} \\
x^{I} & =\frac{3}{2} \frac{\left|\Delta_{s}\right|\left(2 \Delta_{1} \eta_{I I} \Delta_{I+2}+\frac{1}{2}{ }^{T} \hat{\Delta} \Gamma_{I} \hat{\Delta}\right)}{\sqrt{\left(\partial_{p} \Delta\right) \cdot(\mu \circ \alpha)\left(\partial_{p} \Delta\right)}} ; \\
y^{\alpha} & =\frac{3}{2} \operatorname{sgn}\left(\Delta_{s}\right) \frac{\left[\Delta_{s} \sum_{I=0}^{q+1} \Delta_{I+2} \sum_{\beta=1}^{m}\left(\Gamma_{I}\right)_{\alpha \beta} z_{q+3+\beta}-\frac{1}{4} \sum_{K=1}^{r} R_{K}(\hat{z}) \sum_{\beta=1}^{m}\left(\Omega_{K}\right)_{\alpha \beta} z_{q+3+\beta}\right]}{\sqrt{\left(\partial_{p} \Delta\right) \cdot(\mu \circ \alpha)\left(\partial_{p} \Delta\right)}}
\end{aligned}
$$

where $\Delta_{i} \equiv \partial_{p^{i}} \Delta$ has been defined in (2.3) (see also (3.5) and (3.7)), and all sums have been made explicit in the numerators; the quantity $\left(\partial_{p} \Delta\right) \cdot(\mu \circ \alpha)\left(\partial_{p} \Delta\right)$ in the square root in the denominator is given by (6.46).

In order to prove that (6.53) is a solution to the BPS system (2.6), we compute

$$
\xi \cdot \partial_{p} \Delta=\frac{3}{2} \frac{\sqrt{\left(\partial_{p} \Delta\right) \cdot(\mu \circ \alpha)\left(\partial_{p} \Delta\right)}}{\left|\Delta_{S}\right|}=\mathcal{V}(\xi) .
$$

Thus, by recalling the Euler formula

$$
\mathcal{V}(\xi)=\frac{1}{3} \nabla \mathcal{V}(\xi) \cdot \xi
$$


one obtains that

$$
\begin{gathered}
0=\xi \cdot\left(\partial_{p} \Delta-\frac{1}{3} \nabla_{\mathcal{V}}(\xi)\right) \quad \forall \xi, \forall \partial_{p} \Delta ; \\
\Uparrow \\
\partial_{p} \Delta=\frac{1}{3} \nabla_{\mathcal{V}}(\xi)=\frac{1}{3 !} d_{i j k} x^{j} x^{k},
\end{gathered}
$$

which is the BPS system (2.6) itself.

Thus, in all models explicitly treated below, after the checking that the invertibility condition enounced in section 6.2 holds true, the crucial data to be known are the symmetric $m \times m \Gamma$-matrices and the symmetric, auxiliary $m \times m$ matrices $\Omega_{K}$ such that (6.6) and (6.8) both hold true.

\subsubsection{The symmetric case}

It should be remarked that those homogeneous $d$-spaces $[53,56]$ in which the invertibility condition enounced in section 6.2 is satisfied include the noteworthy class of homogeneous symmetric $d$-spaces (cfr. section 4.1), in which it holds that

$$
(\mu \circ \alpha)^{i}\left(\partial_{p} \Delta\right)=(\mu \circ \alpha)^{i j k l m} \Delta_{j} \Delta_{k} \Delta_{l} \Delta_{m}=2 \Delta_{S}^{2} d^{i j k} \Delta_{j} \Delta_{k}
$$

where $d^{i j k}$ is defined in (4.15)-(4.19), and in the case of symmetric $d$-spaces it is a constant (numerical) tensor (i.e., it does not depend on any scalar fields' degree of freedom). (6.61) implies that

$$
\begin{gathered}
\left(\partial_{p} \Delta\right) \cdot(\mu \circ \alpha)\left(\partial_{p} \Delta\right)=(\mu \circ \alpha)^{i j k l m} \Delta_{i} \Delta_{j} \Delta_{k} \Delta_{l} \Delta_{m}=2 \Delta_{S}^{2} d^{i j k} \Delta_{i} \Delta_{j} \Delta_{k} ; \\
\Downarrow \\
\left(\partial_{p} \Delta\right) \cdot(\mu \circ \alpha)\left(\partial_{p} \Delta\right)>0 \quad \Leftrightarrow \quad d^{i j k} \Delta_{i} \Delta_{j} \Delta_{k}>0 .
\end{gathered}
$$

Indeed, by plugging (6.62) into (6.53), one obtains

$$
\xi^{i}=\frac{3}{2\left|\Delta_{s}\right|} \frac{(\mu \circ \alpha)^{i}\left(\partial_{p} \Delta\right)}{\sqrt{\left(\partial_{p} \Delta\right) \cdot(\mu \circ \alpha)\left(\partial_{p} \Delta\right)}}=\frac{3}{\sqrt{2}} \frac{d^{i j k} \Delta_{j} \Delta_{k}}{\sqrt{d^{m n p} \Delta_{m} \Delta_{n} \Delta_{p}}}
$$

thus matching (4.8) (recalling (4.41)).

\subsection{BPS black hole entropy and attractors}

Let us recall that $\xi$ enters the expression (2.2) of the black hole entropy only through the quantity (2.8), and (6.57) holds true. Remarkably, by virtue of (2.8), the solutions of the BPS system (2.6) enter the expression of the black hole entropy only through the square of the quantity (6.57):

$$
\left(\xi \cdot \partial_{p} \Delta\right)^{2}=\mathcal{V}^{2}(\xi)=\frac{9}{4} \frac{\left(\partial_{p} \Delta\right) \cdot(\mu \circ \alpha)\left(\partial_{p} \Delta\right)}{\Delta_{s}^{2}}
$$


Then, by recalling (2.2), the Bekenstein-Hawking entropy of static, spherically symmetric, BPS extremal dyonic black holes in the model under consideration of $\mathcal{N}=2, D=4$ Maxwell-Einstein supergravity has the following expression:

$$
\frac{S}{\pi}=\frac{1}{3\left|p^{0}\right|} \sqrt{3 \frac{\left(\partial_{p} \Delta\right) \cdot(\mu \circ \alpha)\left(\partial_{p} \Delta\right)}{\Delta_{s}^{2}}-9\left[p^{0}(p \cdot q)-2 I_{3}(p)\right]^{2}} .
$$

In both formulæ (6.65) and (6.66) the scalar product $\left(\partial_{p} \Delta\right) \cdot(\mu \circ \alpha)\left(\partial_{p} \Delta\right)$ is given by (6.46). Note that, as it must be, $S$ (6.66) is a homogeneous positive function of degree 2 in the black hole charges. The consistency conditions for (6.65) and the BPS black hole entropy (6.66) to hold formally read as follows:

$$
\left\{\begin{aligned}
\left(\partial_{p} \Delta\right) \cdot(\mu \circ \alpha)\left(\partial_{p} \Delta\right)>0 ; \\
\left(\partial_{p} \Delta\right) \cdot(\mu \circ \alpha)\left(\partial_{p} \Delta\right)-3\left[p^{0}(p \cdot q)-2 I_{3}(p)\right]^{2} \Delta_{s}^{2}>0 ; \\
\Downarrow \\
\left(\partial_{p} \Delta\right) \cdot(\mu \circ \alpha)\left(\partial_{p} \Delta\right)>3\left[p^{0}(p \cdot q)-2 I_{3}(p)\right]^{2} \Delta_{s}^{2},
\end{aligned}\right.
$$

where again the scalar product $\left(\partial_{p} \Delta\right) \cdot(\mu \circ \alpha)\left(\partial_{p} \Delta\right)$ is given by (6.46).

By exploiting the results (6.37) and (6.65) and defining

$$
\begin{aligned}
\mathcal{Q} & ={ }^{T}\left(p^{0}, p^{i}, q_{0}, q_{i}\right) ; \\
z_{H}(\mathcal{Q}) & \equiv\left\{z_{H}^{i}(\mathcal{Q})\right\}_{i}:={ }^{T}\left(z_{H}^{s}(\mathcal{Q}), z_{H}^{I}(\mathcal{Q}), z_{H}^{\alpha}(\mathcal{Q})\right) ; \\
p & \equiv\left\{p^{i}\right\}_{i}:={ }^{T}\left(p^{s}, p^{I}, p^{\alpha}\right),
\end{aligned}
$$

the expression of BPS attractor points (2.13) is given, in vector notation, by

$$
z_{H}(\mathcal{Q})=\frac{3}{2} \frac{(\mu \circ \alpha)\left(\partial_{p} \Delta\right)}{\left(\partial_{p} \Delta\right) \cdot(\mu \circ \alpha)\left(\partial_{p} \Delta\right)}\left[\frac{p^{0}(p \cdot q)-2 I_{3}(p)}{p^{0}}-\mathbf{i} \frac{3}{2} \frac{S}{\pi}\right]+\frac{p}{p^{0}},
$$

or, more explicitly:

$$
\begin{aligned}
z_{H}^{s}(\mathcal{Q})= & \frac{3}{2} \frac{\left[\Delta_{s}^{2}\left(-\Delta_{2}^{2}+\Delta_{3}^{2}+\ldots+\Delta_{q+3}^{2}\right)+\frac{1}{16} \sum_{K=1}^{r} R_{K}^{2}(\hat{\Delta})\right]}{\left(\partial_{p} \Delta\right) \cdot(\mu \circ \alpha)\left(\partial_{p} \Delta\right)}\left[\frac{p^{0}(p \cdot q)-2 I_{3}(p)}{p^{0}}-\mathbf{i} \frac{3}{2} \frac{S}{\pi}\right]+\frac{p^{s}}{p^{0}} \\
z_{H}^{I}(\mathcal{Q})= & \frac{3}{2} \frac{\Delta_{s}^{2}\left(2 \Delta_{s} \eta_{I I} \Delta_{I+2}+\frac{1}{2} T \hat{z} \Gamma_{I} \hat{z}\right)}{\left(\partial_{p} \Delta\right) \cdot(\mu \circ \alpha)\left(\partial_{p} \Delta\right)}\left[\frac{p^{0}(p \cdot q)-2 I_{3}(p)}{p^{0}}-\mathbf{i} \frac{3}{2} \frac{S}{\pi}\right]+\frac{p^{I}}{p^{0}} ; \\
z_{H}^{\alpha}(\mathcal{Q})= & \frac{3}{2} \frac{\left[\Delta_{s} \sum_{I=0}^{q+1} \Delta_{I+2} \sum_{\beta=1}^{m}\left(\Gamma_{I}\right)_{\alpha \beta} \Delta_{q+3+\beta}-\frac{1}{4} \sum_{K=1}^{r} R_{K}(\hat{\Delta}) \sum_{\beta=1}^{m}\left(\Omega_{K}\right)_{\alpha \beta} \Delta_{q+3+\beta}\right]}{\left(\partial_{p} \Delta\right) \cdot(\mu \circ \alpha)\left(\partial_{p} \Delta\right)} \\
& \cdot\left[\frac{p^{0}(p \cdot q)-2 I_{3}(p)}{p^{0}}-\mathbf{i} \frac{3}{2} \frac{S}{\pi}\right]+\frac{p^{\alpha}}{p^{0}},
\end{aligned}
$$

where all sums are explicitly indicated in the numerator of $(6.75)$, and $\left(\partial_{p} \Delta\right) \cdot(\mu \circ \alpha)\left(\partial_{p} \Delta\right)$ and $S$ are respectively given by (6.46) and (6.66). 
Again, by considering the general formula (6.66) in homogeneous symmetric models in which the results discussed in section 6.5.1 as well as the adjoint identity (4.1) hold true, it can be checked that the entropy acquires the simple form given by (4.12) (see discussion in section 4.1).

\subsection{Complete models: $r=0$}

The classification, completed in [53] (see also [56]), shows that any $L(q, 1,0) \equiv L(q, 1)$, $q \geqslant-1$ model of homogeneous very special geometry is defined by $\Gamma_{0}=\mathbb{I}_{m}$ and a Clifford set $\Gamma$-matrices, of size $m \times m$, namely by $\left\{\Gamma_{1}, \ldots, \Gamma_{q+1}\right\}$, where $m=\mathcal{D}_{q+1}$ (given e.g. in table 1 of [56] and in (4.26)). For fixed $q$, such matrices are unique (up to a choice of basis in $\left.\mathbb{R}^{m}\right)$. We will choose in section 8.1a Clifford set $\left\{\Gamma_{I}\right\}_{I=1, \ldots, 9}$ of symmetric $\Gamma$-matrices. The associated quadratic forms $\left\{Q_{I}\right\}_{I \neq 0}$ 's have the additional pleasant property that putting the last $m / 2$ coordinate $y^{\alpha}$ 's equal to zero, some quadrics vanish identically, whereas the non-vanishing ones are the quadrics associated to an $L\left(q^{\prime}, 1\right)$ model with $q^{\prime}<8$.

A complete model is defined to be a model in which $r=0$ in the invertibility condition enounced in section 6.2. Then, (6.8) implies that the associated quadratic forms satisfy a Lorentzian quadratic relation

$$
-Q_{0}^{2}(y)+Q_{1}^{2}(y)+\ldots+Q_{q+1}^{2}(y)=0 \Leftrightarrow q(Q(y))=0,
$$

where (4.58) has been recalled. Defining the associated cubic form $\mathcal{V}$ as in (6.5), the main result then states that the gradient map $\nabla \mathcal{V}$ will be invertible, with the (birational) inverse map being polynomial of degree 2 .

Indeed, since for $r=0$ all coordinate functions of the map $\alpha$ are multiples of $z_{1}$ and $\mu$ is homogeneous, so, as mentioned above, one can redefine $\alpha$ to be the identity map. As a consequence, $\mu$, which is given by quadratic polynomials, is the birational inverse of $\nabla_{\mathcal{V}}$; cfr. (6.20), which can be regarded as a consequence of the so-called "adjoint identity" (4.1) of cubic Jordan algebras.

The only ${ }^{23}$ complete models known to us have $q=1,2,4,8$ and $m=2 q$, so $N=$ $3 q+3=6,9,15,27$ respectively; such models have been discussed at the end of section 4.3.1, and they will be further discussed below. In these models, which correspond to the "magic" class of symmetric $d$-manifolds [76-78], the cubic forms are the well known norm forms on simple cubic Jordan algebras $J_{3}^{\mathbb{A}}$, for $\mathbb{A}=\mathbb{R}, \mathbb{C}, \mathbb{H}, \mathbb{O}$ [117]. These complete models correspond to the models $L(q, 1)[53,56]$ with $q=1,2,4,8=\operatorname{dim}_{\mathbb{R}} \mathbb{A}$ for $\mathbb{A}=\mathbb{R}, \mathbb{C}, \mathbb{H}, \mathbb{O}$ respectively, provided that the $(q+2)$-dimensional vector has a (mostly plus) Lorentzian signature $\left(1_{-},(q+1)_{+}\right)$, which can always be arranged.

As we will see in section 13, also quadratic forms in $q+2=4,6,10$ dimensions of Kleinian signatures $\left(2_{+}, 2_{-}\right),\left(3_{+}, 3_{-}\right)$and $\left(5_{+}, 5_{-}\right)$can be considered: they are associated to simple cubic Jordan algebras over split composition algebras $J_{3}^{\mathbb{A}_{s}}$, for $\mathbb{A}=\mathbb{C}_{s}, \mathbb{H}_{s}, \mathbb{O}_{s}$. Moreover, they correspond to non-supersymmetric Maxwell-Einstein theories (for $\mathbb{C}_{s}$ and $\mathbb{H}_{s}$ ), as well as to maximal supergravity (in the case of $\mathbb{O}_{s}$ ); cfr. [101, 102].

\footnotetext{
${ }^{23}$ Actually, (4.1) holds also for $J_{3}=\mathbb{R}$ (corresponding to the $T^{3}$ model of $\mathcal{N}=2, D=4$ supergravity) as well as for semi-simple cubic Jordan algebras (named "spin factors") $\mathbb{R} \oplus \boldsymbol{\Gamma}_{a, b}$ [86]. Therefore, strictly speaking, also these models should be complete.
} 


\section{$7 \quad$ Verifying the inverse map}

In this section we prove that, if the condition enounced in section 6.2 is satisfied, then the formulas in section 6.4 indeed provide the (birational) inverse map of the gradient map of the cubic form $\mathcal{V}$ under consideration.

\subsection{Factorization of $R_{K}$ 's}

First of all, we derive a useful property of the quadratic polynomials $R_{K}$ 's, defined by the extra symmetric matrices $\Omega_{K}$, with $1 \leqslant K \leqslant r$ (with $r \geqslant 0$ ), of size $m$.

The last $m=2^{g}$ coordinate functions of $\nabla \mathcal{V}$ are denoted by $\left(y={ }^{T}\left(y^{1}, \ldots, y^{m}\right)\right)$

$$
\nabla_{y} \mathcal{V}:={ }^{T}\left(\frac{\partial \mathcal{V}}{\partial y^{1}}, \ldots, \frac{\partial \mathcal{V}}{\partial y^{m}}\right)=2^{T} y\left(\sum_{I=0}^{q+1} x^{I} \Gamma_{I}\right)
$$

We show that, upon substituting $\nabla_{y} \mathcal{V}$ into $R_{K}(6.7)$, the following factorization holds:

$$
R_{K}\left(\nabla_{y} \mathcal{V}\right)=-4 q(x) R_{K}(y)
$$

In fact, by definition, one has:

$$
R_{K}\left(\nabla_{y} \mathcal{V}\right)=4^{T} y\left(\sum_{I=0}^{q+1} x^{I} \Gamma_{I}\right) \cdot \Omega_{K} \cdot\left(\sum_{J=0}^{q+1} x^{J} \Gamma_{J}\right) y=4^{T} y\left(\sum_{I, J=0}^{q+1} x^{I} x^{J}\left(\Gamma_{I} \Omega_{K} \Gamma_{J}\right)\right) y
$$

Recall that $\Gamma_{0}=\mathbb{I}_{m}$ and that from (6.6) we have $\Gamma_{I} \Omega_{K}=-\Omega_{K} \Gamma_{I}$ for $I=1, \ldots, q+1$. Therefore, for $I>0$ it holds that

$$
\Gamma_{0} \Omega_{K} \Gamma_{0}=\Omega_{K}, \quad \Gamma_{0} \Omega_{K} \Gamma_{I}=-\Gamma_{I} \Omega_{K}=-\Gamma_{I} \Omega_{K} \Gamma_{0} .
$$

If $I, J>0$ and $I \neq J$, we have $\Gamma_{I} \Gamma_{J}=-\Gamma_{J} \Gamma_{I}$ since we have a Clifford set, and thus

$$
\Gamma_{I} \Omega_{K} \Gamma_{J}=-\Gamma_{I} \Gamma_{J} \Omega_{K}=\Gamma_{J} \Gamma_{I} \Omega_{K}=-\Gamma_{J} \Omega_{K} \Gamma_{I}
$$

Furthermore, when $I=J>0$ we have $\Gamma_{I} \Gamma_{J}=\Gamma_{I}^{2}=\mathbb{I}_{m}$, and thus

$$
\Gamma_{I} \Omega_{K} \Gamma_{J}=-\Gamma_{I} \Gamma_{J} \Omega_{K}=-\Omega_{K} .
$$

Therefore, all terms with $I \neq J$ in (7.3) cancel, and we are left with

$$
\begin{aligned}
R_{K}\left(\nabla_{y} \mathcal{V}\right) & =4^{T} y\left(\left(x^{0}\right)^{2} \Omega_{K}-\sum_{I=1}^{q+1}\left(x^{I}\right)^{2} \Omega_{K}\right) y \\
& =4\left(\left(x^{0}\right)^{2}-\left(\left(x^{1}\right)^{2}+\ldots\left(x^{q+1}\right)^{2}\right)\right)^{T} y \Omega_{K} y=-4 q(x) R_{K}(y)
\end{aligned}
$$

which proves (7.2). 


\subsection{The map $\alpha \circ \nabla_{\mathcal{V}}$}

For $\xi=(s, x, y) \in \mathbb{R}^{q+3+m}$ (cfr. (4.41)), we now verify that the image $(t, u, v, w)=\alpha(\nabla \mathcal{V}(\xi))$ in $\mathbb{R}^{q+3+m+r}$ is given by (6.16).

The first $q+3+m$ components of $\alpha(z)$ are the $z_{1} z_{a}$ 's, with $a=1, \ldots, q+3+m$. Since the first component of $\nabla_{\mathcal{V}}$ is $\partial \mathcal{V} / \partial s=q(x)$, we see that the first $q+3+m$ components of $\alpha(\nabla \mathcal{V}(\xi)):=\alpha \circ \nabla \mathcal{V}(\xi)$ are $q(x) \nabla \mathcal{V}(\xi)$.

The last $r$ components of $\alpha(z)$ are the $R_{K}$ 's, evaluated on the last $m$ variables. Hence, the last $r$ components of $\alpha \circ \nabla_{\mathcal{V}}(\xi)$ are the $R_{K}$ 's evaluated on $m$-vector $\nabla_{y} \mathcal{V}$. From (7.2) we see that these components are $-4 q(x) R_{K}(y), K=1, \ldots, r$.

Thus, all components of $\alpha \circ \nabla_{\mathcal{V}}(\xi)$ are stated as in (6.16). For later convenience, we write these components explicitly, with $I=1, \ldots, q+1, \alpha=1, \ldots, m$ and $K=1, \ldots, r$ :

$$
\begin{aligned}
t & =q(x)^{2} ; \\
u_{0} & =q(x)\left(-2 x^{0} s+Q_{0}(y)\right) ; \\
u_{I} & =q(x)\left(2 x^{I} s+Q_{I}(y)\right) ; \\
v_{\alpha} & =2 q(x)\left(\sum_{I=0}^{q+1} x^{I} \Gamma_{I}\right)_{\alpha \beta} y^{\beta} ; \\
w_{K} & =-4 q(x) R_{K}(y) .
\end{aligned}
$$

\subsection{The map $\mu \circ \alpha \circ \nabla_{\mathcal{V}}$}

We now aim at proving (6.18), which in particular implies that the composition $\mu \circ \alpha$ is the birational inverse of the gradient map $\nabla_{\mathcal{V}}$.

To do so, we first compute $\mu(\alpha(\nabla \mathcal{V}(\xi)))$ with $\xi={ }^{T}(s, x, y)$ (cfr. (4.41)). We already computed $\left(\alpha\left(\nabla_{\mathcal{V}}(\xi)\right)\right.$ in (6.16) and (7.8)-(7.12), so it remains to evaluate the map $\mu$, defined in $(6.17)$, on ${ }^{T}(t, u, v, w)=\alpha\left(\nabla_{\mathcal{V}}(\xi)\right)$.

From (6.17), the first component of $\mu$ is $q(u)+\frac{1}{16} \sum_{K=1}^{r} w_{K}^{2}$, and it holds that

$$
\frac{1}{16} \sum_{K=1}^{r} w_{K}^{2}=\frac{1}{16} \sum_{K=1}^{r}\left(-4 q(x) R_{K}(y)\right)^{2}=q(x)^{2} \sum_{K=1}^{r} R_{K}(y)^{2}
$$

Next, from (6.17) the second component of $\mu$ is $-2 t u_{0}+\frac{1}{2} Q_{0}(v)$, and it holds that

$$
2 t u_{0}=-2 q(x)^{3}\left(-2 s x^{0}+Q_{0}(y)\right)=2 q(x)^{3}\left(2 s x^{0}-Q_{0}(y)\right) .
$$

Therefore, the next $J$ components of $\mu(J=1, \ldots, q+1)$ are, from $(6.17), 2 t u_{J}+\frac{1}{2} Q_{J}(v)$, and it holds that

$$
2 t u_{J}=2 q(x)^{3}\left(2 s x^{J}+Q_{J}(y)\right) .
$$


Therefore, from (6.17), (7.8)-(7.12) and (7.13)-(7.15), one obtains

$$
\left(\mu \circ \alpha \circ \nabla_{\mathcal{V}}\right)(s, x, y)=q(x)^{2}\left(\begin{array}{l}
q\left(-2 s x^{0}+Q_{0}(y), 2 s x^{1}+Q_{1}(y), \ldots, 2 s x^{q+1}+Q_{q+1}(y)\right) \\
\quad+\sum_{K=1}^{r} R_{K}(y)^{2} \\
2 q(x)\left(2 s x^{0}-Q_{0}(y)\right)+\frac{1}{2} Q_{0}\left(\nabla_{y} \mathcal{V}\right) \\
2 q(x)\left(2 s x^{1}+Q_{1}(y)\right)+\frac{1}{2} Q_{1}\left(\nabla_{y} \mathcal{V}\right) \\
\vdots \\
2 q(x)\left(2 s x^{q+1}+Q_{q+1}(y)\right)+\frac{1}{2} Q_{q+1}\left(\nabla_{y} \mathcal{V}\right) \\
\left(\sum_{I=0}^{q+1} \mathcal{V}_{I} \Gamma_{I}\right) \nabla_{y} \mathcal{V}+\left(\sum_{K=1}^{r} R_{K}(y) \Omega_{K}\right) \nabla_{y} \mathcal{V}
\end{array}\right) .
$$

To verify (6.18), the treatment can be split in three parts, respectively concerning the first, $s$-component, the middle $q+2$ components and the last $m$ components of $\mu\left(\alpha\left(\nabla_{\mathcal{V}}(s, x, y)\right)\right) \equiv\left(\mu \circ \alpha \circ \nabla_{\mathcal{V}}\right)(s, x, y) \equiv \mu \circ \alpha \circ \nabla_{\mathcal{V}}(\xi):$

1. For what concerns the first, $s$ - component of the map $(\mu \circ \alpha \circ \nabla \mathcal{V})(s, x, y)$, one needs to show that

$$
4 \mathcal{V} s=q\left(-2 s x^{0}+Q_{0}(y), 2 s x^{1}+Q_{1}(y), \ldots, 2 s x^{q+1}+Q_{q+1}(y)\right)+\sum_{K=1}^{r} R_{K}(y)^{2} .
$$

We start and consider

$$
\begin{aligned}
& q\left(-2 s x^{0}+Q_{0}(y), 2 s x^{1}+Q_{1}(y), \ldots, 2 s x^{q+1}+Q_{q+1}(y)\right) \\
= & -\left(-2 s x^{0}+Q_{0}(y)\right)^{2}+\sum_{I=1}^{q+1}\left(2 s x^{I}+Q_{I}(y)\right)^{2} \\
= & 4 s^{2} q(x)+4 s \sum_{I=0}^{q+1} x^{I} Q_{I}(y)-Q_{0}(y)^{2}+\sum_{I=1}^{q+1} Q_{I}(y)^{2} \\
= & 4 \mathcal{V} s-Q_{0}(y)^{2}+\sum_{I=1}^{q+1} Q_{I}(y)^{2} .
\end{aligned}
$$

Using the identity (6.8), one finds

$$
\sum_{K=1}^{r} R_{K}(y)^{2}=Q_{0}(y)^{2}-\sum_{I=1}^{q+1} Q_{I}(y)^{2}
$$

and thus (7.18) proves (7.17).

2. For the next $q+2$ components of the map $(\mu \circ \alpha \circ \nabla \mathcal{V})(s, x, y)$, one needs to prove that (cfr. (7.16)):

$$
\left\{\begin{array}{l}
4 \mathcal{V} x^{0}=2 q(x)\left(2 s x^{0}-Q_{0}(y)\right)+\frac{1}{2} Q_{0}\left(\nabla_{y} \mathcal{V}\right), \\
4 \mathcal{V} x^{I}=2 q(x)\left(2 s x^{I}+Q_{I}(y)\right)+\frac{1}{2} Q_{I}\left(\nabla_{y} \mathcal{V}\right), \quad I=1, \ldots, q+1 .
\end{array}\right.
$$


We claim that

$$
\frac{1}{2} Q_{I}\left(\nabla_{y} \mathcal{V}\right)=\left\{\begin{array}{rll}
2 q(x) Q_{0}(y)+4 x^{0} \sum_{J=0}^{q+1} x^{J} Q_{J}(y) & \text { if } \quad I=0 \\
-2 q(x) Q_{I}(y)+4 x^{I} \sum_{J=0}^{q+1} x^{J} Q_{J}(y) & \text { if } \quad I=1, \ldots, q+1 .
\end{array}\right.
$$

Indeed, it holds that

$$
\frac{1}{2} Q_{I}\left(\nabla_{y} \mathcal{V}\right)=\frac{1}{2} Q_{I}\left(2^{T} y\left(\sum_{J=0}^{q+1} x^{J} \Gamma_{J}\right)\right)=2^{T} y\left(\sum_{J=0}^{q+1} x^{J} \Gamma_{J}\right) \Gamma_{I}\left(\sum_{J^{\prime}=0}^{q+1} x^{J^{\prime}} \Gamma_{J^{\prime}}\right) y
$$

If $I=0$, then $\Gamma_{0}=\mathbb{I}_{m}$ and thus (7.22), combined with (5.12), leads to

$$
\begin{aligned}
\frac{1}{2} Q_{0}\left(\nabla_{y} \mathcal{V}\right) & =2^{T} y\left(\sum_{J=0}^{q+1} x^{J} \Gamma_{J}\right) \Gamma_{0}\left(\sum_{J^{\prime}=0}^{q+1} x^{J^{\prime}} \Gamma_{J^{\prime}}\right) y \\
& =2^{T} y\left(x^{0} \mathbb{I}_{m}+\sum_{J=1}^{q+1} x^{J} \Gamma_{J}\right)\left(2 x^{0} \mathbb{I}_{m}-x^{0} \mathbb{I}_{m}+\sum_{J^{\prime}=1}^{q+1} x^{J^{\prime}} \Gamma_{J^{\prime}}\right) y \\
& =4 x^{0 T} y\left(\sum_{J=0}^{q+1} x^{J} \Gamma_{J}\right) y+2\left(-\left(x^{0}\right)^{2}+\left(x^{1}\right)^{2}+\ldots+\left(x^{q+1}\right)^{2}\right)^{T} y \mathbb{I}_{m} y \\
& =4 x^{0} \sum_{J=0}^{q+1} x^{J} Q_{J}(y)+2 q(x) Q_{0}(y) .
\end{aligned}
$$

On the other hand, if $I=1, \ldots, q+1$ then $\Gamma_{I}$ and $\Gamma_{J}$ commute only for $J=0$ or $J=I$, and they anti-commute otherwise; consequently, by moving $\Gamma_{I}$ to the right, it follows that

$$
\begin{aligned}
\frac{1}{2} Q_{I}\left(\nabla_{y} \mathcal{V}\right) & =2^{T} y\left(\sum_{J=0}^{q+1} x^{J} \Gamma_{J}\right) \Gamma_{I}\left(\sum_{J^{\prime}=0}^{q+1} x^{J^{\prime}} \Gamma_{J^{\prime}}\right) y \\
& =2^{T} y\left(x^{0} \mathbb{I}_{m}+\sum_{J=1}^{q+1} x^{J} \Gamma_{J}\right)\left(x^{0} \mathbb{I}_{m}-\left(\sum_{J^{\prime}=1}^{q+1} x^{J^{\prime}} \Gamma_{J^{\prime}}\right)+2 x^{I} \Gamma_{I}\right) \Gamma_{I} y \\
& =2\left[\left(x^{0}\right)^{2}-\left(\left(x^{1}\right)^{2}+\ldots+\left(x^{q+1}\right)^{2}\right)\right]{ }^{T} y \Gamma_{I} y+4 x^{I} T^{T} y\left(\sum_{J=0}^{q+1} x^{J} \Gamma_{J}\right) \Gamma_{I}^{2} y \\
& =-2 q(x) Q_{I}(y)+4 x^{I} \sum_{J=0}^{q+1} x^{J} Q_{J}(y) .
\end{aligned}
$$

Hence, the claim (7.21) is proven. Now, we show that the equalities in (7.20) follow from the claim (7.21). In fact, by recalling (6.5) for $I=0$ one obtains

$$
\begin{aligned}
& 2 q(x)\left(2 s x^{0}-Q_{0}(y)\right)+\frac{1}{2} Q_{0}\left(\nabla_{y} \mathcal{V}\right) \\
= & 4 s q(x) x^{0}-2 q(x) Q_{0}(y)+2 q(x) Q_{0}(y)+4 x^{0} \sum_{J=0}^{q+1} x^{J} Q_{J}(y)=4 x^{0} \mathcal{V} .
\end{aligned}
$$


Analogously, for $I=1, \ldots, q+1$ it holds that

$$
\begin{aligned}
& 2 q(x)\left(2 x^{I}+Q_{I}(y)\right)+\frac{1}{2} Q_{I}\left(\nabla_{y} \mathcal{V}\right) \\
= & 4 s q(x) x^{I}+2 q(x) Q_{I}(y)-2 q(x) Q_{I}(y)+4 x^{I} \sum_{J=0}^{q+1} x^{J} Q_{J}(y)=4 x^{I} \mathcal{V} .
\end{aligned}
$$

This concludes the verification for the $q+2$ components under consideration.

3. Finally, for the last $m$ components of $(\mu \circ \alpha \circ \nabla \mathcal{V})(s, x, y)$ one must check that

$$
4 \mathcal{V} y=\left(\sum_{I=0}^{q+1} \mathcal{V}_{I} \Gamma_{I}+\sum_{K=1}^{r} R_{K}(y) \Omega_{K}\right) \nabla_{y} \mathcal{V}
$$

First of all, we substitute $\mathcal{V}_{0}=-2 s x^{0}+Q_{0}(y)$ and $\mathcal{V}_{I}=2 s x^{I}+Q_{I}(y)$ for $I=1, \ldots, q+1$, in the r.h.s. of (7.27), obtaining

$$
\sum_{I=0}^{q+1} \mathcal{V}_{I} \Gamma_{I}=2 s\left(-x^{0} \Gamma_{0}+x^{1} \Gamma_{1}+\ldots+x^{q+1} \Gamma_{q+1}\right)+\sum_{I=0}^{q+1} Q_{I}(y) \Gamma_{I} .
$$

Thus, since $\mathcal{V}=s q(x)+\sum_{I=0}^{q+1} x^{I} Q_{I}(y)$, the formula (7.27) follows if we verify the following two identities:

$$
\begin{aligned}
4 s q(x) y & =2 s\left(-x^{0} \Gamma_{0}+x^{1} \Gamma_{1}+\ldots+x^{q+1} \Gamma_{q+1}\right) \nabla_{y} \mathcal{V} ; \\
4\left(\sum_{I=0}^{q+1} x^{I} Q_{I}(y)\right) y & =\left(\sum_{I=0}^{q+1} Q_{I}(y) \Gamma_{I}+\sum_{K=1}^{r} R_{K}(y) \Omega_{K}\right) \nabla_{y} \mathcal{V} .
\end{aligned}
$$

We recall that $\nabla_{y} \mathcal{V}=2\left(\sum_{I=0}^{q+1} x^{I} \Gamma_{I}\right) y$. The first identity (7.29) is easy to verify by substituting this and using (5.12); in fact, its r.h.s. can be elaborated as follows:

$$
\begin{aligned}
& 4 s\left(-x^{0} \Gamma_{0}+x^{1} \Gamma_{1}+\ldots+x^{q+1} \Gamma_{q+1}\right)\left(x^{0} \Gamma_{0}+x^{1} \Gamma_{1}+\ldots+x^{q+1} \Gamma_{q+1}\right) y \\
= & 4 s\left(-\left(x^{0}\right)^{2}+\left(x^{1}\right)^{2}+\ldots+\left(x^{q+1}\right)^{2}\right) y=4 s q(x) y .
\end{aligned}
$$

In order to prove the second identity (7.30), we observe that, since $\Gamma_{I}^{2}=\mathbb{I}_{m}$, the first term in its r.h.s. can be elaborated as

$$
\left(\sum_{J=0}^{q+1} Q_{J}(y) \Gamma_{J}\right)\left(2 \sum_{I=0}^{q+1} x^{I} \Gamma_{I}\right) y=2\left(\sum_{I=0}^{q+1} x^{I} Q_{I}(y)\right) y+2 \sum_{I=0}^{q+1} x^{I} \sum_{I \neq J=0}^{q+1} Q_{J}(y) \Gamma_{J} \Gamma_{I} y .
$$

The second term in the r.h.s. of (7.30) reads

$$
\left(\sum_{K=1}^{r} R_{K}(y) \Omega_{K}\right)\left(2 \sum_{I=0}^{q+1} x^{I} \Gamma_{I}\right) y=2 \sum_{I=0}^{q+1} x^{I}\left(\sum_{K=1}^{r} R_{K}(y) \Omega_{K}\right) \Gamma_{I} .
$$

So, the second identity (7.30) follows from (7.32) and (7.33) if we can show that

$$
\left(\sum_{I=0}^{q+1} x^{I} Q_{I}(y)\right) y=\sum_{I=0}^{q+1} x^{I}\left(\left(\sum_{I \neq J=0}^{q+1} Q_{J}(y) \Gamma_{J}\right)+\left(\sum_{K=1}^{r} R_{K}(y) \Omega_{K}\right)\right) \Gamma_{I} y,
$$


or, comparing the (matrix) coefficients of the $x^{I}$ 's, equivalently, for all $I=0,1, \ldots, q+1$ :

$$
Q_{I}(y)=\left(\left(\sum_{I \neq J=0}^{q+1} Q_{J}(y) \Gamma_{J}\right)+\left(\sum_{K=1}^{r} R_{K}(y) \Omega_{K}\right)\right) \Gamma_{I} .
$$

To verify (7.35), we use the identity (6.8), which we write as

$$
\mathbf{F}(y):=-Q_{0}(y)^{2}+Q_{1}(y)^{2}+\ldots+Q_{q+1}(y)^{2}+R_{1}(y)^{2}+\ldots+R_{r}(y)^{2}=0,
$$

with, as above, $Q_{I}(y)={ }^{T} y \Gamma_{I} y, R_{K}(y):={ }^{T} y \Omega_{K} y$; note that $\mathbf{F}(y)$ is identically zero as a polynomial in $y=\left(y^{1}, \ldots, y^{m}\right)$. Therefore all partial derivatives of $\mathbf{F}$ w.r.t. the $y^{\alpha}$ are also identically zero (as cubics in $y$ ). Notice that

$$
\begin{aligned}
0 & =\nabla_{y} \mathbf{F}(y) \\
& =2\left(-Q_{0}(y) \Gamma_{0}+Q_{1}(y) \Gamma_{1}+\ldots+Q_{q+1}(y) \Gamma_{q+1}+R_{1}(y) \Omega_{1}+\ldots+R_{r}(y) \Omega_{r}\right) y .
\end{aligned}
$$

Multiplying (7.37) from the left by one of the $\Gamma_{I}$ 's $(I=1, \ldots, q+1)$ and using $\Gamma_{I} \Gamma_{J}=-\Gamma_{J} \Gamma_{I}$ if $J \neq 0, I$ and $\Gamma_{I} \Omega_{K}=-\Omega_{K} \Gamma_{I}$, we get:

$$
\begin{aligned}
0= & \Gamma_{I} \nabla_{y} \mathbf{F}(y) \\
= & 2\left(-Q_{0}(y) \Gamma_{0}-Q_{1}(y) \Gamma_{1}-\ldots+Q_{I}(y) \Gamma_{I}-\ldots-Q_{q+1}(y) \Gamma_{q+1}\right. \\
& \left.-R_{1}(y) \Omega_{1}-\ldots-R_{r}(y) \Omega_{r}\right) \Gamma_{I} y .
\end{aligned}
$$

Thus, using $\Gamma_{I}^{2}=\mathbb{I}_{m}$, we obtain

$$
Q_{I}(y) y=\left(\left(\sum_{I \neq J=0}^{q+1} Q_{J}(y) \Gamma_{J}\right)+\left(\sum_{K=1}^{r} R_{K}(y) \Omega_{K}\right)\right) \Gamma_{I},
$$

and therefore we have verified (7.35) for $I=1, . ., q+1$. For $I=0$, it holds that $\Gamma_{0}=\mathbb{I}_{m}$ and (7.37) implies

$$
Q_{0}(y) y=\left(\left(\sum_{J=1}^{q+1} Q_{J}(y) \Gamma_{J}\right)+\left(\sum_{K=1}^{r} R_{K}(y) \Omega_{K}\right)\right) \Gamma_{0} .
$$

Consequently, we have verified (7.35) for all $I=0,1, \ldots, q+1$, and therefore we proved the second identity (7.30), as well. Hence, we proved (7.27). This concludes the verification that $\mu \circ \alpha$ provides a birational inverse of $\nabla \mathcal{V}$.

The results in section 7, holding when the condition enounced in section 6.2 is satisfied, provide the proof of the invertibility of the gradient map $\nabla_{\mathcal{V}}$ by giving its explicit birational inverse map, as discussed in section 6.4. 


\section{Examples, I: $L(q, 1), q=1, \ldots, 8$}

Whenever $P=1,2$, the various $L(q, P)$ models for which we can prove invertibility, namely those with $q=1, . ., 8$ and $P=1$ as well as those with $q=1,2,3$ and $P=2$, are conveniently presented below as suitable linear sections (also named descendants ${ }^{24}$ ) of the complete model $L(8,1)$. Note that the invertibility of these descendants is a priori by no means guaranteed by the invertibility of $L(8,1)$ (which is basically the $J_{3}^{\mathbb{Q}}$ and thus is well-known to be invertible, cfr. section 8.1); however, the general treatment given above does allow us to verify invertibility of such descendants. Furthermore, the models $L(q, P)$ with $q=1,2,3$ and $P \geqslant 3$ will then be treated with a different method in section 10 .

For the $L(q, 1)$ models (with $1 \leqslant q \leqslant 8$ ) that are not complete, so for $q=3,5,6,7$, the invertibility condition enounced in section 6.2 requires that a Clifford set of $q+1$ symmetric $\Gamma$-matrices $\left\{\Gamma_{1}, \ldots, \Gamma_{q+1}\right\}$ is contained in a larger set $\left\{\Gamma_{0}:=\mathbb{I}_{m}, \Gamma_{1}, \ldots, \Gamma_{q+1}, \Omega_{1}, \ldots, \Omega_{r}\right\}$ of symmetric matrices with certain properties. It turns out that the $\Omega_{K}$ 's (with $K=1, \ldots, r$ ) can again be taken to be $\Gamma$-matrices and that $\left\{\Gamma_{1}, \ldots, \Gamma_{q+1}, \Omega_{1}, \ldots, \Omega_{r}\right\}$ is in fact a Clifford set (of square matrices of size $m=\mathcal{D}_{q+1}$ ) for a complete model of type $L\left(q^{\prime}, 1\right)$ with $q^{\prime}>q$. More precisely:

- $L(3,1) \subset L(4,1)$ (for which $m=2^{3}=8$ ) and the corresponding Clifford sets are $\left\{\Gamma_{1}, \ldots, \Gamma_{4}\right\} \subset\left\{\Gamma_{1}, \ldots, \Gamma_{5}\right\}$, so $L(3,1)$ is a submodel of the complete model $L(4,1)$;

- $L(5,1), L(6,1)$ and $L(7,1)$ are submodels of the complete model $L(8,1)$, because they all have Clifford sets of $16 \times 16 \Gamma$-matrices which are contained in the Clifford set (still of $16 \times 16 \Gamma$-matrices) of the model $L(8,1)$ : for each of such submodels, the missing $\Gamma$-matrices w.r.t. $L(8,1)$ can be identified with the needed $\Omega$-matrices.

So, given a complete model with Clifford set $\left\{\Gamma_{1}^{\prime}, \ldots, \Gamma_{M}^{\prime}\right\}$, for any proper subset $\mathbb{S} \subset\{1, \ldots, M\}$, one obtains a descendant model (possibly with $\dot{P}>0$ ), associated to a cubic form $\mathcal{V}_{\mathbb{S}}$ defined by the $\Gamma$-matrices $\Gamma_{I}^{\prime}, I \in \mathbb{S}$, and with $\Omega_{K}$ 's defined as the $\Gamma_{K}^{\prime}$ 's with $K \in \breve{\mathbb{S}}$, where $\breve{\mathbb{S}}$ is the complementary of $\mathbb{S}$ in $\{1, \ldots, M\}$. The explicit inverse of the gradient map $\nabla \mathcal{V}_{\mathbb{S}}$ is thus obtained through the procedure treated in sections 6.2-6.7.

From the treatment of section 6 , the solution of the BPS system of the descendant model under consideration is then given by (6.54)-(6.56), and (6.66) and (6.73)-(6.75) yield the corresponding expression of the BPS black hole entropy and of the BPS attractors, respectively. We refer to section 9.5, 10.6 for examples of lower dimensional models $L(q, P)$ (namely, $L(1,2)$ and $L(1,3)$ ) which we explicitly work out in detail (see also the generalization to $L(1, P)$ models with $P \geqslant 2$ in section 10.7).

\section{$8.1 L(8,1) \equiv J_{3}^{\mathbb{O}}$}

This model is the 'largest' among the known complete models (see section 6.7): it has $q=8, P=1$, and $\mathcal{D}_{9}=16$ [53]; the number of variables is

$$
\left(1+q+2+P \cdot \mathcal{D}_{q+1}\right)_{q=8, P=1}=1+10+16=27,
$$

\footnotetext{
${ }^{24}$ But not necessarily submodels; cfr. section 11.3 .
} 
and therefore $I=0,1, \ldots, 9$, and $\alpha=1, \ldots, 16$. The model corresponds to a symmetric space, and it is related to the exceptional cubic Jordan algebra ${ }^{25} J_{3}^{\mathbb{Q}}$, whose reduced structure group is the minimally non-compact real form $E_{6(-26)}$ of $E_{6}$.

In this case, the Bekenstein-Hawking entropy and the attractor values of scalar fields of BPS extremal black holes are explicitly known; see e.g. [62]. Thus, we will not consider the solution of the BPS system in this model, but rather we will give some treatment useful to discuss some descendants from $L(8,1)$ itself (cfr. section 8.2).

We choose the $q+2=10 \Gamma$-matrices $\Gamma_{0}=\mathbb{I}_{16}, \Gamma_{1}, \ldots, \Gamma_{9}$ (of size $\left.16 \times 16\right)$ such that $\left\{\Gamma_{1}, \ldots, \Gamma_{9}\right\}$ is a Clifford set and such that the corresponding quadrics are:

$$
\begin{aligned}
& Q_{0} \equiv Q\left[\begin{array}{l}
0000 \\
0000
\end{array}\right]:=\left(y^{1}\right)^{2}+\ldots+\left(y^{8}\right)^{2}+\left(y^{9}\right)^{2}+\ldots+\left(y^{16}\right)^{2} ; \\
& Q_{1} \equiv Q\left[\begin{array}{l}
0000 \\
0001
\end{array}\right]:=\left\{\begin{array}{l}
\left(y^{1}\right)^{2}-\left(y^{2}\right)^{2}+\ldots+\left(y^{7}\right)^{2}-\left(y^{8}\right)^{2} \\
+\left(y^{9}\right)^{2}-\left(y^{10}\right)^{2}+\ldots+\left(y^{15}\right)^{2}-\left(y^{16}\right)^{2} ;
\end{array}\right. \\
& Q_{2} \equiv Q\left[\begin{array}{l}
0001 \\
1010
\end{array}\right]:=2\left(y^{1} y^{2}-y^{3} y^{4}+y^{5} y^{6}-y^{7} y^{8}-y^{9} y^{10}+y^{11} y^{12}-y^{13} y^{14}+y^{15} y^{16}\right) ; \\
& Q_{3} \equiv Q\left[\begin{array}{l}
00000 \\
0000
\end{array}\right]:=2\left(y^{1} y^{4}+y^{2} y^{3}+y^{5} y^{8}+y^{6} y^{7}+y^{9} y^{12}+y^{10} y^{11}+y^{13} y^{16}+y^{14} y^{15}\right) ; \\
& Q_{4} \equiv Q\left[\begin{array}{l}
0101 \\
0101
\end{array}\right]:=2\left(y^{1} y^{6}-y^{2} y^{5}+y^{3} y^{8}-y^{4} y^{7}+y^{9} y^{14}-y^{10} y^{13}+y^{11} y^{16}-y^{12} y^{15}\right) ; \\
& Q_{5} \equiv Q\left[\begin{array}{l}
0111 \\
0110
\end{array}\right]:=2\left(y^{1} y^{8}+y^{2} y^{7}-y^{3} y^{6}-y^{4} y^{5}+y^{9} y^{16}+y^{10} y^{15}-y^{11} y^{14}-y^{12} y^{13}\right) ; \\
& Q_{6} \equiv Q\left[\begin{array}{l}
10011 \\
0010
\end{array}\right]:=2\left(y^{1} y^{10}+y^{2} y^{9}-y^{3} y^{12}-y^{4} y^{11}+y^{5} y^{14}+y^{6} y^{13}-y^{7} y^{16}-y^{8} y^{15}\right) ; \\
& Q_{7} \equiv Q\left[\begin{array}{l}
1011 \\
1101
\end{array}\right]:=2\left(y^{1} y^{12}-y^{2} y^{11}+y^{3} y^{10}-y^{4} y^{9}-y^{5} y^{16}+y^{6} y^{15}-y^{7} y^{14}+y^{8} y^{13}\right) ; \\
& Q_{8} \equiv Q\left[\begin{array}{l}
1101 \\
1110
\end{array}\right]:=2\left(y^{1} y^{14}+y^{2} y^{13}-y^{3} y^{16}-y^{4} y^{15}-y^{5} y^{10}-y^{6} y^{9}+y^{7} y^{12}+y^{8} y^{11}\right) ; \\
& Q_{9} \equiv Q\left[\begin{array}{l}
1111 \\
1001
\end{array}\right]:=2\left(y^{1} y^{16}-y^{2} y^{15}+y^{3} y^{14}-y^{4} y^{13}+y^{5} y^{12}-y^{6} y^{11}+y^{7} y^{10}-y^{8} y^{9}\right) .
\end{aligned}
$$

The cubic form of this model is

$$
\mathcal{V}_{L(8,1)}:=s q(x)+\sum_{I=0}^{9} x^{I} Q_{I}(y),
$$

where

$$
q(x):=-\left(x^{0}\right)^{2}+q_{9}(x)=-\left(x^{0}\right)^{2}+\left(x^{1}\right)^{2}+\left(x^{2}\right)^{2}+\ldots+\left(x^{9}\right)^{2} .
$$

Since the quadratic forms $Q_{0}, \ldots, Q_{9}$ satisfy the Lorentzian quadratic relation

$$
-Q_{0}(y)^{2}+Q_{1}(y)^{2}+\ldots+Q_{9}(y)^{2}=0,
$$

the $L(8,1)$ model satisfies the invertibility condition of section 6.2 with $r=0$ (so no extra $\Omega_{K}$ are needed): in fact, as mentioned above, $L(8,1)$ is a complete model. Thus, the gradient map $\nabla_{\mathcal{V}_{L(8,1)}}$ of $\mathcal{V}_{L(8,1)}$ is invertible and the inverse map $\mu$ is given by homogeneous polynomials of degree two. It holds that

$$
\mathbb{R}_{\xi}^{27} \stackrel{\nabla \mathcal{V}_{L(8,1)}}{\longrightarrow} \mathbb{R}_{z}^{27} \stackrel{\mu}{\longrightarrow} \mathbb{R}_{\xi}^{27},
$$

\footnotetext{
${ }^{25}$ In fact, one could in principle write down an explicit $3 \times 3$ Hermitian matrix $M$ with octonionic components such that $\mathcal{V}=-\mathcal{N}(M)$, where $\mathcal{N}$ is the cubic norm (generalizing the determinant) of $M$, but we refrain from doing so; see [89].
} 
with

$$
\mu \circ \nabla_{\mathcal{V}_{L(8,1)}}(\xi)=4 \mathcal{V}_{L(8,1)}(\xi) \xi
$$

The gradient map

$$
\nabla \mathcal{V}_{L(8,1)}: \mathbb{R}^{27} \longrightarrow \mathbb{R}^{27}, \quad \xi \longmapsto{ }^{T}\left(\mathcal{V}_{s}(\xi), \mathcal{V}_{I}(\xi), \mathcal{V}_{\alpha}(\xi)\right)
$$

can be identified with the adjoint map

$$
J_{3}^{\mathbb{Q}} \longrightarrow J_{3}^{\mathbb{Q}}, \quad M \longmapsto M^{\sharp}
$$

here $M^{\sharp}$ is the adjoint matrix of $M \in J_{3}^{\mathbb{Q}}$. This adjoint map \# is (birationally) invertible, with inverse given by the map $M^{\sharp} \mapsto\left(M^{\sharp}\right)^{\sharp}$, which is thus essentially the map $\mu$.

\subsection{Some submodels of $L(8,1)$}

\subsubsection{L $L(7,1)$}

$L(7,1)$ is a submodel of $L(8,1)$, and it corresponds to a homogeneous non-symmetric (sub)manifold (of the symmetric scalar manifold pertaining to $L(8,1)$ ). Indeed, by setting $x^{9}=0$ in the $L(8,1)$ model treated in section 8.1 , one has $1+(10-1)+16=26$ variables. The $\Gamma$-matrices involved are $\Gamma_{0}=\mathbb{I}_{16}$ and the Clifford set, now with only 8 matrices, namely $\left\{\Gamma_{1}, \ldots, \Gamma_{8}\right\}$, still of size $\mathcal{D}_{9}=\mathcal{D}_{8}=16$. The $x^{9}=0$ restriction of the Lorentzian quadratic form $q(x)(8.4)$ is the corresponding Lorentzian form in $x^{0}, \ldots, x^{8}$ :

$$
q(x):=-\left(x^{0}\right)^{2}+q_{8}(x)=-\left(x^{0}\right)^{2}+\left(x^{1}\right)^{2}+\left(x^{2}\right)^{2}+\ldots+\left(x^{8}\right)^{2} .
$$

Therefore, $\mathcal{V}_{L(8,1,0)}$ restricts to the cubic form $\mathcal{V}_{L(7,1)}$ of the $L(7,1)$ model: $L(7,1)$ can be regarded as $L(8,1)$ with one linear constraint:

$$
\begin{aligned}
& L(7,1)=\left.L(8,1)\right|_{x^{9}=0} ; \\
& \mathcal{V}_{L(7,1)}=\left.\mathcal{V}_{L(8,1)}\right|_{x^{9}=0}
\end{aligned}
$$

As discussed in [117], it should be remarked that the invertibility of the gradient map of $\mathcal{V}_{L(8,1)}$ would, in general, not guarantee the invertibility of its restriction to a linear subspace of $\mathbb{R}^{27}$. However, the invertibility condition enounced in section 6.2 is satisfied, once we define

$$
\Omega_{1}:=\Gamma_{9}, \quad \text { so } \quad R_{1}(y)=Q_{9}(y), \quad r:=1 .
$$

In fact, $\Omega_{1}$ anti-commutes with all matrices of the Clifford set $\left\{\Gamma_{1}, \ldots, \Gamma_{8}\right\}$, because it is originally part of the Clifford set $\left\{\Gamma_{1}, \ldots, \Gamma_{9}\right\}$ of $L(8,1)$. The quadratic form $R_{1}(y)$ defined by $\Omega_{1}=\Gamma_{9}$ is of course $R_{1}(y)=Q_{9}(y)$ of (8.2), so that the required Lorentzian identity (6.8) holds true: it is nothing but (8.5).

Since $r=1$, the inverse of the gradient map $\nabla \mathcal{V}_{L(7,1)}$ is given as a composition of two maps, namely (cfr. (4.41)):

$$
\mathbb{R}_{\xi}^{1+9+16} \stackrel{\nabla_{\mathcal{V}_{L(7,1)}}^{\longrightarrow}}{\longrightarrow} \mathbb{R}_{z}^{1+9+16} \stackrel{\alpha}{\longrightarrow} \mathbb{R}_{(t, u, v, w)}^{1+9+16+1} \stackrel{\mu}{\longrightarrow} \mathbb{R}_{\xi}^{1+9+16}
$$


The map $\alpha$, as in (6.14), is given by:

$$
\alpha\left(z_{1}, \ldots, z_{26}\right):={ }^{T}\left(z_{1}^{2}, z_{1} z_{2}, \ldots, z_{1} z_{26}, R_{1}(z)\right),
$$

where the quadratic form $R_{1}(z)$ is obtained by substituting $y^{\alpha}:=z_{\alpha+10}$ in $Q_{9}$ :

$$
R_{1}(z):=2\left(z_{11} z_{26}-z_{12} z_{25}+z_{13} z_{24}-z_{14} z_{23}+z_{15} z_{22}-z_{16} z_{21}+z_{17} z_{20}-z_{18} z_{19}\right) .
$$

The map $\mu$ (6.17) has $1+9+16$ components which are homogeneous polynomials of degree 2 in the 27 variables $t, u_{0}, \ldots, u_{8}, v_{1}, \ldots, v_{16}, w$. Since

$$
\mu \circ \alpha \circ \nabla_{\mathcal{V}_{L(7,1)}}(\xi)=4 q(x)^{2} \mathcal{V}_{L(7,1)}(\xi) \xi
$$

where $q(x)$ is given by (8.9), the (birational) inverse of the gradient map $\nabla_{\mathcal{V}_{L(7,1)}}$ is the map $\mu \circ \alpha$, which is a homogeneous polynomial map of degree four.

\subsubsection{L $L(6,1)$}

A similar treatment can be given for $L(6,1)$, corresponding to an homogeneous nonsymmetric (sub)manifold (of the symmetric scalar manifold pertaining to $L(8,1)$ ): $L(6,1$ ) can be regarded as $L(8,1)$ with two linear constraints:

$$
\begin{aligned}
& L(6,1)=\left.L(8,1)\right|_{x^{8}=x^{9}=0} ; \\
& \mathcal{V}_{L(6,1)}=\left.\mathcal{V}_{L(8,1)}\right|_{x^{8}=x^{9}=0}
\end{aligned}
$$

Therefore, from the invertibility condition enounced in section 6.2 , the model $L(6,1)$ is invertible, because one can take

$$
\Omega_{1}=\Gamma_{9}, \quad \Omega_{2}=\Gamma_{8}, \quad \text { so } \quad R_{1}(y)=Q_{9}(y), \quad R_{2}(y)=Q_{8}(y), \quad r=2 .
$$

The $x^{8}=x^{9}=0$ restriction of the Lorentzian quadratic form $q(x)(8.4)$ is the corresponding Lorentzian form in $x^{0}, \ldots, x^{7}$ :

$$
q(x):=-\left(x^{0}\right)^{2}+q_{7}(x)=-\left(x^{0}\right)^{2}+\left(x^{1}\right)^{2}+\left(x^{2}\right)^{2}+\ldots+\left(x^{7}\right)^{2} .
$$

Since $r=2$, the inverse of the gradient map $\nabla_{\mathcal{V}_{L(6,1)}}$ is given as a composition of two maps, namely (cfr. (4.41)):

$$
\mathbb{R}_{\xi}^{1+8+16} \stackrel{\nabla_{\mathcal{V}_{L(6,1)}}^{\longrightarrow}}{\longrightarrow} \mathbb{R}_{z}^{1+8+16} \stackrel{\alpha}{\longrightarrow} \mathbb{R}_{(t, u, v, w)}^{1+8+16+2} \stackrel{\mu}{\longrightarrow} \mathbb{R}_{\xi}^{1+8+16}
$$

The map $\alpha$, as in (6.14), is given by:

$$
\alpha\left(z_{1}, \ldots, z_{25}\right):={ }^{T}\left(z_{1}^{2}, z_{1} z_{2}, \ldots, z_{1} z_{25}, R_{1}(z), R_{2}(z)\right),
$$

where the quadratic forms $R_{K}(z)$ 's $(K=1,2)$ depend only on the last 16 variables $z_{10}, \ldots, z_{25}$ and they are obtained by substituting $y^{\alpha}:=z_{\alpha+9}$ in $Q_{9}$ and $Q_{8}$ respectively:

$$
\begin{aligned}
& R_{1}(z):=2\left(z_{10} z_{25}-z_{11} z_{24}+z_{12} z_{23}-z_{13} z_{22}+z_{14} z_{21}-z_{15} z_{20}+z_{16} z_{19}-z_{17} z_{18}\right) \\
& R_{2}(z):=2\left(z_{10} z_{23}+z_{11} z_{22}-z_{12} z_{25}-z_{13} z_{24}-z_{14} z_{19}-z_{15} z_{18}+z_{16} z_{21}+z_{17} z_{20}\right) .
\end{aligned}
$$


The map $\mu$ (6.17) has $1+8+16$ components which are homogeneous polynomials of degree 2 in the 27 variables $t, u_{0}, \ldots, u_{7}, v_{1}, \ldots, v_{16}, w_{1}, w_{2}$. Since

$$
\mu \circ \alpha \circ \nabla_{\mathcal{V}_{L(6,1)}}(\xi)=4 q(x)^{2} \mathcal{V}_{L(6,1)}(\xi) \xi,
$$

where $q(x)$ is given by (8.20), the (birational) inverse of the gradient map $\nabla_{\mathcal{V}_{L(6,1)}}$ is the map $\mu \circ \alpha$, which is a homogeneous polynomial map of degree four.

\subsubsection{L $L(5,1)$}

One can take one step further (since the $\Gamma$-matrices still have size $16=\mathcal{D}_{9}=\mathcal{D}_{8}=\mathcal{D}_{7}=\mathcal{D}_{6}$ ), and consider $L(5,1)$, corresponding to a homogeneous non-symmetric (sub)manifold (of the symmetric scalar manifold pertaining to $L(8,1)): L(5,1)$ can be regarded as $L(8,1)$ with three linear constraints:

$$
\begin{aligned}
& L(5,1)=\left.L(8,1)\right|_{x^{7}=x^{8}=x^{9}=0} \\
& \mathcal{V}_{L(5,1)}=\left.\mathcal{V}_{L(8,1)}\right|_{x^{7}=x^{8}=x^{9}=0}
\end{aligned}
$$

One can thus take

$$
\Omega_{1}=\Gamma_{9}, \quad \Omega_{2}=\Gamma_{8}, \quad \Omega_{3}=\Gamma_{7} \quad \text { so } \quad R_{1}(y)=Q_{9}(y), \quad R_{2}(y)=Q_{8}(y), \quad R_{3}(y)=Q_{7}(y),
$$

and $r=3$, in order to satisfy the invertibility condition of section 6.2 . The $x^{7}=x^{8}=x^{9}=0$ restriction of the Lorentzian quadratic form $q(x)(8.4)$ is the corresponding Lorentzian form in $x^{0}, \ldots, x^{6}$ :

$$
q(x):=-\left(x^{0}\right)^{2}+q_{6}(x)=-\left(x^{0}\right)^{2}+\left(x^{1}\right)^{2}+\left(x^{2}\right)^{2}+\ldots+\left(x^{6}\right)^{2} .
$$

Since $r=3$, the inverse of the gradient map $\nabla_{\mathcal{V}_{L(5,1)}}$ is given as a composition of two maps, namely (cfr. (4.41)):

$$
\mathbb{R}_{\xi}^{1+7+16} \stackrel{\nabla_{\mathcal{V}_{L(5,1)}}^{\longrightarrow}}{\longrightarrow} \mathbb{R}_{z}^{1+7+16} \stackrel{\alpha}{\longrightarrow} \mathbb{R}_{(t, u, v, w)}^{1+7+16+3} \stackrel{\mu}{\longrightarrow} \mathbb{R}_{\xi}^{1+7+16}
$$

The map $\alpha$, as in (6.14), is given by:

$$
\alpha\left(z_{1}, \ldots, z_{24}\right):={ }^{T}\left(z_{1}^{2}, z_{1} z_{2}, \ldots, z_{1} z_{24}, R_{1}(z), R_{2}(z), R_{3}(z)\right),
$$

where the quadratic forms $R_{K}(z)$ 's $(K=1,2,3)$ depend only on the last 16 variables $z_{9}, \ldots, z_{24}$ and they are obtained by substituting $y^{\alpha}:=z_{\alpha+8}$ in $Q_{9}, Q_{8}, Q_{7}$ respectively:

$$
\begin{aligned}
& R_{1}(z):=2\left(z_{9} z_{24}-z_{10} z_{23}+z_{11} z_{22}-z_{12} z_{21}+z_{13} z_{20}-z_{14} z_{19}+z_{15} z_{18}-z_{16} z_{17}\right) \\
& R_{2}(z):=2\left(z_{9} z_{22}+z_{10} z_{21}-z_{11} z_{24}-z_{12} z_{23}-z_{13} z_{18}-z_{14} z_{17}+z_{15} z_{20}+z_{16} z_{19}\right) \\
& R_{3}(z):=2\left(z_{9} z_{20}-z_{10} z_{19}+z_{11} z_{18}-z_{12} z_{17}-z_{13} z_{24}+z_{14} z_{23}-z_{15} z_{22}+z_{16} z_{21}\right) .
\end{aligned}
$$

The map $\mu$ (6.17) has $1+7+16$ components which are homogeneous polynomials of degree 2 in the 27 variables $t, u_{0}, \ldots, u_{6}, v_{1}, \ldots, v_{16}, w_{1}, w_{2}, w_{3}$. Since

$$
\mu \circ \alpha \circ \nabla_{\mathcal{V}_{L(5,1)}}(\xi)=4 q(x)^{2} \mathcal{V}_{L(5,1)}(\xi) \xi,
$$

where $q(x)$ is given by (8.29), the (birational) inverse of the gradient map $\nabla \mathcal{V}_{L(5,1)}$ is the map $\mu \circ \alpha$, which is a homogeneous polynomial map of degree four. 


\subsection{4 $L(4,1) \equiv J_{3}^{\mathbb{H}}$}

The model $L(4,1)$ corresponds to a symmetric space, and it is related to the simple cubic Jordan algebra over the quaternions, $J_{3}^{\mathbb{H}}$. In this case, the Bekenstein-Hawking entropy and the attractor values of scalar fields of extremal BPS black holes are explicitly known; see e.g. [62]. In the present treatment, we will highlight its relation to the complete 'parent' model $L(8,1)$.

It should be remarked that going one step further from $L(5,1)$ and imposing $x^{6}=x^{7}=$ $x^{8}=x^{9}=0$ does not yield to the $L(4,1)$ model, since the $\Gamma$-matrices in such a model have size $\mathcal{D}_{5}=8$, and not size 16 as the ones of $L(5,1)$. Fortunately, our choice of $\Gamma$-matrices in (8.2) is such that taking the upper left $8 \times 8$ block of $\Gamma_{0}=\mathbb{I}_{16}, \Gamma_{1}, \ldots, \Gamma_{5}$, one obtains $\mathbb{I}_{8}$ and the 5 other $\Gamma$-matrices of size 8 that are easily verified to be again a Clifford set; taking similar blocks of $\Gamma_{6}, \ldots, \Gamma_{9}$ one finds the zero matrix of size 8 . In other words, the restriction of the quadrics $Q_{i}(y)$ from (8.2) to the subspace $y^{9}=\ldots=y^{16}=0$ yields to the quadrics of $L(4,1)$. To be explicit:

$$
\begin{aligned}
& \bar{Q}_{0} \equiv\left(Q_{0}\right)_{\mid y^{9}=\ldots=y^{16}=0}=Q\left[\begin{array}{l}
000 \\
000
\end{array}\right]:=\left(y^{1}\right)^{2}+\left(y^{2}\right)^{2}+\ldots+\left(y^{7}\right)^{2}+\left(y^{8}\right)^{2} ; \\
& \bar{Q}_{1} \equiv\left(Q_{1}\right)_{\mid y^{9}=\ldots=y^{16}=0}=Q\left[\begin{array}{l}
000 \\
001
\end{array}\right]:=\left(y^{1}\right)^{2}-\left(y^{2}\right)^{2}+\ldots+\left(y^{7}\right)^{2}-\left(y^{8}\right)^{2} ; \\
& \bar{Q}_{2} \equiv\left(Q_{2}\right)_{\mid y^{9}=\ldots=y^{16}=0}=Q\left[\begin{array}{l}
001 \\
010
\end{array}\right]:=2\left(y^{1} y^{2}-y^{3} y^{4}+y^{5} y^{6}-y^{7} y^{8}\right) ; \\
& \bar{Q}_{3} \equiv\left(Q_{3}\right)_{\mid y^{9}=\ldots=y^{16}=0}=Q\left[\begin{array}{l}
011 \\
000
\end{array}\right]:=2\left(y^{1} y^{4}+y^{2} y^{3}+y^{5} y^{8}+y^{6} y^{7}\right) ; \\
& \bar{Q}_{4} \equiv\left(Q_{4}\right)_{\mid y^{9}=\ldots=y^{16}=0}=Q\left[\begin{array}{l}
101 \\
101
\end{array}\right]:=2\left(y^{1} y^{6}-y^{2} y^{5}+y^{3} y^{8}-y^{4} y^{7}\right) ; \\
& \bar{Q}_{5} \equiv\left(Q_{5}\right)_{\mid y^{9}=\ldots=y^{16}=0}=Q\left[\begin{array}{l}
111 \\
110
\end{array}\right]:=2\left(y^{1} y^{8}+y^{2} y^{7}-y^{3} y^{6}-y^{4} y^{5}\right) ; \\
& \left(Q_{6}\right)_{\mid y^{9}=\ldots=y^{16}=0}=0 ; \\
& \left(Q_{7}\right)_{\mid y^{9}=\ldots=y^{16}=0}=0 ; \\
& \left(Q_{8}\right)_{\mid y^{9}=\ldots=y^{16}=0}=0 ; \\
& \left(Q_{9}\right)_{\mid y^{9}=\ldots=y^{16}=0}=0 .
\end{aligned}
$$

The observant reader will have noticed that, in order to obtain the characteristics in (8.36), we omitted the first column from the characteristics in the first six quadrics in (8.2). It is easy to check that the sum of any two distinct non-zero characteristics is odd, hence the $\Gamma$-matrices of size 8 corresponding to the last 5 quadrics compose a Clifford set.

Next, we observe that if we put $y^{9}=\ldots=y^{16}=0$ in the Lorentzian identity (8.5) between $Q_{0}(y), \ldots, Q_{9}(y)$, we obtain the following Lorentzian identity between the 'restricted' $Q_{0}, \ldots, Q_{5}$, denoted by $\bar{Q}_{0}(\bar{y}), \ldots, \bar{Q}_{5}(\bar{y})\left(\right.$ with $\left.\bar{y}=\left(y^{1}, \ldots, y^{8}\right)\right)$ :

$$
-\bar{Q}_{0}(\bar{y})^{2}+\bar{Q}_{1}(\bar{y})^{2}+\ldots+\bar{Q}_{5}(\bar{y})^{2}=0 .
$$

This implies that $L(4,1)$ is a complete model, that is $r=0$ in section 6.2 , since no extra $8 \times 8$ matrices $\Omega_{K}$ 's are needed.

To summarize, the complete model $L(4,1)$ can be regarded as the complete model $L(8,1)$ with twelve linear constraints:

$$
\begin{aligned}
& L(4,1)=\left.L(8,1)\right|_{x^{6}=\ldots=x^{9}=0, y^{9}=\ldots=y^{16}=0} \\
& \mathcal{V}_{L(4,1)}=\left.\mathcal{V}_{L(8,1)}\right|_{x^{6}=\ldots=x^{9}=0, y^{9}=\ldots=y^{16}=0}
\end{aligned} .
$$


Since the model is complete, so $r=0$, the map $\alpha$ is not needed and the inverse of the gradient map is now simply the map $\mu$ from (6.17):

$$
\mathbb{R}_{\xi}^{15} \stackrel{\nabla \mathcal{V}_{L(4,1)}}{\longrightarrow} \mathbb{R}_{z}^{15} \stackrel{\mu}{\longrightarrow} \mathbb{R}_{\xi}^{15}
$$

with

$$
\mu \circ \nabla \mathcal{V}_{L(4,1)}(\xi)=4 \mathcal{V}_{L(4,1)}(\xi) \xi
$$

The gradient map

$$
\nabla \mathcal{V}_{L(4,1)}: \mathbb{R}^{15} \longrightarrow \mathbb{R}^{15}, \quad \xi \longmapsto{ }^{T}\left(\mathcal{V}_{s}(\xi), \mathcal{V}_{I}(\xi), \mathcal{V}_{\alpha}(\xi)\right)
$$

can be identified with the adjoint map

$$
J_{3}^{\mathbb{H}} \longrightarrow J_{3}^{\mathbb{H}}, \quad M \longmapsto M^{\sharp}
$$

here $M^{\sharp}$ is the adjoint matrix of $M \in J_{3}^{\mathbb{H}}$. This adjoint map \# is (birationally) invertible, with inverse given by the map $M^{\sharp} \mapsto\left(M^{\sharp}\right)^{\sharp}$, which is thus essentially the map $\mu$.

The cubic norm of $\boldsymbol{J}_{\mathbf{3}}^{\mathbb{H}}$. It is worth making more explicit the relation between the complete model $L(4,1)$ and the Euclidean simple cubic Jordan algebra $J_{3}^{\mathbb{H}}$. Let $\mathbf{i}, \mathbf{j}$ and $\mathbf{k}$ denote the imaginary units of $\mathbb{H}$, with standard multiplication rules of $\mathbb{H}$ :

$$
\left.\begin{array}{l}
\mathbf{i j}=-\mathbf{j i}=\mathbf{k} ; \\
\mathbf{j k}=-\mathbf{k j}=\mathbf{i} ; \\
\mathbf{k i}=-\mathbf{i k}=\mathbf{j}
\end{array}\right\} \Longleftrightarrow \mathbf{i}^{2}=\mathbf{j}^{2}=\mathbf{k}^{2}=\mathbf{i j k}=-1
$$

Then, one can define the following matrix $\mathcal{M}$ belonging to $J_{3}^{\mathbb{H}}$ :

$$
J_{3}^{\mathbb{H}} \ni \mathcal{M}=\mathcal{M}^{\mathbb{H}}:=\left(\begin{array}{ccc}
a & \mathbf{z} & \overline{\mathbf{y}} \\
\overline{\mathbf{z}} & b & \mathbf{x} \\
\mathbf{y} & \overline{\mathbf{x}} & c
\end{array}\right)
$$

with

$$
\begin{array}{rlrl}
a:=x^{0}-x^{1} \in \mathbb{R}, & \mathbf{x}:=y^{2}-\mathbf{i} y^{4}+\mathbf{j} y^{6}-\mathbf{k} y^{8} \in \mathbb{H} ; \\
b:=x^{0}+x^{1} \in \mathbb{R}, & \mathbf{y}:=y^{1}-\mathbf{i} y^{3}-\mathbf{j} y^{5}-\mathbf{k} y^{7} \in \mathbb{H} ; \\
c:= & s \in \mathbb{R}, & \mathbf{z}:=-x^{2}-\mathbf{i} x^{3}+\mathbf{j} x^{4}-\mathbf{k} x^{5} \in \mathbb{H} .
\end{array}
$$

The cubic norm $\mathcal{N}(\mathcal{M})$ of $\mathcal{M}$ is defined as

$$
\mathcal{N}(\mathcal{M}):=a b c-a \mathbf{x} \overline{\mathbf{x}}-b \mathbf{y} \overline{\mathbf{y}}-c \mathbf{z} \overline{\mathbf{z}}+(\mathbf{x y}) \mathbf{z}+\overline{\mathbf{z}}(\overline{\mathbf{y} x})
$$

With this choice of coefficients, one can check

$$
\mathcal{V}_{L(4,1)}=-\mathcal{N}(\mathcal{M})
$$




\subsubsection{L $L(3,1)$}

This model, which also has size $\mathcal{D}_{4}=8$, is found by setting $x^{5}=0$ in $L(4,1)$ and taking $\Omega_{1}$ to be the $\Gamma$-matrix of $\bar{Q}_{5}$, so $r=1$ :

$$
\begin{aligned}
& L(3,1)=\left.L(8,1)\right|_{x^{5}=\ldots=x^{9}=0, y^{9}=\ldots=y^{16}=0}=\left.L(4,1)\right|_{x^{5}=0} \\
& \mathcal{V}_{L(3,1)}=\left.\mathcal{V}_{L(8,1)}\right|_{x^{5}=\ldots=x^{9}=0, y^{9}=\ldots=y^{16}=0}=\left.\mathcal{V}_{L(4,1)}\right|_{x^{5}=0}
\end{aligned}
$$

Thus, $L(3,1)$ can be regarded as the complete model $L(8,1)$ with thirteen linear constraints, or equivalently as the complete model $L(4,1)$ with one linear constraint. In fact, $L(3,1)$ corresponds to a homogeneous non-symmetric (sub)manifold (of the symmetric scalar manifolds pertaining to $L(8,1)$ and $L(4,1))$.

In order to satisfy the invertibility condition of section 6.2 one can thus take

$$
\Omega_{1}=\bar{\Gamma}_{5} \quad \text { so } \quad R_{1}(y)=\bar{Q}_{5}(y), \quad r=1 .
$$

The $x^{5}=x^{6}=x^{7}=x^{8}=x^{9}=0$ restriction of the Lorentzian quadratic form $q(x)$ (8.4) is the corresponding Lorentzian form in $x^{0}, \ldots, x^{4}$ :

$$
q(x):=-\left(x^{0}\right)^{2}+q_{4}(x)=-\left(x^{0}\right)^{2}+\left(x^{1}\right)^{2}+\left(x^{2}\right)^{2}+\left(x^{3}\right)^{2}+\left(x^{4}\right)^{2} .
$$

Since $r=1$, the inverse of the gradient map $\nabla_{\mathcal{V}_{L(3,1)}}$ is given as a composition of two maps, namely (cfr. (4.41)):

$$
\mathbb{R}_{\xi}^{1+5+8} \stackrel{\nabla_{\mathcal{V}_{L(7,1)}}^{\longrightarrow}}{\longrightarrow} \mathbb{R}_{z}^{1+5+8} \stackrel{\alpha}{\longrightarrow} \mathbb{R}_{(t, u, v, w)}^{1+5+8+1} \stackrel{\mu}{\longrightarrow} \mathbb{R}_{\xi}^{1+5+8} .
$$

The map $\alpha$, as in (6.14), is given by:

$$
\alpha\left(z_{1}, \ldots, z_{14}\right):={ }^{T}\left(z_{1}^{2}, z_{1} z_{2}, \ldots, z_{1} z_{14}, R_{1}(z)\right),
$$

where the quadratic form $R_{1}(z)$ is defined by $\bar{Q}_{5}$ and depends only on the last 8 variables $z_{7}, \ldots, z_{14}$, obtained by substituting $y^{\alpha}=z_{\alpha+6}$ in $\bar{Q}_{5}(y)$ :

$$
R_{1}(z):=2\left(z_{7} z_{14}+z_{8} z_{13}-z_{9} z_{12}-z_{10} z_{11}\right) .
$$

The map $\mu$ (6.17) has $1+5+8$ components which are homogeneous polynomials of degree 2 in the 15 variables $t, u_{0}, \ldots, u_{4}, v_{1}, \ldots, v_{8}, w$. Since

$$
\mu \circ \alpha \circ \nabla_{\mathcal{V}_{L(3,1)}}(\xi)=4 q(x)^{2} \mathcal{V}_{L(3,1)}(\xi) \xi,
$$

where $q(x)$ is given by (8.51), the (birational) inverse of the gradient map $\nabla_{\mathcal{V}_{L(3,1)}}$ is the map $\mu \circ \alpha$, which is a homogeneous polynomial map of degree four.

\subsection{6 $L(2,1) \equiv J_{3}^{\mathbb{C}}$}

The model $L(2,1)$ corresponds to a symmetric space, and it is related to the simple cubic Jordan algebra over the complex numbers, $J_{3}^{\mathbb{C}}$. In this case, the Bekenstein-Hawking entropy and the attractor values of scalar fields of extremal BPS black holes are explicitly known; 
see e.g. [62]. In the present treatment, we will highlight its relation to the complete 'parent' models $L(8,1)$ and $L(4,1)$.

This model is complete: has $q=2, P=1$, and $\mathcal{D}_{3}=4$ [53]; thus, the number of variables is

$$
\left(1+q+2+P \cdot D_{q+1}\right)_{q=2, P=1}=1+4+4=9 .
$$

This complete model can be regarded as a linearly constrained $L(4,1)$ model, and thus as a linearly constrained $L(8,1)$ model, as well. We substitute $\left(y^{1}, y^{2}, y^{3}, y^{4}\right)=\left(y^{1}, y^{2}, 0,0\right)$ in the quadratic forms from the $L(4,1)$ model in (8.36), then one obtains the following quadratic forms with associated $\Gamma$-matrices:

$$
\begin{array}{ll}
\Gamma_{0}=\mathbb{I}_{4}, & Q_{0} \equiv Q\left[\begin{array}{l}
00 \\
00
\end{array}\right]=\left(y^{1}\right)^{2}+\left(y^{2}\right)^{2}+\left(y^{3}\right)^{2}+\left(y^{4}\right)^{2} ; \\
\Gamma_{1}=\sigma_{3} \otimes \mathbb{I}_{2}, & Q_{1} \equiv Q\left[\begin{array}{l}
00 \\
01
\end{array}\right]=\left(y^{1}\right)^{2}-\left(y^{2}\right)^{2}+\left(y^{3}\right)^{2}-\left(y^{4}\right)^{2} ; \\
\Gamma_{2}=\sigma_{1} \otimes \sigma_{3}, & Q_{2} \equiv Q\left[\begin{array}{l}
11 \\
10
\end{array}=2\left(y^{1} y^{2}-y^{3} y^{4}\right) ;\right. \\
\Gamma_{3}=\sigma_{1} \otimes \sigma_{1}, & Q_{3} \equiv Q\left[\begin{array}{l}
11 \\
00
\end{array}\right]=2\left(y^{1} y^{4}+y^{2} y^{3}\right) ;
\end{array}
$$

notice that

$$
\left(Q_{4}\right)_{\mid y^{5}=\ldots=y^{16}=0}=\left(Q_{5}\right)_{\mid y^{5}=\ldots=y^{16}=0}=0 .
$$

Thus, $L(2,1)$ can be regarded as $L(8,1)$ with $6+12=18$ linear constraints, or equivalently as $L(4,1)$ with $2+4=6$ linear constraints:

$$
\begin{aligned}
& L(2,1)=\left.L(8,1)\right|_{x^{4}=\ldots=x^{9}=0, y^{5}=\ldots=y^{16}=0}=\left.L(4,1)\right|_{x^{4}=x^{5}=0, y^{5}=\ldots=y^{8}=0} \\
& \mathcal{V}_{L(2,1)}=\left.\mathcal{V}_{L(8,1)}\right|_{x^{4}=\ldots=x^{9}=0, y^{5}=\ldots=y^{16}=0}=\left.\mathcal{V}_{L(4,1)}\right|_{x^{4}=x^{5}=0, y^{5}=\ldots=y^{8}=0}
\end{aligned}
$$

The set $\left\{\Gamma_{1}, \Gamma_{2}, \Gamma_{3}\right\}$ is a Clifford set, and the associated quadrics satisfy the Lorentzian quadratic relation

$$
-Q_{0}^{2}+Q_{2}^{1}+Q_{2}^{2}+Q_{3}^{2}=0
$$

Thus, the cubic form reads

$$
\mathcal{V}_{L(2,1)}\left(s, x^{0}, \ldots, x^{3}, y^{1}, \ldots, y^{4}\right):=s q(x)+\sum_{I=0}^{3} x^{I} Q_{I}(y)
$$

where the $x^{4}=x^{5}=x^{6}=x^{7}=x^{8}=x^{9}=0$ restriction of the Lorentzian quadratic form $q(x)$ (8.4) is the corresponding Lorentzian form in $x^{0}, \ldots, x^{3}$ :

$$
q(x):=-\left(x^{0}\right)^{2}+q_{3}(x)=-\left(x^{0}\right)^{2}+\left(x^{1}\right)^{2}+\left(x^{2}\right)^{2}+\left(x^{3}\right)^{2} .
$$

The invertibility condition enounced in section 6.2 yields that the gradient map $\nabla_{\mathcal{V}}$ is invertible, with $r=0$. Thus, the map $\alpha$ can be identified with the identity map and the inverse map of the gradient map $\nabla_{\mathcal{V}_{L(2,1)}}$ is simply $\mu$, which is a polynomial map of degree 2:

$$
\mathbb{R}_{\xi}^{9} \stackrel{\nabla \mathcal{V}_{L(2,1)}}{\longrightarrow} \mathbb{R}_{z}^{9} \stackrel{\mu}{\longrightarrow} \mathbb{R}_{\xi}^{9}
$$

with

$$
\mu \circ \nabla_{\mathcal{V}_{L(2,1)}}(\xi)=4 \mathcal{V}_{L(2,1)}(\xi) \xi
$$


The gradient map

$$
\nabla \mathcal{V}_{L(2,1)}: \mathbb{R}^{9} \longrightarrow \mathbb{R}^{9}, \quad \xi \longmapsto{ }^{T}\left(\mathcal{V}_{s}(\xi), \mathcal{V}_{I}(\xi), \mathcal{V}_{\alpha}(\xi)\right)
$$

can be identified with the adjoint map

$$
J_{3}^{\mathbb{C}} \longrightarrow J_{3}^{\mathbb{C}}, \quad M \longmapsto M^{\sharp}
$$

here $M^{\sharp}$ is the adjoint matrix of $M \in J_{3}^{\mathbb{C}}$. This adjoint map \# is (birationally) invertible, with inverse given by the map $M^{\sharp} \mapsto\left(M^{\sharp}\right)^{\sharp}$, which is thus essentially the map $\mu$.

The determinant of $\boldsymbol{J}_{\mathbf{3}}^{\mathbb{C}}$. Again, it is worth making more explicit the relation between the complete model $L(2,1)$ and the Euclidean simple cubic Jordan algebra $J_{3}^{\mathbb{C}}$. If $\mathbf{i}$ denotes the imaginary unit of $\mathbb{C}$, then to $\xi={ }^{T}\left(s, x^{0}, x^{1}, x^{2}, x^{3}, y^{1}, y^{2}, y^{3}, y^{4}\right)$ (cfr. (4.41)) we define the $3 \times 3$ complex Hermitian matrix

$$
J_{3}^{\mathbb{C}} \ni M=M^{\mathbb{C}}:=\left(\begin{array}{ccc}
x^{0}-x^{1} & -x^{2}-\mathbf{i} x^{3} & y^{1}+\mathbf{i} y^{3} \\
-x^{2}+\mathbf{i} x^{3} & x^{0}+x^{1} & y^{2}-\mathbf{i} y^{4} \\
y^{1}-\mathbf{i} y^{3} & y^{2}+\mathbf{i} y^{4} & s
\end{array}\right) .
$$

Notice that, consistently, this matrix is the restriction of $\mathcal{M}^{\mathbb{H}}$ (8.44) to $x^{4}=x^{5}=0$ and $y^{5}=\ldots=y^{8}=0$. Then we find

$$
\mathcal{V}_{L(2,1)}=-\operatorname{det} M
$$

\subsection{7 $L(1,1) \equiv J_{3}^{\mathbb{R}}$}

This model corresponds to a symmetric space [53], and it is related to the simple cubic Jordan algebra over the reals, $J_{3}^{\mathbb{R}}$, namely the algebra of symmetric real $3 \times 3$ matrices. In this case, the Bekenstein-Hawking entropy and the attractor values of scalar fields of extremal black holes are explicitly known; see e.g. [62]. In the present treatment, we will highlight its relation to the complete 'parent' models $L(8,1), L(4,1)$ and $L(2,1)$.

From (4.26), it holds that $\mathcal{D}_{2}=2$, and there are $1+3+2=6$ variables, namely $s, x^{0}, x^{1}, x^{2}, y^{1}, y^{2}$. The symmetric $2 \times 2 \Gamma$-matrices are $\Gamma_{0}=\mathbb{I}_{2}, \Gamma_{1}=\sigma_{3}, \Gamma_{2}=\sigma_{1}$, and thus $\left\{\Gamma_{1}, \Gamma_{2}\right\}$ is a Clifford set. The corresponding quadratic forms $Q_{I}={ }^{T} y \Gamma_{I} y$ from (5.28) read

$$
Q_{0} \equiv Q\left[\begin{array}{l}
0 \\
0
\end{array}\right], \quad Q_{1} \equiv Q\left[\begin{array}{l}
0 \\
1
\end{array}\right], \quad Q_{2} \equiv Q\left[\begin{array}{l}
1 \\
0
\end{array}\right]
$$

and again they can be obtained by suitably restricting the $y$-variables from the quadratic forms of $L(8,1)$ (or, equivalently, from $L(4,1)$ or from $L(2,1)$ ):

$$
\begin{aligned}
& \left(Q_{0}\right)_{\mid y^{3}=\ldots=y^{16}=0} \equiv Q\left[\begin{array}{l}
0 \\
0
\end{array}\right]:=\left(y^{1}\right)^{2}+\left(y^{2}\right)^{2} \\
& \left(Q_{1}\right)_{\mid y^{3}=\ldots=y^{16}=0} \equiv Q\left[\begin{array}{l}
0 \\
1
\end{array}\right]:=\left(y^{1}\right)^{2}-\left(y^{2}\right)^{2} ; \\
& \left(Q_{2}\right)_{\mid y^{3}=\ldots=y^{16}=0}=Q\left[\begin{array}{l}
1 \\
0
\end{array}\right]:=2 y^{1} y^{2} ; \\
& \left(Q_{3}\right)_{\mid y^{3}=\ldots=y^{16}=0}=0,
\end{aligned}
$$


thus implying

$$
\begin{aligned}
L(1,1) & =\left.L(8,1)\right|_{x^{3}=\ldots=x^{9}=0, y^{3}=\ldots=y^{16}=0} \\
& =\left.L(4,1)\right|_{x^{3}=x^{4}=x^{5}=0, y^{3}=\ldots=y^{8}=0} \\
& =\left.L(2,1)\right|_{x^{3}=0, y^{3}=y^{4}=0} ; \\
\mathcal{V}_{L(1,1)} & =\left.\mathcal{V}_{L(8,1)}\right|_{x^{3}=\ldots=x^{9}=0, y^{3}=\ldots=y^{16}=0} \\
& =\left.\mathcal{V}_{L(4,1)}\right|_{x^{3}=x^{4}=x^{5}=0, y^{3}=\ldots=y^{8}=0}=\left.\mathcal{V}_{L(2,1)}\right|_{x^{3}=0, y^{3}=y^{4}=0} .
\end{aligned}
$$

The following Lorentzian quadratic identity holds:

$$
-Q_{0}^{2}+Q_{1}^{2}+Q_{2}^{2}=0, \quad \text { in fact }\left(\left(y^{1}\right)^{2}+\left(y^{2}\right)^{2}\right)^{2}=\left(\left(y^{1}\right)^{2}-\left(y^{2}\right)^{2}\right)^{2}+\left(2 y^{1} y^{2}\right)^{2}
$$

The cubic form $\mathcal{V}$ on $\mathbb{R}^{6}$ is thus given by:

$$
\mathcal{V}_{L(1,1)}=s q(x)+\sum_{I=0}^{2} x^{I} Q_{I}(y),
$$

where the $x^{3}=x^{4}=x^{5}=x^{6}=x^{7}=x^{8}=x^{9}=0$ restriction of the Lorentzian quadratic form $q(x)$ (8.4) is the corresponding Lorentzian form in $x^{0}, x^{1}, x^{2}$ :

$$
q(x):=-\left(x^{0}\right)^{2}+q_{2}(x)=-\left(x^{0}\right)^{2}+\left(x^{1}\right)^{2}+\left(x^{2}\right)^{2} .
$$

(8.73) implies that the invertibility condition enounced in section 6.2 is satisfied with $r=0$, so $L(1,1)$ is a complete model. The inverse of the gradient map

$$
\nabla \mathcal{V}_{L(1,1)}: \mathbb{R}_{s, x, y}^{6} \longrightarrow \mathbb{R}_{t, u, v}^{6},
$$

is then simply the map $\mu$ in (6.17), whose components are homogeneous polynomials of degree 2:

$$
\begin{aligned}
& \mu: \mathbb{R}_{t, u, v}^{6} \equiv \mathbb{R}^{6} \longrightarrow \mathbb{R}_{s, x, y}^{6} \equiv \mathbb{R}^{6} ; \\
& \mu(t, u, v)=\left(\begin{array}{c}
q(u) \\
-2 t u_{0}+\frac{1}{2} Q_{0}(v) \\
2 t u_{1}+\frac{1}{2} Q_{1}(v) \\
2 t u_{2}+\frac{1}{2} Q_{2}(v) \\
\left(\sum_{I=0}^{2} u_{I} \Gamma_{I}\right) v
\end{array}\right)=\left(\begin{array}{c}
-u_{0}^{2}+u_{1}^{2}+u_{2}^{2} \\
-2 t u_{0}+\frac{1}{2}\left(v_{1}^{2}+v_{2}^{2}\right) \\
2 t u_{1}+\frac{1}{2}\left(v_{1}^{2}-v_{2}^{2}\right) \\
2 t u_{2}+v_{1} v_{2} \\
u_{0} v_{1}+u_{1} v_{1}+u_{2} v_{2} \\
u_{0} v_{2}-u_{1} v_{2}+u_{2} v_{1}
\end{array}\right) .
\end{aligned}
$$

The following identity, for any $\xi={ }^{T}(s, x, y) \in \mathbb{R}^{6}$ (cfr. (4.41)), holds:

$$
\mu\left(\nabla_{\mathcal{V}_{L(1,1)}}(\xi)\right)=4 \mathcal{V}_{L(1,1)}(\xi) \xi
$$


The determinant of $J_{3}^{\mathbb{R}}$, and an alternative construction of the map $\mu$. An alternative construction of the inverse map $\mu$ can be given, by exploiting the relation to $J_{3}^{\mathbb{R}}$. In fact, using the restriction of the matrix $M^{\mathbb{C}}(8.67)$ to $x^{3}=0$ and $y^{3}=y^{4}=0$, we get

$$
\mathcal{V}_{L(1,1)}=-\operatorname{det} \mathfrak{M}, \quad \mathfrak{M}:=\left(\begin{array}{ccc}
x^{0}-x^{1} & -x^{2} & y^{1} \\
-x^{2} & x^{0}+x^{1} & y^{2} \\
y^{1} & y^{2} & s
\end{array}\right) \in J_{3}^{\mathbb{R}} .
$$

Thus, the derivatives of $\mathcal{V}_{L(1,1)}$ are linear combinations of the $(2 \times 2)$-minors of $\mathfrak{M}$. By denoting with $M_{a b}$ the determinant of the submatrix of $\mathfrak{M}$ obtained by deleting the $a$-th row and $b$-th column, one obtains, with $\left(t, u^{0}, u^{1}, u^{2}, v^{1}, v^{2}\right)^{T}=\nabla_{\mathcal{V}_{L(1,1)}}$ :

$$
\begin{array}{ll}
t=\partial_{s} \mathcal{V}_{L(1,1)}=-M_{33}, & u_{2}=\partial_{x^{2}} \mathcal{V}_{L(1,1)}=-2 M_{12}, \\
u_{0}=\partial_{x^{0}} \mathcal{V}_{L(1,1)}=-M_{11}-M_{22}, & v_{1}=\partial_{y^{1}} \mathcal{V}_{L(1,1)}=-2 M_{13}, \\
u_{1}=\partial_{x^{1}} \mathcal{V}_{L(1,1)}=M_{11}-M_{22}, & v_{2}=\partial_{y^{2}} \mathcal{V}_{L(1,1)}=2 M_{23} .
\end{array}
$$

Therefore, $\nabla_{\mathcal{V}_{L(1,1)}}$ determines the coefficients of the adjoint matrix $\mathfrak{M}^{\sharp}$ :

$$
2 \mathfrak{M}^{\sharp}:=2\left(\begin{array}{ccc}
M_{11} & -M_{12} & M_{13} \\
-M_{12} & M_{22} & -M_{23} \\
M_{13} & -M_{23} & M_{33}
\end{array}\right)=\left(\begin{array}{ccc}
-u_{0}+u_{1} & u_{2} & -v_{1} \\
u_{2} & -u_{0}-u_{1} & -v_{2} \\
-v_{1} & -v_{2} & -2 t
\end{array}\right) .
$$

The inverse of $\nabla \mathcal{V}_{L(1,1)}$ is then basically given by ${ }^{26}$

$$
\mathfrak{M}^{\sharp} \mapsto\left(\mathfrak{M}^{\sharp}\right)^{\sharp}=(\operatorname{det} \mathfrak{M}) \mathfrak{M}=-\mathcal{V}_{L(1,1)}(\xi) \mathfrak{M},
$$

since $\mathfrak{M}$ determines $\xi$. Explicitly, one finds

$$
\left(2 \mathfrak{M}^{\sharp}\right)^{\sharp}=\left(\begin{array}{ccc}
2 t u_{0}+2 t u_{1}-v_{2}^{2} & 2 t u_{2}+v_{1} v_{2} & -u_{0} v_{1}-u_{1} v_{1}+u_{2} v_{2} \\
2 t u_{2}+v_{1} v_{2} & 2 t u_{0}-2 t u_{1}-v_{1}^{2} & -u_{0} v_{2}+u_{1} v_{2}-u_{2} v_{1} \\
-u_{0} v_{1}-u_{1} v_{1}-u_{2} v_{2} & -u_{0} v_{2}+u_{1} v_{2}-u_{2} v_{1} & u_{0}^{2}-u_{1}^{2}-u_{2}^{2}
\end{array}\right) .
$$

Next, since $\left(2 \mathfrak{M}^{\sharp}\right)^{\sharp}=4\left(\mathfrak{M}^{\sharp}\right)^{\sharp}=-4 \mathcal{V}_{L(1,1)}(\xi) \mathfrak{M}$ one recovers the inverse $\mu$ of $\nabla_{\mathcal{V}_{L(1,1)}}$ given in (8.77).

\section{$9 \quad$ Examples, II: $L(q, 2), q=1,2,3$}

In section 8.2 we showed that

$$
L(q, 1) \subset L(8,1), 1 \leqslant q \leqslant 7,
$$

with the 'inclusion' realized mostly by simply setting the last $x^{I}$-variables equal to zero, as well as by (occasionally) setting the 'last half' of the $y^{\alpha}$-variables to zero. In the present section, we will consider the inversion of the gradient map $\nabla_{\mathcal{V}}$ in models $L(q, 2)$, with $q=1,2,3$; in these models, it holds that (cfr. table 1 of [53])

$$
m \equiv \mathcal{D}_{q+1} \cdot 2=2^{q} \cdot 2=2^{q+1} .
$$

\footnotetext{
${ }^{26}$ This also results from the adjoint identity $(4.1)$ for $J_{3}^{\mathbb{R}}$, and as such this method actually works for all four complete models $L(q, 1)$ with $q=1,2,4,8$.
} 


\subsection{Block decompositions}

We define the $P$-block diagonal form $M^{(P)}$ of a matrix $M$ of size $m \times m$ as the matrix of size $m P \times m P$ defined as

$$
M^{(P)}:=M \otimes \mathbb{I}_{P}=\left(\begin{array}{ccc}
M & & \\
\vdots & \ddots & \vdots \\
& & M
\end{array}\right)
$$

For a vector $y \in \mathbb{R}^{m P}$, we define vectors $y^{(k)} \in \mathbb{R}^{m}$ by taking the vector with components $(k-1) P+1, \ldots, k P$ so that

$$
y={ }^{T}\left(y^{(1)}, \ldots, y^{(P)}\right) \in(\underbrace{\mathbb{R}^{m} \oplus \ldots \oplus \mathbb{R}^{m}}_{P \text { times }}=\mathbb{R}^{m P}) .
$$

Let now $\Gamma_{0}=\mathbb{I}_{m}$ and let $\left\{\Gamma_{1}, \ldots, \Gamma_{q+1}\right\}$ be a Clifford set of $m \times m$ matrices. The symmetric matrices $\Gamma_{I}^{(P)}$ are, in general, not $\Gamma$-matrices, since $m P$ is not a power of 2 . They still define quadratic forms on $\mathbb{R}^{m P}$, which we denote by $Q_{I}^{(P)}$ :

$$
Q_{I}^{(P)}(y):=Q_{I}\left(y^{(1)}\right)+Q_{I}\left(y^{(2)}\right)+\ldots+Q_{I}\left(y^{(P)}\right) .
$$

\section{$9.2 P=2$}

Given $\Gamma_{0}=\mathbb{I}_{m}$ and a Clifford set of $m \times m$ matrices $\left\{\Gamma_{1}, \ldots, \Gamma_{q+1}\right\}$, the matrices $\Gamma_{I}^{(2) \text {, s are }}$ again $\Gamma$-matrices of size $2 m=2^{g+1}$, with $\Gamma_{0}^{(2)}=\mathbb{I}_{2 m}$. Moreover, the set $\left\{\Gamma_{1}^{(2)}, \ldots, \Gamma_{q+1}^{(2)}\right\}$ is again a Clifford set, now of $2 m \times 2 m$ matrices, since distinct matrices still anti-commute and they square to $\mathbb{I}_{2 m}$. In the subsequent treatment, we will show that

$$
L(q, 2) \subset L\left(2^{q}, 1\right), q=1,2,3, \quad \text { that is: }\left\{\begin{array}{l}
L(1,2) \subset L(2,1), \\
L(2,2) \subset L(4,1), \\
L(3,2) \subset L(8,1),
\end{array}\right.
$$

where $\subset$ denotes a descendant relation. In each case, the quadratic forms $Q_{I}^{(2)}, I=$ $0, \ldots, q+1$, are all in the set of the $q^{\prime}+2$ quadratic forms defining the $L\left(q^{\prime}, 1\right)$ model. The remaining $r=q^{\prime}-q$ forms of the $L\left(q^{\prime}, 1\right)$ model (where $r=1,2,5$ respectively) can be used as the $R_{K}$ 's in the invertibility condition of section 6.2. In particular, the Lorentzian relation between the quadratic forms of the complete model implies the following Lorentzian quadratic relation (6.8) among the $Q_{I}^{(2)}$ 's and the $R_{K}$ 's:

$$
-Q_{0}^{(2)}(y)^{2}+Q_{1}^{(2)}(y)^{2}+\ldots+Q_{q+1}^{(2)}(y)^{2}+R_{1}(y)^{2}+\ldots+R_{r}(y)^{2}=0 .
$$

Thus, the cubic form $\mathcal{V}=\mathcal{V}_{L(q, 2)}$ for $q=1,2,3$ has an invertible gradient map $\nabla_{\mathcal{V}_{L(q, 2)}}$, and correspondingly the BPS system (2.6) can be explicitly solved.

Again, from the treatment of section 6, the solution of the BPS system of $L(q, 2)$ for $q=1,2,3$ is then given by (6.54)-(6.56), and (6.66) and (6.73)-(6.75) yield the corresponding expression of the BPS black hole entropy and of the BPS attractors, respectively. We refer to section 9.5, 10.6 for examples of lower dimensional models $L(q, P)$ (namely, $L(1,2)$ and $L(1,3)$ ) which we explicitly work out in detail (see also the generalization to $L(1, P)$ models with $P \geqslant 2$ in section 10.7$)$. 


\section{$9.3 L(3,2)$}

The $L(3,2)$ model has

$$
\left(1+q+2+P \cdot \mathcal{D}_{q+1}\right)_{q=3, P=2}=1+5+2 \cdot 8=22
$$

variables, denoted as $s, x^{0}, \ldots, x^{4}, y^{1}, \ldots, y^{16}$. The cubic form reads

$$
\mathcal{V}_{L(3,2)}=s q(x)+\sum_{I=0}^{4} x^{I} Q_{I}^{(2)}, \quad q(x):=-\left(x^{0}\right)^{2}+\left(x^{1}\right)^{2}+\left(x^{2}\right)^{2}+\left(x^{3}\right)^{2}+\left(x^{4}\right)^{2}
$$

where the $Q_{I}^{(2)}$ 's are given by (9.11) below.

Let us now show that $L(3,2) \subset L(8,1)$ : first of all, both models are defined by $\Gamma$ matrices of size $16 \times 16$, and let $\Gamma_{0}, \ldots, \Gamma_{9}$ be those of $L(8,1)$ as in section 8.1. We can write the five $\Gamma$-matrices (of size $8 \times 8$ ) of the $L(3,1)$ model as $\bar{\Gamma}_{0}=\mathbb{I}_{8}$ and the others $\left\{\bar{\Gamma}_{1}, \ldots, \bar{\Gamma}_{4}\right\}$ are a Clifford set. Obviously, $\Gamma_{0}=\bar{\Gamma}_{0}^{(2)}$. Next, we observe that $\Gamma_{1}, \Gamma_{3}, \Gamma_{4}, \Gamma_{5}$ (but not $\Gamma_{2}$ ) are of the form $\Gamma_{I}=\bar{\Gamma}_{J}^{(2)}$, for some $\Gamma$-matrices $\bar{\Gamma}_{J}$ of size $8 \times 8$. Moreover, these four $\bar{\Gamma}_{J}$ form a Clifford set (since the four $\Gamma_{I}$ are a Clifford set). Thus, we see that $L(3,2) \subset L(8,1)$, by using

$$
\bar{\Gamma}_{0}^{(2)}:=\Gamma_{0}, \quad \bar{\Gamma}_{1}^{(2)}:=\Gamma_{1}, \quad \bar{\Gamma}_{2}^{(2)}:=\Gamma_{3}, \quad \bar{\Gamma}_{3}^{(2)}:=\Gamma_{4}, \quad \bar{\Gamma}_{4}^{(2)}:=\Gamma_{5} .
$$

Below, we list the ten quadratic forms of the $L(8,1)$ model in the same order as in section 8.1 but with the names adapted to the $L(3,2)$ model. Five of the forms are denoted by $Q_{I}^{(2)}, I=0, \ldots, 4$, and these are in the $L(3,2)$ model, the remaining five, $R_{1}, \ldots, R_{5}$, will be used to satisfy the invertibility condition $^{27}$ enounced in section 6.2 (with $r=5$ ); cfr. (8.2):

$$
\begin{aligned}
Q_{0}^{(2)} \equiv Q\left[\begin{array}{l}
0000 \\
0000
\end{array}\right]:=\left(y^{1}\right)^{2}+\ldots+\left(y^{8}\right)^{2}+\left(y^{9}\right)^{2}+\ldots+\left(y^{16}\right)^{2} ; \\
Q_{1}^{(2)} \equiv Q\left[\begin{array}{l}
0000 \\
0001
\end{array}\right]:=\left\{\begin{array}{l}
\left(y^{1}\right)^{2}-\left(y^{2}\right)^{2}+\ldots+\left(y^{7}\right)^{2}-\left(y^{8}\right)^{2} \\
+\left(y^{9}\right)^{2}-\left(y^{10}\right)^{2}+\ldots+\left(y^{15}\right)^{2}-\left(y^{16}\right)^{2} ;
\end{array}\right. \\
R_{1} \equiv Q\left[\begin{array}{l}
0001 \\
1010
\end{array}\right]:=2\left(y^{1} y^{2}-y^{3} y^{4}+y^{5} y^{6}-y^{7} y^{8}-y^{9} y^{10}+y^{11} y^{12}-y^{13} y^{14}+y^{15} y^{16}\right) ; \\
Q_{2}^{(2)} \equiv Q\left[\begin{array}{l}
0011 \\
0000
\end{array}\right]:=2\left(y^{1} y^{4}+y^{2} y^{3}+y^{5} y^{8}+y^{6} y^{7}+y^{9} y^{12}+y^{10} y^{11}+y^{13} y^{16}+y^{14} y^{15}\right) ; \\
Q_{3}^{(2)} \equiv Q\left[\begin{array}{l}
0101 \\
0101
\end{array}\right]:=2\left(y^{1} y^{6}-y^{2} y^{5}+y^{3} y^{8}-y^{4} y^{7}+y^{9} y^{14}-y^{10} y^{13}+y^{11} y^{16}-y^{12} y^{15}\right) ; \\
Q_{4}^{(2)} \equiv Q\left[\begin{array}{l}
0111 \\
0110
\end{array}\right]:=2\left(y^{1} y^{8}+y^{2} y^{7}-y^{3} y^{6}-y^{4} y^{5}+y^{6} y^{16}+y^{10} y^{15}-y^{11} y^{14}-y^{12} y^{13}\right) ; \\
R_{2} \equiv Q\left[\begin{array}{l}
1001 \\
0010
\end{array}\right]:=2\left(y^{1} y^{10}+y^{2} y^{9}-y^{3} y^{12}-y^{4} y^{11}+y^{5} y^{14}+y^{6} y^{13}-y^{7} y^{16}-y^{8} y^{15}\right) ; \\
R_{3} \equiv Q\left[\begin{array}{l}
1011 \\
1101
\end{array}\right]:=2\left(y^{1} y^{12}-y^{2} y^{11}+y^{3} y^{10}-y^{4} y^{9}-y^{5} y^{16}+y^{6} y^{15}-y^{7} y^{14}+y^{8} y^{13}\right) ; \\
R_{4} \equiv Q\left[\begin{array}{l}
1101 \\
1110
\end{array}\right]:=2\left(y^{1} y^{14}+y^{2} y^{13}-y^{3} y^{16}-y^{4} y^{15}-y^{5} y^{10}-y^{6} y^{9}+y^{7} y^{12}+y^{8} y^{11}\right) ; \\
R_{5} \equiv Q\left[\begin{array}{l}
1111 \\
1001
\end{array}\right]:=2\left(y^{1} y^{16}-y^{2} y^{15}+y^{3} y^{14}-y^{4} y^{13}+y^{5} y^{12}-y^{6} y^{11}+y^{7} y^{10}-y^{8} y^{9}\right) .
\end{aligned}
$$

\footnotetext{
${ }^{27}$ Notice that the $Q_{I}^{(2)}$ listed here are not the quadrics obtained from the $Q_{I}$ 's in section 8.2 .5 , since we have chosen a different Clifford set for $L(3,1)$ in that section.
} 
Thus, (notice the peculiar choice of the variables, we put $x^{2}=0$ but $x^{5}$ remains) it holds that

$$
\begin{aligned}
& L(3,2)=\left.L(8,1)\right|_{x^{2}=x^{6}=x^{7}=x^{8}=x^{9}=0} \\
& \mathcal{V}_{L(3,2)}=\left.\mathcal{V}_{L(8,1)}\right|_{x^{2}=x^{6}=x^{7}=x^{8}=x^{9}=0}
\end{aligned} .
$$

Thus, up to a relabeling of the $x^{I}$ 's, the model $L(3,2)$ can be regarded as the $L(8,1)$ model with five linear constraints.

It is then clear that $L(3,2)$ is an invertible model: one may take the

$$
\Omega_{1}=\Gamma_{2}, \quad \Omega_{2}=\Gamma_{6}, \quad \Omega_{3}=\Gamma_{7}, \quad \Omega_{4}=\Gamma_{8}, \quad \Omega_{5}=\Gamma_{9}, \quad \text { so } \quad r=5,
$$

and, since the set of $\Gamma$-matrices $\left\{\Gamma_{1}, \ldots, \Gamma_{9}\right\}$ of $L(8,1)$ is a Clifford set and the Lorentzian identity (8.5) holds, the invertibility condition enounced in section 6.2 is satisfied.

Since $r \neq 0$, the inverse of the gradient map $\nabla_{\mathcal{V}_{L(3,2)}}$ is given as a composition of two maps, $\alpha$ and $\mu$ :

$$
\mathbb{R}_{\xi}^{1+5+16} \stackrel{\nabla_{\mathcal{V}_{L(3,2)}}^{\longrightarrow}}{\longrightarrow} \mathbb{R}_{z}^{1+5+16} \stackrel{\alpha}{\longrightarrow} \mathbb{R}_{(t, u, v, w)}^{1+5+16+5} \stackrel{\mu}{\longrightarrow} \mathbb{R}_{\xi}^{1+5+16}
$$

The map $\alpha$, as in (6.14), is given by

$$
\alpha\left(z_{1}, \ldots, z_{22}\right):={ }^{T}\left(z_{1}^{2}, z_{1} z_{2}, \ldots, z_{1} z_{22}, R_{1}(z), \ldots, R_{5}(z)\right),
$$

where the quadratic forms $R_{K}$ 's $(K=1, \ldots, 5)$ depend only on the last $2 \cdot 8=16$ variables and they are obtained by substituting $y^{L}:=z_{L+6}, L=1, \ldots, 16$ in $Q_{2}, Q_{6}, Q_{7}, Q_{8}, Q_{9}$ given in (8.2), respectively:

$$
\begin{aligned}
R_{1}(z):= & Q_{2}\left(z_{6}, \ldots, z_{22}\right), \\
R_{2}(z):= & Q_{6}\left(z_{6}, \ldots, z_{22}\right), \\
& \vdots \vdots \\
R_{5}(z):= & Q_{9}\left(z_{6}, \ldots, z_{22}\right) .
\end{aligned}
$$

The map $\mu$ (6.17) has $1+5+16+5$ components which are homogeneous polynomials of degree 2 in the 27 variables $t, u_{0}, \ldots, u_{4}, v_{1}, \ldots, v_{16}, w_{1}, \ldots, w_{5}$. Since

$$
\mu \circ \alpha \circ \nabla \mathcal{V}_{L(3,2)}(\xi)=4 q(x)^{2} \mathcal{V}_{L(3,2)}(\xi) \xi,
$$

where $\mathcal{V}_{L(3,2)}$ and the corresponding $q(x)$ are given by (9.9), the (birational) inverse of the gradient map $\nabla_{\mathcal{V}_{L(3,2)}}$ is the map $\mu \circ \alpha$, which is an homogeneous polynomial map of degree four.

\section{$9.4 L(2,2)$}

The $L(2,2)$ model has $q=2, P=2$, and $\mathcal{D}_{3}=4$ [53]; thus, the number of variables is

$$
\left(1+q+2+P \cdot \mathcal{D}_{q+1}\right)_{q=2, P=2}=1+4+2 \cdot 4=13 .
$$


Correspondingly, the cubic form reads as follows:

$$
\mathcal{V}_{L(2,2)}=s q(x)+\sum_{I=0}^{3} x^{I} Q_{I}^{(2)}, \quad q(x):=-\left(x^{0}\right)^{2}+\left(x^{1}\right)^{2}+\left(x^{2}\right)^{2}+\left(x^{3}\right)^{2}
$$

where the $Q_{I}^{(2)}$ 's are given by (9.24) below. Within the black hole effective potential formalism, this model has been treated in [51].

Let us now show that $L(2,2) \subset L(4,1)$ : we write the four $\Gamma$-matrices (of size $4 \times 4$ ) of the $L(2,1)$ model given in section 8.2.6 as $\bar{\Gamma}_{0}=\mathbb{I}_{4}$, and the others $\left\{\bar{\Gamma}_{1}, \ldots, \bar{\Gamma}_{3}\right\}$ are a Clifford set. Next, we observe that if $\Gamma_{I}, I=0, \ldots, 5$ are the six $\Gamma$-matrices of the $L(4,1)$ model in 8.2.4, then we see that $L(2,2) \subset L(4,1)$, since

$$
\bar{\Gamma}_{0}^{(2)}:=\Gamma_{0}, \quad \bar{\Gamma}_{1}^{(2)}:=\Gamma_{1}, \quad \bar{\Gamma}_{2}^{(2)}:=\Gamma_{2}, \quad \bar{\Gamma}_{3}^{(2)}:=\Gamma_{3} .
$$

Below, we list the six quadratic forms of the $L(4,1)$ model in the same order as in section 8.2.4, but with the names adapted to the $L(3,2)$ model. Four of the forms are denoted by $Q_{I}^{(2)}, I=0, \ldots, 3$, and these are in the $L(2,2)$ model; the remaining two, $R_{1}, R_{2}$, will be used to satisfy the invertibility condition enounced in section 6.2 (with $r=2$ ). Notice that both models are defined by $\Gamma$-matrices of size $8 \times 8$; let $\Gamma_{0}, \ldots, \Gamma_{5}$ be those of $L(4,1)$ as in section 8.2.4.

$$
\begin{aligned}
& \bar{\Gamma}_{0}^{(2)}=\mathbb{I}_{8}, \quad Q_{0}^{(2)} \equiv Q\left[\begin{array}{l}
0000 \\
000
\end{array}\right]:=\left\{\begin{array}{l}
\left(y^{1}\right)^{2}+\left(y^{2}\right)^{2}+\left(y^{3}\right)^{2}+\left(y^{4}\right)^{2} \\
+\left(y^{5}\right)^{2}+\left(y^{6}\right)^{2}+\left(y^{7}\right)^{2}+\left(y^{8}\right)^{2}
\end{array}\right. \\
& \bar{\Gamma}_{1}^{(2)}=\sigma_{3} \otimes \mathbb{I}_{2} \otimes \mathbb{I}_{2}, \quad Q_{1}^{(2)} \equiv Q\left[\begin{array}{l}
000 \\
001
\end{array}\right]:=\left\{\begin{array}{l}
\left(y^{1}\right)^{2}-\left(y^{2}\right)^{2}+\left(y^{3}\right)^{2}-\left(y^{4}\right)^{2} \\
+\left(y^{5}\right)^{2}-\left(y^{6}\right)^{2}+\left(y^{7}\right)^{2}-\left(y^{8}\right)^{2} ;
\end{array}\right. \\
& \bar{\Gamma}_{2}^{(2)}=\sigma_{1} \otimes \sigma_{3} \otimes \mathbb{I}_{2}, \quad Q_{2}^{(2)} \equiv Q\left[\begin{array}{l}
001 \\
010
\end{array}\right]:=2\left(y^{1} y^{2}-y^{3} y^{4}+y^{5} y^{6}-y^{7} y^{8}\right) ; \\
& \bar{\Gamma}_{3}^{(2)}=\sigma_{1} \otimes \sigma_{1} \otimes \mathbb{I}_{2}, \quad Q_{3}^{(2)} \equiv Q\left[\begin{array}{l}
011 \\
000
\end{array}\right]:=2\left(y^{1} y^{4}+y^{2} y^{3}+y^{5} y^{8}+y^{6} y^{7}\right) ; \\
& \Omega_{1}=\gamma_{11} \otimes \mathbb{I}_{2} \otimes \gamma_{11}, R_{1} \equiv Q\left[\begin{array}{l}
101 \\
101
\end{array}\right]:=2\left(y^{1} y^{6}-y^{2} y^{5}+y^{3} y^{8}-y^{4} y^{7}\right) \text {; } \\
& \Omega_{2}=\sigma_{1} \otimes \gamma_{11} \otimes \gamma_{11}, R_{2} \equiv Q\left[\begin{array}{l}
111 \\
110
\end{array}\right]:=2\left(y^{1} y^{8}+y^{2} y^{7}-y^{3} y^{6}-y^{4} y^{5}\right) \text {. }
\end{aligned}
$$

Thus, it holds that

$$
\begin{aligned}
& L(2,2)=\left.L(4,1)\right|_{x^{4}=x^{5}=0} ; \\
& \mathcal{V}_{L(2,2)}=\left.\mathcal{V}_{L(4,1)}\right|_{x^{4}=x^{5}=0}
\end{aligned}
$$

Namely, the model $L(2,2)$ can be regarded as the $L(4,1)$ model with two linear constraints.

It is then clear that the model $L(2,2)$ is invertible. Indeed, in order to invert the gradient map $\nabla_{\mathcal{V}_{L(2,2)}}$, in the condition of section 6.2 , one can take

$$
\Omega_{1}:=\Gamma_{4}, \quad \Omega_{2}:=\Gamma_{5} \quad \text { so } \quad R_{1}(y)=Q_{4}(y), \quad R_{2}(y)=Q_{5}(y), \quad r=2 .
$$

Using that the set of $\Gamma$-matrices $\left\{\Gamma_{1}, \ldots, \Gamma_{5}\right\}$ of $L(4,1)$ is a Clifford set and that the Lorentzian identity (8.37), which now reads

$$
-Q_{0}^{(2)}(y)^{2}+Q_{1}^{(2)}(y)^{2}+Q_{2}^{(2)}(y)^{2}+Q_{3}^{(2)}(y)^{2}+R_{1}(y)^{2}+R_{2}(y)^{2}=0,
$$

holds, the condition of section 6.2 is satisfied. 
Since $r=2$, the inverse of the gradient map $\nabla_{\mathcal{V}_{L(2,2)}}$ is given as a composition of two maps, $\alpha$ and $\mu$ (cfr. (4.41)):

$$
\mathbb{R}_{\xi}^{1+4+8} \stackrel{\nabla \mathcal{V}_{L(2,2)}}{\longrightarrow} \mathbb{R}_{z}^{1+4+8} \stackrel{\alpha}{\longrightarrow} \mathbb{R}_{(t, u, v, w)}^{1+4+8+2} \stackrel{\mu}{\longrightarrow} \mathbb{R}_{\xi}^{1+4+8} .
$$

The map $\alpha$, as in (6.14), is given by

$$
\alpha\left(z_{1}, \ldots, z_{13}\right):={ }^{T}\left(z_{1}^{2}, z_{1} z_{2}, \ldots, z_{1} z_{13}, R_{1}(z), R_{2}(z)\right),
$$

where the quadratic forms $R_{K}$ 's $(K=1,2)$ depend only on the last $2 \cdot 4=8$ variables and they are obtained by substituting $y^{L}:=z_{L+5}, L=1, \ldots, 8$ in $\bar{Q}_{4}$ and $\bar{Q}_{5}$ given in (8.36), respectively:

$$
\begin{aligned}
& R_{1}(z)=2\left(z_{6} z_{11}-z_{7} z_{10}+z_{8} z_{13}-z_{9} z_{12}\right) \\
& R_{2}(z)=2\left(z_{6} z_{13}+z_{7} z_{12}-z_{8} z_{11}-z_{9} z_{10}\right)
\end{aligned}
$$

The map $\mu$ (6.17) has $1+4+8+2$ components that are homogeneous polynomials of degree 2 in the variables $t, u_{0}, \ldots, u_{3}, v_{1}, \ldots, v_{8}, w_{1}, w_{2}$. Since

$$
\mu \circ \alpha \circ \nabla_{\mathcal{V}_{L(2,2)}}(\xi)=4 q(x)^{2} \mathcal{V}_{L(2,2)}(\xi) \xi,
$$

where $\mathcal{V}_{L(2,2)}$ and the corresponding $q(x)$ are given by (9.22), the (birational) inverse of the gradient map $\nabla_{\mathcal{V}_{L(2,2)}}$ is the map $\mu \circ \alpha$, which is an homogeneous polynomial map of degree four.

\section{$9.5 \quad L(1,2)$}

The model $L(1,2)$ has $\mathcal{D}_{2}=2$ (cfr. (4.26)). The total dimension of the underlying vector space is then

$$
\left(1+q+2+P \cdot \mathcal{D}_{q+1}\right)_{q=1, P=2}=1+3+2 \cdot 2=8,
$$

and the corresponding variables split as $s, x^{0}, x^{1}, x^{2}, y^{1} \ldots, y^{4}$; thus, in this model $I=0,1,2$ and $\alpha=1, \ldots, 4$. Despite the treatment, within a different formalism, in [6] and in [51], to the best of our knowledge, the BPS black hole entropy and attractors for such a model were not previously known in literature in terms of the electric and magnetic black hole charges. It is here worth remarking that the $L(1,2)$ model is one of the simplest models related to an homogeneous non-symmetric 'special' manifold; therefore, in appendix A we work out the computations in full detail.

\section{Examples, III: $L(q, P), q=1,2,3, P \geqslant 3$}

In this section we consider the models $L(q, P)$ with $P \geqslant 3$ and $q=1,2,3$ : we will show that these models have an invertible gradient map. ${ }^{28}$ While for the models with $P=1,2$ considered above the embedding as a linear section into a complete model $L\left(q^{\prime}, 1\right)$ (with $q^{\prime}=2,4$, or 8 ) was crucial in order to establish invertibility, for the models with $P \geqslant 3$ treated below it suffices that the invertibility condition of section 6.2 is satisfied (for a fixed $q$ ) and $P=1$ and 2 ; the invertibility for all other $P \geqslant 3$ then comes "for free".

\footnotetext{
${ }^{28} \mathrm{As}$ to now, we don't know if this is the case also for other $q$ 's $\geqslant 4$; see section 15 .
} 


\subsection{Block $(k, l)$-lifts}

In order to show that the gradient map of a model $L(q, P)$, with $q=1,2,3$ and $P \geqslant 3$ is invertible by the condition enounced in section 6.2 , we need to find symmetric matrices $\Omega_{K}$ satisfying the anti-commutativity conditions (6.6) and the Lorentz identity (6.8).

To this aim, we will 'lift' the $\Omega_{K}$ 's, of size $2 m$, from the $P=2$ models discussed in section 9 to matrices $\Omega_{K}^{(k l)}$ of size $m P$ for any $P \geqslant 3$. For $y=\left(y^{(1)}, \ldots, y^{(P)}\right) \in \mathbb{R}^{m P}$ as in (9.4) and $k, l$ with $1 \leqslant k<l \leqslant P$, we define the following vector in $\mathbb{R}^{2 m}$ :

$$
y^{(k l)}:={ }^{T}\left(y^{(k)}, y^{(l)}\right) \quad \in \mathbb{R}^{2 m} .
$$

Next, for a quadratic form $R$ in $2 m$ variables, we define quadratic forms in $m P$ variables, with $P \geqslant 2$, which actually depend only on $2 m$ of them, namely on the $y$ 's belonging to the blocks $k$ and $l$ (notice that we suppress $P$ from the notation):

$$
R^{(k, l)}(y):=R\left(y^{(k l)}\right) \quad\left(y \in \mathbb{R}^{m P}\right) .
$$

Given the matrices $\Omega_{K}$ of size $m \times m$ of the $L(q, 2)$ model $(q=1,2,3)$ discussed in section 9 , let as before $R_{K}$ be the quadratic form in $2 m$ variables defined by $\Omega_{K}$ and let $\Omega_{K}^{(k l)}$ be the symmetric matrix of size $m P$ defined by the quadratic form $R_{K}^{(k, l)}$ in $m P$ variables, so that

$$
R_{K}^{(k, l)}(y)=R_{K}\left(y^{(k l)}\right)={ }^{T} y \Omega_{K}^{(k l)} y \quad\left(y \in \mathbb{R}^{m P}\right) .
$$

\subsection{Invertibility}

The $\Gamma$-matrices, of size $m P \times m P$, of the $L(q, P)(P \geqslant 3)$ models are the $q+2$ matrices $\Gamma_{I}^{(P)}:=\Gamma_{I} \otimes \mathbb{I}_{P}, I=0, \ldots, q+1$, where the $\Gamma_{I}$ are the $\Gamma$-matrices of the $L(q, 1)$ model.

Now, we claim that if we consider the $m P \times m P$ matrices $\Omega_{K}^{(k l)}, 1 \leqslant k<l \leqslant P$ where the $\Omega_{K}$ are the extra matrices in the $L(q, 2)$ model, then the conditions (6.6) and (6.8) for the invertibility of the gradient map of these $L(q, P)$ models $(P \geqslant 3)$ are all satisfied.

The anti-commutativity condition follows rather trivially from the fact that in section 9 we checked that the $\Gamma_{I}^{(2)}, I=1, \ldots, q+1$, anti-commute with all the $\Omega_{K}$ 's of the $L(q, 2)$ models we have considered, and therefore also the $\Gamma$-matrices $\Gamma_{I}^{(P)}$ of the $L(q, P)$ models (with $P \geqslant 3$ ) anti-commute with all the $\Omega_{K}^{(k l)}$, so (6.6) is satisfied.

On the other hand, in section 10.3 below, we will check the existence of the Lorentzian identity (6.8), namely we will verify the following Lorentzian identity between quadratic forms in $m P$ variables for all $P \geqslant 3$ :

$$
-Q_{0}^{(P)}(y)^{2}+Q_{1}^{(P)}(y)^{2}+\ldots+Q_{q+1}^{(P)}(y)^{2}+\sum_{K=1}^{r} \sum_{1 \leqslant k<l \leqslant P} R_{K}^{(k, l)}(y)^{2}=0 .
$$

Thus, the invertibility condition of section 6.2 is satisfied and the corresponding gradient map (and BPS system) can be inverted for any $q=1,2,3$ and $P \geqslant 3$.

Again, from the treatment of section 6, the solution of the BPS system of $L(q, P)$ for $q=1,2,3$ and $P \geqslant 3$ is then given by (6.54)-(6.56), and (6.66) and (6.73)-(6.75) yield 
the corresponding expression of the BPS black hole entropy and of the BPS attractors, respectively. We refer to section 9.5, 10.6 for examples of lower dimensional models $L(q, P)$ (namely, $L(1,2)$ and $L(1,3)$ ) which we explicitly work out in detail (see also the generalization to $L(1, P)$ models with $P \geqslant 2$ in section 10.7$)$.

\subsection{Proof of the Lorentzian identity (10.4)}

The definition (9.5) of $Q_{I}^{(P)}$ 's allows us to rewrite the first terms in the l.h.s. of (10.4) as follows:

$$
\begin{aligned}
& -Q_{0}^{(P)}(y)^{2}+\sum_{I=1}^{q+1} Q_{I}^{(P)}(y)^{2} \\
= & -\left(\sum_{k=1}^{P} Q_{0}\left(y^{(k)}\right)\right)^{2}+\sum_{I=1}^{q+1}\left(\sum_{k=1}^{P} Q_{I}\left(y^{(k)}\right)\right)^{2} \\
= & \sum_{k=1}^{P}\left(-Q_{0}\left(y^{(k)}\right)^{2}+\sum_{I=1}^{q+1} Q_{I}\left(y^{(k)}\right)^{2}\right)-\sum_{1 \leqslant k<l \leqslant P} S_{0, k, l}+\sum_{I=1}^{q+1} \sum_{1 \leqslant k<l \leqslant P} S_{I, k, l},
\end{aligned}
$$

where the remaining cross terms are defined as follows (no sum on repeated indices, $I=0,1, \ldots, q+1)$ :

$$
S_{I, k, l}:=2 Q_{I}\left(y^{(k)}\right) Q_{I}\left(y^{(l)}\right) .
$$

- We do the cases $q=1,2$ first. The Lorentzian quadratic relations (8.73) resp. (8.61) hold, thus for any $k$ we find that

$$
-Q_{0}\left(y^{(k)}\right)^{2}+\sum_{I=1}^{q+1} Q_{I}\left(y^{(k)}\right)^{2}=0
$$

which trivially implies

$$
\sum_{k=1}^{P}\left(-Q_{0}\left(y^{(k)}\right)^{2}+\sum_{I=1}^{q+1} Q_{I}\left(y^{(k)}\right)^{2}\right)=0 .
$$

Consequently, it remains to show that

$$
-\sum_{1 \leqslant k<l \leqslant P} S_{0, k, l}+\sum_{I=1}^{q+1} \sum_{1 \leqslant k<l \leqslant P} S_{I, k, l}+\sum_{K=1}^{r} \sum_{1 \leqslant k<l \leqslant P} R_{K}^{(k, l)}(y)^{2}=0 .
$$

In the Lorentzian identity (9.7) for the $L(q, 2)$ models, the vector $y$ is in fact $y^{(12)}$; so, replacing it by $y^{(k l)}$, one obtains the identities, for any $1 \leqslant k<l \leqslant P$ :

$$
-Q_{0}^{(2)}\left(y^{(k l)}\right)^{2}+Q_{1}^{(2)}\left(y^{(k l)}\right)^{2}+\ldots+Q_{q+1}^{(2)}\left(y^{(k l)}\right)^{2}+R_{1}\left(y^{(k l)}\right)^{2}+\ldots+R_{r}\left(y^{(k l)}\right)^{2}=0 .
$$

Recalling the definition (9.5) of the quadratic forms $Q_{I}^{(2)}$, one gets

$$
Q_{I}^{(2)}\left(y^{(k l)}\right)^{2}=\left(Q_{I}\left(y^{(k)}\right)+Q_{I}\left(y^{(l)}\right)\right)^{2}=Q_{I}\left(y^{(k)}\right)^{2}+Q_{I}\left(y^{(l)}\right)^{2}+2 Q_{I}\left(y^{(k)}\right) Q_{I}\left(y^{(l)}\right) .
$$


By plugging (10.11) into (10.10), two copies (one for $y^{(k)}$ and one for $y^{(l)}$ ) of the identity (10.7) are obtained, and there remains, for any pair $(k, l)$ with $1 \leqslant k<l \leqslant P$, the identity:

$$
\begin{aligned}
& 2\left(-Q_{0}\left(y^{(k)}\right) Q_{0}\left(y^{(l)}\right)+\sum_{I=1}^{q+1} Q_{I}\left(y^{(k)}\right) Q_{I}\left(y^{(l)}\right)\right) \\
& +R_{1}\left(y^{(k l)}\right)^{2}+\ldots+R_{r}\left(y^{(k l)}\right)^{2}=0 .
\end{aligned}
$$

By recalling the definition (10.6) of $S_{I, k, l}$, we see that (10.12) is the identity

$$
-S_{0, k, l}+\sum_{I=1}^{q+1} S_{I, k, l}+\sum_{K=1}^{r} R_{K}^{(k, l)}(y)^{2}=0 .
$$

Now we just sum over all $k, l$ and we obtain (10.9), which in turn implies the identity (10.4).

- Let us now consider the case $q=3$. In this case we do not have a Lorentzian relation (10.7) but the $L(3,1)$ model has an auxiliary quadratic form $R$ (cfr. section 8.2.5). We first take a closer look at the $L(3,2)$ model from section 9.3 , which was shown to be a descendant of $L(8,1)$. The main point of interest is the (auxiliary) quadratic form $R_{1}$ in $2 m=16$ variables $y^{1}, \ldots, y^{16}$, which is $Q_{2}$ in the $L(8,1)$ model, and which can be written as

$$
R_{1}(y)=R\left(y^{(1)}\right)-R\left(y^{(2)}\right), R(z)=2\left(z_{1} z_{2}-z_{3} z_{4}+z_{5} z_{6}-z_{7} z_{8}\right), y={ }^{T}\left(y^{(1)}, y^{(2)}\right) \in \mathbb{R}^{16}
$$

Next, we characterize the $L(3,1)$ model not as done in section 8.2 .5 , but rather as a linearly constrained $L(3,2)$ model:

$$
L(3,1)=L(3,2)_{y^{9}=\ldots=y^{16}=0} .
$$

Within this approach, the five quadrics $Q_{0}, \ldots, Q_{4}$ from $L(3,1)$ are the restrictions of the quadrics of the same name from $L(3,2)$, which again are $Q_{0}, Q_{1}, Q_{3}, Q_{4}, Q_{5}$ respectively of the $L(8,1)$ model, and the auxiliary quadric of $L(3,1)$ is the restriction of $^{29} Q_{2}$. With these conventions, we have the Lorentzian relation

$$
-Q_{0}(y)^{2}+\sum_{I=1}^{4} Q_{I}(y)^{2}+R(y)^{2}=0
$$

holding for the $L(3,1)$ model, as well as the Lorentzian relation for the $L(3,2)$ model:

$$
-Q_{0}^{(2)}\left(y^{(12)}\right)^{2}+\sum_{I=1}^{4} Q_{I}^{(2)}\left(y^{(12)}\right)^{2}+R_{1}\left(y^{(12)}\right)+R_{2}\left(y^{(12)}\right)+\ldots+R_{5}\left(y^{(12)}\right)=0 .
$$

From (10.14), we obtain

$$
R_{1}^{(k, l)}(y)^{2}=R_{1}\left(y^{(k l)}\right)^{2}=R\left(y^{(k)}\right)^{2}+R\left(y^{(l)}\right)^{2}-S_{R, k, l}, \quad S_{R, k, l}:=2 R\left(y^{(k)}\right) R\left(y^{(l)}\right) .
$$

\footnotetext{
${ }^{29}$ Note that in the treatment of the $L(3,1)$ model in section 8.2.5 we have interchanged the roles of $Q_{2}$ and $Q_{5}$ from $L(8,1)$.
} 
Using this, (10.11) and (10.16), we obtain from (10.17), for any pair $(k, l)$ with $1 \leqslant k<l \leqslant P$ the identity:

$$
\begin{aligned}
& 2\left(-Q_{0}\left(y^{(k)}\right) Q_{0}\left(y^{(l)}\right)+\sum_{I=1}^{4} Q_{I}\left(y^{(k)}\right) Q_{I}\left(y^{(l)}\right)-R\left(y^{(k)}\right) R\left(y^{(l)}\right)\right) \\
& \quad+R_{2}\left(y^{(k l)}\right)^{2}+\ldots+R_{5}\left(y^{(k l)}\right)^{2}=0
\end{aligned}
$$

which can be rewritten as

$$
-S_{0, k, l}+\sum_{I=1}^{4} S_{I, k, l}-S_{R, k, l}+\sum_{K=2}^{5} R_{K}^{(k, l)}(y)^{2}=0 .
$$

Finally, using (10.5) and (10.16), we obtain

$$
-Q_{0}^{(P)}(y)^{2}+\sum_{I=1}^{4} Q_{I}^{(P)}(y)^{2}+\sum_{k<l} R_{1}^{(k, l)}(y)^{2}=\sum_{1 \leqslant k<l \leqslant P}\left(-S_{0, k, l}+\sum_{I=1}^{4} S_{I, k, l}-S_{R, k, l}\right) .
$$

Combining (10.20), summed over $k, l$, and (10.21) we conclude that (10.4) holds also for $q=3$.

\section{$10.4 L(3,3)$}

The $L(3,3)$ model has $q=3, P=3$, and $\mathcal{D}_{3}=8$ [53]; thus, the number of variables is

$$
\left(1+q+2+P \cdot \mathcal{D}_{q+1}\right)_{q=3, P=3}=1+5+24=30 .
$$

The $\Gamma^{(3)}$-matrices, having size $24 \times 24$, are not $\Gamma$-matrices (differently from the $\Gamma^{(2)}$-matrices). This model has five $\Gamma$-matrices $\Gamma_{I}^{(3)}(I=0, \ldots, 4)$, obtained from the five $\Gamma$-matrices of the $L(3,1)$ model and defining the quadratic forms $Q_{I}^{(3)}$ as in $(9.5)$ :

$$
Q_{I}^{(3)}\left(y^{1}, \ldots, y^{24}\right)=Q_{I}\left(y^{1}, \ldots, y^{8}\right)+Q_{I}\left(y^{9}, \ldots, y^{16}\right)+Q_{I}\left(y^{17}, \ldots, y^{24}\right) .
$$

For obvious reasons, we refrain from writing them down explicitly. Next, there are the are $3 \cdot 5=15$ matrices $\Omega_{K}^{(k l)}$ with $K=1, \ldots, 5$ and $1 \leqslant k<l \leqslant 3$ which define the quadratic forms $R_{K}^{(k, l)}$. Starting from the five quadratic forms $R_{K}$ in 16 variables from the $L(3,2)$ model (given in (9.11)), one finds these quadratic forms $R_{K}^{(k, l)}$ in 24 variables $y^{\alpha}$ (but actually each on depends only on 16 of these variables) as in (10.2):

$$
R_{K}^{(k, l)}(y)=R_{K}\left(y^{(k l)}\right),
$$

so, for $K=1, \ldots, 5$ :

$$
\begin{aligned}
& R_{K}^{(1,2)}\left(y^{1}, \ldots, y^{24}\right)=R_{K}\left(y^{1}, \ldots, y^{8}, y^{9}, \ldots, y^{16}\right) \\
& R_{K}^{(1,3)}\left(y^{1}, \ldots, y^{24}\right)=R_{K}\left(y^{1}, \ldots, y^{8}, y^{17}, \ldots, y^{24}\right) \\
& R_{K}^{(2,3)}\left(y^{1}, \ldots, y^{24}\right)=R_{K}\left(y^{9}, \ldots, y^{16}, y^{17}, \ldots, y^{24}\right) .
\end{aligned}
$$


The matrices (except for $\Gamma_{0}^{(3)}=\mathbb{I}_{24}$ ) anti-commute (cfr. section 10.2 for the general case) and it can be verified (see 10.3 for the general case) that they satisfy the Lorenzian quadratic relation given by (10.4), with $P=3$ :

$$
-Q_{0}^{(3)}(y)^{2}+Q_{1}^{(3)}(y)^{2}+\ldots+Q_{4}^{(3)}(y)^{2}+\sum_{K=1}^{5} \sum_{1 \leqslant k<l \leqslant 3} R_{K}^{(k, l)}(y)^{2}=0 .
$$

Therefore, the inverse of the gradient map $\nabla_{\mathcal{V}_{L(3,3)}}$ of the cubic form

$$
\mathcal{V}_{L(3,3)}:=s q(x)+\sum_{I=0}^{4} x^{I} Q_{I}^{(3)}(y), \text { with } \quad q(x)=-\left(x^{0}\right)^{2}+\left(x^{1}\right)^{2}+\ldots+\left(x^{4}\right)^{2}
$$

can then be found following the procedure discussed in section 6.4.

\section{$10.5 \quad L(2,3)$}

The $L(2,3)$ model has $q=2, P=3$, and $\mathcal{D}_{3}=4$ [53]; thus, the number of variables is

$$
\left(1+q+2+P \cdot \mathcal{D}_{q+1}\right)_{q=2, P=3}=1+4+12=17 .
$$

The $\Gamma^{(3)}$-matrices have size $12 \times 12$, thus, differently from the $\Gamma^{(2)}$-matrices, they are not $\Gamma$-matrices; besides the four $\Gamma_{I}^{(3)}$, s $(I=0,1,2,3)$, there are the $3+3$ matrices $\Omega_{K}^{(k l)}$ with $K=1,2$ and $1 \leqslant k<l \leqslant 3$, which define the quadratic forms $R_{K}^{(k, l)}$. The quadratic forms involved are

$$
\begin{aligned}
Q_{0}^{(3)} & =\left\{\begin{array}{l}
\left(y^{1}\right)^{2}+\left(y^{2}\right)^{2}+\left(y^{3}\right)^{2}+\left(y^{4}\right)^{2}+\left(y^{5}\right)^{2}+\left(y^{6}\right)^{2}+\left(y^{7}\right)^{2} \\
+\left(y^{8}\right)^{2}+\left(y^{9}\right)^{2}+\left(y^{10}\right)^{2}+\left(y^{11}\right)^{2}+\left(y^{12}\right)^{2}
\end{array}\right. \\
Q_{1}^{(3)} & =\left\{\begin{array}{l}
\left(y^{1}\right)^{2}-\left(y^{2}\right)^{2}+\left(y^{3}\right)^{2}-\left(y^{4}\right)^{2}+\left(y^{5}\right)^{2}-\left(y^{6}\right)^{2}+\left(y^{7}\right)^{2} \\
-\left(y^{8}\right)^{2}+\left(y^{9}\right)^{2}-\left(y^{10}\right)^{2}+\left(y^{11}\right)^{2}-\left(y^{12}\right)^{2}
\end{array}\right. \\
Q_{2}^{(3)} & =2\left(y^{1} y^{2}-y^{3} y^{4}+y^{5} y^{6}-y^{7} y^{8}+y^{9} y^{10}-y^{11} y^{12}\right) \\
Q_{3}^{(3)} & =2\left(y^{1} y^{4}+y^{2} y^{3}+y^{5} y^{8}+y^{6} y^{7}+y^{9} y^{12}+y^{10} y^{11}\right) \\
R_{1}^{(1,2)} & =2\left(y^{1} y^{6}-y^{2} y^{5}+y^{3} y^{8}-y^{4} y^{7}\right) \\
R_{1}^{(1,3)} & =2\left(y^{1} y^{10}-y^{2} y^{9}+y^{3} y^{12}-y^{4} y^{11}\right) \\
R_{1}^{(2,3)} & =2\left(y^{5} y^{10}-y^{6} y^{9}+y^{7} y^{12}-y^{8} y^{11}\right) \\
R_{2}^{(1,2)} & =2\left(y^{1} y^{8}+y^{2} y^{7}-y^{3} y^{6}-y^{4} y^{5}\right) \\
R_{2}^{(1,3)} & =2\left(y^{1} y^{12}+y^{2} y^{11}-y^{3} y^{10}+y^{4} y^{9}\right) \\
R_{2}^{(2,3)} & =2\left(y^{5} y^{12}+y^{6} y^{11}-y^{7} y^{10}+y^{8} y^{9}\right)
\end{aligned}
$$

The matrices (except for $\Gamma_{0}^{(3)}=\mathbb{I}_{12}$ ) anti-commute (cfr. section 10.2 for the general case) and it can be verified (see 10.3 for the general case) that they satisfy the Lorenzian quadratic relation given by (10.4), with $P=3$ :

$$
-Q_{0}^{(3)}(y)^{2}+Q_{1}^{(3)}(y)^{2}+Q_{2}^{(3)}(y)^{2}+Q_{3}^{(3)}(y)^{2}+\sum_{K=1}^{2} \sum_{1 \leqslant k<l \leqslant 3} R_{K}^{(k, l)}(y)^{2}=0 .
$$


Therefore, the inverse of the gradient map $\nabla_{\mathcal{V}_{L(2,3)}}$ of the cubic form

$$
\mathcal{V}_{L(2,3)}:=s q(x)+\sum_{I=0}^{3} x^{I} Q_{I}^{(3)}(y), \text { with } \quad q(x)=-\left(x^{0}\right)^{2}+\left(x^{1}\right)^{2}+\left(x^{2}\right)^{2}+\left(x^{3}\right)^{2}
$$

can then be found following the procedure discussed in section 6.4.

\section{$10.6 L(1,3)$}

This model has $q=1, P=3$, and $\mathcal{D}_{2}=2$ [53]; thus, the number of variables is

$$
\left(1+q+2+P \cdot \mathcal{D}_{q+1}\right)_{q=1, P=3}=1+3+6=10,
$$

and the corresponding variables split as $s, x^{0}, x^{1}, x^{2}, y^{1} \ldots, y^{6}$; thus, in this model $I=0,1,2$ and $\alpha=1, \ldots, 6$. This is one of the simplest models related to a homogeneous non-symmetric 'special' manifold, and for which, to the best of our knowledge, the BPS black hole entropy and attractors are not known in literature. Therefore, In appendix B we work out the computations of this model in full detail.

\section{$10.7 L(1, P)$}

Some models of type $L(1, P)$ have been treated explicitly: $L(1,1)$ in section $8.2 .7, L(1,2)$ in section 9.5, and $L(1,3)$ in section 10.6 (see also the discussion of $L(1,4)$ and $L(1,8)$ in section 11 below). As far as we currently understand, for $P \geqslant 9$ such models cannot be obtained as descendants of other complete models, so in section 10.7.1 we will discuss the class of models $L(1, P)$ with $P \geqslant 2$, following the approach of section 10.2 , and retrieving (for $P=2$ resp. 3 ) the explicit treatment of the models $L(1,2)$ and $L(1,3)$, respectively given in sections 9.5 and 10.6. Moreover, in section 10.7.2 we will also briefly present a geometric point of view on the factorization of the inverse map of the gradient map, whose detailed investigation goes beyond the scope of this paper.

\subsection{1 $P \geqslant 2$}

By definition, $\mathcal{D}_{2}=2[53]$; thus, the number of variables is

$$
\left(1+q+2+P \cdot \mathcal{D}_{q+1}\right)_{q=1, P \geqslant 2}=1+3+2 P=2 P+4 .
$$

The cubic form $\mathcal{V}_{L(1, P)}$ reads

$$
\mathcal{V}_{L(1, P)}=s q(x)+\sum_{I=0}^{2} x^{I} Q_{I}^{(P)}(y), \quad \text { with } \quad q(x)=-\left(x^{0}\right)^{2}+\left(x^{1}\right)^{2}+\left(x^{2}\right)^{2}
$$

where the $Q_{I}^{(P)}$ are obtained from the $Q_{I}$ 's of the $L(1,1)$ model (cfr. (8.70)):

$$
Q_{0}^{(P)}=\sum_{i=1}^{2 P}\left(y^{i}\right)^{2} ; \quad Q_{1}^{(P)}=\sum_{i=1}^{2 P}(-1)^{i+1}\left(y^{i}\right)^{2} ; \quad Q_{2}^{(P)}=2\left(\sum_{j=1}^{P} y^{2 j-1} y^{2 j}\right) \text {. }
$$


Moreover, we define the quadrics, based on the quadric $R$ of the $L(1,2)$ model (see (A.3)), $R^{(k, l)}(y):=R\left(y^{(k l)}\right)$ for every $(k, l)$ with $1 \leqslant k<l \leqslant P$ :

$$
R^{(k, l)}=2\left(y^{k} y^{2 l}-y^{k+1} y^{2 l-1}\right),
$$

which depend only on the four variables $y^{k}, y^{k+1}, y^{2 l-1}, y^{2 l}$.

As required by the invertibility condition of section 6.2 (cfr. (6.8)), a Lorentzian identity of type (10.4) holds true.

Since $r=\left(\begin{array}{c}P \\ 2\end{array}\right)$, the inverse of the gradient map $\nabla_{\mathcal{V}_{L(1, P)}}$ is given as a composition of two maps, namely (cfr. (4.41))

$$
\mathbb{R}_{\xi}^{1+3+2 P} \stackrel{\nabla \mathcal{V}_{L(1, P)}}{\longrightarrow} \mathbb{R}_{z}^{1+3+2 P} \stackrel{\alpha}{\longrightarrow} \mathbb{R}_{(t, u, v, w)}^{1+3+2 P+\left(\begin{array}{c}
P \\
2
\end{array}\right)} \stackrel{\mu}{\longrightarrow} \mathbb{R}_{\xi}^{1+3+2 P},
$$

with

$$
\mu \circ \alpha \circ \nabla_{\mathcal{V}_{L(1, P)}}(\xi)=4 q(x)^{2} \mathcal{V}_{L(1, P)}(\xi) \xi
$$

where $\xi={ }^{T}(s, x, y)$, and $\mathcal{V}_{L(1, P)}$ and the corresponding $q(x)$ are given by (10.36). The map $\alpha$ from (6.14) is given by

$$
\alpha\left(z_{1}, \ldots, z_{2 P+4}\right):={ }^{T}\left(z_{1}^{2}, z_{1} z_{2}, \ldots, z_{1} z_{2 P+4}, \ldots, R^{(k, l)}(z), \ldots\right),
$$

where each of the $\left(\begin{array}{l}P \\ 2\end{array}\right)$ quadratic forms with $1 \leq k<l \leq P$ :

$$
R^{(k, l)}(z)=R\left(z_{4+2 k-1}, z_{4+2 k}, z_{4+2 l-1}, z_{4+2 l}\right)=2\left(z_{4+2 k-1} z_{4+2 l}-z_{4+2 l-1} z_{4+2 k}\right)
$$

depends only on four of the last $2 P$ variables $z 5, \ldots, z_{2 P+4}$.

Next, the map $\mu$ from (6.17), which has $2 P+4$ components that are homogeneous polynomials of degree 2 in the variables $t, u_{0}, u_{1}, u_{2}, v_{1}, \ldots, v_{2 P}, \ldots w_{k, l} \ldots$ with $1 \leqslant k<$ $l \leqslant P$, is given by

$$
\mu(t, u, v, w):=\left(\begin{array}{c}
\mu: \mathbb{R}_{t, u, v, w}^{1+3+2 P+\left(\begin{array}{l}
P \\
2
\end{array}\right)} \longrightarrow \mathbb{R}_{\xi}^{2 P+4}, \\
-u_{0}^{2}+u_{1}^{2}+u_{2}^{2}+\frac{1}{16}\left(w_{1,2}^{2}+\ldots+w_{P-1, P}^{2}\right) \\
-2 t u_{0}+\frac{1}{2}\left(v_{1}^{2}+v_{2}^{2}+\ldots+v_{2 P-1}^{2}+v_{2 P}^{2}\right) \\
2 t u_{1}+\frac{1}{16}\left(v_{1}^{2}-v_{2}^{2}+\ldots+v_{2 P-1}^{2}-v_{2 P}^{2}\right) \\
2 t u_{2}+v_{1} v_{2}+\ldots+v_{2 P-1} v_{2 P} \\
\left.u_{0} v_{1}+u_{1} v_{1}+u_{2} v_{2}-\frac{1}{4}\left(w_{1,2} v_{4}+w_{1,3} v_{6} \ldots+w_{1, P} v_{2 P}\right)\right) \\
u_{0} v_{2}-u_{1} v_{2}+u_{2} v_{1}-\frac{1}{4}\left(-w_{1,2} v_{3}-w_{1,3} v_{5} \ldots-w_{1, P} v_{2 P-1}\right) \\
\vdots \\
u_{0} v_{2 P-1}+u_{1} v_{2 P-1}+u_{2} v_{2 P}-\frac{1}{4}\left(-w_{1, P} v_{2}-w_{2, P} v_{4} \ldots-w_{P-1, P} v_{2 P-2}\right) \\
u_{0} v_{2 P}-u_{1} v_{2 P}+u_{2} v_{2 P-1}-\frac{1}{4}\left(w_{1, P} v_{1}+w_{2, P} v_{3} \ldots+w_{P-1, P} v_{2 P-3}\right)
\end{array}\right) .
$$

The (birational) inverse map $\left(\nabla_{\mathcal{V}_{L(1, P)}}\right)^{-1}=\mu \circ \alpha$ of the gradient map $\nabla_{\mathcal{V}_{L(1, P)}}$ reads

$$
\mu \circ \alpha: \mathbb{R}_{z}^{2 P+4} \stackrel{\alpha}{\longrightarrow} \mathbb{R}_{(t, u, v, w)}^{1+3+2 P+\left(\begin{array}{c}
P \\
2
\end{array}\right)} \stackrel{\mu}{\longrightarrow} \mathbb{R}_{\xi}^{2 P+4},
$$


whose expression for arbitrary $P \geqslant 2$ is the following (cfr. (6.27)):

$$
\begin{aligned}
& (\mu \circ \alpha)(z):= \\
& \left(\begin{array}{l}
z_{1}^{2}\left(-z_{2}^{2}+z_{3}^{2}+z_{4}^{2}\right)+\frac{1}{4} \sum_{g=2}^{P}\left(z_{2 g+1} z_{2 g+4}-z_{2 g+2} z_{2 g+3}\right)^{2} \\
z_{1}^{2}\left[-2 z_{1} z_{2}+\frac{1}{2} \sum_{g=1}^{P}\left(z_{2 g+3}^{2}+z_{2 g+4}^{2}\right)\right] \\
z_{1}^{2}\left[2 z_{1} z_{3}+\frac{1}{2} \sum_{g=1}^{P}\left(z_{2 g+3}^{2}-z_{2 g+4}^{2}\right)\right] \\
z_{1}^{2}\left(2 z_{1} z_{4}+\sum_{g=1}^{P} z_{2 g+3} z_{2 g+4}\right) \\
z_{1}^{2}\left(z_{2} z_{5}+z_{3} z_{5}+z_{4} z_{6}\right)-\frac{1}{2} z_{1} \sum_{g=2}^{P}\left(z_{5} z_{2 g+4}-z_{6} z_{2 g+3}\right) z_{2 g+4} \\
z_{1}^{2}\left(z_{2} z_{6}-z_{3} z_{6}+z_{4} z_{5}\right)+\frac{1}{2} z_{1} \sum_{g=2}^{P}\left(z_{5} z_{2 g+4}-z_{6} z_{2 g+3}\right) z_{2 g+3} \\
\vdots \\
z_{1}^{2}\left(z_{2} z_{2 P+3}+z_{3} z_{2 P+3}+z_{4} z_{2 P+4}\right)-\frac{1}{2} z_{1} \sum_{g=2}^{P}\left(-z_{2 g+1} z_{2 P+4}+z_{2 g+2} z_{2 P+3}\right) z_{2 g+2} \\
z_{1}^{2}\left(z_{2} z_{2 P+4}-z_{3} z_{2 P+4}+z_{4} z_{2 P+3}\right)+\frac{1}{2} z_{1} \sum_{g=2}^{P}\left(-z_{2 g+1} z_{2 P+4}+z_{2 g+2} z_{2 P+3}\right) z_{2 g+1}
\end{array}\right)
\end{aligned}
$$

Note that for $P=2$ and $P=3$ one respectively retrieves the results (A.13) and (B.14).

Consequently, from the treatment of section 6 , the full fledged expression of the solution (6.53) of the BPS system of $L(1, P)$ for arbitrary $P \geqslant 2$ is given by $\left(\Delta_{1} \equiv \Delta_{s}\right.$, and recall the definition $(2.3))$ :

$$
\begin{aligned}
s & =\frac{3}{2\left|\Delta_{s}\right|} \frac{\left[\Delta_{s}^{2}\left(-\Delta_{2}^{2}+\Delta_{3}^{2}+\Delta_{4}^{2}\right)+\frac{1}{4} \sum_{g=2}^{P}\left(\Delta_{2 g+1} \Delta_{2 g+4}-\Delta_{2 g+2} \Delta_{2 g+3}\right)^{2}\right]}{\sqrt{\left(\partial_{p} \Delta\right) \cdot(\mu \circ \alpha)\left(\partial_{p} \Delta\right)}} ; \\
x^{0} & =\frac{3}{2} \frac{\left|\Delta_{s}\right|\left[-2 \Delta_{s} \Delta_{2}+\frac{1}{2} \sum_{g=1}^{P}\left(\Delta_{2 g+3}^{2}+\Delta_{2 g+4}^{2}\right)\right]}{\sqrt{\left(\partial_{p} \Delta\right) \cdot(\mu \circ \alpha)\left(\partial_{p} \Delta\right)}} ; \\
x^{1} & =\frac{3}{2} \frac{\left|\Delta_{s}\right|\left[2 \Delta_{s} \Delta_{3}+\frac{1}{2} \sum_{g=1}^{P}\left(\Delta_{2 g+3}^{2}-\Delta_{2 g+4}^{2}\right)\right]}{\sqrt{\left(\partial_{p} \Delta\right) \cdot(\mu \circ \alpha)\left(\partial_{p} \Delta\right)}} ; \\
x^{2} & =\frac{3}{2} \frac{\left|\Delta_{s}\right|\left(2 \Delta_{s} \Delta_{4}+\sum_{g=1}^{P} \Delta_{2 g+3} \Delta_{2 g+4}\right)}{\sqrt{\left(\partial_{p} \Delta\right) \cdot(\mu \circ \alpha)\left(\partial_{p} \Delta\right)}} ; \\
y^{1} & =\frac{3 \operatorname{sgn}\left(\Delta_{s}\right)}{2} \frac{\left[\Delta_{s}\left(\Delta_{2} \Delta_{5}+\Delta_{3} \Delta_{5}+\Delta_{4} \Delta_{6}\right)-\frac{1}{2} \sum_{g=2}^{P}\left(\Delta_{5} \Delta_{2 g+4}-\Delta_{6} \Delta_{2 g+3}\right) \Delta_{2 g+4}\right]}{\sqrt{\left(\partial_{p} \Delta\right) \cdot(\mu \circ \alpha)\left(\partial_{p} \Delta\right)}} \\
y^{2} & =\frac{3 \operatorname{sgn}\left(\Delta_{s}\right)}{2} \frac{\left[\Delta_{s}\left(\Delta_{2} \Delta_{6}-\Delta_{3} \Delta_{6}+\Delta_{4} \Delta_{5}\right)+\frac{1}{2} \sum_{g=2}^{P}\left(\Delta_{5} \Delta_{2 g+4}-\Delta_{6} \Delta_{2 g+3}\right) \Delta_{2 g+3}\right]}{\sqrt{\left(\partial_{p} \Delta\right) \cdot(\mu \circ \alpha)\left(\partial_{p} \Delta\right)}} ;
\end{aligned}
$$




$$
\begin{gathered}
y^{2 P-1}=\frac{3 \operatorname{sgn}\left(\Delta_{s}\right)}{2} \frac{\left[\begin{array}{c}
\Delta_{s}\left(\Delta_{2} \Delta_{2 P+3}+\Delta_{3} \Delta_{2 P+3}+\Delta_{4} \Delta_{2 P+4}\right) \\
-\frac{1}{2} \sum_{g=2}^{P}\left(-\Delta_{2 g+1} \Delta_{2 P+4}+\Delta_{2 g+2} \Delta_{2 P+3}\right) \Delta_{2 g+2}
\end{array}\right]}{\sqrt{\left(\partial_{p} \Delta\right) \cdot(\mu \circ \alpha)\left(\partial_{p} \Delta\right)}} ; \\
y^{2 P}=\frac{3 \operatorname{sgn}\left(\Delta_{s}\right)}{2} \frac{\left[\begin{array}{c}
\Delta_{s}\left(\Delta_{2} \Delta_{2 P+4}-\Delta_{3} \Delta_{2 P+4}+\Delta_{4} \Delta_{2 P+3}\right) \\
+\frac{1}{2} \sum_{g=2}^{P}\left(-\Delta_{2 g+1} \Delta_{2 P+4}+\Delta_{2 g+2} \Delta_{2 P+3}\right) \Delta_{2 g+1}
\end{array}\right]}{\sqrt{\left(\partial_{p} \Delta\right) \cdot(\mu \circ \alpha)\left(\partial_{p} \Delta\right)}},
\end{gathered}
$$

where

$$
\begin{aligned}
& \left(\partial_{p} \Delta\right) \cdot(\mu \circ \alpha)\left(\partial_{p} \Delta\right)=\Delta_{s}^{3}\left(-\Delta_{2}^{2}+\Delta_{3}^{2}+\Delta_{4}^{2}\right)+\frac{1}{4} \Delta_{s} \sum_{g=2}^{P}\left(\Delta_{2 g+1} \Delta_{2 g+4}-\Delta_{2 g+2} \Delta_{2 g+3}\right)^{2} \\
& +\Delta_{s}^{2} \Delta_{2}\left[-2 \Delta_{s} \Delta_{2}+\frac{1}{2} \sum_{g=1}^{P}\left(\Delta_{2 g+3}^{2}+\Delta_{2 g+4}^{2}\right)\right] \\
& +\Delta_{s}^{2} \Delta_{3}\left[2 \Delta_{s} \Delta_{3}+\frac{1}{2} \sum_{g=1}^{P}\left(\Delta_{2 g+3}^{2}-\Delta_{2 g+4}^{2}\right)\right] \\
& +\Delta_{s}^{2} \Delta_{4}\left(2 \Delta_{s} \Delta_{4}+\sum_{g=1}^{P} \Delta_{2 g+3} \Delta_{2 g+4}\right) \\
& +\Delta_{s} \Delta_{5}\left[\Delta_{s}\left(\Delta_{2} \Delta_{5}+\Delta_{3} \Delta_{5}+\Delta_{4} \Delta_{6}\right)-\frac{1}{2} \sum_{g=2}^{P}\left(\Delta_{5} \Delta_{2 g+4}-\Delta_{6} \Delta_{2 g+3}\right) \Delta_{2 g+4}\right] \\
& +\Delta_{s} \Delta_{6}\left[\Delta_{s}\left(\Delta_{2} \Delta_{6}-\Delta_{3} \Delta_{6}+\Delta_{4} \Delta_{5}\right)+\frac{1}{2} \sum_{g=2}^{P}\left(\Delta_{5} \Delta_{2 g+4}-\Delta_{6} \Delta_{2 g+3}\right) \Delta_{2 g+3}\right] \\
& +\ldots \\
& +\Delta_{s} \Delta_{2 P+3}\left[\Delta_{s}\left(\Delta_{2} \Delta_{2 P+3}+\Delta_{3} \Delta_{2 P+3}+\Delta_{4} \Delta_{2 P+4}\right)\right. \\
& \left.-\frac{1}{2} \sum_{g=2}^{P}\left(-\Delta_{2 g+1} \Delta_{2 P+4}+\Delta_{2 g+2} \Delta_{2 P+3}\right) \Delta_{2 g+2}\right] \\
& +\Delta_{s} \Delta_{2 P+4}\left[\Delta_{s}\left(\Delta_{2} \Delta_{2 P+4}-\Delta_{3} \Delta_{2 P+4}+\Delta_{4} \Delta_{2 P+3}\right)\right. \\
& \left.+\frac{1}{2} \sum_{g=2}^{P}\left(-\Delta_{2 g+1} \Delta_{2 P+4}+\Delta_{2 g+2} \Delta_{2 P+3}\right) \Delta_{2 g+1}\right] .
\end{aligned}
$$

Then, (6.66) and (6.73)-(6.75) respectively yield the corresponding full fledged expression of the BPS black hole entropy and of the BPS attractors:

$$
\frac{S}{\pi}=\frac{1}{3\left|p^{0}\right|} \sqrt{3 \frac{\left(\partial_{p} \Delta\right) \cdot(\mu \circ \alpha)\left(\partial_{p} \Delta\right)}{\Delta_{s}^{2}}-9\left[p^{0}(p \cdot q)-2 I_{3}(p)\right]^{2}}
$$




$$
\begin{aligned}
& z_{H}^{1}(\mathcal{Q})=\frac{3}{2} \frac{\left[\Delta_{s}^{2}\left(-\Delta_{2}^{2}+\Delta_{3}^{2}+\Delta_{4}^{2}\right)+\frac{1}{4} \sum_{g=2}^{P}\left(\Delta_{2 g+1} \Delta_{2 g+4}-\Delta_{2 g+2} \Delta_{2 g+3}\right)^{2}\right]}{\left(\partial_{p} \Delta\right) \cdot(\mu \circ \alpha)\left(\partial_{p} \Delta\right)} . \\
& \cdot\left[\frac{p^{0}(p \cdot q)-2 I_{3}(p)}{p^{0}}-\mathbf{i} \frac{3}{2} \frac{S}{\pi}\right]+\frac{p^{1}}{p^{0}} ; \\
& z_{H}^{2}(\mathcal{Q})=\frac{3}{2} \frac{\Delta_{s}^{2}\left[-2 \Delta_{s} \Delta_{2}+\frac{1}{2} \sum_{g=1}^{P}\left(\Delta_{2 g+3}^{2}+\Delta_{2 g+4}^{2}\right)\right]}{\left(\partial_{p} \Delta\right) \cdot(\mu \circ \alpha)\left(\partial_{p} \Delta\right)} \text {. } \\
& \cdot\left[\frac{p^{0}(p \cdot q)-2 I_{3}(p)}{p^{0}}-\mathbf{i} \frac{3}{2} \frac{S}{\pi}\right]+\frac{p^{2}}{p^{0}} \\
& z_{H}^{3}(\mathcal{Q})=\frac{3}{2} \frac{\Delta_{s}^{2}\left[2 \Delta_{s} \Delta_{3}+\frac{1}{2} \sum_{g=1}^{P}\left(\Delta_{2 g+3}^{2}-\Delta_{2 g+4}^{2}\right)\right]}{\left(\partial_{p} \Delta\right) \cdot(\mu \circ \alpha)\left(\partial_{p} \Delta\right)} \text {. } \\
& \cdot\left[\frac{p^{0}(p \cdot q)-2 I_{3}(p)}{p^{0}}-\mathbf{i} \frac{3}{2} \frac{S}{\pi}\right]+\frac{p^{3}}{p^{0}} \\
& z_{H}^{4}(\mathcal{Q})=\frac{3}{2} \frac{\Delta_{s}^{2}\left(2 \Delta_{s} \Delta_{4}+\sum_{g=1}^{P} \Delta_{2 g+3} \Delta_{2 g+4}\right)}{\left(\partial_{p} \Delta\right) \cdot(\mu \circ \alpha)\left(\partial_{p} \Delta\right)} . \\
& \cdot\left[\frac{p^{0}(p \cdot q)-2 I_{3}(p)}{p^{0}}-\mathbf{i} \frac{3}{2} \frac{S}{\pi}\right]+\frac{p^{4}}{p^{0}} \\
& z_{H}^{5}(\mathcal{Q})=\frac{3}{2} \frac{\Delta_{s}\left[\Delta_{s}\left(\Delta_{2} \Delta_{5}+\Delta_{3} \Delta_{5}+\Delta_{4} \Delta_{6}\right)-\frac{1}{2} \sum_{g=2}^{P}\left(\Delta_{5} \Delta_{2 g+4}-\Delta_{6} \Delta_{2 g+3}\right) \Delta_{2 g+4}\right]}{\left(\partial_{p} \Delta\right) \cdot(\mu \circ \alpha)\left(\partial_{p} \Delta\right)} . \\
& \cdot\left[\frac{p^{0}(p \cdot q)-2 I_{3}(p)}{p^{0}}-\mathbf{i} \frac{3}{2} \frac{S}{\pi}\right]+\frac{p^{5}}{p^{0}} ; \\
& z_{H}^{6}(\mathcal{Q})=\frac{3}{2} \frac{\Delta_{s}\left[\Delta_{s}\left(\Delta_{2} \Delta_{6}-\Delta_{3} \Delta_{6}+\Delta_{4} \Delta_{5}\right)+\frac{1}{2} \sum_{g=2}^{P}\left(\Delta_{5} \Delta_{2 g+4}-\Delta_{6} \Delta_{2 g+3}\right) z_{2 g+3}\right]}{\left(\partial_{p} \Delta\right) \cdot(\mu \circ \alpha)\left(\partial_{p} \Delta\right)} . \\
& \cdot\left[\frac{p^{0}(p \cdot q)-2 I_{3}(p)}{p^{0}}-\mathbf{i} \frac{3}{2} \frac{S}{\pi}\right]+\frac{p^{6}}{p^{0}} ; \\
& z_{H}^{2 P+3}(\mathcal{Q})=\frac{3}{2} \frac{\Delta_{s}\left[\begin{array}{c}
\Delta_{s}\left(\Delta_{2} \Delta_{2 P+3}+\Delta_{3} \Delta_{2 P+3}+\Delta_{4} \Delta_{2 P+4}\right) \\
-\frac{1}{2} \sum_{g=2}^{P}\left(-\Delta_{2 g+1} \Delta_{2 P+4}+\Delta_{2 g+2} \Delta_{2 P+3}\right) \Delta_{2 g+2}
\end{array}\right]}{\left(\partial_{p} \Delta\right) \cdot(\mu \circ \alpha)\left(\partial_{p} \Delta\right)} . \\
& \cdot\left[\frac{p^{0}(p \cdot q)-2 I_{3}(p)}{p^{0}}-\mathbf{i} \frac{3}{2} \frac{S}{\pi}\right]+\frac{p^{2 P+3}}{p^{0}} ; \\
& z_{H}^{2 P+4}(\mathcal{Q})=\frac{3}{2} \frac{\Delta_{s}\left[\begin{array}{c}
\Delta_{s}\left(\Delta_{2} \Delta_{2 P+4}-\Delta_{3} \Delta_{2 P+4}+\Delta_{4} \Delta_{2 P+3}\right) \\
+\frac{1}{2} \sum_{g=2}^{P}\left(-\Delta_{2 g+1} \Delta_{2 P+4}+\Delta_{2 g+2} \Delta_{2 P+3}\right) \Delta_{2 g+1}
\end{array}\right]}{\left(\partial_{p} \Delta\right) \cdot(\mu \circ \alpha)\left(\partial_{p} \Delta\right)} \\
& \cdot\left[\frac{p^{0}(p \cdot q)-2 I_{3}(p)}{p^{0}}-\mathbf{i} \frac{3}{2} \frac{S}{\pi}\right]+\frac{p^{2 P+4}}{p^{0}},
\end{aligned}
$$


with $\left(\partial_{p} \Delta\right) \cdot(\mu \circ \alpha)\left(\partial_{p} \Delta\right)$ given by $(10.55)$, and

$$
\begin{aligned}
p \cdot q= & p^{0} q_{0}+p^{1} q_{1}+\ldots+p^{2 P+4} q_{2 P+4} \\
I_{3}(p)= & \mathcal{V}_{L(1, P)}\left(p^{1}, \ldots, p^{2 P+4}\right)=p^{1}\left[-\left(p^{2}\right)^{2}+\left(p^{3}\right)^{2}+\left(p^{4}\right)^{2}\right] \\
& +p^{2} Q_{0}^{(P)}\left(p^{5}, \ldots, p^{2 P+4}\right)+p^{3} Q_{1}^{(P)}\left(p^{5}, \ldots, p^{2 P+4}\right)+p^{4} Q_{2}^{(P)}\left(p^{5}, \ldots, p^{2 P+4}\right) \\
= & p^{1}\left[-\left(p^{2}\right)^{2}+\left(p^{3}\right)^{2}+\left(p^{4}\right)^{2}\right]+p^{2} \sum_{i=1}^{2 P}\left(p^{i+4}\right)^{2} \\
& +p^{3} \sum_{i=1}^{2 P}(-1)^{i+1}\left(p^{i+4}\right)^{2}+2 p^{4} \sum_{i=1}^{P} p^{2 i+3} p^{2 i+4}
\end{aligned}
$$

Consistently, it should be remarked that for $P=2$ and $P=3$ the above formulæ allow one to retrieve the explicit results obtained in sections 9.5 and 10.6, respectively.

It is here worth remarking that the above expressions provide, for the first time to the best of our knowledge, the explicit form of the BPS black hole entropy and attractors in an infinite class of (homogeneous) non-symmetric models of $\mathcal{N}=2, D=4$ supergravity with cubic prepotential.

\subsubsection{On the geometry of $\left(\nabla_{\mathcal{V}_{L(1, P)}}\right)^{-1}, P \geqslant 1$}

We will now describe briefly some properties of the factorization

$$
\left(\nabla_{\mathcal{V}_{L(1, P)}}\right)^{-1}=\mu \circ \alpha, P \geqslant 1
$$

in order to have a different, geometric perspective on its nature and on the arising of the quadratic forms $R_{k}$ 's (or $R_{K}^{(k, l)}$ 's) appearing in the Lorentzian quadratic identities (6.8), (9.7), or (10.4).

Since the map $\alpha: \mathbb{R}^{2 P+4} \rightarrow \mathbb{R}^{2 P+4+\left(\begin{array}{c}P \\ 2\end{array}\right)}$ is defined by homogenous polynomials of degree

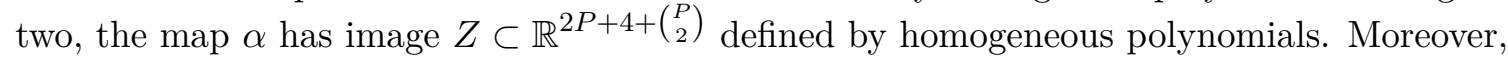
the dimension of $Z$, as an algebraic variety, is $2 P+4$, as it is easily seen by looking at the expression of $\alpha$. From the parametrization $\alpha$ of $Z$, we can deduce that it is a cone over a Grassmann variety $G(2, P+2)$. Moreover, the inverse of $\alpha$ (as a birational map from $\mathbb{R}^{2 P+4}$ to $\left.Z\right)$ is the restriction to $Z$ of the projection $\pi(t, u, v, w)={ }^{T}(t, u, v)$. Indeed, the map $\alpha: \mathbb{R}^{2 P+4} \rightarrow Z$ is such that, letting $z={ }^{T}\left(z_{1}, \ldots, z_{2 P+4}\right)$,

$$
(\pi \circ \alpha)(z)=z_{1} z
$$

The map $\mu: \mathbb{R}^{2 P+4+\left(\begin{array}{c}P \\ 2\end{array}\right)} \rightarrow \mathbb{R}^{2 P+4}$ restricted to $Z$ induces a map $\mu: Z \rightarrow \mathbb{R}^{2 P+4}$. The inverse (as a birational map) is the map $\phi: \mathbb{R}^{2 P+4} \rightarrow Z \subset \mathbb{R}^{2 P+4+\left(\begin{array}{c}P \\ 2\end{array}\right)}$ defined by

$$
\phi(\xi):={ }^{T}\left(\nabla_{\mathcal{V}_{L(1, P)}}(\xi), \ldots,-4 R^{(k, l)}(\xi), \ldots\right)
$$

because

$$
(\mu \circ \phi)(\xi)=4 \mathcal{V}_{L(1, P)}(\xi) \xi
$$


Indeed,

$$
q(x) \phi(\xi)=\alpha\left(\mathcal{V}_{L(1, P)}(\xi)\right)
$$

and

$$
(\mu \circ \alpha)\left(\mathcal{V}_{L(1, P)}(\xi)\right)=4 q(x)^{2} \mathcal{V}_{L(1, P)}(\xi) \xi
$$

A similar description of the images $Z$ 's of the corresponding maps $\alpha$ 's and the definition of the corresponding $\phi$ 's can actually be provided for all the models $L(q, P)$ treated until now; however, a thorough treatment goes well beyond the scope of this paper, and we leave it for further future work.

This geometric approach has many interesting applications for the study of the geometry of the singular locus of the cubic hypersurface $\mathcal{V}_{L(q, P)}$ in the associated projective space, which in many cases is a notable algebraic variety (for example in the complete case, but not only). In some cases, the quadratic homogeneous polynomials defining the map $\phi$ are a basis of all homogenous quadratic polynomials vanishing on (some irreducible component of) the singular locus of the cubic. It may also happen that there are more quadratic equations vanishing on the singular locus than the partial derivatives of $\mathcal{V}_{L(q, P)}$ which define it, and this explains why correspondingly $r>0$. This geometric point of view and its applications to Algebraic Geometry will be considered elsewhere.

\section{Non-uniqueness of $\Omega$ 's}

The models $L(1, P)$ for $P \geqslant 4$ can be handled with the general results obtained in section 10.2, as outlined in the previous section. However, those with $P \leqslant 8$ can also be embedded into the complete $L(8,1)$ model, and this provides an alternative way to determine the inverse of their gradient maps.

In this respect, we should stress the fact that the extra $\Omega$-matrices, required for noncomplete systems by the invertibility condition enounced in section 6.2 , may not be unique; even the number $r$ of such matrices is not determined uniquely by the model.

We illustrate this for $P=4$ and 8 , respectively in sections 11.1 and 11.2.

\section{$11.1 \quad L(1,4) \subset L(4,1)$}

The $L(1,4)$ model has $q=1, P=4$, and $\mathcal{D}_{2}=2$ [53]; thus, the number of variables is

$$
\left(1+q+2+P \cdot \mathcal{D}_{q+1}\right)_{q=1, P=4}=1+3+8=12 .
$$

The cubic form $\mathcal{V}_{L(1,4)}$ in the variables $s, x^{0}, x^{1}, x^{2}, y^{1}, \ldots, y^{8}$ reads

$$
\mathcal{V}_{L(1,4)}=s q(x)+\sum_{I=0}^{2} x^{I} Q_{I}^{(4)}(y), \quad \text { with } \quad q(x)=-\left(x^{0}\right)^{2}+\left(x^{1}\right)^{2}+\left(x^{2}\right)^{2}
$$

where the $Q_{I}^{(4)}$ 's are obtained from the $Q_{I}$ 's in the $L(1,1)$ model (cfr. (8.70)):

$$
\begin{aligned}
& Q_{0}^{(4)}=\left(y^{1}\right)^{2}+\left(y^{2}\right)^{2}+\left(y^{3}\right)^{2}+\left(y^{4}\right)^{2}+\left(y^{5}\right)^{2}+\left(y^{6}\right)^{2}+\left(y^{7}\right)^{2}+\left(y^{8}\right)^{2} \\
& Q_{1}^{(4)}=\left(y^{1}\right)^{2}-\left(y^{2}\right)^{2}+\left(y^{3}\right)^{2}-\left(y^{4}\right)^{2}+\left(y^{5}\right)^{2}-\left(y^{6}\right)^{2}+\left(y^{7}\right)^{2}-\left(y^{8}\right)^{2} \\
& Q_{2}^{(4)}=2\left(y^{1} y^{2}+y^{3} y^{4}+y^{5} y^{6}+y^{7} y^{8}\right) .
\end{aligned}
$$


In order to invert the gradient map of $\mathcal{V}_{L(1,4)}$, according to the condition in section 6.2 we need extra quadrics $R_{K}$ defined by matrices $\Omega_{K}$ satisfying a Lorentzian quadratic identity.

- One way to do so is to follow section 10.2 and thus to define the quadrics, based on the quadric $R$ in the $L(1,2)$ model given in (A.3), $R^{(k, l)}(y):=R\left(y^{(k l)}\right)$ :

$$
\begin{array}{ll}
R^{(1,2)}=2\left(y^{1} y^{4}-y^{2} y^{3}\right), & R^{(1,3)}=2\left(y^{1} y^{6}-y^{2} y^{5}\right), \\
R^{(2,3)}=2\left(y^{3} y^{6}-y^{4} y^{5}\right), & R^{(1,4)}=2\left(y^{1} y^{8}-y^{2} y^{7}\right), \\
R^{(2,4)}=2\left(y^{3} y^{8}-y^{4} y^{7}\right), & R^{(3,4)}=2\left(y^{5} y^{8}-y^{6} y^{7}\right) .
\end{array}
$$

As shown in general in 10.3, a Lorentzian identity of type (10.4) holds true, namely,

$$
-Q_{0}^{(4)}(y)^{2}+Q_{1}^{(4)}(y)^{2}+Q_{2}^{(4)}(y)^{2}+\sum_{1 \leqslant k<l \leqslant 4} R^{(k, l)}(y)^{2}=0 .
$$

The $L(1,4)$ model is thus invertible with $r=6$.

- Another approach is to take the $L(4,1)$ model discussed in section 8.2 .4 (itself a linearly constrained $L(8,1)$ model: cfr. (8.38)-(8.39)). The first three quadrics $\bar{Q}_{0}, \bar{Q}_{1}, \bar{Q}_{2}$ of this model, as listed in (8.36), are almost equal to the $Q_{i}^{(4)}$ above, and they become equal if we change the signs in some of the variables: ${ }^{30}$

$$
Q_{i}^{(4)}\left(y^{1}, y^{2}, y^{3}, y^{4}, y^{5}, y^{6}, y^{7}, y^{8}\right)=\bar{Q}_{i}\left(y^{1}, y^{2},-y^{3}, y^{4}, y^{5}, y^{6},-y^{7}, y^{8}\right) \quad(i=0,1,2) .
$$

There are three more quadratic forms $\bar{Q}_{3}, \bar{Q}_{4}, \bar{Q}_{5}$ in the $L(4,1)$ model, and the Lorentzian identity (8.37) holds between the six quadrics of the $L(4,1)$ model. In fact, if we define three quadratic forms by

$$
R_{K}\left(y^{1}, y^{2}, y^{3}, y^{4}, y^{5}, y^{6}, y^{7}, y^{8}\right):=\bar{Q}_{K+2}\left(y^{1}, y^{2},-y^{3}, y^{4}, y^{5}, y^{6},-y^{7}, y^{8}\right) \quad(K=1,2,3),
$$

then we obtain the Lorentzian identity

$$
-Q_{0}^{(4)}(y)^{2}+Q_{1}^{(4)}(y)^{2}+Q_{2}^{(4)}(y)^{2}+R_{1}(y)^{2}+R_{2}(y)^{2}+R_{3}(y)^{2}=0 .
$$

This shows that the descendant relation $L(1,4) \subset L(4,1)$ holds, and, again, the condition in section 6.2 is again satisfied, but now with $r=3$.

\section{$11.2 L(1,8) \subset L(8,1)$}

A similar treatment shows that $L(1,8)$ is a descendant of $L(8,1): L(1,8) \subset L(8,1)$. Indeed, the first three of the ten quadrics in $L(8,1)$ are the $Q_{i}^{(8)}, i=0,1,2$, of the $L(1,8)$ model, upon substituting

$$
y^{\alpha} \longrightarrow-y^{\alpha} \text { for } \alpha=3,7,11,15 .
$$

- In order to satisfy the invertibility condition enounced in section 6.2 , one can use the $R^{(k, l)}, 1 \leqslant k<l \leqslant 8$, as defined in section 10.2, and in this case one has $r=\left(\begin{array}{l}8 \\ 2\end{array}\right)=28$.

\footnotetext{
${ }^{30}$ Notice that we did something similar in (A.4) in section 9.5 for the $L(1,2)$ model.
} 
- Alternatively, one can use the remaining seven quadrics $Q_{3}, \ldots, Q_{9}$ and after the substitution (11.13) one obtains seven quadrics $R_{K}, K=1, \ldots, 7$, which again imply the invertibility condition of section 6.2 to be satisfied, now with $r=7$.

Clearly, also the models $L(1, P)$ with $P=5,6,7$ can be handled in a similar way (setting the last $16-2 P$ variables $y^{\alpha}$ equal to zero). Notice that $L(1,5), L(1,6), L(1,7)$ and $L(1,8)$ are descendant, but not submodels, of $L(8,1)$; see the discussion below (section 11.3).

More in general, it holds that

$$
L(1, P) \subset L(8,1), 1 \leqslant P \leqslant 8 .
$$

The key feature is that for $q+1 \leqslant 9$ the $\Gamma$-matrices $\Gamma_{1}, \ldots, \Gamma_{q+1}$ of $L(8,1)$ define a representation of the Clifford algebra $C l(q+1,0)$ : thus, there always exists $L(q, P, \dot{P}) \subset$ $L(8,1)$, for appropriate $q, P$ and $\dot{P}$ with $(P+\dot{P}) \mathcal{D}_{q+1}=16$ (setting some of the $y^{\alpha}$ variables equal to zero allows one to lower $P, \dot{P})$.

\subsection{Descendant $\nrightarrow$ submodel}

In the treatment given above, we have discussed various cases in which a model $L(q, P, \dot{P})$ can be regarded as a model $L\left(q^{\prime}, P^{\prime}, \dot{P}^{\prime}\right)$ with a larger number of variables (namely, $q+$ $\left.(P+\dot{P}) \cdot \mathcal{D}_{q+1}<q^{\prime}+\left(P^{\prime}+\dot{P}^{\prime}\right) \cdot \mathcal{D}_{q^{\prime}+1}\right)$ with some linear constraints (and possibly with some renaming of variables). $L(q, P, \dot{P})$ has thus been defined as a descendant of $L\left(q^{\prime}, P^{\prime}, \dot{P}^{\prime}\right)$, denoted by

$$
L(q, P, \dot{P}) \subset L\left(q^{\prime}, P^{\prime}, \dot{P}^{\prime}\right),
$$

and this relation has been instrumental in proving the invertibility of the corresponding gradient map by using the invertibility condition enounced in section 6.2 . Here, we want to point out that (11.15) does not necessarily imply that $L(q, P, \dot{P})$ is a submodel of $L\left(q^{\prime}, P^{\prime}, \dot{P}^{\prime}\right)$ (while the converse is trivially true).

Indeed, we have to recall that each model $L(q, P, \dot{P})$ corresponds to a non-compact, Riemannian homogeneous ("special") manifold, or more rigorously to a triplet of quaternionic Kähler, special Kähler and real special manifolds, which can be coupled to MaxwellEinstein supergravity with 8 supersymmetries respectively in 3,4 or 5 Lorentzian space-time dimensions $[53,56]$ :

$$
\begin{aligned}
L(q, P, \dot{P}) & \Longleftrightarrow\left\{\begin{array}{l}
\frac{G_{3}}{H_{3}} \text { quat. Kähler, } \\
\frac{G_{4}}{H_{4}} \text { special Kähler, } \\
\frac{G_{5}}{H_{5}} \text { real special, }
\end{array}\right. \\
H_{i} & =\operatorname{mcs}\left(G_{i}\right), i=3,4,5, \\
\frac{G_{3}}{H_{3}} & \supsetneq \frac{G_{4}}{H_{4}} \supsetneq \frac{G_{5}}{H_{5}}, \\
\text { with } G_{3} & \supsetneq G_{4} \supsetneq G_{5} \text { and } H_{3} \supsetneq H_{4} \supsetneq H_{5},
\end{aligned}
$$

where 'mcs' stands for maximal compact subgroup. Therefore, a necessary (but generally not sufficient) condition for a model $L$ (associated to $\left.\frac{G_{i}}{H_{i}}, i=3,4,5\right)$ to be a submodel of a 
model $L^{\prime}$ (associated to $\frac{G_{i}^{\prime}}{H_{i}^{\prime}}, i=3,4,5$ ) is that the structure of the corresponding cosets is consistent with the immersions, namely that

$$
G_{i} \subsetneq G_{i}^{\prime}, H_{i} \subsetneq H_{i}^{\prime} \forall i=3,4,5 .
$$

A counterexample is provided by $(11.14)$ with $P=5$ : in fact, $L(1,5)$ is a descendant of $L(8,1)$, namely

$$
L(1,5) \subset L(8,1),
$$

but $L(1,5)$ is not ${ }^{31}$ a submodel of $L(8,1)$. In fact $[37,56]$ :

$$
\begin{aligned}
& L(1,5) \Longleftrightarrow G_{5, L(1,5)}=(\mathrm{SO}(2,1) \otimes \mathrm{SO}(5) \otimes \mathrm{SO}(1,1))_{0} \ltimes(\mathbf{2}, \mathbf{5})_{3 / 2} ;
\end{aligned}
$$

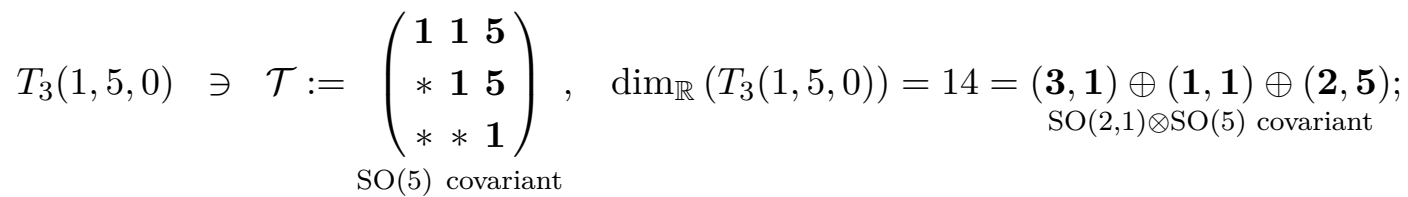

$$
\begin{aligned}
& L(8,1) \Longleftrightarrow G_{5, L(8,1)}=E_{6(-26)} \\
& J_{3}^{\mathbb{Q}} \ni \mathcal{J}:=\left(\begin{array}{ccc}
\mathbf{1} & \mathbf{8}_{v} & \mathbf{8}_{s} \\
* & \mathbf{1} & \mathbf{8}_{c} \\
* & * & \mathbf{1}
\end{array}\right), \quad \operatorname{dim}_{\mathbb{R}}\left(J_{3}^{\mathbb{Q}}\right)=27=\underset{E_{6(-26)} \text { covariant }}{\mathbf{2 7}}
\end{aligned}
$$

where $T_{3}(1,5,0)$ is the Hermitian part of a manifestly $\mathrm{SO}(5)$-covariant Vinberg's cubic T-algebra [135], and $J_{3}^{\mathbb{Q}}$ denotes the exceptional cubic Jordan algebra [86]. Note the Peirce decomposition of $J_{3}^{\mathbb{Q}}[7]$

$$
\begin{aligned}
& E_{6(-26)} \supsetneq \mathrm{SO}(9,1) \otimes \mathrm{SO}(1,1) ; \\
& \mathbf{2 7}=\mathbf{1}_{-4} \oplus \mathbf{1 0}_{2} \oplus \mathbf{1 6}_{-1} .
\end{aligned}
$$

Therefore, if the $L(1,5)$ model is a submodel $L(8,1)$ model, then it should hold, among other things, that

i)

$$
\begin{gathered}
G_{5, L(1,5)} \subsetneq G_{5, L(8,1)} \\
\stackrel{\Downarrow}{ } \\
(\mathrm{SO}(2,1) \otimes \mathrm{SO}(5) \otimes \mathrm{SO}(1,1))_{0} \ltimes(\mathbf{2}, \mathbf{5})_{3 / 2} \subsetneq \mathrm{SO}(9,1) \otimes \mathrm{SO}(1,1) \subsetneq E_{6(-26)} .
\end{gathered}
$$

ii) as an $\mathrm{SO}(9,1)$-representation (after Peirce decomposition), $J_{3}^{\mathbb{Q}}$ contains $T_{3}(1,5,0)$ (this latter as an $(\mathrm{SO}(2,1) \otimes \mathrm{SO}(5))$-representation).

By the results proven in appendix $\mathrm{C}$, it follows that the model $L(1,5)$ is not a submodel of $L(8,1)$, but rather only one of its descendants. It can thus be stated that the immersions giving rise to $L(1, P) \subset L(8,1), 5 \leqslant P \leqslant 8$, are not consistent with the structure of (the coset spaces respectively corresponding to) such models.

\footnotetext{
${ }^{31} A$ fortiori, the same holds for the models $L(1,6), L(1,7)$ and $L(1,8)$.
} 


\section{Beyond $L(q, P)$ models: $L(4,1,1)$}

We will now consider the unique model of the present paper having a non-vanishing $\dot{P}$ : the model $L(4,1,1)$.

As we have observed in section 8.2.4, the complete model $L(4,1)$ can be obtained from the complete model $L(8,1)$ by setting $x^{6}=\ldots=x^{9}=0$ and by taking the upper left $8 \times 8$ block $\bar{\Gamma}_{I}$ of the first six $\Gamma$-matrices $\Gamma_{0}, \ldots, \Gamma_{5}$ (of size $\left.16 \times 16\right)$ of $L(8,1)$. A closer look at such six $\Gamma$-matrices reveals that $(I=0,1,3,4,5)$ :

$$
\Gamma_{I}=\bar{\Gamma}_{I} \otimes \mathbb{I}_{2}=\left(\begin{array}{cc}
\bar{\Gamma}_{I} & 0 \\
0 & \bar{\Gamma}_{I}
\end{array}\right), \quad \Gamma_{2}=\bar{\Gamma}_{2} \otimes \sigma_{3}=\left(\begin{array}{cc}
\bar{\Gamma}_{2} & 0 \\
0 & -\bar{\Gamma}_{2}
\end{array}\right) .
$$

The representation $\psi: C l(5,0) \rightarrow M_{16}(\mathbb{R})$ defined by $\psi\left(e_{I}\right):=\Gamma_{I}, I=1, \ldots, 5$, is thus reducible, and it is the direct sum of the two non-equivalent irreducible 8-dimensional representations of $C l(5,0)$, since the sign of only one $\bar{\Gamma}_{I}$ is changed in the second component (cfr. section 5.3).

By recalling the discussion in section 4.3, one can then conclude that $L(4,1,1) \subset L(8,1)$ : by using the first $1+5 \Gamma$-matrices of $L(8,1)$, one indeed obtains

$$
L(4,1,1)=\left.L(8,1)\right|_{x^{6}=\ldots=x^{9}=0},
$$

so $L(4,1,1)$ can be regarded as the $L(8,1)$ model with four linear constraints. The $L(4,1,1)$ model has $q=4, P=\dot{P}=1$, and $\mathcal{D}_{5}=8$ [53], so the number of variables is

$$
\left(1+q+2+(P+\dot{P}) \cdot \mathcal{D}_{q+1}\right)_{q=4, P=1, \dot{P}=1}=1+6+(1+1) \cdot 8=23 .
$$

With the notation from (12.7) below, the cubic form of this model is

$$
\mathcal{V}_{L(4,1,1)}:=s q(x)+\sum_{I=0}^{5} x^{I} Q_{I}^{(1,1)}(y)=\left.\mathcal{V}_{L(8,1)}\right|_{x^{6}=\ldots=x^{9}=0},
$$

where

$$
q(x)=-\left(x^{0}\right)^{2}+\left(x^{1}\right)^{2}+\left(x^{2}\right)^{2}+\ldots+\left(x^{5}\right)^{2} .
$$

The remaining four $\Gamma$-matrices $\Gamma_{6}, \ldots, \Gamma_{9}$ of the $L(8,1)$ model are used as the extra matrices $\Omega_{1}, \ldots, \Omega_{4}$ occurring in the invertibility condition of section 6.2 (with $r=4$ ):

$$
\Omega_{K}=\Gamma_{5+K}, \quad \text { so } \quad R_{K}(y)=Q_{5+K}(y), \quad(K=1, \ldots, 4) .
$$

Therefore, the set of $\Gamma$-matrices $\left\{\Gamma_{1}, \ldots, \Gamma_{9}\right\}$ of $L(8,1)$ is a Clifford set and the anticommutativity conditions in the condition of section 6.2 are satisfied. The Lorentzian quadratic relation (8.5), where we now change the names according to (12.7), then shows that Lorentzian quadratic identity

$$
-Q_{0}^{(1,1)}(y)^{2}+Q_{1}^{(1,1)}(y)^{2}+\ldots+Q_{5}^{(1,1)}(y)^{2}+R_{1}(y)^{2}+\ldots+R_{4}(y)^{2}=0
$$

holds, as required by the aforementioned condition, which then implies that the gradient map of $L(4,1,1)$ is invertible. 
We now present the quadrics of the $L(8,1)$ system, as given in $(8.2)$, with the appropriate renamings suitable for the $L(4,1,1)$ model:

$$
\begin{aligned}
Q_{0}^{(1,1)} \equiv Q\left[\begin{array}{l}
0000 \\
0000
\end{array}\right]:=\left(y^{1}\right)^{2}+\ldots+\left(y^{8}\right)^{2}+\left(y^{9}\right)^{2}+\ldots+\left(y^{16}\right)^{2} ; \\
Q_{1}^{(1,1)} \equiv Q\left[\begin{array}{l}
0000 \\
0001
\end{array}\right]:=\left\{\begin{array}{l}
\left(y^{1}\right)^{2}-\left(y^{2}\right)^{2}+\ldots+\left(y^{7}\right)^{2}-\left(y^{8}\right)^{2} \\
+\left(y^{9}\right)^{2}-\left(y^{10}\right)^{2}+\ldots+\left(y^{15}\right)^{2}-\left(y^{16}\right)^{2} ;
\end{array}\right. \\
Q_{2}^{(1,1)} \equiv Q\left[\begin{array}{l}
0001 \\
1010
\end{array}\right]:=2\left(y^{1} y^{2}-y^{3} y^{4}+y^{5} y^{6}-y^{7} y^{8}-y^{9} y^{10}+y^{11} y^{12}-y^{13} y^{14}+y^{15} y^{16}\right) ; \\
Q_{3}^{(1,1)} \equiv Q\left[\begin{array}{l}
00000 \\
0000
\end{array}\right]:=2\left(y^{1} y^{4}+y^{2} y^{3}+y^{5} y^{8}+y^{6} y^{7}+y^{9} y^{12}+y^{10} y^{11}+y^{13} y^{16}+y^{14} y^{15}\right) ; \\
Q_{4}^{(1,1)} \equiv Q\left[\begin{array}{l}
0101 \\
0101
\end{array}\right]:=2\left(y^{1} y^{6}-y^{2} y^{5}+y^{3} y^{8}-y^{4} y^{7}+y^{9} y^{14}-y^{10} y^{13}+y^{11} y^{16}-y^{12} y^{15}\right) ; \\
Q_{5}^{(1,1)} \equiv Q\left[\begin{array}{l}
0111 \\
0110
\end{array}\right]:=2\left(y^{1} y^{8}+y^{2} y^{7}-y^{3} y^{6}-y^{4} y^{5}+y^{9} y^{16}+y^{10} y^{15}-y^{11} y^{14}-y^{12} y^{13}\right) ; \\
R_{1} \equiv Q\left[\begin{array}{l}
1001 \\
0010
\end{array}\right]:=2\left(y^{1} y^{10}+y^{2} y^{9}-y^{3} y^{12}-y^{4} y^{11}+y^{5} y^{14}+y^{6} y^{13}-y^{7} y^{16}-y^{8} y^{15}\right) ; \\
R_{2} \equiv Q\left[\begin{array}{l}
1011 \\
1101
\end{array}\right]:=2\left(y^{1} y^{12}-y^{2} y^{11}+y^{3} y^{10}-y^{4} y^{9}-y^{5} y^{16}+y^{6} y^{15}-y^{7} y^{14}+y^{8} y^{13}\right) ; \\
R_{3} \equiv Q\left[\begin{array}{l}
1101 \\
110
\end{array}\right]:=2\left(y^{1} y^{14}+y^{2} y^{13}-y^{3} y^{16}-y^{4} y^{15}-y^{5} y^{10}-y^{6} y^{9}+y^{7} y^{12}+y^{8} y^{11}\right) ; \\
R_{4} \equiv Q\left[\begin{array}{l}
1111 \\
1001
\end{array}\right]:=2\left(y^{1} y^{16}-y^{2} y^{15}+y^{3} y^{14}-y^{4} y^{13}+y^{5} y^{12}-y^{6} y^{11}+y^{7} y^{10}-y^{8} y^{9}\right) .
\end{aligned}
$$

Since $r=4$, the inverse of the gradient map $\nabla_{\mathcal{V}_{L(4,1,1)}}$ is given as a composition of two maps, namely (cfr. (4.41))

$$
\mathbb{R}_{\xi}^{1+6+16} \stackrel{\nabla_{\mathcal{V}_{L(1,3)}}^{\longrightarrow}}{\longrightarrow} \mathbb{R}_{z}^{1+6+16} \stackrel{\alpha}{\longrightarrow} \mathbb{R}_{(t, u, v, w)}^{1+6+16+4} \stackrel{\mu}{\longrightarrow} \mathbb{R}_{\xi}^{1+6+16}
$$

where $\xi=(s, x, y)$, and $\mathcal{V}_{L(4,1,1)}$ and the corresponding $q(x)$ are given by (12.4) and (12.5), respectively. The map $\alpha$ from (6.14) is given by

$$
\alpha\left(z_{1}, \ldots, z_{23}\right):={ }^{T}\left(z_{1}^{2}, z_{1} z_{2}, \ldots, z_{1} z_{23}, R_{1}(z), R_{2}(z), R_{3}(z), R_{4}(z)\right),
$$

where each of the three quadratic forms $R_{K}(z)$ 's $(K=1, \ldots, 4)$ depends only on four of the last $(1+1) \cdot 8=16$ variables:

$$
R_{K}(z)=R_{K}\left(z_{8}, \ldots, z_{23}\right)
$$

The map $\mu$ (6.17) has $1+3+16$ components that are homogeneous polynomials of degree 2 in the variables $t, u_{0}, u_{1}, u_{5}, v_{1}, \ldots, v_{16}, w_{1}, \ldots, w_{4}$. Since

$$
\mu \circ \alpha \circ \nabla_{\mathcal{V}_{L(4,1,1)}}(\xi)=4 q(x)^{2} \mathcal{V}_{L(4,1,1)}(\xi) \xi
$$

the (birational) inverse map $\left(\nabla_{\mathcal{V}_{L(4,1,1)}}\right)^{-1}=\mu \circ \alpha$ of the gradient map $\nabla_{\mathcal{V}_{L(4,1,1)}}$ is a homogeneous polynomial map of degree four. From the treatment of section 6 , the solution of the BPS system of $L(4,1,1)$ is then given by (6.54)-(6.56), and (6.66) and (6.73)(6.75) yield the corresponding expression of the BPS black hole entropy and of the BPS attractors, respectively. 


\section{Kleinian signatures and split algebras}

As already mentioned at the end of section 6.7 , one can also find sets of symmetric $\Gamma$ matrices defining a Clifford algebra representation of a quadratic form in $q+2=4,6,10$ dimensions with Kleinian "neutral" signatures $\left(2_{+}, 2_{-}\right),\left(3_{+}, 3_{-}\right)$and $\left(5_{+}, 5_{-}\right)$for $q=2,4$ and 8 respectively: these Kleinian signatures correspond to simple cubic Euclidean Jordan algebras over split composition algebras $J_{3}^{\mathbb{A}_{s}}$, for $\mathbb{A}=\mathbb{C}_{s}, \mathbb{H}_{s}$ and $\mathbb{O}_{s}$, respectively. These cases do not belong to the homogeneous special manifolds classified by $L(q, P, \dot{P})$ : in fact, they pertain to the so-called 'magic' non-supersymmetric Maxwell-Einstein theories, as well as to the maximal supergravity (in the case of split octonions $\mathbb{O}_{s}$ ); cfr. [101, 102]: they can be regarded as the 'Kleinian counterparts' of the magic $\mathcal{N}=2$ supergravity theories discussed in section 5 (recall (4.62) therein) [80, 101, 102]:

$$
\begin{array}{ccc}
J_{3}^{\mathbb{C}_{s}} & J_{3}^{\mathbb{H}_{s}} & J_{3}^{\mathbb{Q}_{s}} \\
\operatorname{dim} \mathbf{V}=1+4+4=9, & \operatorname{dim} \mathbf{V}=1+6+8=15, & \operatorname{dim} \mathbf{V}=1+10+16=27, \\
C l(1,2), & C l(2,3), & C l(4,5), \\
\mathcal{N}=0, & \mathcal{N}=0, & \mathcal{N}=8 .
\end{array}
$$

Notice that, as the magic $L(q, 1)$ models $(q=1,2,4,8)$ are related to Euclidean Clifford algebras $C l(q+1,0)$ (cfr. section 5), their 'Kleinian counterparts' models (existing for $q=2,4,8)$ are related to Clifford algebras $C l\left(\frac{q}{2}+1, \frac{q}{2}\right)$ in $q+1$ dimensions with (mostly minus) signature $\left(\frac{q}{2}+1, \frac{q}{2}\right)$; in fact, the reality properties of the spinors are the same for the $(q+1,0)$ and $\left(\frac{q}{2}+1, \frac{q}{2}\right)$ signatures in $q+1$ dimensions.

The extremal black hole entropy in maximal supergravity is explicitly known (see e.g. [62]), and by suitable truncations one obtains the same quantity in the $J_{3}^{\mathbb{H}_{s}}$ and $J_{3}^{\mathbb{C}_{s}}$ based theories.

For completeness's sake, we consider here the Kleinian model based on the exceptional cubic Jordan algebra $J_{3}^{\mathbb{Q}_{s}}$, which pertains to maximal supergravity; in this case,

$\mathcal{V}_{J_{3}^{\mathbb{S}_{S}}}=s q(x)+\sum_{I=1}^{10} x^{I} Q_{I}(y) \quad$ with $\quad q(x)=\left(x^{1}\right)^{2}+\ldots+\left(x^{5}\right)^{2}-\left(x^{6}\right)^{2}-\ldots-\left(x^{10}\right)^{2}$.

Thus, the quadratic form $q(x)$ has signature $(5,5)$, and the ten quadrics $Q_{I}$ 's read

$$
\begin{aligned}
Q_{1} & =2\left(y^{1} y^{6}-y^{2} y^{5}-y^{3} y^{8}+y^{4} y^{7}+y^{9} y^{14}-y^{10} y^{13}-y^{11} y^{16}+y^{12} y^{15}\right) ; \\
Q_{2} & =2\left(y^{1} y^{10}-y^{2} y^{9}+y^{3} y^{12}-y^{4} y^{11}-y^{5} y^{14}+y^{6} y^{13}-y^{7} y^{16}+y^{8} y^{15}\right) ; \\
Q_{3} & =2\left(y^{1} y^{11}-y^{2} y^{12}-y^{3} y^{9}+y^{4} y^{10}-y^{5} y^{15}+y^{6} y^{16}+y^{7} y^{13}-y^{8} y^{14}\right) ; \\
Q_{4} & =2\left(y^{1} y^{12}+y^{2} y^{11}-y^{3} y^{10}-y^{4} y^{9}-y^{5} y^{16}-y^{6} y^{15}+y^{7} y^{14}+y^{8} y^{13}\right) ; \\
Q_{5} & =2\left(y^{1} y^{14}-y^{2} y^{13}-y^{3} y^{16}+y^{4} y^{15}+y^{5} y^{10}-y^{6} y^{9}-y^{7} y^{12}+y^{8} y^{11}\right) ; \\
Q_{6} & =2\left(y^{1} y^{6}-y^{2} y^{5}-y^{3} y^{8}+y^{4} y^{7}-y^{9} y^{14}+y^{10} y^{13}+y^{11} y^{16}-y^{12} y^{15}\right) ; \\
Q_{7} & =2\left(y^{1} y^{10}-y^{2} y^{9}-y^{3} y^{12}+y^{4} y^{11}+y^{5} y^{14}-y^{6} y^{13}-y^{7} y^{16}+y^{8} y^{15}\right) ; \\
Q_{8} & =2\left(y^{1} y^{11}+y^{2} y^{12}-y^{3} y^{9}-y^{4} y^{10}-y^{5} y^{15}-y^{6} y^{16}+y^{7} y^{13}+y^{8} y^{14}\right) ; \\
Q_{9} & =2\left(y^{1} y^{12}-y^{2} y^{11}+y^{3} y^{10}-y^{4} y^{9}-y^{5} y^{16}+y^{6} y^{15}-y^{7} y^{14}+y^{8} y^{13}\right) ; \\
Q_{10} & =2\left(y^{1} y^{14}-y^{2} y^{13}-y^{3} y^{16}+y^{4} y^{15}-y^{5} y^{10}+y^{6} y^{9}+y^{7} y^{12}-y^{8} y^{11}\right) .
\end{aligned}
$$


These quadrics satisfy the Kleinian quadratic identity ${ }^{32}$

$$
q\left(Q_{1}, \ldots, Q_{10}\right)=Q_{1}^{2}+\ldots+Q_{5}^{2}-Q_{6}^{2}-\ldots-Q_{10}^{2}=0
$$

and the matrices defining these quadratic forms satisfy the Clifford relations (5.11). The differences between the model $L(8,1)$ treated in section 8.1 and the Kleinian model of maximal supergravity discussed here can be realized at a glance by comparing (8.3)-(8.4) and (8.2) to (13.2) and (13.3), respectively.

The inverse of the gradient map $\nabla_{\mathcal{V}_{J_{3}}^{\mathbb{O}_{S}}}$ can then be computed to be a polynomial map of degree two, similar to the one for the Lorenzian case discussed in section 8.1.

\section{Beyond the invertibility condition: $L(9,1)$}

Let us now consider the model $L(9,1)$.

The $q+2=11$ quadratic forms $Q_{0}, \ldots, Q_{10}$ are associated to $\Gamma_{0}=\mathbb{I}_{32}$ and to a set of $32 \times 32$ Clifford matrices $\left\{\Gamma_{1}, \ldots, \Gamma_{10}\right\}$, generating the Euclidean Clifford algebra ${ }^{33} \mathrm{Cl}(10,0)$. It is not hard to find such matrices: by denoting by $\Gamma_{1}^{\prime}, \ldots, \Gamma_{9}^{\prime}$ the $16 \times 16$ Clifford matrices of $C l(9,0)$ (which correspond to the quadrics $Q_{1}, \ldots, Q_{9}$ in the complete model $L(8,1)$ discussed in section 8.1), for the Euclidean Clifford algebra $C l(10,0)$, corresponding to the model $L(9,1)$, it simply suffices to take the corresponding block matrices of size $32 \times 32$, as follows:

$$
\Gamma_{0}=\mathbb{I}_{32}, \quad \Gamma_{I}:=\left(\begin{array}{cc}
\Gamma_{I}^{\prime} & 0 \\
0 & -\Gamma_{I}^{\prime}
\end{array}\right), \quad(I=1, \ldots, 9) \quad \Gamma_{10}:=\mathbb{I}_{16} \otimes \sigma_{1}=\left(\begin{array}{cc}
0 & \mathbb{I}_{16} \\
\mathbb{I}_{16} & 0
\end{array}\right)
$$

and one easily verifies that these matrices satisfy the Clifford relations (5.11).

Notice that there is no homomorphism $C l(11,0) \rightarrow M_{32}(\mathbb{R})$, so the Clifford set of $\Gamma$-matrices $\left\{\Gamma_{1}:=\mathbb{I}_{32}, \Gamma_{2}, \ldots, \Gamma_{10}\right\}$ is maximal for the size $32 \times 32$, namely one cannot add another $\Gamma$-matrix of size $32 \times 32$ and still have a Clifford set.

However, in striking contrast to the complete models related to $\Gamma$-matrices of size $m=2^{g}$ with $g=1,2,3,4$, discussed in section 6.7 and respectively treated in sections 8.2.7, 8.2.6, 8.2.4 and 8.1, there is no (Lorentzian) quadratic identity between the 11 quadratic forms $Q_{0}, \ldots, Q_{10}$. Therefore, one cannot exploit the invertibility condition enounced in section 6.2 in order to determine the invertibility of the gradient map $\nabla_{\mathcal{V}_{L(9,1)}}$. Of course, such a condition provides a sufficient but not necessary condition for invertibility, so the lack of a suitable quadratic identity of quadrics does not necessarily imply the non-invertibility of the gradient map of the corresponding cubic form.

At any rate, other approaches to prove invertibility or non-invertibility of the gradient map $\nabla_{\mathcal{V}_{L(9,1)}}$ should be found, but they are beyond the scope of the present investigation.

\footnotetext{
${ }^{32}$ Notice that $Q_{I}^{2}-Q_{I+5}^{2}($ for $I=1,2,3,4,5)$ doesn't have that many terms.

${ }^{33}$ Recall the non-trivial homomorphism $C l(10,0) \rightarrow M_{2}(\mathbb{R}) \otimes M_{16}(\mathbb{R})=M_{32}(\mathbb{R})$; cfr. (5.8).
} 


\section{Final remarks and outlook}

We have considered the issue to obtain an explicit expression of the attractor values of scalar fields as well as of the Bekenstein-Hawking entropy, of static, asymptotically flat, dyonic, BPS extremal black holes in ungauged $\mathcal{N}=2$ Maxwell-Einstein supergravity theories in four space-time dimensions, coupled to non-linear sigma models of scalar fields endowed with very special geometry; this class of theories encompasses all four-dimensional $\mathcal{N}=2$ theories which can be obtained as an $S^{1}$-compactification of five-dimensional minimal supergravity theories. After [122], this problem can be translated into the issue of solving certain algebraic inhomogeneous systems of degree two, named BPS systems.

Within the so-called 'very special' geometry (related to cubic holomorphic prepotentials), we have focused on homogeneous non-compact Riemannian spaces. For homogeneous symmetric spaces, which are related to (simple and semi-simple) cubic (Euclidean) Jordan algebras, the solution to the BPS system is explicitly known, as is the expression of the BPS entropy and attractors (cfr. [62], and refs. therein): they can be formulated only in terms of a unique quartic invariant ${ }^{34}$ polynomial in the black hole electric-magnetic charges. On the other hand, not much is known for the homogeneous non-symmetric spaces; in fact, to the best of our knowledge, only [51] and [6] briefly treated, within a different formalism, the models $L(1,2)$ and $L(2,2)$. Therefore, in the present investigation we have focussed on homogeneous non-symmetric very special geometry, which has been classified, in terms of Euclidean Clifford algebras, in [53].

In section 6.2 we have formulated a (sufficient, but not necessary) condition for the invertibility of the gradient map of the cubic form defining the homogeneous non-symmetric very special geometry (and thus for the resolution of the related BPS system): this condition requires the existence of a suitable Lorentzian quadratic identity involving the quadratic forms defined by the symmetric $\Gamma$-matrices of the corresponding Euclidean real Clifford algebra, as well as some other quadratic forms defined by symmetric auxiliary matrices denoted by $\Omega_{K}$. Subsequently, we have thus provided in section 6.4 an explicit expression for the (birational) inverse map of the gradient map of the models for which the invertibility condition holds; the inverse map is a homogeneous polynomial map of degree four. Then, in section 6.5, we have presented, within the assumption that the aforementioned condition holds true, a procedure for the explicit solution of the related BPS system, determining in section 6.6 an explicit formula for the BPS Bekenstein-Hawking entropy of extremal black holes, as well as for the attractor values of the scalar fields in such a background. It is also here worth remarking that the explicit solution of the BPS system is also relevant for the solution of the attractor equations in asymptotically AdS, dyonic, extremal $\frac{1}{4}$-BPS black holes of $\mathrm{U}(1)$ Fayet-Iliopoulos gauged Maxwell-Einstein $\mathcal{N}=2$ supergravity in four space-time dimensions [65, 79].

Besides the general treatment given in section 6 (within the validity of the invertibility condition enounced in section 6.2 and then discussed in section 7) as well as in section 10.7.1

\footnotetext{
${ }^{34}$ In [62], the treatment of the present paper corresponds to the manifestly ' $G_{6}$-invariant' formalism discussed in section 3.4 therein; see also [52].
} 
(for the models $L(1, P)$ with $P \geqslant 4$ ), we have explicitly considered various homogeneous non-symmetric models, namely:

- $L(q, 1), q=1,2, \ldots, 8$ (section 8$)$ and 9 (section 14$)$;

- $L(q, 2), q=1,2,3$ (section 9$)$;

- $L(q, P), q=1,2,3, P \geqslant 3$ (section 10), with explicit emphasis given to the models $L(1, P)$ with $P \geqslant 2$ given in section 10.7 ;

- $L(4,1,1)$ (section 12).

In particular, the models $L(1,2)$ and $L(1,3)$ have been worked out in full detail in sections 9.5 resp. 10.6, and in section 10.7 their treatment has been generalized (in a $P$-dependent manner) to the infinite class of $L(1, P) P \geqslant 2$ non-symmetric models of $\mathcal{N}=2$, $D=4$ supergravity. In this respect, we have extended the treatment given in section 4 of [122], by providing, for the first time to the best of our knowledge, the explicit form of the BPS black hole entropy and of the BPS attractors in an infinite class of (homogeneous) non-symmetric models of $\mathcal{N}=2$ supergravity with cubic prepotential.

Still, many homogeneous non-symmetric models remain to be investigated for what concerns the invertibility of the corresponding gradient map, and thus the solution to the corresponding BPS system, aiming at obtaining explicit expressions for the BekensteinHawking entropy of extremal BPS black holes as well as for the corresponding BPS attractor configurations of the scalar fields. From the classification of [53] (see also [55, 56]), these models belong to the infinite series

- $L(q, 1), q \geqslant 10$

- $L(q, 2), q \geqslant 4$;

- $L(q, P), q \geqslant 4, P \geqslant 3$;

- $L(-1, P), P \geqslant 1$ (the so-called 'non-Jordan symmetric sequence' [54]);

- $L(4 m, P, \dot{P})$, with $^{35} m \geqslant 0, P \geqslant 1$, and $\dot{P} \geqslant 1$ (excluding the model $L(4,1,1)$ ).

We leave the treatment of such classes to future work. It would also be interesting to investigate the invertibility of the gradient map, and thus the solution to the corresponding BPS system, of cubic forms associated to noteworthy classes of non-homogeneous spaces.

Also, we would like to recall that in section 10.7.2, we have briefly considered a geometric perspective on the factorized nature of the inverse map of the gradient map of the cubic forms pertaining to the models $L(1, P)$ with $P \geqslant 1$. We conjecture that this holds essentially true for any $L(q, P)$ model, thus providing an explanation to $r>0$ in non-complete models; in future works, it will be interesting to discuss this geometric point of view in detail, as well as to study various subsequent applications to Algebraic Geometry.

\footnotetext{
${ }^{35}$ The models $L(0, P, \dot{P})$ have been treated, within a different formalism, in [6] and [51].
} 
Within this research venue, it would be interesting to investigate the geometric aspects of the examples of non-homogeneous very special geometry discussed by Shmakova in section 4 of [122] (cfr. refs. therein, as well), as well as of the non-homogeneous 2-moduli cubic models in which non-trivial involutory matrices determining multiple attractor solutions exist [98, 103]; we leave these tasks for further future work.

Moreover, some time ago, after the seminal paper of Moore [99], an intriguing relationship was observed between Gauss's composition laws and the arithmetics of BPS black holes in string theory and supergravity. Remarkably, this relationship has been recently extended to include Bhargava's higher composition laws, closely related to various classes of black hole solutions appearing in string/M-theory [15, 31, 67]. We leave to further future work the investigation of the possibility of a further generalization and extension to BPS black holes in theories with homogeneous non-symmetric scalar manifolds.

Finally, we remark that the stratification of the $U$-duality orbits supporting extremal black hole attractors is well known for symmetric models of $\mathcal{N}=2, D=4$ Maxwell-Einstein supergravity, since the seminal paper [59] (see e.g. [16, 60], and also [14] for a recollection of results). It woud be of utmost interest to extend the known results to the infinite classes of homogeneous non-symmetric models, especially to those which cannot be obtained by suitable truncations of symmetric models. Some advances along such a venue are discussed in [12], and we leave the investigation of this issue for future work.

\section{A Details on $L(1,2)$}

The cubic form $\mathcal{V}_{L(1,2)}$ is given by

$$
\mathcal{V}_{L(1,2)}(s, x, y)=s q(x)+x^{0} Q_{0}^{(2)}(y)+x^{1} Q_{1}^{(2)}(y)+x^{2} Q_{2}^{(2)}(y),
$$

where

$$
q(x):=-\left(x^{0}\right)^{2}+\left(x^{1}\right)^{2}+\left(x^{2}\right)^{2}
$$

and the $Q_{I}^{(2)}$ 's are given by (A.3) below.

To write this model explicitly and to show the invertibility of the gradient map, we first show that $L(1,2) \subset L(2,1)$; to this aim, we start and observe that both of the models are defined by $4 \times 4 \Gamma$-matrices. Using the quadratic forms from section 8.2 .6 but substituting $y^{3}:=-y^{3}$, one finds that the first three of these forms are the $Q_{I}^{(2)}$ with the $Q_{I}$ from the $L(2,1)$ model and the remaining form is denoted by $R \equiv R_{1}$ :

$$
\begin{array}{rlrl}
\Gamma_{0}^{(2)} & =\mathbb{I}_{4}, & Q_{0}^{(2)} \equiv Q\left[\begin{array}{l}
00 \\
00
\end{array}\right]=\left(y^{1}\right)^{2}+\left(y^{2}\right)^{2}+\left(y^{3}\right)^{2}+\left(y^{4}\right)^{2} ; \\
\Gamma_{1}^{(2)}=\sigma_{3} \otimes \mathbb{I}_{2}, & Q_{1}^{(2)} \equiv Q\left[\begin{array}{l}
{[01} \\
01
\end{array}\right]=\left(y^{1}\right)^{2}-\left(y^{2}\right)^{2}+\left(y^{3}\right)^{2}-\left(y^{4}\right)^{2} ; \\
\Gamma_{2}^{(2)}=\sigma_{1} \otimes \mathbb{I}_{2}, & Q_{2}^{(2)} \equiv Q\left[\begin{array}{l}
01 \\
10
\end{array}\right]=2\left(y^{1} y^{2}+y^{3} y^{4}\right) ; \\
\Omega_{1}=\sigma_{1} \otimes \sigma_{1}, & R & \equiv Q\left[\begin{array}{ll}
11 \\
11
\end{array}\right]=2\left(y^{1} y^{4}-y^{2} y^{3}\right) .
\end{array}
$$

Thus, it holds that:

$$
\begin{aligned}
& L(1,2)=\left.L(2,1)\right|_{x^{3}=0, y^{3}:=-y^{3}} \\
& \mathcal{V}_{L(1,2)}=\left.\mathcal{V}_{L(2,1)}\right|_{x^{3}=0, y^{3}:=-y^{3}}
\end{aligned}
$$


Namely, the model $L(1,2)$ can be regarded as the $L(2,1)$ model with one linear constraint, upon the renaming $y^{3}:=-y^{3}$.

The model $L(1,2)$ is invertible since the matrices $\Gamma_{1}^{(2)}, \Gamma_{2}^{(2)}, \Omega_{1}$ anti-commute, the first two form a Clifford set and finally the $Q_{I}^{(2)}$ 's and the $R(y)$ are related by the following quadratic (Lorentzian) identity:

$$
-Q_{0}^{(2)}(y)^{2}+Q_{1}^{(2)}(y)^{2}+Q_{2}^{(2)}(y)^{2}+R(y)^{2}=0 .
$$

Therefore, the invertibility condition of section 6.2 is satisfied, and $\nabla_{\mathcal{V}_{L(1,2)}}$ is invertible.

Since $r=1$, the inverse of the gradient map $\nabla_{\mathcal{V}_{L(1,2)}}$ is given as a composition of two maps $\alpha$ and $\mu$ (cfr. (4.41)):

$$
\mathbb{R}_{\xi}^{1+3+4} \stackrel{\nabla \mathcal{V}_{L(1,2)}}{\longrightarrow} \mathbb{R}_{z}^{1+3+4} \stackrel{\alpha}{\longrightarrow} \mathbb{R}_{(t, u, v, w)}^{1+3+4+1} \stackrel{\mu}{\longrightarrow} \mathbb{R}_{\xi}^{1+3+4} .
$$

The map $\alpha$, as in (6.14), is given by

$$
\alpha\left(z_{1}, \ldots, z_{8}\right):={ }^{T}\left(z_{1}^{2}, z_{1} z_{2}, \ldots, z_{1} z_{8}, R(z)\right),
$$

where the quadratic form $R$ depends only on the last $2 \cdot 2=4$ variables:

$$
R(z)=R\left(z_{5}, \ldots, z_{8}\right)=2\left(z_{5} z_{8}-z_{6} z_{7}\right) .
$$

The map $\mu$ (6.17) has $1+3+4=8$ components that are homogeneous polynomials of degree 2 in the variables $t, u_{0}, \ldots, u_{2}, v_{1}, \ldots, v_{4}, w$, and it is given by

$$
\mu(t, u, v, w):=\left(\begin{array}{c}
-u_{0}^{2}+u_{1}^{2}+u_{2}^{2}+\frac{1}{16} w^{2} \\
-2 t u_{0}+\frac{1}{2}\left(v_{1}^{2}+v_{2}^{2}+v_{3}^{2}+v_{4}^{2}\right) \\
2 t u_{1}+\frac{1}{2}\left(v_{1}^{2}-v_{2}^{2}+v_{3}^{2}-v_{4}^{2}\right) \\
2 t u_{2}+v_{1} v_{2}+v_{3} v_{4} \\
u_{0} v_{1}+u_{1} v_{1}+u_{2} v_{2}-\frac{1}{4} v_{4} w \\
u_{0} v_{2}-u_{1} v_{2}+u_{2} v_{1}+\frac{1}{4} v_{3} w \\
u_{0} v_{3}+u_{1} v_{3}+u_{2} v_{4}+\frac{1}{4} v_{2} w \\
u_{0} v_{4}-u_{1} v_{4}+u_{2} v_{3}-\frac{1}{4} v_{1} w
\end{array}\right) .
$$

Since

$$
\mu \circ \alpha \circ \nabla_{\mathcal{V}_{L(1,2)}}(\xi)=4 q(x)^{2} \mathcal{V}_{L(1,2)}(\xi) \xi,
$$

where $\mathcal{V}_{L(1,2)}$ and the corresponding $q(x)$ are given by (A.1) and (A.2), the (birational) inverse of the gradient map $\nabla_{\mathcal{V}_{L(1,2)}}$ is the map

$$
\mu \circ \alpha: \mathbb{R}_{z}^{1+3+4} \stackrel{\alpha}{\longrightarrow} \mathbb{R}_{(t, u, v, w)}^{1+3+4+1} \stackrel{\mu}{\longrightarrow} \mathbb{R}_{\xi}^{1+3+4},
$$


given by the following homogeneous polynomials of degree four (cfr. (6.27)):

$$
(\mu \circ \alpha)(z):=\left(\begin{array}{l}
z_{1}^{2}\left(-z_{2}^{2}+z_{3}^{2}+z_{4}^{2}\right)+\frac{1}{4}\left(z_{5} z_{8}-z_{6} z_{7}\right)^{2} \\
z_{1}^{2}\left[-2 z_{1} z_{2}+\frac{1}{2}\left(z_{5}^{2}+z_{6}^{2}+z_{7}^{2}+z_{8}^{2}\right)\right] \\
z_{1}^{2}\left[2 z_{1} z_{3}+\frac{1}{2}\left(z_{5}^{2}-z_{6}^{2}+z_{7}^{2}-z_{8}^{2}\right)\right] \\
z_{1}^{2}\left(2 z_{1} z_{4}+z_{5} z_{6}+z_{7} z_{8}\right) \\
z_{1}\left[z_{1}\left(z_{2} z_{5}+z_{3} z_{5}+z_{4} z_{6}\right)-\frac{1}{2}\left(z_{5} z_{8}-z_{6} z_{7}\right) z_{8}\right] \\
z_{1}\left[z_{1}\left(z_{2} z_{6}-z_{3} z_{6}+z_{4} z_{5}\right)+\frac{1}{2}\left(z_{5} z_{8}-z_{6} z_{7}\right) z_{7}\right] \\
z_{1}\left[z_{1}\left(z_{2} z_{7}+z_{3} z_{7}+z_{4} z_{8}\right)+\frac{1}{2}\left(z_{5} z_{8}-z_{6} z_{7}\right) z_{6}\right] \\
z_{1}\left[z_{1}\left(z_{2} z_{8}-z_{3} z_{8}+z_{4} z_{7}\right)-\frac{1}{2}\left(z_{5} z_{8}-z_{6} z_{7}\right) z_{5}\right]
\end{array}\right) .
$$

Consequently, from the treatment of section 6 , the full fledged expression of the solution (6.53) of the BPS system of $L(1,2)$ is given by $\left(\Delta_{1} \equiv \Delta_{s}\right.$, and recall the definition (2.3)):

$$
\begin{aligned}
& s=\frac{3}{2\left|\Delta_{s}\right|} \frac{\left[\Delta_{s}^{2}\left(-\Delta_{2}^{2}+\Delta_{3}^{2}+\Delta_{4}^{2}\right)+\frac{1}{4}\left(\Delta_{5} \Delta_{8}-\Delta_{6} \Delta_{7}\right)^{2}\right]}{\sqrt{\left(\partial_{p} \Delta\right) \cdot(\mu \circ \alpha)\left(\partial_{p} \Delta\right)}} ; \\
& x^{0}=\frac{3}{2} \frac{\left|\Delta_{s}\right|\left[-2 \Delta_{s} \Delta_{2}+\frac{1}{2}\left(\Delta_{5}^{2}+\Delta_{6}^{2}+\Delta_{7}^{2}+\Delta_{8}^{2}\right)\right]}{\sqrt{\left(\partial_{p} \Delta\right) \cdot(\mu \circ \alpha)\left(\partial_{p} \Delta\right)}} ; \\
& x^{1}=\frac{3}{2} \frac{\left|\Delta_{s}\right|\left[2 \Delta_{s} \Delta_{3}+\frac{1}{2}\left(\Delta_{5}^{2}-\Delta_{6}^{2}+\Delta_{7}^{2}-\Delta_{8}^{2}\right)\right]}{\sqrt{\left(\partial_{p} \Delta\right) \cdot(\mu \circ \alpha)\left(\partial_{p} \Delta\right)}} ; \\
& x^{2}=\frac{3}{2} \frac{\left|\Delta_{s}\right|\left(2 \Delta_{s} \Delta_{4}+\Delta_{5} \Delta_{6}+\Delta_{7} \Delta_{8}\right)}{\sqrt{\left(\partial_{p} \Delta\right) \cdot(\mu \circ \alpha)\left(\partial_{p} \Delta\right)}} ; \\
& y^{1}=\frac{3 \operatorname{sgn}\left(\Delta_{s}\right)}{2} \frac{\left[\Delta_{s}\left(\Delta_{2} \Delta_{5}+\Delta_{3} \Delta_{5}+\Delta_{4} \Delta_{6}\right)-\frac{1}{2}\left(\Delta_{5} \Delta_{8}-\Delta_{6} \Delta_{7}\right) \Delta_{8}\right]}{\sqrt{\left(\partial_{p} \Delta\right) \cdot(\mu \circ \alpha)\left(\partial_{p} \Delta\right)}} ; \\
& y^{2}=\frac{3 \operatorname{sgn}\left(\Delta_{s}\right)}{2} \frac{\left[\Delta_{s}\left(\Delta_{2} \Delta_{6}-\Delta_{3} \Delta_{6}+\Delta_{4} \Delta_{5}\right)+\frac{1}{2}\left(\Delta_{5} \Delta_{8}-\Delta_{6} \Delta_{7}\right) \Delta_{7}\right]}{\sqrt{\left(\partial_{p} \Delta\right) \cdot(\mu \circ \alpha)\left(\partial_{p} \Delta\right)}} ; \\
& y^{3}= \frac{3 \operatorname{sgn}\left(\Delta_{s}\right)}{2} \frac{\left[\Delta_{s}\left(\Delta_{2} \Delta_{7}+\Delta_{3} \Delta_{7}+\Delta_{4} \Delta_{8}\right)+\frac{1}{2}\left(\Delta_{5} \Delta_{8}-\Delta_{6} \Delta_{7}\right) \Delta_{6}\right]}{\sqrt{\left(\partial_{p} \Delta\right) \cdot(\mu \circ \alpha)\left(\partial_{p} \Delta\right)}} ; \\
& y^{4}=\frac{3 \operatorname{sgn}\left(\Delta_{s}\right)}{2} \frac{\left[\Delta_{s}\left(\Delta_{2} \Delta_{8}-\Delta_{3} \Delta_{8}+\Delta_{4} \Delta_{7}\right)-\frac{1}{2}\left(\Delta_{5} \Delta_{8}-\Delta_{6} \Delta_{7}\right) \Delta_{5}\right]}{\sqrt{\left(\partial_{p} \Delta\right) \cdot(\mu \circ \alpha)\left(\partial_{p} \Delta\right)}} ;
\end{aligned}
$$


where

$$
\begin{aligned}
\left(\partial_{p} \Delta\right) \cdot(\mu \circ \alpha)\left(\partial_{p} \Delta\right)= & \Delta_{s}^{3}\left(-\Delta_{2}^{2}+\Delta_{3}^{2}+\Delta_{4}^{2}\right)+\frac{1}{4} \Delta_{s}\left(\Delta_{5} \Delta_{8}-\Delta_{6} \Delta_{7}\right)^{2} \\
& +\Delta_{s}^{2} \Delta_{2}\left[-2 \Delta_{s} \Delta_{2}+\frac{1}{2}\left(\Delta_{5}^{2}+\Delta_{6}^{2}+\Delta_{7}^{2}+\Delta_{8}^{2}\right)\right] \\
& +\Delta_{s}^{2} \Delta_{3}\left[2 \Delta_{s} \Delta_{3}+\frac{1}{2}\left(\Delta_{5}^{2}-\Delta_{6}^{2}+\Delta_{7}^{2}-\Delta_{8}^{2}\right)\right] \\
& +\Delta_{s}^{2} \Delta_{4}\left(2 \Delta_{s} \Delta_{4}+\Delta_{5} \Delta_{6}+\Delta_{7} \Delta_{8}\right) \\
& +\Delta_{s} \Delta_{5}\left[\Delta_{s}\left(\Delta_{2} \Delta_{5}+\Delta_{3} \Delta_{5}+\Delta_{4} \Delta_{6}\right)-\frac{1}{2}\left(\Delta_{5} \Delta_{8}-\Delta_{6} \Delta_{7}\right) \Delta_{8}\right] \\
& +\Delta_{s} \Delta_{6}\left[\Delta_{s}\left(\Delta_{2} \Delta_{6}-\Delta_{3} \Delta_{6}+\Delta_{4} \Delta_{5}\right)+\frac{1}{2}\left(\Delta_{5} \Delta_{8}-\Delta_{6} \Delta_{7}\right) \Delta_{7}\right] \\
& +\Delta_{s} \Delta_{7}\left[\Delta_{s}\left(\Delta_{2} \Delta_{7}+\Delta_{3} \Delta_{7}+\Delta_{4} \Delta_{8}\right)+\frac{1}{2}\left(\Delta_{5} \Delta_{8}-\Delta_{6} \Delta_{7}\right) \Delta_{6}\right] \\
& +\Delta_{s} \Delta_{8}\left[\Delta_{s}\left(\Delta_{2} \Delta_{8}-\Delta_{3} \Delta_{8}+\Delta_{4} \Delta_{7}\right)-\frac{1}{2}\left(\Delta_{5} \Delta_{8}-\Delta_{6} \Delta_{7}\right) \Delta_{5}\right] .
\end{aligned}
$$

Then, (6.66) and (6.73)-(6.75) respectively yield the corresponding full fledged expression of the BPS black hole entropy and of the BPS attractors:

$$
\begin{aligned}
\frac{S}{\pi} & =\frac{1}{3\left|p^{0}\right|} \sqrt{3 \frac{\left(\partial_{p} \Delta\right) \cdot(\mu \circ \alpha)\left(\partial_{p} \Delta\right)}{\Delta_{s}^{2}}-9\left[p^{0}(p \cdot q)-2 I_{3}(p)\right]^{2}} ; \\
z_{H}^{1}(\mathcal{Q}) & =\frac{3}{2} \frac{\left[\Delta_{s}^{2}\left(-\Delta_{2}^{2}+\Delta_{3}^{2}+\Delta_{4}^{2}\right)+\frac{1}{4}\left(\Delta_{5} \Delta_{8}-\Delta_{6} \Delta_{7}\right)^{2}\right]}{\left(\partial_{p} \Delta\right) \cdot(\mu \circ \alpha)\left(\partial_{p} \Delta\right)}\left[\frac{p^{0}(p \cdot q)-2 I_{3}(p)}{p^{0}}-\mathbf{i} \frac{3}{2} \frac{S}{\pi}\right]+\frac{p^{1}}{p^{0}} ; \\
z_{H}^{2}(\mathcal{Q}) & =\frac{3}{2} \frac{\Delta_{s}^{2}\left[-2 \Delta_{s} \Delta_{2}+\frac{1}{2}\left(\Delta_{5}^{2}+\Delta_{6}^{2}+\Delta_{7}^{2}+\Delta_{8}^{2}\right)\right]}{\left(\partial_{p} \Delta\right) \cdot(\mu \circ \alpha)\left(\partial_{p} \Delta\right)}\left[\frac{p^{0}(p \cdot q)-2 I_{3}(p)}{p^{0}}-\mathbf{i} \frac{3}{2} \frac{S}{\pi}\right]+\frac{p^{2}}{p^{0}} ; \\
z_{H}^{3}(\mathcal{Q}) & =\frac{3}{2} \frac{\Delta_{s}^{2}\left[2 \Delta_{s} \Delta_{3}+\frac{1}{2}\left(\Delta_{5}^{2}-\Delta_{6}^{2}+\Delta_{7}^{2}-\Delta_{8}^{2}\right)\right]}{\left(\partial_{p} \Delta\right) \cdot(\mu \circ \alpha)\left(\partial_{p} \Delta\right)}\left[\frac{p^{0}(p \cdot q)-2 I_{3}(p)}{p^{0}}-\mathbf{i} \frac{3}{2} \frac{S}{\pi}\right]+\frac{p^{3}}{p^{0}} ; \\
z_{H}^{4}(\mathcal{Q}) & =\frac{3}{2} \frac{\Delta_{s}^{2}\left(2 \Delta_{s} \Delta_{4}+\Delta_{5} \Delta_{6}+\Delta_{7} \Delta_{8}\right)}{\left(\partial_{p} \Delta\right) \cdot(\mu \circ \alpha)\left(\partial_{p} \Delta\right)}\left[\frac{p^{0}(p \cdot q)-2 I_{3}(p)}{p^{0}}-\mathbf{i} \frac{3}{2} \frac{S}{\pi}\right]+\frac{p^{4}}{p^{0}} ; \\
z_{H}^{5}(\mathcal{Q}) & =\frac{3}{2} \frac{\Delta_{s}\left[\Delta_{s}\left(\Delta_{2} \Delta_{5}+\Delta_{3} \Delta_{5}+\Delta_{4} \Delta_{6}\right)-\frac{1}{2}\left(\Delta_{5} \Delta_{8}-\Delta_{6} \Delta_{7}\right) \Delta_{8}\right]}{\left(\partial_{p} \Delta\right) \cdot(\mu \circ \alpha)\left(\partial_{p} \Delta\right)}\left[\frac{p^{0}(p \cdot q)-2 I_{3}(p)}{p^{0}}-\mathbf{i} \frac{3}{2} \frac{S}{\pi}\right]+\frac{p^{5}}{p^{0}} \\
z_{H}^{6}(\mathcal{Q}) & =\frac{3}{2} \frac{\Delta_{s}\left[\Delta_{s}\left(\Delta_{2} \Delta_{6}-\Delta_{3} \Delta_{6}+\Delta_{4} \Delta_{5}\right)+\frac{1}{2}\left(\Delta_{5} \Delta_{8}-\Delta_{6} \Delta_{7}\right) \Delta_{7}\right]}{\left(\partial_{p} \Delta\right) \cdot(\mu \circ \alpha)\left(\partial_{p} \Delta\right)}\left[\frac{p^{0}(p \cdot q)-2 I_{3}(p)}{p^{0}}-\mathbf{i} \frac{3}{2} \frac{S}{\pi}\right]+\frac{p^{6}}{p^{0}} ; \\
z_{H}^{7}(\mathcal{Q}) & =\frac{3}{2} \frac{\Delta_{s}\left[\Delta_{s}\left(\Delta_{2} \Delta_{7}+\Delta_{3} \Delta_{7}+\Delta_{4} \Delta_{8}\right)+\frac{1}{2}\left(\Delta_{5} \Delta_{8}-\Delta_{6} \Delta_{7}\right) \Delta_{6}\right]}{\left(\partial_{p} \Delta\right) \cdot(\mu \circ \alpha)\left(\partial_{p} \Delta\right)}\left[\frac{p^{0}(p \cdot q)-2 I_{3}(p)}{p^{0}}-\mathbf{i} \frac{3}{2} \frac{S}{\pi}\right]+\frac{p^{7}}{p^{0}} \\
z_{H}^{8}(\mathcal{Q}) & =\frac{3}{2} \frac{\Delta_{s}\left[\Delta_{s}\left(\Delta_{2} \Delta_{8}-\Delta_{3} \Delta_{8}+\Delta_{4} \Delta_{7}\right)-\frac{1}{2}\left(\Delta_{5} \Delta_{8}-\Delta_{6} \Delta_{7}\right) \Delta_{5}\right]}{\left(\partial_{p} \Delta\right) \cdot(\mu \circ \alpha)\left(\partial_{p} \Delta\right)}\left[\frac{p^{0}(p \cdot q)-2 I_{3}(p)}{p^{0}}-\mathbf{i} \frac{3}{2} \frac{S}{\pi}\right]+\frac{p^{8}}{p^{0}}
\end{aligned}
$$


with $\left(\partial_{p} \Delta\right) \cdot(\mu \circ \alpha)\left(\partial_{p} \Delta\right)$ given by (A.22), and

$$
\begin{aligned}
p \cdot q= & p^{0} q_{0}+p^{1} q_{1}+\ldots+p^{8} q_{8} \\
I_{3}(p)= & \mathcal{V}_{L(1,2)}\left(p^{1}, \ldots, p^{8}\right)=p^{1}\left[-\left(p^{2}\right)^{2}+\left(p^{3}\right)^{2}+\left(p^{4}\right)^{2}\right] \\
& +p^{2} Q_{0}^{(2)}\left(p^{5}, \ldots, p^{8}\right)+p^{3} Q_{1}^{(2)}\left(p^{5}, \ldots, p^{8}\right)+p^{4} Q_{2}^{(2)}\left(p^{5}, \ldots, p^{8}\right) \\
= & p^{1}\left[-\left(p^{2}\right)^{2}+\left(p^{3}\right)^{2}+\left(p^{4}\right)^{2}\right]+p^{2}\left[\left(p^{5}\right)^{2}+\left(p^{6}\right)^{2}+\left(p^{7}\right)^{2}+\left(p^{8}\right)^{2}\right] \\
& +p^{3}\left[\left(p^{5}\right)^{2}-\left(p^{6}\right)^{2}+\left(p^{7}\right)^{2}-\left(p^{8}\right)^{2}\right]+2 p^{4}\left(p^{5} p^{6}+p^{7} p^{8}\right) .
\end{aligned}
$$

\section{B Details on $L(1,3)$}

The cubic form $\mathcal{V}_{L(1,3)}$ reads

$$
\mathcal{V}_{L(1,3)}=s q(x)+\sum_{I=0}^{2} x^{I} Q_{I}^{(3)}(y), \quad \text { with } \quad q(x)=-\left(x^{0}\right)^{2}+\left(x^{1}\right)^{2}+\left(x^{2}\right)^{2}
$$

where the $Q_{I}^{(3)}$ are obtained from the $Q_{I}$ 's of the $L(1,1)$ model (cfr. (8.70)):

$$
\begin{aligned}
& Q_{0}^{(3)}=\left(y^{1}\right)^{2}+\left(y^{2}\right)^{2}+\left(y^{3}\right)^{2}+\left(y^{4}\right)^{2}+\left(y^{5}\right)^{2}+\left(y^{6}\right)^{2} \\
& Q_{1}^{(3)}=\left(y^{1}\right)^{2}-\left(y^{2}\right)^{2}+\left(y^{3}\right)^{2}-\left(y^{4}\right)^{2}+\left(y^{5}\right)^{2}-\left(y^{6}\right)^{2} \\
& Q_{2}^{(3)}=2\left(y^{1} y^{2}+y^{3} y^{4}+y^{5} y^{6}\right) .
\end{aligned}
$$

Moreover, we define the quadrics, based on the quadric $R$ of the $L(1,2)$ model (see (A.3)), $R^{(k, l)}(y):=R\left(y^{(k l)}\right)$ :

$$
R^{(1,2)}=2\left(y^{1} y^{4}-y^{2} y^{3}\right), \quad R^{(1,3)}=2\left(y^{1} y^{6}-y^{2} y^{5}\right), \quad R^{(2,3)}=2\left(y^{3} y^{6}-y^{4} y^{5}\right) .
$$

As required by the invertibility condition of section 6.2 (cfr. (6.8)), a Lorentzian identity of type (10.4) holds true, namely,

$$
-Q_{0}^{(3)}(y)^{2}+Q_{1}^{(3)}(y)^{2}+Q_{2}^{(3)}(y)^{2}+R^{(1,2)}(y)^{2}+R^{(1,3)}(y)^{2}+R^{(2,3)}(y)^{2}=0 .
$$

Since $r=3$, the inverse of the gradient map $\nabla \mathcal{V}_{L(1,3)}$ is given as a composition of two maps, namely (cfr. (4.41))

$$
\mathbb{R}_{\xi}^{1+3+6} \stackrel{\nabla_{\mathcal{V}_{L(1,3)}}^{\longrightarrow}}{\longrightarrow} \mathbb{R}_{z}^{1+3+6} \stackrel{\alpha}{\longrightarrow} \mathbb{R}_{(t, u, v, w)}^{1+3+6+3} \stackrel{\mu}{\longrightarrow} \mathbb{R}_{\xi}^{1+3+6},
$$

with

$$
\mu \circ \alpha \circ \nabla_{\mathcal{V}_{L(1,3)}}(\xi)=4 q(x)^{2} \mathcal{V}_{L(1,3)}(\xi) \xi,
$$

where $\xi=(s, x, y)$, and $\mathcal{V}_{L(1,3)}$ and the corresponding $q(x)$ are given by (B.1). The map $\alpha$ from (6.14) is given by

$$
\alpha\left(z_{1}, \ldots, z_{10}\right):={ }^{T}\left(z_{1}^{2}, z_{1} z_{2}, \ldots, z_{1} z_{10}, R^{(1,2)}(z), R^{(1,3)}(z), R^{(2,3)}(z)\right),
$$


where each of the three quadratic forms $R^{(k, l)}(z)$ 's depends only on four of the last $3 \cdot 2=6$ variables:

$$
R^{(1,2)}(z)=R\left(z_{5}, \ldots, z_{8}\right), \quad R^{(1,3)}(z)=R\left(z_{5}, z_{6}, z_{9}, z_{10}\right), \quad R^{(2,3)}(z)=R\left(z_{7}, \ldots, z_{10}\right)
$$

The map $\mu$ (6.17) has $1+3+6$ components which are homogeneous polynomials of degree 2 in the variables $t, u_{0}, u_{1}, u_{2}, v_{1}, \ldots, v_{6}, w_{1}, w_{2}, w_{3}$, and it is given by

$$
\mu(t, u, v, w):=\left(\begin{array}{c}
\mu: \mathbb{R}_{t, u, v, w}^{1+3+6+3} \longrightarrow \mathbb{R}_{\xi}^{1+3+6} \\
-u_{0}^{2}+u_{1}^{2}+u_{2}^{2}+\frac{1}{16}\left(w_{1}^{2}+w_{2}^{2}+w_{3}^{2}\right) \\
-2 t u_{0}+\frac{1}{2}\left(v_{1}^{2}+v_{2}^{2}+v_{3}^{2}+v_{4}^{2}+v_{5}^{2}+v_{6}^{2}\right) \\
2 t u_{1}+\frac{1}{16}\left(v_{1}^{2}-v_{2}^{2}+v_{3}^{2}-v_{4}^{2}+v_{5}^{2}-v_{6}^{2}\right) \\
2 t u_{2}+v_{1} v_{2}+v_{3} v_{4}+v_{5} v_{6} \\
u_{0} v_{1}+u_{1} v_{1}+u_{2} v_{2}+\frac{1}{4}\left(-v_{4} w_{1}-v_{6} w_{2}\right) \\
u_{0} v_{2}-u_{1} v_{2}+u_{2} v_{1}+\frac{1}{4}\left(v_{3} w_{1}+v_{5} w_{2}\right) \\
u_{0} v_{3}+u_{1} v_{3}+u_{2} v_{4}+\frac{1}{4}\left(v_{2} w_{1}-v_{6} w_{3}\right) \\
u_{0} v_{4}-u_{1} v_{4}+u_{2} v_{3}+\frac{1}{4}\left(-v_{1} w_{1}-v_{5} w_{3}\right) \\
u_{0} v_{5}+u_{1} v_{5}+u_{2} v_{6}+\frac{1}{4}\left(v_{2} w_{2}+v_{4} w_{3}\right) \\
u_{0} v_{6}-u_{1} v_{6}+u_{2} v_{5}+\frac{1}{4}\left(-v_{1} w_{2}-v_{3} w_{3}\right)
\end{array}\right) .
$$

Therefore, the (birational) inverse map $\left(\nabla_{\mathcal{V}_{L(1,3)}}\right)^{-1}=\mu \circ \alpha$ of the gradient map $\nabla_{\mathcal{V}_{L(1,3)}}$ reads (cfr. (6.27))

$$
\begin{gathered}
\mu \circ \alpha: \mathbb{R}_{z}^{1+3+6} \stackrel{\alpha}{\longrightarrow} \mathbb{R}_{(t, u, v, w)}^{1+3+6+3} \stackrel{\mu}{\longrightarrow} \mathbb{R}_{\xi}^{1+3+6} \\
(\mu \circ \alpha)(z):=\left(\begin{array}{l}
z_{1}^{2}\left(-z_{2}^{2}+z_{3}^{2}+z_{4}^{2}\right)+\frac{1}{4}\left[\left(z_{5} z_{8}-z_{6} z_{7}\right)^{2}+\left(z_{7} z_{10}-z_{8} z_{9}\right)^{2}\right] \\
z_{1}^{2}\left[-2 z_{1} z_{2}+\frac{1}{2}\left(z_{5}^{2}+z_{6}^{2}+z_{7}^{2}+z_{8}^{2}+z_{9}^{2}+z_{10}^{2}\right)\right] \\
z_{1}^{2}\left[2 z_{1} z_{3}+\frac{1}{2}\left(z_{5}^{2}-z_{6}^{2}+z_{7}^{2}-z_{8}^{2}+z_{9}^{2}-z_{10}^{2}\right)\right] \\
z_{1}^{2}\left(2 z_{1} z_{4}+z_{5} z_{6}+z_{7} z_{8}+z_{9} z_{10}\right) \\
z_{1}^{2}\left(z_{2} z_{5}+z_{3} z_{5}+z_{4} z_{6}\right)+\frac{1}{2} z_{1}\left(-z_{5} z_{8}^{2}-z_{5} z_{10}^{2}+z_{6} z_{7} z_{8}+z_{6} z_{9} z_{10}\right) \\
z_{1}^{2}\left(z_{2} z_{6}-z_{3} z_{6}+z_{4} z_{5}\right)+\frac{1}{2} z_{1}\left(z_{5} z_{7} z_{8}+z_{5} z_{9} z_{10}-z_{6} z_{7}^{2}-z_{6} z_{9}^{2}\right) \\
z_{1}^{2}\left(z_{2} z_{7}+z_{3} z_{7}+z_{4} z_{8}\right)+\frac{1}{2} z_{1}\left(z_{5} z_{6} z_{8}-z_{6}^{2} z_{7}-z_{7} z_{10}^{2}+z_{8} z_{9} z_{10}\right) \\
z_{1}^{2}\left(z_{2} z_{8}-z_{3} z_{8}+z_{4} z_{7}\right)+\frac{1}{2} z_{1}\left(-z_{5}^{2} z_{8}+z_{5} z_{6} z_{7}+z_{7} z_{9} z_{10}-z_{8} z_{9}^{2}\right) \\
z_{1}^{2}\left(z_{2} z_{9}+z_{3} z_{9}+z_{4} z_{10}\right)+\frac{1}{2} z_{1}\left(z_{5} z_{6} z_{10}-z_{6}^{2} z_{9}+z_{7} z_{8} z_{10}-z_{8}^{2} z_{9}\right) \\
z_{1}^{2}\left(z_{2} z_{10}-z_{3} z_{10}+z_{4} z_{9}\right)+\frac{1}{2} z_{1}\left(-z_{5}^{2} z_{10}+z_{5} z_{6} z_{9}-z_{7}^{2} z_{10}+z_{7} z_{8} z_{9}\right)
\end{array}\right) .
\end{gathered}
$$


Consequently, from the treatment of section 6, the full fledged expression of the solution (6.53) of the BPS system of $L(1,3)$ is given by $\left(\Delta_{1} \equiv \Delta_{s}\right.$, and recall the definition (2.3)):

$$
\begin{aligned}
s & =\frac{3}{2\left|\Delta_{s}\right|} \frac{\left\{\Delta_{s}^{2}\left(-\Delta_{2}^{2}+\Delta_{3}^{2}+\Delta_{4}^{2}\right)+\frac{1}{4}\left[\left(\Delta_{5} \Delta_{8}-\Delta_{6} \Delta_{7}\right)^{2}+\left(\Delta_{7} \Delta_{10}-\Delta_{8} \Delta_{9}\right)^{2}\right]\right\}}{\sqrt{\left(\partial_{p} \Delta\right) \cdot(\mu \circ \alpha)\left(\partial_{p} \Delta\right)}} ; \\
x^{0} & =\frac{3}{2} \frac{\left|\Delta_{s}\right|\left[-2 \Delta_{s} \Delta_{2}+\frac{1}{2}\left(\Delta_{5}^{2}+\Delta_{6}^{2}+\Delta_{7}^{2}+\Delta_{8}^{2}+\Delta_{9}^{2}+\Delta_{10}^{2}\right)\right]}{\sqrt{\left(\partial_{p} \Delta\right) \cdot(\mu \circ \alpha)\left(\partial_{p} \Delta\right)}} ; \\
x^{1} & =\frac{3}{2} \frac{\left|\Delta_{s}\right|\left[2 \Delta_{s} \Delta_{3}+\frac{1}{2}\left(\Delta_{5}^{2}-\Delta_{6}^{2}+\Delta_{7}^{2}-\Delta_{8}^{2}+\Delta_{9}^{2}-\Delta_{10}^{2}\right)\right]}{\sqrt{\left(\partial_{p} \Delta\right) \cdot(\mu \circ \alpha)\left(\partial_{p} \Delta\right)}} ; \\
x^{2} & =\frac{3}{2} \frac{\left|\Delta_{s}\right|\left(2 \Delta_{s} \Delta_{4}+\Delta_{5} \Delta_{6}+\Delta_{7} \Delta_{8}+\Delta z_{9} \Delta_{10}\right)}{\sqrt{\left(\partial_{p} \Delta\right) \cdot(\mu \circ \alpha)\left(\partial_{p} \Delta\right)}} ; \\
y^{1} & =\frac{3}{2} \frac{\left[\left|\Delta_{s}\right|\left(\Delta_{2} \Delta_{5}+\Delta_{3} \Delta_{5}+\Delta_{4} \Delta_{6}\right)+\frac{1}{2} \operatorname{sgn}\left(\Delta_{s}\right)\left(-\Delta_{5} \Delta_{8}^{2}-\Delta_{5} \Delta_{10}^{2}+\Delta_{6} \Delta_{7} \Delta_{8}+\Delta_{6} \Delta_{9} \Delta_{10}\right)\right]}{\sqrt{\left(\partial_{p} \Delta\right) \cdot(\mu \circ \alpha)\left(\partial_{p} \Delta\right)}} ; \\
y^{2} & =\frac{3}{2} \frac{\left[\left|\Delta_{s}\right|\left(\Delta_{2} \Delta_{6}-\Delta_{3} \Delta_{6}+\Delta_{4} \Delta_{5}\right)+\frac{1}{2} \operatorname{sgn}\left(\Delta_{s}\right)\left(\Delta_{5} \Delta_{7} \Delta_{8}+\Delta_{5} \Delta_{9} \Delta_{10}-\Delta_{6} \Delta_{7}^{2}-\Delta_{6} \Delta_{9}^{2}\right)\right]}{\sqrt{\left(\partial_{p} \Delta\right) \cdot(\mu \circ \alpha)\left(\partial_{p} \Delta\right)}} ; \\
y^{3} & =\frac{3}{2} \frac{\left[\left|\Delta_{s}\right|\left(\Delta_{2} \Delta_{7}+\Delta_{3} \Delta_{+}+\Delta_{4} \Delta_{8}\right)+\frac{1}{2} \operatorname{sgn}\left(\Delta_{s}\right)\left(\Delta_{5} \Delta_{6} \Delta_{8}-\Delta_{6}^{2} \Delta_{7}-\Delta_{7} \Delta_{10}^{2}+\Delta_{8} \Delta_{9} \Delta_{10}\right)\right]}{\sqrt{\left(\partial_{p} \Delta\right) \cdot(\mu \circ \alpha)\left(\partial_{p} \Delta\right)}} ; \\
y^{4} & =\frac{3}{2} \frac{\left[\left|\Delta_{s}\right|\left(\Delta_{2} \Delta_{8}-\Delta_{3} \Delta_{8}+\Delta_{4} \Delta_{7}\right)+\frac{1}{2} \operatorname{sgn}\left(\Delta_{s}\right)\left(-\Delta_{5}^{2} \Delta_{8}+\Delta_{5} \Delta_{6} \Delta_{7}+\Delta_{7} \Delta_{9} \Delta_{10}-\Delta_{8} \Delta_{9}^{2}\right)\right]}{\sqrt{\left(\partial_{p} \Delta\right) \cdot(\mu \circ \alpha)\left(\partial_{p} \Delta\right)}} ; \\
y^{5} & =\frac{3}{2} \frac{\left[\left|\Delta_{s}\right|\left(\Delta_{2} \Delta_{9}+\Delta_{3} \Delta_{9}+\Delta_{4} \Delta_{10}\right)+\frac{1}{2} \operatorname{sgn}\left(\Delta_{s}\right)\left(\Delta_{5} \Delta_{6} \Delta_{10}-\Delta_{6}^{2} \Delta_{9}+\Delta_{7} \Delta_{8} \Delta_{10}-\Delta_{8}^{2} \Delta_{9}\right)\right]}{\sqrt{\left(\partial_{p} \Delta\right) \cdot(\mu \circ \alpha)\left(\partial_{p} \Delta\right)}} ; \\
y^{6} & =\frac{3}{2} \frac{\left[\left|\Delta_{s}\right|\left(\Delta_{2} \Delta_{10}-\Delta_{3} \Delta_{10}+\Delta_{4} \Delta_{9}\right)+\frac{1}{2} \operatorname{sgn}\left(\Delta_{s}\right)\left(-\Delta_{5}^{2} \Delta_{10}+\Delta_{5} \Delta_{6} \Delta_{9}-\Delta_{7}^{2} \Delta_{10}+\Delta_{7} \Delta_{8} \Delta_{9}\right)\right]}{\sqrt{\left(\partial_{p} \Delta\right) \cdot(\mu \circ \alpha)\left(\partial_{p} \Delta\right)}},
\end{aligned}
$$

where

$$
\begin{aligned}
\left(\partial_{p} \Delta\right) \cdot(\mu \circ \alpha)\left(\partial_{p} \Delta\right)= & \Delta_{s}^{3}\left(-\Delta_{2}^{2}+\Delta_{3}^{2}+\Delta_{4}^{2}\right) \\
& +\frac{1}{4} \Delta_{s}\left[\left(\Delta_{5} \Delta_{8}-\Delta_{6} \Delta_{7}\right)^{2}+\left(\Delta_{7} \Delta_{10}-\Delta_{8} \Delta_{9}\right)^{2}\right] \\
& +\Delta_{s}^{2} \Delta_{2}\left[-2 \Delta_{s} \Delta_{2}+\frac{1}{2}\left(\Delta_{5}^{2}+\Delta_{6}^{2}+\Delta_{7}^{2}+\Delta_{8}^{2}+\Delta_{9}^{2}+\Delta_{10}^{2}\right)\right] \\
& +\Delta_{s}^{2} \Delta_{3}\left[2 \Delta_{s} \Delta_{3}+\frac{1}{2}\left(\Delta_{5}^{2}-\Delta_{6}^{2}+\Delta_{7}^{2}-\Delta_{8}^{2}+\Delta_{9}^{2}-\Delta_{10}^{2}\right)\right] \\
& +\Delta_{s}^{2} \Delta_{4}\left(2 \Delta_{s} \Delta_{4}+\Delta_{5} \Delta_{6}+\Delta_{7} \Delta_{8}+\Delta_{9} \Delta_{10}\right) \\
& +\Delta_{s}^{2} \Delta_{5}\left(\Delta_{2} \Delta_{5}+\Delta_{3} \Delta_{5}+\Delta_{4} \Delta_{6}\right) \\
& +\frac{1}{2} \Delta_{s} \Delta_{5}\left(-\Delta_{5} \Delta_{8}^{2}-\Delta_{5} \Delta_{10}^{2}+\Delta_{6} \Delta_{7} \Delta_{8}+\Delta_{6} \Delta_{9} \Delta_{10}\right) \\
& +\Delta_{s}^{2} \Delta_{6}\left(\Delta_{2} \Delta_{6}-\Delta_{3} \Delta_{6}+\Delta_{4} \Delta_{5}\right) \\
& +\frac{1}{2} \Delta_{s} \Delta_{6}\left(\Delta_{5} \Delta_{7} \Delta_{8}+\Delta_{5} \Delta_{9} \Delta_{10}-\Delta_{6} \Delta_{7}^{2}-\Delta_{6} \Delta_{9}^{2}\right) \\
& +\Delta_{s}^{2} \Delta_{7}\left(\Delta_{2} \Delta_{7}+\Delta_{3} \Delta_{4} \Delta_{4} \Delta_{8}\right) \\
& +\frac{1}{2} \Delta_{s} \Delta_{7}\left(\Delta_{5} \Delta_{6} \Delta_{8}-\Delta_{6}^{2} \Delta_{7}-\Delta_{7} \Delta_{10}^{2}+\Delta_{8} \Delta_{9} \Delta_{10}\right) \\
& +\Delta_{s}^{2} \Delta_{8}\left(\Delta_{2} \Delta_{8}-\Delta_{3} \Delta_{8}+\Delta_{4} \Delta_{7}\right)
\end{aligned}
$$




$$
\begin{aligned}
& +\frac{1}{2} \Delta_{s} \Delta_{8}\left(-\Delta_{5}^{2} \Delta_{8}+\Delta_{5} \Delta_{6} \Delta_{7}+\Delta_{7} \Delta_{9} \Delta_{10}-\Delta_{8} \Delta_{9}^{2}\right) \\
& +\Delta_{s}^{2} \Delta_{9}\left(\Delta_{2} \Delta_{9}+\Delta_{3} \Delta_{9}+\Delta_{4} \Delta_{10}\right) \\
& +\frac{1}{2} \Delta_{s} \Delta_{9}\left(\Delta_{5} \Delta_{6} \Delta_{10}-\Delta_{6}^{2} \Delta_{9}+\Delta_{7} \Delta_{8} \Delta_{10}-\Delta_{8}^{2} \Delta_{9}\right) \\
& +\Delta_{s}^{2} \Delta_{10}\left(\Delta_{2} \Delta_{10}-\Delta_{3} \Delta_{10}+\Delta_{4} \Delta_{9}\right) \\
& +\frac{1}{2} \Delta_{s} \Delta_{10}\left(-\Delta_{5}^{2} \Delta_{10}+\Delta_{5} \Delta_{6} \Delta_{9}-\Delta_{7}^{2} \Delta_{10}+\Delta_{7} \Delta_{8} \Delta_{9}\right)
\end{aligned}
$$

Then, (6.66) and (6.73)-(6.75) respectively yield the corresponding full fledged expression of the BPS black hole entropy and of the BPS attractors:

$$
\begin{aligned}
& \frac{S}{\pi}=\frac{1}{3\left|p^{0}\right|} \sqrt{3 \frac{\left(\partial_{p} \Delta\right) \cdot(\mu \circ \alpha)\left(\partial_{p} \Delta\right)}{\Delta_{s}^{2}}-9\left[p^{0}(p \cdot q)-2 I_{3}(p)\right]^{2}} ; \\
& z_{H}^{1}(\mathcal{Q})=\frac{3}{2} \frac{\left\{\Delta_{s}^{2}\left(-\Delta_{2}^{2}+\Delta_{3}^{2}+\Delta_{4}^{2}\right)+\frac{1}{4}\left[\left(\Delta_{5} \Delta_{8}-\Delta_{6} \Delta_{7}\right)^{2}+\left(\Delta_{7} \Delta_{10}-\Delta_{8} \Delta_{9}\right)^{2}\right]\right\}}{\left(\partial_{p} \Delta\right) \cdot(\mu \circ \alpha)\left(\partial_{p} \Delta\right)} . \\
& \cdot\left[\frac{p^{0}(p \cdot q)-2 I_{3}(p)}{p^{0}}-\mathbf{i} \frac{3}{2} \frac{S}{\pi}\right]+\frac{p^{1}}{p^{0}} ; \\
& z_{H}^{2}(\mathcal{Q})=\frac{3}{2} \frac{\Delta_{s}^{2}\left[-2 \Delta_{s} \Delta_{2}+\frac{1}{2}\left(\Delta_{5}^{2}+\Delta_{6}^{2}+\Delta_{7}^{2}+\Delta_{8}^{2}+\Delta_{9}^{2}+\Delta_{10}^{2}\right)\right]}{\left(\partial_{p} \Delta\right) \cdot(\mu \circ \alpha)\left(\partial_{p} \Delta\right)}\left[\frac{p^{0}(p \cdot q)-2 I_{3}(p)}{p^{0}}-\mathbf{i} \frac{3}{2} \frac{S}{\pi}\right]+\frac{p^{2}}{p^{0}} ; \\
& z_{H}^{3}(\mathcal{Q})=\frac{3}{2} \frac{\Delta_{s}^{2}\left[2 \Delta_{s} \Delta_{3}+\frac{1}{2}\left(\Delta_{5}^{2}-\Delta_{6}^{2}+\Delta_{7}^{2}-\Delta_{8}^{2}+\Delta_{9}^{2}-\Delta_{10}^{2}\right)\right]}{\left(\partial_{p} \Delta\right) \cdot(\mu \circ \alpha)\left(\partial_{p} \Delta\right)}\left[\frac{p^{0}(p \cdot q)-2 I_{3}(p)}{p^{0}}-\mathbf{i} \frac{3}{2} \frac{S}{\pi}\right]+\frac{p^{3}}{p^{0}} ; \\
& z_{H}^{4}(\mathcal{Q})=\frac{3}{2} \frac{\Delta_{s}^{2}\left(2 \Delta_{s} \Delta_{4}+\Delta_{5} \Delta_{6}+\Delta_{7} \Delta_{8}+\Delta z_{9} \Delta_{10}\right)}{\left(\partial_{p} \Delta\right) \cdot(\mu \circ \alpha)\left(\partial_{p} \Delta\right)}\left[\frac{p^{0}(p \cdot q)-2 I_{3}(p)}{p^{0}}-\mathbf{i} \frac{3}{2} \frac{S}{\pi}\right]+\frac{p^{4}}{p^{0}} \\
& z_{H}^{5}(\mathcal{Q})=\frac{3}{2} \frac{\left[\Delta_{s}^{2}\left(\Delta_{2} \Delta_{5}+\Delta_{3} \Delta_{5}+\Delta_{4} \Delta_{6}\right)+\frac{1}{2} \Delta_{s}\left(-\Delta_{5} \Delta_{8}^{2}-\Delta_{5} \Delta_{10}^{2}+\Delta_{6} \Delta_{7} \Delta_{8}+\Delta_{6} \Delta_{9} \Delta_{10}\right)\right]}{\left(\partial_{p} \Delta\right) \cdot(\mu \circ \alpha)\left(\partial_{p} \Delta\right)} . \\
& \cdot\left[\frac{p^{0}(p \cdot q)-2 I_{3}(p)}{p^{0}}-\mathbf{i} \frac{3}{2} \frac{S}{\pi}\right]+\frac{p^{5}}{p^{0}} \\
& z_{H}^{6}(\mathcal{Q})=\frac{3}{2} \frac{\left[\Delta_{s}^{2}\left(\Delta_{2} \Delta_{6}-\Delta_{3} \Delta_{6}+\Delta_{4} \Delta_{5}\right)+\frac{1}{2} \Delta_{s}\left(\Delta_{5} \Delta_{7} \Delta_{8}+\Delta_{5} \Delta_{9} \Delta_{10}-\Delta_{6} \Delta_{7}^{2}-\Delta_{6} \Delta_{9}^{2}\right)\right]}{\left(\partial_{p} \Delta\right) \cdot(\mu \circ \alpha)\left(\partial_{p} \Delta\right)} . \\
& \cdot\left[\frac{p^{0}(p \cdot q)-2 I_{3}(p)}{p^{0}}-\mathbf{i} \frac{3}{2} \frac{S}{\pi}\right]+\frac{p^{6}}{p^{0}} \\
& z_{H}^{7}(\mathcal{Q})=\frac{3}{2} \frac{\left[\Delta_{s}^{2}\left(\Delta_{2} \Delta_{7}+\Delta_{3} \Delta+\Delta_{4} \Delta_{8}\right)+\frac{1}{2} \Delta_{s}\left(\Delta_{5} \Delta_{6} \Delta_{8}-\Delta_{6}^{2} \Delta_{7}-\Delta_{7} \Delta_{10}^{2}+\Delta_{8} \Delta_{9} \Delta_{10}\right)\right]}{\left(\partial_{p} \Delta\right) \cdot(\mu \circ \alpha)\left(\partial_{p} \Delta\right)} . \\
& \cdot\left[\frac{p^{0}(p \cdot q)-2 I_{3}(p)}{p^{0}}-\mathbf{i} \frac{3}{2} \frac{S}{\pi}\right]+\frac{p^{7}}{p^{0}} ; \\
& z_{H}^{8}(\mathcal{Q})=\frac{3}{2} \frac{\left[\Delta_{s}^{2}\left(\Delta_{2} \Delta_{8}-\Delta_{3} \Delta_{8}+\Delta_{4} \Delta_{7}\right)+\frac{1}{2} \Delta_{s}\left(-\Delta_{5}^{2} \Delta_{8}+\Delta_{5} \Delta_{6} \Delta_{7}+\Delta_{7} \Delta_{9} \Delta_{10}-\Delta_{8} \Delta_{9}^{2}\right)\right]}{\left(\partial_{p} \Delta\right) \cdot(\mu \circ \alpha)\left(\partial_{p} \Delta\right)} . \\
& \cdot\left[\frac{p^{0}(p \cdot q)-2 I_{3}(p)}{p^{0}}-\mathbf{i} \frac{3}{2} \frac{S}{\pi}\right]+\frac{p^{8}}{p^{0}} ; \\
& z_{H}^{9}(\mathcal{Q})=\frac{3}{2} \frac{\left[\Delta_{s}^{2}\left(\Delta_{2} \Delta_{9}+\Delta_{3} \Delta_{9}+\Delta_{4} \Delta_{10}\right)+\frac{1}{2} \Delta_{s}\left(\Delta_{5} \Delta_{6} \Delta_{10}-\Delta_{6}^{2} \Delta_{9}+\Delta_{7} \Delta_{8} \Delta_{10}-\Delta_{8}^{2} \Delta_{9}\right)\right]}{\left(\partial_{p} \Delta\right) \cdot(\mu \circ \alpha)\left(\partial_{p} \Delta\right)} . \\
& \cdot\left[\frac{p^{0}(p \cdot q)-2 I_{3}(p)}{p^{0}}-\mathbf{i} \frac{3}{2} \frac{S}{\pi}\right]+\frac{p^{9}}{p^{0}} ;
\end{aligned}
$$




$$
\begin{aligned}
z_{H}^{10}(\mathcal{Q})= & \frac{3}{2} \frac{\left[\Delta_{s}^{2}\left(\Delta_{2} \Delta_{10}-\Delta_{3} \Delta_{10}+\Delta_{4} \Delta_{9}\right)+\frac{1}{2} \Delta_{s}\left(-\Delta_{5}^{2} \Delta_{10}+\Delta_{5} \Delta_{6} \Delta_{9}-\Delta_{7}^{2} \Delta_{10}+\Delta_{7} \Delta_{8} \Delta_{9}\right)\right]}{\left(\partial_{p} \Delta\right) \cdot(\mu \circ \alpha)\left(\partial_{p} \Delta\right)} \\
& \cdot\left[\frac{p^{0}(p \cdot q)-2 I_{3}(p)}{p^{0}}-\mathbf{i} \frac{3}{2} \frac{S}{\pi}\right]+\frac{p^{10}}{p^{0}}
\end{aligned}
$$

with $\left(\partial_{p} \Delta\right) \cdot(\mu \circ \alpha)\left(\partial_{p} \Delta\right)$ given by (B.25), and

$$
\begin{aligned}
p \cdot q= & p^{0} q_{0}+p^{1} q_{1}+\ldots+p^{10} q_{10} ; \\
I_{3}(p)= & \mathcal{V}_{L(1,3)}\left(p^{1}, \ldots, p^{10}\right)=p^{1}\left[-\left(p^{2}\right)^{2}+\left(p^{3}\right)^{2}+\left(p^{4}\right)^{2}\right]+p^{2} Q_{0}^{(3)}\left(p^{5}, \ldots, p^{10}\right) \\
& +p^{3} Q_{1}^{(3)}\left(p^{5}, \ldots, p^{10}\right)+p^{4} Q_{2}^{(2)}\left(p^{5}, \ldots, p^{10}\right) \\
= & p^{1}\left[-\left(p^{2}\right)^{2}+\left(p^{3}\right)^{2}+\left(p^{4}\right)^{2}\right]+p^{2}\left[\left(p^{5}\right)^{2}+\left(p^{6}\right)^{2}+\left(p^{7}\right)^{2}+\left(p^{8}\right)^{2}+\left(p^{9}\right)^{2}+\left(p^{10}\right)^{2}\right] \\
& +p^{3}\left[\left(p^{5}\right)^{2}-\left(p^{6}\right)^{2}+\left(p^{7}\right)^{2}-\left(p^{8}\right)^{2}+\left(p^{9}\right)^{2}-\left(p^{10}\right)^{2}\right]+2 p^{4}\left(p^{5} p^{6}+p^{7} p^{8}+p^{9} p^{10}\right) .
\end{aligned}
$$

\section{A calculation in Lie theory}

With no loss of generality, one can consider Lie algebras on $\mathbb{C}$, and classify all inequivalent (up to $\mathfrak{d}_{5}$ inner automorphisms) algebras $\mathfrak{a}_{1} \oplus \mathfrak{b}_{2}$ in $\mathfrak{d}_{5}$, then considering also the branching of the $\mathbf{1 0} \oplus \mathbf{1 6}$ of $\mathfrak{d}_{5}$ in irreprs. of $\mathfrak{a}_{1} \oplus \mathfrak{b}_{2}$ :

I: $\mathfrak{d}_{5} \rightarrow \mathfrak{a}_{1, I} \oplus \mathfrak{a}_{1, I I} \oplus \mathfrak{a}_{3} \rightarrow \mathfrak{a}_{1, I} \oplus \mathfrak{a}_{1, I I} \oplus \mathfrak{b}_{2}$

$$
\begin{aligned}
& \text { symmetric in } I, I I \\
& =(\mathbf{3}, \mathbf{1}, \mathbf{1}) \oplus(\mathbf{1}, \mathbf{3}, \mathbf{1}) \oplus(\mathbf{1}, \mathbf{1}, \mathbf{1 5}) \oplus(\mathbf{2}, \mathbf{2}, \mathbf{6}) \\
& =(\mathbf{3}, \mathbf{1}, \mathbf{1}) \oplus(\mathbf{1}, \mathbf{3}, \mathbf{1}) \oplus(\mathbf{1}, \mathbf{1}, \mathbf{1 0}) \oplus(\mathbf{1}, \mathbf{1}, \mathbf{5}) \oplus(\mathbf{2}, \mathbf{2}, \mathbf{5}) \oplus(\mathbf{2}, \mathbf{2}, \mathbf{1}) ; \\
& \mathbf{1 0}=(\mathbf{2}, \mathbf{2}, \mathbf{1}) \oplus(\mathbf{1}, \mathbf{1}, \mathbf{6})=(\mathbf{2}, \mathbf{2}, \mathbf{1}) \oplus(\mathbf{1}, \mathbf{1}, \mathbf{5}) \oplus(\mathbf{1}, \mathbf{1}, \mathbf{1}) ; \\
& 16=(\mathbf{2}, \mathbf{1}, \mathbf{4}) \oplus(\mathbf{1}, \mathbf{2}, \overline{\mathbf{4}})=(\mathbf{2}, \mathbf{1}, \mathbf{4}) \oplus(\mathbf{1}, \mathbf{2}, \mathbf{4}) ;
\end{aligned}
$$

II: $\mathfrak{d}_{5} \rightarrow \mathfrak{d}_{4} \oplus T_{1} \rightarrow \mathfrak{b}_{2} \oplus \mathfrak{a}_{1} \oplus T_{1}$;

$$
\begin{aligned}
\mathbf{4 5} & =\mathbf{2 8}_{0} \oplus \mathbf{1}_{0} \oplus \boldsymbol{8}_{v, 2} \oplus \boldsymbol{8}_{v,-2} \\
& =(\mathbf{1 0}, \mathbf{1})_{0} \oplus(\mathbf{1}, \mathbf{3})_{0} \oplus(\mathbf{5}, \mathbf{3})_{0} \oplus(\mathbf{1}, \mathbf{1})_{0} \oplus(\mathbf{5}, \mathbf{1})_{2} \oplus(\mathbf{1}, \mathbf{3})_{2} \oplus(\mathbf{5}, \mathbf{1})_{-2} \oplus(\mathbf{1}, \mathbf{3})_{-2} ;
\end{aligned}
$$

III: $\mathfrak{d}_{5} \rightarrow \mathfrak{b}_{4} \rightarrow \mathfrak{d}_{4} \rightarrow \mathfrak{a}_{1} \oplus \mathfrak{b}_{2}$

$$
\mathbf{4 5}=\mathbf{3 6} \oplus \mathbf{9}=\mathbf{2 8} \oplus \mathbf{8}_{v} \oplus \mathbf{8}_{v} \oplus \mathbf{1}=(\mathbf{3}, \mathbf{1}) \oplus(\mathbf{1}, \mathbf{1 0}) \oplus(\mathbf{3}, \mathbf{5}) \oplus(\mathbf{3}, \mathbf{1}) \oplus(\mathbf{1}, \mathbf{5}) \text {; }
$$

IV: $\mathfrak{d}_{5} \rightarrow \mathfrak{b}_{4} \rightarrow \mathfrak{a}_{1, I} \oplus \mathfrak{a}_{1, I I} \oplus \mathfrak{b}_{2}$ symmetric in $I, I I$

$$
\begin{aligned}
& \mathbf{4 5}=\mathbf{3 6} \oplus \mathbf{9}=(\mathbf{3}, \mathbf{1}, \mathbf{1}) \oplus(\mathbf{1}, \mathbf{3}, \mathbf{1}) \oplus(\mathbf{1}, \mathbf{1}, \mathbf{1 0}) \oplus(\mathbf{2}, \mathbf{2}, \mathbf{5}) \oplus(\mathbf{2}, \mathbf{2}, \mathbf{1}) \oplus(\mathbf{1}, \mathbf{1}, \mathbf{5}) \\
& \mathbf{1 0}=\mathbf{9} \oplus \mathbf{1}=(\mathbf{2}, \mathbf{2}, \mathbf{1}) \oplus(\mathbf{1}, \mathbf{1}, \mathbf{5}) \oplus(\mathbf{1}, \mathbf{1}, \mathbf{1}) ; \\
& \mathbf{1 6}=\mathbf{1 6}=(\mathbf{2}, \mathbf{1}, \mathbf{4}) \oplus(\mathbf{1}, \mathbf{2}, \mathbf{4})
\end{aligned}
$$

$\mathbf{V}: \mathfrak{d}_{5} \rightarrow \mathfrak{b}_{4} \rightarrow \mathfrak{a}_{1} \oplus \mathfrak{a}_{3} \rightarrow \mathfrak{a}_{1} \oplus \mathfrak{b}_{2}$

$$
\begin{aligned}
\mathbf{4 5} & =\mathbf{3 6} \oplus \mathbf{9}=(\mathbf{3}, \mathbf{1}) \oplus(\mathbf{1}, \mathbf{1 5}) \oplus(\mathbf{3}, \mathbf{6}) \oplus(\mathbf{3}, \mathbf{1}) \oplus(\mathbf{1}, \mathbf{6}) \\
& =(\mathbf{3}, \mathbf{1}) \oplus(\mathbf{1}, \mathbf{1 0}) \oplus(\mathbf{1}, \mathbf{5}) \oplus(\mathbf{3}, \mathbf{5}) \oplus(\mathbf{3}, \mathbf{1}) \oplus(\mathbf{3}, \mathbf{1}) \oplus(\mathbf{1}, \mathbf{5}) \oplus(\mathbf{1}, \mathbf{1}) ;
\end{aligned}
$$


VI: $\mathfrak{d}_{5} \rightarrow \mathfrak{b}_{3} \oplus \mathfrak{a}_{I} \rightarrow \mathfrak{a}_{3} \oplus \mathfrak{a}_{1} \rightarrow \mathfrak{b}_{2} \oplus \mathfrak{a}_{1}$

$$
\begin{aligned}
45 & =(\mathbf{2 1}, \mathbf{1}) \oplus(\mathbf{1}, \mathbf{3}) \oplus(\mathbf{7}, \mathbf{3})=(\mathbf{1 5}, \mathbf{1}) \oplus(\mathbf{6}, \mathbf{1}) \oplus(\mathbf{1}, \mathbf{3}) \oplus(\mathbf{6}, \mathbf{3}) \oplus(\mathbf{6}, \mathbf{1}) \\
& =(\mathbf{1 0}, \mathbf{1}) \oplus(\mathbf{5}, \mathbf{1}) \oplus(\mathbf{5}, \mathbf{1}) \oplus(\mathbf{1}, \mathbf{1}) \oplus(\mathbf{1}, \mathbf{3}) \oplus(\mathbf{5}, \mathbf{3}) \oplus(\mathbf{1}, \mathbf{3}) \oplus(\mathbf{5}, \mathbf{1}) \oplus(\mathbf{1}, \mathbf{1}) ;
\end{aligned}
$$

VII: $\mathfrak{d}_{5} \rightarrow \mathfrak{b}_{3} \oplus \mathfrak{a}_{I} \rightarrow \mathfrak{b}_{2} \oplus \mathfrak{a}_{1} \oplus T_{1}$

$45=(21,1) \oplus(\mathbf{1}, 3) \oplus(\mathbf{7}, 3)$

$$
=(\mathbf{1 0}, \mathbf{1})_{0} \oplus(\mathbf{1}, \mathbf{1})_{0} \oplus(\mathbf{5}, \mathbf{1})_{2} \oplus(\mathbf{5}, \mathbf{1})_{-2} \oplus(\mathbf{1}, \mathbf{3})_{0} \oplus(\mathbf{5}, \mathbf{3})_{0} \oplus(\mathbf{1}, \mathbf{3})_{2} \oplus(\mathbf{1}, \mathbf{3})_{-2} ;
$$

VIII: $\mathfrak{d}_{5} \rightarrow \underset{\substack{\text { symmetric in } I, I I \\ \text { sym }}}{\mathfrak{b}_{2, I} \oplus \mathfrak{b}_{2, I I}} \rightarrow \mathfrak{b}_{2, I} \oplus \mathfrak{a}_{1} \oplus \mathfrak{a}_{1}$

$$
\begin{aligned}
45= & (\mathbf{1 0}, \mathbf{1}) \oplus(\mathbf{1}, \mathbf{1 0}) \oplus(\mathbf{5}, \mathbf{5})=(\mathbf{1 0}, \mathbf{1}, \mathbf{1}) \oplus(\mathbf{1}, \mathbf{3}, \mathbf{1}) \oplus(\mathbf{1}, \mathbf{1}, \mathbf{3}) \oplus(\mathbf{1}, \mathbf{2}, \mathbf{2}) \\
& \oplus(\mathbf{5}, \mathbf{2}, \mathbf{2}) \oplus(\mathbf{5}, \mathbf{1}, \mathbf{1}) ; \\
\mathbf{1 0}= & (\mathbf{5}, \mathbf{1}) \oplus(\mathbf{1}, \mathbf{5})=(\mathbf{5}, \mathbf{1}, \mathbf{1}) \oplus(\mathbf{1}, \mathbf{2}, \mathbf{2}) \oplus(\mathbf{1}, \mathbf{1}, \mathbf{1}) ; \\
\mathbf{1 6}= & (\mathbf{4}, \mathbf{4})=(\mathbf{4}, \mathbf{2}, \mathbf{1}) \oplus(\mathbf{4}, \mathbf{1}, \mathbf{2}) ;
\end{aligned}
$$

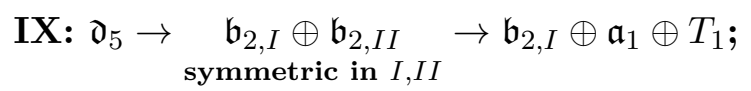

$$
\begin{aligned}
\mathbf{4 5} & =(\mathbf{1 0}, \mathbf{1}) \oplus(\mathbf{1}, \mathbf{1 0}) \oplus(\mathbf{5}, \mathbf{5}) \\
& =(\mathbf{1 0}, \mathbf{1})_{0} \oplus(\mathbf{1}, \mathbf{3})_{0} \oplus(\mathbf{1}, \mathbf{1})_{0} \oplus(\mathbf{1}, \mathbf{3})_{2} \oplus(\mathbf{1}, \mathbf{3})_{-2} \oplus(\mathbf{5}, \mathbf{3}) \oplus(\mathbf{5}, \mathbf{1})_{2} \oplus(\mathbf{5}, \mathbf{1})_{-2} ;
\end{aligned}
$$

$\mathbf{X}: \mathfrak{d}_{5} \rightarrow \underset{\text { symmetric in } I, I I}{\mathfrak{b}_{2, I} \oplus \mathfrak{b}_{2, I I}} \rightarrow \mathfrak{b}_{2, I} \oplus \mathfrak{a}_{1}$

$$
45=(10,1) \oplus(\mathbf{1}, \mathbf{1 0}) \oplus(\mathbf{5}, \mathbf{5})=(\mathbf{1 0}, \mathbf{1}) \oplus(\mathbf{1}, \mathbf{3}) \oplus(\mathbf{1}, \mathbf{7}) \oplus(\mathbf{5}, \mathbf{5}) .
$$

It thus follows that the $\operatorname{cases}^{36} I-X$ do not satisfy at least one of the aforementioned conditions i) and ii) of section 11.3 for $L(1,5)$ to be a submodel of $L(8,1)$. This proves that

$$
\begin{aligned}
& T_{3}(1,5,0) \not J_{3}^{\mathbb{Q}} ; \\
& (\mathrm{SO}(2,1) \otimes \mathrm{SO}(5) \otimes \mathrm{SO}(1,1))_{0} \ltimes(\mathbf{2}, \mathbf{5})_{3 / 2} \nsubseteq \mathrm{SO}(9,1) \otimes \mathrm{SO}(1,1) \subsetneq E_{6(-26)},
\end{aligned}
$$

and thus that the model $L(1,5)$ is not a submodel of $L(8,1)$, but rather only one of its descendants.

Open Access. This article is distributed under the terms of the Creative Commons Attribution License (CC-BY 4.0), which permits any use, distribution and reproduction in any medium, provided the original author(s) and source are credited.

\section{References}

[1] L. Andrianopoli et al., $N=2$ supergravity and $N=2$ super Yang-Mills theory on general scalar manifolds: Symplectic covariance, gaugings and the momentum map, J. Geom. Phys. 23 (1997) 111 [hep-th/9605032] [INSPIRE].

[2] L. Andrianopoli, R. D'Auria and S. Ferrara, Flat symplectic bundles of $N$ extended supergravities, central charges and black hole entropy, in APCTP Winter School on Dualities of Gauge and String Theories, (1997), pp. 283-323, DOI [hep-th/9707203] [INSPIRE].

\footnotetext{
${ }^{36}$ Some chains (such as $I$ and $I V$ ) of embeddings yield to the same $\mathfrak{b}_{2} \oplus \mathfrak{a}_{1}$ subalgebra of $\mathfrak{d}_{5}$.
} 
[3] L. Andrianopoli, R. D'Auria and S. Ferrara, U invariants, black hole entropy and fixed scalars, Phys. Lett. B 403 (1997) 12 [hep-th/9703156] [INSPIRE].

[4] L. Andrianopoli, R. D'Auria, S. Ferrara and M.A. Lledó, Gauging of flat groups in four-dimensional supergravity, JHEP 07 (2002) 010 [hep-th/0203206] [INSPIRE].

[5] L. Andrianopoli, R. D'Auria, S. Ferrara and M. Trigiante, Extremal black holes in supergravity, Lect. Notes Phys. 737 (2008) 661 [hep-th/0611345] [INSPIRE].

[6] L. Andrianopoli, R. D'Auria, S. Ferrara and M. Trigiante, Black-hole attractors in $N=1$ supergravity, JHEP 07 (2007) 019 [hep-th/0703178] [INSPIRE].

[7] A.A. Albert, A structure theory for Jordan algebras, Annals Math. Second Series 48 (1947) 546.

[8] S. Ferrara, R. Kallosh and A. Strominger, $N=2$ extremal black holes, Phys. Rev. D 52 (1995) R5412 [hep-th/9508072] [INSPIRE].

[9] A. Strominger, Macroscopic entropy of $N=2$ extremal black holes, Phys. Lett. B 383 (1996) 39 [hep-th/9602111] [INSPIRE].

[10] S. Ferrara and R. Kallosh, Supersymmetry and attractors, Phys. Rev. D 54 (1996) 1514 [hep-th/9602136] [INSPIRE].

[11] S. Ferrara and R. Kallosh, Universality of supersymmetric attractors, Phys. Rev. D 54 (1996) 1525 [hep-th/9603090] [INSPIRE].

[12] D.V. Alekseevsky, A. Marrani and A. Spiro, Special Vinberg cones and the entropy of BPS extremal black holes, JHEP 11 (2021) 100 [arXiv:2107.06797] [INSPIRE].

[13] J.C. Baez, The Octonions, Bull. Am. Math. Soc. 39 (2002) 145 [Erratum ibid. 42 (2005) 213] [math/0105155] [INSPIRE].

[14] L. Borsten, M.J. Duff, S. Ferrara, A. Marrani and W. Rubens, Small Orbits, Phys. Rev. D 85 (2012) 086002 [arXiv:1108.0424] [INSPIRE].

[15] L. Borsten, M.J. Duff and A. Marrani, Black Holes and Higher Composition Laws, arXiv: 2006.03574 [INSPIRE].

[16] S. Bellucci, S. Ferrara, M. Günaydin and A. Marrani, Charge orbits of symmetric special geometries and attractors, Int. J. Mod. Phys. A 21 (2006) 5043 [hep-th/0606209] [InSPIRE].

[17] S. Bellucci, S. Ferrara and A. Marrani, On some properties of the attractor equations, Phys. Lett. B 635 (2006) 172 [hep-th/0602161] [INSPIRE].

[18] S. Bellucci, S. Ferrara and A. Marrani, Supersymmetric mechanics. Vol. 2: The attractor mechanism and space time singularities, Lect. Notes Phys. 701 (2006).

[19] S. Bellucci, S. Ferrara, A. Shcherbakov and A. Yeranyan, Attractors and first order formalism in five dimensions revisited, Phys. Rev. D 83 (2011) 065003 [arXiv:1010.3516] [InSPIRE].

[20] P. Breitenlohner, D. Maison and G.W. Gibbons, Four-Dimensional Black Holes from Kaluza-Klein Theories, Commun. Math. Phys. 120 (1988) 295 [INSPIRE].

[21] R. Kallosh, T. Ortín and A.W. Peet, Entropy and action of dilaton black holes, Phys. Rev. D 47 (1993) 5400 [hep-th/9211015] [INSPIRE].

[22] R. Kallosh, Supersymmetric black holes, Phys. Lett. B 282 (1992) 80 [hep-th/9201029] [INSPIRE]. 
[23] J.M. Maldacena, Black holes in string theory, Ph.D. Thesis, Princeton University, U.S.A. (1996), hep-th/9607235 [INSPIRE].

[24] A.W. Peet, TASI lectures on black holes in string theory, in Theoretical Advanced Study Institute in Elementary Particle Physics (TASI 99): Strings, Branes, and Gravity, (2000), pp. 353-433, DOI [hep-th/0008241] [INSPIRE].

[25] B. Pioline, Lectures on black holes, topological strings and quantum attractors (2.0), Lect. Notes Phys. 755 (2008) 283 [INSPIRE].

[26] A. Dabholkar, Black hole entropy and attractors, Class. Quant. Grav. 23 (2006) S957 [INSPIRE].

[27] S. Ferrara, K. Hayakawa and A. Marrani, Lectures on Attractors and Black Holes, Fortsch. Phys. 56 (2008) 993 [arXiv:0805.2498] [INSPIRE].

[28] S. Bellucci, S. Ferrara, R. Kallosh and A. Marrani, Extremal Black Hole and Flux Vacua Attractors, Lect. Notes Phys. 755 (2008) 115 [arXiv:0711.4547] [INSPIRE].

[29] S. Bellucci, A. Marrani and R. Roychowdhury, On Quantum Special Kähler Geometry, Int. J. Mod. Phys. A 25 (2010) 1891 [arXiv:0910.4249] [InSPIRE].

[30] S. Bellucci, A. Marrani and R. Roychowdhury, Topics in Cubic Special Geometry, J. Math. Phys. 52 (2011) 082302 [arXiv: 1011.0705] [INSPIRE].

[31] L. Borsten, Aspects of M-Theory and Quantum Information. Ph.D. Thesis, Imperial College, U.K. (2010), https://spiral.imperial.ac.uk:8443/handle/10044/1/6051.

[32] E.B. Bogomolny, Stability of Classical Solutions, Sov. J. Nucl. Phys. 24 (1976) 449 [Yad. Fiz. 24 (1976) 861] [INSPIRE].

[33] M.K. Prasad and C.M. Sommerfield, Exact Classical Solution for the 't Hooft Monopole and the Julia-Zee Dyon, Phys. Rev. Lett. 35 (12) 760 (1975).

[34] A. Ceresole, R. D'Auria and S. Ferrara, The symplectic structure of $N=2$ supergravity and its central extension, Nucl. Phys. B Proc. Suppl. 46 (1996) 67 [hep-th/9509160] [InSPIRE].

[35] G. Lopes Cardoso, B. de Wit and T. Mohaupt, Corrections to macroscopic supersymmetric black hole entropy, Phys. Lett. B 451 (1999) 309 [hep-th/9812082] [INSPIRE].

[36] G. Lopes Cardoso, B. de Wit and T. Mohaupt, Macroscopic entropy formulae and nonholomorphic corrections for supersymmetric black holes, Nucl. Phys. B 567 (2000) 87 [hep-th/9906094] [INSPIRE].

[37] S. Cecotti, Homogeneous Kähler Manifolds and T Algebras in $N=2$ Supergravity and Superstrings, Commun. Math. Phys. 124 (1989) 23 [INSPIRE].

[38] A. Ceresole, S. Ferrara, A. Gnecchi and A. Marrani, d-Geometries Revisited, JHEP 02 (2013) 059 [arXiv: 1210.5983] [INSPIRE].

[39] A. Ceresole, S. Ferrara and A. Marrani, 4d/5d Correspondence for the Black Hole Potential and its Critical Points, Class. Quant. Grav. 24 (2007) 5651 [arXiv:0707.0964] [INSPIRE].

[40] B.L. Cerchiai and B. van Geemen, From qubits to E7, J. Math. Phys. 51 (2010) 122203 [arXiv: 1003.4255] [INSPIRE].

[41] E. Cremmer and B. Julia, The $N=8$ Supergravity Theory. 1. The Lagrangian, Phys. Lett. B 80 (1978) 48 [INSPIRE].

[42] E. Cremmer and B. Julia, The SO(8) Supergravity, Nucl. Phys. B 159 (1979) 141 [inSPIRE]. 
[43] V. Cortés, Homogeneous special geometry, Transform. Groups 1 (1996) 337.

[44] M. Cvetič and C.M. Hull, Black holes and U duality, Nucl. Phys. B 480 (1996) 296 [hep-th/9606193] [INSPIRE].

[45] M. Cvetič and D. Youm, All the static spherically symmetric black holes of heterotic string on a six torus, Nucl. Phys. B 472 (1996) 249 [hep-th/9512127] [INSPIRE].

[46] M. Cvetič and A.A. Tseytlin, Solitonic strings and BPS saturated dyonic black holes, Phys. Rev. D 53 (1996) 5619 [Erratum ibid. 55 (1997) 3907] [hep-th/9512031] [INSPIRE].

[47] E. Cremmer and A. Van Proeyen, Classification of Kähler Manifolds in $N=2$ Vector Multiplet Supergravity Couplings, Class. Quant. Grav. 2 (1985) 445 [INSPIRE].

[48] M.J. Duff, String triality, black hole entropy and Cayley's hyperdeterminant, Phys. Rev. D 76 (2007) 025017 [hep-th/0601134] [INSPIRE].

[49] L. Borsten, M.J. Duff, A. Marrani and W. Rubens, On the Black-Hole/Qubit Correspondence, Eur. Phys. J. Plus 126 (2011) 37 [arXiv:1101.3559] [InSPIRE].

[50] L. Borsten, M.J. Duff and P. Levay, The black-hole/qubit correspondence: an up-to-date review, Class. Quant. Grav. 29 (2012) 224008 [arXiv:1206.3166] [InSPIRE].

[51] R. D'Auria, S. Ferrara and M. Trigiante, Critical points of the Black-Hole potential for homogeneous special geometries, JHEP 03 (2007) 097 [hep-th/0701090] [INSPIRE].

[52] K. Dasgupta, V. Hussin and A. Wissanji, Quaternionic Kähler Manifolds, Constrained Instantons and the Magic Square. I, Nucl. Phys. B 793 (2008) 34 [arXiv:0708.1023] [INSPIRE].

[53] B. de Wit and A. Van Proeyen, Special geometry, cubic polynomials and homogeneous quaternionic spaces, Commun. Math. Phys. 149 (1992) 307 [hep-th/9112027] [INSPIRE].

[54] B. de Wit and A. Van Proeyen, Broken sigma model isometries in very special geometry, Phys. Lett. B 293 (1992) 94 [hep-th/9207091] [INSPIRE].

[55] B. de Wit and A. Van Proeyen, Isometries of special manifolds, (1995) [hep-th/9505097] [INSPIRE].

[56] B. de Wit, F. Vanderseypen and A. Van Proeyen, Symmetry structure of special geometries, Nucl. Phys. B 400 (1993) 463 [hep-th/9210068] [inSPIRE].

[57] A. Sen, Extremal black holes and elementary string states, Mod. Phys. Lett. A 10 (1995) 2081 [hep-th/9504147] [INSPIRE].

[58] G.T. Horowitz and A. Strominger, Counting states of near extremal black holes, Phys. Rev. Lett. 77 (1996) 2368 [hep-th/9602051] [INSPIRE].

[59] S. Ferrara and M. Günaydin, Orbits of exceptional groups, duality and BPS states in string theory, Int. J. Mod. Phys. A 13 (1998) 2075 [hep-th/9708025] [INSPIRE].

[60] S. Ferrara and M. Günaydin, Orbits and Attractors for $N=2$ Maxwell-Einstein Supergravity Theories in Five Dimensions, Nucl. Phys. B 759 (2006) 1 [hep-th/0606108] [INSPIRE].

[61] S. Ferrara, G.W. Gibbons and R. Kallosh, Black holes and critical points in moduli space, Nucl. Phys. B 500 (1997) 75 [hep-th/9702103] [inSPIRE].

[62] S. Ferrara, E.G. Gimon and R. Kallosh, Magic supergravities, $N=8$ and black hole composites, Phys. Rev. D 74 (2006) 125018 [hep-th/0606211] [INSPIRE]. 
[63] S. Ferrara and A. Marrani, On the Moduli Space of non-BPS Attractors for $N=2$ Symmetric Manifolds, Phys. Lett. B 652 (2007) 111 [arXiv:0706.1667] [InSPIRE].

[64] D.S. Freed, Special Kähler manifolds, Commun. Math. Phys. 203 (1999) 31 [hep-th/9712042] [INSPIRE].

[65] A. Gnecchi and N. Halmagyi, Supersymmetric black holes in $A d S_{4}$ from very special geometry, JHEP 04 (2014) 173 [arXiv:1312.2766] [InSPIRE].

[66] M. Günaydin, K. Koepsell and H. Nicolai, Conformal and quasiconformal realizations of exceptional Lie groups, Commun. Math. Phys. 221 (2001) 57 [hep-th/0008063] [inSPIRE].

[67] M. Günaydin, S. Kachru and A. Tripathy, Black holes and Bhargava's invariant theory, J. Phys. A 53 (2020) 444001 [arXiv: 1903.02323] [InSPIRE].

[68] M. Gasperini, J. Maharana and G. Veneziano, From trivial to nontrivial conformal string backgrounds via $O(d, d)$ transformations, Phys. Lett. B 272 (1991) 277 [INSPIRE].

[69] J. Maharana and J.H. Schwarz, Noncompact symmetries in string theory, Nucl. Phys. B 390 (1993) 3 [hep-th/9207016] [INSPIRE].

[70] M. Günaydin and O. Pavlyk, Generalized spacetimes defined by cubic forms and the minimal unitary realizations of their quasiconformal groups, JHEP 08 (2005) 101 [hep-th/0506010] [INSPIRE].

[71] M. Günaydin and O. Pavlyk, Spectrum Generating Conformal and Quasiconformal U-duality Groups, Supergravity and Spherical Vectors, JHEP 04 (2010) 070 [arXiv:0901.1646] [INSPIRE].

[72] M. Günaydin and O. Pavlyk, Quasiconformal Realizations of $E_{6(6)}, E_{7(7)}, E_{8(8)}$ and $\mathrm{SO}(n+3, m+3), N \geqslant 4$ Supergravity and Spherical Vectors [inSPIRE].

[73] B. De Witt and C. De Witt, Black Holes, Gordon and Breach, New York, U.S.A. (1973).

[74] S.W. Hawking and W. Israel, General Relativity, Cambridge University Press, U.K. (1979).

[75] R.M. Wald, General Relativity, University of Chicago Press, U.S.A. (1984).

[76] M. Günaydin, G. Sierra and P.K. Townsend, Exceptional Supergravity Theories and the MAGIC Square, Phys. Lett. B 133 (1983) 72 [INSPIRE].

[77] M. Günaydin, G. Sierra and P.K. Townsend, The Geometry of $N=2$ Maxwell-Einstein Supergravity and Jordan Algebras, Nucl. Phys. B 242 (1984) 244 [InSPIRE].

[78] M. Günaydin, G. Sierra and P.K. Townsend, More on $d=5$ Maxwell-Einstein Supergravity: Symmetric Spaces and Kinks, Class. Quant. Grav. 3 (1986) 763 [INSPIRE].

[79] N. Halmagyi, BPS Black Hole Horizons in $N=2$ Gauged Supergravity, JHEP 02 (2014) 051 [arXiv:1308.1439] [INSPIRE].

[80] K. Hasebe, The Split-Algebras and Non-compact Hopf Maps, J. Math. Phys. 51 (2010) 053524 [arXiv: 0905. 2792] [INSPIRE].

[81] S.W. Hawking, Black hole explosions, Nature 248 (1974) 30 [InSPIRE].

[82] S.W. Hawking, Gravitational radiation from colliding black holes, Phys. Rev. Lett. 26 (1971) 1344 [INSPIRE].

[83] J.D. Bekenstein, Black holes and entropy, Phys. Rev. D 7 (1973) 2333 [inSPIRE]. 
[84] S.W. Hawking and R. Penrose, The singularities of gravitational collapse and cosmology, Proc. Roy. Soc. Lond. A 314 (1970) 529 [inSPIRE].

[85] C.M. Hull and P.K. Townsend, Unity of superstring dualities, Nucl. Phys. B 438 (1995) 109 [hep-th/9410167] [INSPIRE].

[86] P. Jordan, J. v. Neumann and E. Wigner, On an Algebraic Generalization of the Quantum Mechanical Formalism, Annals Math. Second Series 35 (1934) 29.

[87] V.G. Kac, Some Remarks on Nilpotent Orbits, J. Algebra 64 (1980) 190.

[88] R. Kallosh, A.D. Linde, T. Ortín, A.W. Peet and A. Van Proeyen, Supersymmetry as a cosmic censor, Phys. Rev. D 46 (1992) 5278 [hep-th/9205027] [INSPIRE].

[89] S. Krutelevich, Jordan Algebras, Exceptional Groups, and Bhargava Composition, J. Algebra 314 (2007) 924 [math/0411104].

[90] Y. Kim and F-O. Schreyer, An explicit matrix factorization of cubic hypersurfaces of small dimension, [arXiv:1905.09626].

[91] C.I. Lazaroiu and C.S. Shahbazi, Four-dimensional geometric supergravity and electromagnetic duality: a brief guide for mathematicians, arXiv:2006.16157 [INSPIRE].

[92] A. Strominger and C. Vafa, Microscopic origin of the Bekenstein-Hawking entropy, Phys. Lett. B 379 (1996) 99 [hep-th/9601029] [INSPIRE].

[93] C.G. Callan and J.M. Maldacena, D-brane approach to black hole quantum mechanics, Nucl. Phys. B 472 (1996) 591 [hep-th/9602043] [INSPIRE].

[94] R. Dijkgraaf, E.P. Verlinde and H.L. Verlinde, BPS spectrum of the five-brane and black hole entropy, Nucl. Phys. B 486 (1997) 77 [hep-th/9603126] [INSPIRE].

[95] D.M. Kaplan, D.A. Lowe, J.M. Maldacena and A. Strominger, Microscopic entropy of $N=2$ extremal black holes, Phys. Rev. D 55 (1997) 4898 [hep-th/9609204] [INSPIRE].

[96] J.M. Maldacena, $N=2$ extremal black holes and intersecting branes, Phys. Lett. $B 403$ (1997) 20 [hep-th/9611163] [INSPIRE].

[97] J.M. Maldacena, A. Strominger and E. Witten, Black hole entropy in M-theory, JHEP 12 (1997) 002 [hep-th/9711053] [INSPIRE].

[98] A. Marrani, P.K. Tripathy and T. Mandal, Supersymmetric Black Holes and Freudenthal Duality, Int. J. Mod. Phys. A 32 (2017) 1750114 [arXiv:1703.08669] [inSPIRE].

[99] G.W. Moore, Arithmetic and attractors, hep-th/9807087 [INSPIRE].

[100] G.W. Moore, Strings and Arithmetic, in Les Houches School of Physics: Frontiers in Number Theory, Physics and Geometry, (2007), pp. 303-359, DOI [hep-th/0401049] [INSPIRE].

[101] A. Marrani, G. Pradisi, F. Riccioni and L. Romano, Nonsupersymmetric magic theories and Ehlers truncations, Int. J. Mod. Phys. A 32 (2017) 1750120 [arXiv:1701.03031] [InSPIRE].

[102] A. Marrani and L. Romano, Orbits in nonsupersymmetric magic theories, Int. J. Mod. Phys. A 34 (2019) 1950190 [arXiv:1906.05830] [INSPIRE].

[103] T. Mandal and P.K. Tripathy, On the Uniqueness of Supersymmetric Attractors, Phys. Lett. $B 749$ (2015) 221 [arXiv:1506. 06276] [INSPIRE].

[104] A. Marrani, P. Truini and M. Rios, The Magic of Being Exceptional, J. Phys. Conf. Ser. 1194 (2019) 012075 [arXiv: 1811.11208] [InSPIRE]. 
[105] K. Goldstein, N. Iizuka, R.P. Jena and S.P. Trivedi, Non-supersymmetric attractors, Phys. Rev. D 72 (2005) 124021 [hep-th/0507096] [INSPIRE].

[106] R. Kallosh, New attractors, JHEP 12 (2005) 022 [hep-th/0510024] [INSPIRE].

[107] P.K. Tripathy and S.P. Trivedi, Non-supersymmetric attractors in string theory, JHEP 03 (2006) 022 [hep-th/0511117] [INSPIRE].

[108] R. Kallosh, N. Sivanandam and M. Soroush, The Non-BPS black hole attractor equation, JHEP 03 (2006) 060 [hep-th/0602005] [INSPIRE].

[109] A. Dabholkar, A. Sen and S.P. Trivedi, Black hole microstates and attractor without supersymmetry, JHEP 01 (2007) 096 [hep-th/0611143] [INSPIRE].

[110] M. Noether, Zur Theorie der Thetafunctionen van beliebig vielen Argumenten, Math. Ann. 16 (1880) 270.

[111] R. Kallosh and T. Ortín, Charge quantization of axion-dilaton black holes, Phys. Rev. D 48 (1993) 742 [hep-th/9302109] [INSPIRE].

[112] E. Bergshoeff, R. Kallosh and T. Ortín, Stationary axion/dilaton solutions and supersymmetry, Nucl. Phys. B 478 (1996) 156 [hep-th/9605059] [INSPIRE].

[113] R.R. Khuri and T. Ortín, A nonsupersymmetric dyonic extreme Reissner-Nordström black hole, Phys. Lett. B 373 (1996) 56 [hep-th/9512178] [INSPIRE].

[114] T. Ortín, Extremality versus supersymmetry in stringy black holes, Phys. Lett. B 422 (1998) 93 [hep-th/9612142] [INSPIRE].

[115] L. Pirio and F. Russo, Extremal varieties 3-rationally connected by cubics, quadro-quadric Cremona transformations and rank 3 Jordan algebras, J.Reine Angew.Math. 716 (2016) 229 [arXiv:1109.3573].

[116] L. Pirio and F. Russo, Quadro-quadric cremona transformations in low dimensions via the JC-correspondence, Annales Inst. Fourier 64 (2014) 71 [arXiv:1204.0428].

[117] F. Russo, On the geometry of some special projective varieties, in Lecture Notes of the UMI 18, Springer (2016).

[118] J.H. Schwarz and A. Sen, Duality symmetries of 4-D heterotic strings, Phys. Lett. B 312 (1993) 105 [hep-th/9305185] [INSPIRE].

[119] J.H. Schwarz and A. Sen, Duality symmetric actions, Nucl. Phys. B 411 (1994) 35 [hep-th/9304154] [INSPIRE].

[120] A. Sen, Black hole solutions in heterotic string theory on a torus, Nucl. Phys. B 440 (1995) 421 [hep-th/9411187] [INSPIRE].

[121] A. Sen, Quantization of dyon charge and electric magnetic duality in string theory, Phys. Lett. B 303 (1993) 22 [hep-th/9209016] [INSPIRE].

[122] M. Shmakova, Calabi-Yau black holes, Phys. Rev. D 56 (1997) 540 [hep-th/9612076] [INSPIRE].

[123] S. Ferrara and A. Strominger, $\mathcal{N}=2$ space-time supersymmetry and Calabi Yau Moduli Space, in: Proceedings of College Station Workshop "Strings '89", pag. 245, eds. Arnowitt et al., World Scientific (1989).

[124] P. Candelas and X. de la Ossa, Moduli Space of Calabi-Yau Manifolds, Nucl. Phys. B 355 (1991) 455 [INSPIRE]. 
[125] B. de Wit and A. Van Proeyen, Potentials and Symmetries of General Gauged $N=2$ Supergravity: Yang-Mills Models, Nucl. Phys. B 245 (1984) 89 [INSPIRE].

[126] E. Cremmer et al., Vector Multiplets Coupled to $N=2$ Supergravity: SuperHiggs Effect, Flat Potentials and Geometric Structure, Nucl. Phys. B 250 (1985) 385 [InSPIRE].

[127] B. de Wit, P.G. Lauwers and A. Van Proeyen, Lagrangians of $N=2$ Supergravity-Matter Systems, Nucl. Phys. B 255 (1985) 569 [INSPIRE].

[128] L. Castellani, R. D'Auria and S. Ferrara, Special geometry without special coordinates, Class. Quant. Grav. 7 (1990) 1767 [INSPIRE].

[129] L. Castellani, R. D'Auria and S. Ferrara, Special Kähler Geometry: An Intrinsic Formulation From $N=2$ Space-time Supersymmetry, Phys. Lett. B 241 (1990) 57 [InSPIRE].

[130] J.H. Schwarz, Lectures on superstring and M-theory dualities: Given at ICTP Spring School and at TASI Summer School, Nucl. Phys. B Proc. Suppl. 55 (1997) 1 [hep-th/9607201] [INSPIRE].

[131] M.J. Duff, M theory (The Theory formerly known as strings), Int. J. Mod. Phys. A 11 (1996) 5623 [hep-th/9608117] [INSPIRE].

[132] A. Sen, Unification of string dualities, Nucl. Phys. B Proc. Suppl. 58 (1997) 5 [hep-th/9609176] [INSPIRE].

[133] A. Strominger, Special Geometry, Commun. Math. Phys. 133 (1990) 163 [InSPIRE].

[134] B. van Geemen, Schottky-Jung relations and vector bundles on hyperelliptic curves, Math. Ann. 281 (1988) 431.

[135] E.B. Vinberg, The Theory of Homogeneous Convex Cones, in Transactions of the Moscow Math. Society for the year 1963, p. 340, Providence, RI, U.S.A., American Mathematical Society, (1965).

[136] J.H. Schwarz, M theory extensions of T duality, in Frontiers in Quantum Field Theory in Honor of the 60th Birthday of Prof. K. Kikkawa, (1995), pp. 3-14 hep-th/9601077 [INSPIRE].

[137] C. Vafa, Evidence for F-theory, Nucl. Phys. B 469 (1996) 403 [hep-th/9602022] [INSPIRE].

[138] E. Witten, String theory dynamics in various dimensions, Nucl. Phys. B 443 (1995) 85 [hep-th/9503124] [INSPIRE].

[139] R.M. Wald, Black hole entropy is the Noether charge, Phys. Rev. D 48 (1993) R3427 [gr-qc/9307038] [INSPIRE]. 